\title{
Analysis of Multiuser Cellular Systems Over Heterogeneous Channels
}

by

\author{
Harsh Tataria
}

A thesis

submitted to the Victoria University of Wellington

in fulfilment of the

requirements for the degree of

Doctor of Philosophy

in Electronic (Communications) Engineering.

Victoria University of Wellington, New Zealand 



\begin{abstract}
This thesis considers the analysis of current and future cellular communication systems. The main focus is on multiuser multiple-input multiple-output (MUMIMO) antenna systems. The goal of this work is to characterize the achievable spectral efficiency of MU-MIMO systems, as well as to analyze the performance of practical, linear MU-MIMO transceiver structures in heterogeneous propagation environments. The analytical derivations in this thesis are based on the mathematical theory of finite and large dimensional random matrices. A collection of new general random matrix theory results, which permit efficient numerical evaluation are derived.

With downlink regularized zero-forcing (RZF) processing at a cellular base station (BS), a general framework for the analysis of the expected (average) signal-to-interference-plus-noise-ratio (SINR) and ergodic sum spectral efficiency is developed for uncorrelated and semi-correlated Rayleigh fading, as well as uncorrelated Ricean fading propagation channels. In contrast to existing results, the presented analyses are extremely general, applicable to single-cellular, multicellular, as well as distributed antenna systems. These systems could consist of arbitrary numbers of transmit and receive antennas, link signal-to-noise-ratios (SNRs), equal and unequal transmit correlation structures, and line-of-sight (LoS) levels, respectively. Numerical results are presented for single-cellular, as well as for two-tier multi-cellular systems demonstrating the impact of novel BS coordination strategies to suppress dominant inter-cellular interference.

With dominant LoS directions in the propagation channel, the instantaneous downlink zero-forcing (ZF) SNR of a given terminal is analyzed. The ZF SNR is shown to be approximated by a gamma distribution for any number of transmit and receive antennas, link SNRs, and LoS levels. Furthermore, for moderately sized MU-MIMO systems, simplified instantaneous and ergodic
\end{abstract}


sum spectral efficiency analyses are presented with RZF, ZF and matched-filter (MF) transmission on the downlink, and minimum-mean-squared-error, ZF and maximum-ratio combining (MRC) on the uplink, respectively. The simple nature of the derived expressions lead to the discovery of several valuable system level insights as a function of the contributing network parameters. Numerical results are presented for conventional and moderate MU-MIMO systems.

Considering downlink semi-correlated Rayleigh fading channels with spatial correlation at the $\mathrm{BS}$, it is mathematically proven that common correlation patterns for each terminal predicts lower ergodic sum spectral efficiencies in comparison to terminal specific correlation patterns. Closed-form approximations for the expected SINR and ergodic sum spectral efficiency are derived for both MF and ZF precoding, demonstrating the sensitivity of unequal correlation structures on the expected signal, interference and noise powers, respectively. The presented numerical results provide a cautionary tale of the impact of unequal correlation patterns on MU-MIMO performance and the importance of modeling this phenomenon.

Finally, an approximate uplink performance analysis of large MU-MIMO systems with MRC and space-constrained uniform linear antenna arrays (ULA) is presented for semi-correlated Ricean fading channels. A space-constrained channel model is proposed, encapsulating the effects of unequal receive spatial correlation, unequal LoS levels, and unequal link gains for each terminal. The per-terminal and cell-wide ergodic sum spectral efficiencies are characterized and numerous practical special cases are presented. A limiting analysis of the ergodic per-terminal and cell-wide spectral efficiencies is also carried out, as the number of BS antennas grow without bound with a finite number of terminals and fixed physical dimensions of the ULA. Numerical results demonstrate the impact of space-constrained ULAs on the MU-MIMO system performance with variation in the LoS levels, correlation structures, physical array dimensions, and system size, respectively. 


\section{Statement of Originality}

The research presented in the thesis has not been submitted for any other degree or reward in any other university or educational institution.

The work presented in this thesis is the result of original research carried out by myself, in collaboration with my advisors, Prof. Peter J. Smith (Professor of Statistics, Victoria University of Wellington, New Zealand) and Dr. Pawel A. Dmochowski (Senior Lecturer of Electronic Engineering, Victoria University of Wellington, New Zealand).

This work was carried out with the following external collaborators: Prof. Mansoor Shafi (Research Fellow, Spark New Zealand, Wellington, New Zealand), Prof. Larry J. Greenstein (Emeritus Research Professor of Electrical Engineering, Rutgers University, North Brunswick, NJ, USA), Prof. Andreas F. Molisch (Professor of Electrical Engineering, University of Southern California, Los Angeles, CA, USA), and Dr. Michail Matthaiou (Senior Lecturer of Electronic Engineering, Queen's University Belfast, Belfast, Northern Ireland, UK).

The substantial majority of this work is my own.

Harsh Tataria

School of Engineering and Computer Science

Victoria University of Wellington, Wellington, New Zealand

March 31, 2017 


\section{Acknowledgments}

First of all, I am grateful to God for giving me the opportunity to explore his world. This thesis aims to point out some of the beauties of nature, which he constantly inspired me to explore.

During this journey, my work has benefited from many individuals for whom I would like to take this opportunity to express my heartiest gratitude. In particular, I am extremely grateful to Prof. Mansoor Shafi for believing in me, and for giving me the opportunity to pursue graduate studies. If it was not for Prof. Shafi, I would not have had the chance to make this contribution. August 20, 2013 will forever be ingrained in my memory as the day Prof. Shafi decided to invite me, after an ECEN 410: Advanced Communications Engineering lecture, to discuss my prospects as a doctoral candidate. I consider this to be the turning point of my career. Right from my undergraduate studies, I have received invaluable support and world class technical guidance from Prof. Shafi. I have grately benefited from his exceptional vision and mentoring. The contribution of this thesis would not have been the same without him.

I also consider myself very fortunate to be one of the students of Prof. Peter J. Smith. His wisdom, technical insights, sharp focus, on-going encouragement, and complete belief in my ability was a constant source of motivation for me. This has resulted in research outputs which I would have previously considered to be impossible. Prof. Smith's unique skill to bring together communications and random matrix theory to tackle the most difficult cellular engineering problems will always serve as an inspiration to me. I am truly privileged to have him as a mentor. I would also like to thank Dr. Pawel A. Dmochowski, for his support throughout the doctoral candidature. Dr. Dmochowski, Prof. Smith, 
and Prof. Shafi provided critical feedback, creative research ideas, and were invaluable in the progression of research.

I would like to convey a special thank you to Prof. Larry J. Greenstein and Prof. Andreas F. Molisch, who have contributed significantly to the material presented in this thesis. They have provided world leading technical guidance, and additional mentoring. It was an absolute pleasure and a privilage to be mentored by these great minds of cellular communications, without whom the thesis would not be as it is. Furthermore, I would like to thank Dr. Michail Matthaiou for his technical and non-technical guidance throughout the thesis. I have learnt a great deal from Dr. Matthaiou. At this point, I would like to make a special mention to Prof. Dale A. Carnegie for his help and encouragement throughout the course of the thesis. I will always remeber the lessons Prof. Carnegie taught me. He has had a deep impact on my outlook as a person.

I would like to thank my various sources of financial support. Firstly, I would like to acknowledge the New Zealand Government for their help, allowing me to complete the Ph.D. degree. I am also grateful for the support of Mrs. Liz Richardson and Te Ropu Awhina at Victoria University of Wellington for their generosity and a wealth of support. Moreover, I consider myself lucky to have been the receipient of the Shafi Family Scholarship, which provided valuable financial support at critical stages of my Ph.D candidature.

A special mention must be given to a dear colleague and my good friend, Dr. Callum T. Neil, for providing constant encouragement and for many useful technical discussions during the course of the Ph.D. Other colleagues whom I would like to thank include Dr. Sudhir Singh, Dr. Jawad Mirza, Dr. Ramoni O. Adeogun, and Dr. Fatih R. Ustok.

I would also like to take this opportunity to thank my friends: Mr. Angus Cameron, Mr. Lewis Richards, Mr. Esekia Faiga, Mr. Arama Tairea, Mr. Stewart J. Rhodes, Mr. Lance Tollenaar, Mr. Jared L. Crouch, and Mr. Ravi M. Maisuria for their help in proof reading parts of the thesis, and their continuous support.

Finally, I can never forget the unconditional love of my family. Without 
them, nothing would have been possible. I would like to thank my parents, Mr. Paresh and Mrs. Anita Tataria, whom I have witnessed strugling and sacrificing for me, time and time again. It was them who implanted the value of education in my heart. I would also like to thank my sister, Ms. Akruti Tataria, and my grandparents, Mr. Anil and Mrs. Kamla Tataria for their unwaivering love and support.

Most importantly, this thesis is dedicated to my wife, Mrs. Chandni Tataria, whom I have had the pleasure of sharing my life with thus far. Thank you Chandni, for the love, patience, encouragement, and sacrifice. I am grately indebted to you for your support. I would not be who I am without you. 


\section{Contents}

List of Symbols and Operators $\quad$ Xv

1 Introduction 1

1.1 Motivation Statement . . . . . . . . . . . . . . 1

1.2 Problem Statement and Focus of the Thesis . . . . . . . . 5

1.3 Main Contributions of the Thesis . . . . . . . . . . . 6

1.3.1 Coordinated RZF Precoding in Multi-Cellular Systems . . . . . . . . . . . . . . . . . 6

1.3.2 RZF Precoding in Ricean Fading . . . . . . . . . 7

1.3.3 Simplified Analysis With Linear Transceivers . . . . . . . 8

1.3.4 Linear Precoding Analysis With Unequal Spatial Correlation ......................... 9

1.3.5 Uplink MRC Analysis With Fixed Array Sizes . . . . . . 10

1.4 Thesis Outline . . . . . . . . . . . . . . . 11

1.5 Publications . . . . . . . . . . . . . . . 14

2 Statistical Channel Models and Random Matrix Theory 17

2.1 Wireless Channels and Models . . . . . . . . . . . . 18

2.1.1 A Typical Multipath Environment . . . . . . . . 18

2.1 .2 Large-Scale Fading . . . . . . . . . . . . . . 20

2.1.3 Small-Scale Fading . . . . . . . . . . . . . . . 22

2.2 SISO Channels . . . . . . . . . . . . . . . . . 23

2.3 MIMO-OFDM Channels . . . . . . . . . . . . . . . . . 25

2.3.1 Uncorrelated I.I.D. Rayleigh Fading . . . . . . . . . . . 28 
2.3.2 Semi-Correlated Rayleigh Fading . . . . . . . . . . . 29

2.3.3 Uncorrelated Ricean Fading . . . . . . . . . . . . . 32

2.3.4 Ergodic Spectral Efficiency . . . . . . . . . . . . 34

2.4 Eigenvalue Densities of Wishart Matrices . . . . . . . . . 36

2.4.1 Uncorrelated Central Wishart Matrices . . . . . . . . . 36

2.4.2 Correlated Central Wishart Matrices . . . . . . . . 37

2.4.3 Uncorrelated Non-Central Wishart Matrices . . . . . . . 43

3 Multiuser MIMO Systems $\quad 49$

3.1 System Operation and Assumptions . . . . . . . . . . . . 50

3.2 Heterogeneity in MU-MIMO Systems . . . . . . . . . . . . . . . 54

3.3 Signal Models . . . . . . . . . . . . . . . 56

3.3.1 Uplink Transmission . . . . . . . . . . . . . . 57

3.3.2 Downlink Transmission . . . . . . . . . . . . 58

3.4 Multiuser Signal Processing Techniques . . . . . . . . . . . . 59

3.4.1 Uplink Linear Combining . . . . . . . . . . . 60

3.4.2 Downlink Linear Precoding . . . . . . . . . . . 65

3.4.3 Expected Per-Terminal SINR Approximation . . . . . . . 69

3.5 Large Multiuser MIMO Systems . . . . . . . . . . . . . . 70

3.5.1 Convergence to Favorable Propagation Conditions . . . . 71

3.5.2 Impact on Sum Spectral Efficiency . . . . . . . . . . 72

3.5.3 Space-Constrained MU-MIMO Systems . . . . . . . . . 73

4 Coordinated RZF Precoding in Multi-Cellular Systems $\quad 75$

4.1 Introduction . . . . . . . . . . . . . 76

4.2 System Model . . . . . . . . . . . . . . . . . . . . . 80

4.2.1 Downlink Received Signal . . . . . . . . . . 80

4.2 .2 RZF Precoding . . . . . . . . . . . . . . . . . 82

4.2.3 Base Station Coordination Strategies . . . . . . . . . 84

4.3 Analysis Methodology . . . . . . . . . . . . . . . 86

4.3.1 Integrals and Special Functions . . . . . . . . . 86 
4.3.2 Expected SINR and Ergodic Sum Spectral Efficiency Analysis ....................... 87

4.4 High SNR ZF Approximation . . . . . . . . . . . . . . . 101

4.5 Numerical Results and Discussion . . . . . . . . . . . . . . . 104

4.5.1 Simulation Settings . . . . . . . . . . . . . 105

4.6 Concluding Remarks . . . . . . . . . . . . . . . . 116

$\begin{array}{lll}5 & \text { RZF Analysis in Ricean Fading } & 117\end{array}$

5.1 Introduction . . . . . . . . . . . . . . . . . . 118

5.2 System Model . . . . . . . . . . . . . . . . . . . . . 121

5.2 .1 Channel Model . . . . . . . . . . . . . . . . . . 121

5.2.2 RZF SINR and Ergodic Sum Spectral Efficiency . . . . . 123

5.3 Expected SINR and Ergodic Sum Spectral Efficiency Approxima-

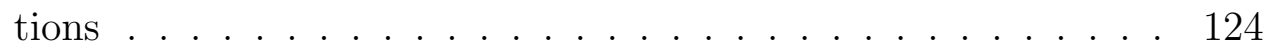

5.3.1 Computation of Expected Signal Power . . . . . . . . 125

5.3.2 Computation of Expected Interference Power . . . . . . . 130

5.4 Approximation to the RZF SINR . . . . . . . . . . . . . 131

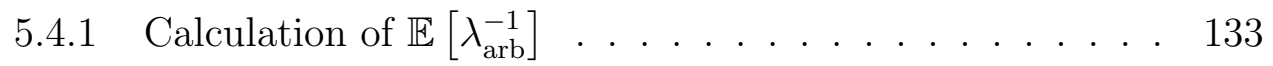

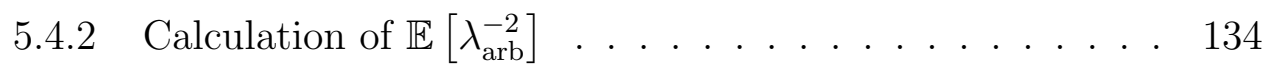

5.4 .3 Calculation of $\mathbb{E}\left[\lambda_{1}^{-1} \lambda_{2}^{-1}\right] \ldots \ldots \ldots \ldots . \ldots . \ldots . \ldots 134$

5.5 Numerical Results and Discussion . . . . . . . . . . . . 136

5.6 Concluding Remarks . . . . . . . . . . . . . . . . . . 142

6 Simplified Performance Analysis With Linear Transceivers 145

6.1 Introduction . . . . . . . . . . . . . . . . . . 146

6.2 Downlink and Uplink System Models . . . . . . . . . . . . . . . 149

6.2.1 Downlink Linear Precoding . . . . . . . . . . . . . 150

6.2.2 Uplink Linear Combining . . . . . . . . . . . . . 151

6.3 Downlink Performance Analysis . . . . . . . . . . . . . . 152

6.3.1 MF Precoding Analysis . . . . . . . . . . . . . . 152

6.3.2 Zero-Forcing Precoding Analysis . . . . . . . . . . . . . . 157

6.4 Uplink Analysis With I.I.D. Rayleigh Fading . . . . . . . . . . . 159 
6.4.1 Maximum-Ratio Combining Analysis . . . . . . . . . . . 159

6.4.2 Zero-Forcing Combining Analysis . . . . . . . . . . . 161

6.4.3 Minimum-Mean-Squared-Error Combining Analysis .................... . . 162

6.4.4 Extensions to More General Fading Channels . . . . . . 164

6.5 Numerical Results and Discussion . . . . . . . . . . . . . 165

6.6 Concluding Remarks . . . . . . . . . . . . . . . . . . . 173

7 Linear Precoding Analysis With Unequal Spatial Correlation 175

7.1 Introduction . . . . . . . . . . . . . . 176

7.2 Typical Spatial Correlation Scenarios . . . . . . . . . . . . . 180

7.3 System Model . . . . . . . . . . . . . . . . . 182

7.3 .1 Signal Model . . . . . . . . . . . . . . . . . . 182

7.3.2 MF SINR, ZF SNR and Ergodic Sum Spectral

Efficiency .................. 184

7.4 Analysis Methodology and Implications . . . . . . . . . . . 185

7.4.1 Expected MF SINR and Ergodic Sum Spectral Efficiency 185

7.4.2 Expected ZF SNR and Ergodic Sum Spectral

Efficiency . . . . . . . . . . . . . . . 190

7.5 Numerical Results . . . . . . . . . . . . . . . . . . . . . . 194

7.5.1 Spatial Correlation Models . . . . . . . . . . . . . . . 194

7.5.2 Performance of Unequal vs. Equal Spatial Correlation . . . . . . . . . . . . 196

7.6 Conclusion . . . . . . . . . . . . . . . . . . 201

8 Uplink MRC Analysis With Fixed Physical Array Sizes 203

8.1 Introduction . . . . . . . . . . . . . . . 204

8.2 System Model . . . . . . . . . . . . . . . . . . . . . 207

8.2.1 Signal Model . . . . . . . . . . . . . . . 207

8.2.2 Proposed Space-Constrained Channel Model . . . . . . . 208

8.2.3 SINR and Ergodic Sum Spectral Efficiency . . . . . . . . 210

8.3 Expected SINR and Ergodic Sum Spectral Efficiency Analysis . 211 
8.4 Special Cases . . . . . . . . . . . . . . . . . . . . . . . . . . . 218

8.5 Limiting Expected SINR and Ergodic Sum Spectral Efficiency Analysis . . . . . . . . . . . . . . . . 220

8.6 Numerical Results and Discussion . . . . . . . . . . . . . . 225

8.7 Concluding Remarks . . . . . . . . . . . . . . . . . . . . 231

9 Conclusions and Future Work 233

9.1 Thesis Summary . . . . . . . . . . . . . . . . . . . 233

9.2 Chapter Summary and Concluding Remarks . . . . . . . . . . . 234

9.3 Future Work . . . . . . . . . . . . . . . . . . . . . . . . . . . . 238

9.3.1 MU-MIMO Analysis With Spatial Channel Models . . . 239

9.3.2 Terminals vs. Pilots for Large MU-MIMO Systems . . . 240

9.3.3 Large MU-MIMO With Double Scattering . . . . . . . . 242

9.4 Final Remarks . . . . . . . . . . . . . . . . . . . . . . . 243 


\title{
List of Symbols and Operators
}

\author{
$x$ \\ Scalar value of $x$ \\ $\boldsymbol{x} \quad$ The vector $\boldsymbol{x}$ \\ $\begin{array}{ll}\boldsymbol{X} & \text { The matrix } \boldsymbol{X}\end{array}$ \\ $[\boldsymbol{X}]_{x, y} \quad$ The $(x, y)$-th entry of the matrix $\boldsymbol{X}$ \\ $[\boldsymbol{X}]_{. ; x} \quad$ The matrix $\boldsymbol{X}$ with column $x$ removed \\ $[\boldsymbol{X}]_{x ; y} \quad$ The matrix $\boldsymbol{X}$ with row $x$ and column $y$ removed \\ $[\boldsymbol{X}]_{a, b ; c, d} \quad$ The matrix $\boldsymbol{X}$ with rows $a, b$ and columns $c, d$ removed \\ $\boldsymbol{I}_{X} \quad$ An identity matrix with dimensions $X \times X$ \\ $\operatorname{diag}(\boldsymbol{X}) \quad$ The matrix $\boldsymbol{X}$ with only diagonal entries \\ $\operatorname{det}[\boldsymbol{X}] \quad$ The matrix determinant of $\boldsymbol{X}$ \\ $\operatorname{tr}[\boldsymbol{X}] \quad$ The matrix trace of $\boldsymbol{X}$ \\ $\operatorname{rank}(\boldsymbol{X}) \quad$ Rank of the matrix $\boldsymbol{X}$
}


$|\cdot| \quad$ Absolute value of a complex number

$\|\cdot\| \quad$ Euclidean norm of a vector

$\|\cdot\|_{\mathrm{F}} \quad$ Frobenius norm of a matrix

$(\cdot)^{*} \quad$ Conjugation of a complex number

$(\cdot)^{-1} \quad$ Inverse of a matrix or a scalar

$(\cdot)^{\mathrm{T}} \quad$ Transposition of a vector or matrix

$(\cdot)^{\mathrm{H}} \quad$ Conjugate transposition of a vector or matrix

$\mathbf{0}_{X \times X^{\prime}} \quad X \times X^{\prime}$ matrix of zeros

$\mathbf{1}_{X \times X^{\prime}} \quad X \times X^{\prime}$ matrix of ones

$\mathbb{E}[X] \quad$ Statistical expectation of the random variable $X$

$\operatorname{Var}[X] \quad$ Variance of the random variable $X$

$\operatorname{Cov}[X, Y] \quad$ Covariance of the random variables $X$ and $Y$

$\sim \quad$ Reads "distributed as"

$\mathcal{C N}(\cdot, \cdot) \quad$ Complex Gaussian distribution

$\mathcal{U}[\cdot, \cdot] \quad$ Uniform distribution

$x ! \quad$ Factorial of a real scalar $x$ 


\begin{tabular}{|c|c|}
\hline$\left(\begin{array}{l}x \\
y\end{array}\right)$ & Binomial co-efficient indexed by $x$ and $y$ \\
\hline $\mathcal{X} \in \mathcal{Y}$ & $\mathcal{X}$ takes values from the set $\mathcal{Y}$ \\
\hline$\infty$ & Infinity \\
\hline$\forall$ & Reads "for all" \\
\hline $\operatorname{per}(x)$ & Permutations of $x$ \\
\hline $\min (\boldsymbol{x})$ & Minimum entry in the vector $\boldsymbol{x}$ \\
\hline $\max (\boldsymbol{x})$ & Maximum entry in the vector $\boldsymbol{x}$ \\
\hline $\arg \min (\boldsymbol{x})$ & Index of the minimum entry in the vector $\boldsymbol{x}$ \\
\hline $\arg \max (\boldsymbol{x})$ & Index of the maximum entry in the vector $\boldsymbol{x}$ \\
\hline $\lim \{f(\cdot)\}$ & Limiting value of a function $f(\cdot)$ \\
\hline$\lfloor x\rfloor$ & Floor function of $x$ \\
\hline
\end{tabular}

All other notation and symbols are defined in the text for each chapter. 


\section{Chapter 1}

\section{Introduction}

\subsection{Motivation Statement}

Given the vast extent of the electromagnetic spectrum, at first sight, it would be rather preposterous to believe that the spectral efficiency offered by the wireless medium is limited. To draw a simile, it is like somebody sitting in a small shelter in Wellington, New Zealand, and claiming that there is not enough space in our universe. From an unimaginable quantity of electromagnetic spectrum, auctioning 65 Megahertz $(\mathrm{MHz})$ of bandwidth for $\$ 41.3$ billion on the grounds of scarce spectrum seems absurd. Such an auction happened in 2015 in the United States of America (formally known as auction number 97), administered by the Federal Communications Commission for spectrum allocated to advanced wireless services [1]. The impact of this auction is now spreading globally, with other countries following the same trend and auctioning spectrum for wireless services.

These developments are being driven by a new attitude in society. We are at the beginning of an era where humans have begun to understand what freedom of information means and have grasped the idea of having access to any information, anywhere, at anytime, anyhow, at any cost. This has resulted in a global exponential demand for wireless data, which is expected to increase to 15.9 exabytes per month by 2018, resulting in a 6-fold increase over $2014[2,3]$. Keeping in view the freedom of communication we are able to achieve in just 
$65 \mathrm{MHz}$ of bandwidth, if much larger bandwidths are to be considered e.g., 650 $\mathrm{MHz}$, possibilities for future communications are limitless.

Looking into the future, the way one is seeing the electromagnetic spectrum is changing. There is a growing push to operate future wireless (cellular) systems at carrier frequencies much higher than those in use today, such as in the millimeter-wave (mmWave) frequency band. Here, even higher bandwidths than those quoted above can be exploited. This line of thinking demonstrates that the wireless medium, itself, is not be the data bottleneck as it is frequently claimed. Instead, it is the physical implications of operation at these frequencies, which limits the resulting performance of the system. For instance, the high electromagnetic losses at mmWave carrier frequencies implies that the radio waves are unable to propagate very far, needing a large number of antennas to overcome these losses. Naturally, these antennas have to be placed in confined volumes with relatively small spacing between the adjacent elements. Furthermore, the inability of the radio waves to propagate large distances may lead the system to serve a commensurately smaller coverage area, creating line-of-sight (LoS) components in the propagation channel. Overall, in contrast to lower carrier frequencies, the fusion of the above physical phenomena creates a heterogeneous propagation environment. Such types of environments are increasingly likely to occur in future cellular systems with large bandwidths, making their performance analysis timely, and of vital importance. This is the aim of the thesis.

According to the fundamental limits of reliable communication laid out by Shannon in 1948, a single-antenna cellular link offers a spectral efficiency of approximately $3.5 \mathrm{bits} / \mathrm{seconds} / \mathrm{Hz}$ (bits/sec/Hz) at a link signal-to-noise-ratio (SNR) of 10 decibels (dB) [4]. Such a spectral efficiency is rather poor for high data rate services and is clearly not a viable solution for the future. On the other hand, the more recently investigated point-to-point multiple-input multiple-output (MIMO) systems promise to meet the high spectral efficiency of up to several tens of bits/sec/Hz, dependent on the communication scenario [5-7]. Of course, this comes at a price of complex transceiver structures, which 
need to be designed according to the operating conditions. Nevertheless, these costs fall well short in comparison to the potential spectral efficiency gains on offer. Enhancing the reliability of wireless links, MIMO systems rely on spatial diversity to combat the effects of fading in the propagation channel. By transmitting multiple parallel streams of data on the same frequency, MIMO systems are known to leverage a linear gain in the spectral efficiency as a function of the minimum number of transmit or receive antennas. Since the landmark work of Foschini, Gans, Teletar, and Winters [5-7], MIMO systems have gained significant attention, and are incorporated into several wireless broadband standards, such as Long Term Evolution-Advanced (LTE-A), IEEE 802.11n, and IEEE 802.11ac [5-7]. Having said the above, the multiplexing gains of a point-topoint MIMO system may disappear near the edge of the transmitter coverage area, where the desired signal levels are low relative to the interference, or in a propagation environment which is insufficiently dominated by scattering $[8,9]$.

As a result, the effort to retain the spatial multiplexing gains of a pointto-point MIMO system has shifted to a multiuser MIMO (MU-MIMO) system where an antenna array, often at a cellular base station (BS), simultaneously serves multiple user terminals inside the BS coverage area $[8,10]$. These terminals are usually low-cost, single-antenna devices, and the multiplexing gains of the system are shared amongst the terminals. Furthermore, a MU-MIMO system is far more tolerant of the propagation environment than a point-to-point MIMO system [9,11]. For instance, under LoS propagation, multiplexing gains can disappear for a point-to-point MIMO system, but are retained for MUMIMO systems provided that the angular separation of the terminals exceeds the spatial resolution of the antenna array. Nevertheless, serving multiple terminals simultaneously comes at a further cost of multiuser interference, causing the performance of a given terminal, and the system as a whole to degrade significantly. To overcome this issue, transceiver structures which reduce or cancel multiuser interference are employed at the transmit or receive end of the link.

The increasing interest to operate future cellular systems in the mmWave band requires the deployment of large antenna arrays serving smaller areas 
$[9,12,13]$. With the BS array being in closer vicinity to the terminals, the fundamental propagation characteristics are likely to be more heterogeneous. As more antenna elements are added to a fixed antenna array form factor, spatial separation between antenna elements reduces consequently inducing spatial correlation in the channel. Moreover, the reduction in average link the distance between the BS and the terminal may lead to less scattered propagation environments. Here, the impact of dominant LoS directions may significantly influence the channel impulse response. The effects of the above mentioned phenomena are likely to be unique for different terminals located in different parts of the coverage area, due to the variability in the amount of local scattering around each terminal. To this end, the propagation channels to two terminals can be significantly different with unequal spatial correlation patterns and LoS directions. In addition to this, each channel also has a unique large, and small-scale power variation, as a result of the terrain variations across multiple terminals.

A combination of all the above physical artifacts is rather difficult to handle mathematically, when analyzing the performance of MU-MIMO system with linear transceiver structures. This is due to the fact that most performance metrics involve manipulation of the instantaneous channel correlation matrix, which have extremely complex structures in heterogeneous propagation environments. As the dominating theme of future cellular systems is likely to be the interplay of small-cellular systems and large antenna arrays, it is imperative to have a fundamental understanding of MU-MIMO systems with variations in spatial correlation patterns, LoS directions, small-scale, and large-scale powers.

In general, performance analysis of such systems is critical in gaining an in-depth understanding into the operation of MU-MIMO systems under heterogeneous propagation channels. To this end, performance metrics such as the terminal signal-to-interference-plus-noise-ratio (SINR) and ergodic sum spectral efficiency are often discussed in the literature, which help characterize the performance of current and future MU-MIMO systems $[14,15]$. It is in the characterization of such performance metrics that exciting opportunities lie for novel mathematical advances, allowing one to gain a deeper understanding into the 
sensitivities of MU-MIMO performance under extremely general fading conditions. This constitutes the focus of the thesis.

In the following section, the problem statement and the focus of the thesis are presented.

\subsection{Problem Statement and Focus of the Thesis}

Performance results for SISO systems are well known for a wide variety of scenarios [16]. However, performance analysis of point-to-point MIMO systems are fundamentally different from SISO systems, due to the higher dimensionality of the propagation channel and its associated physical artifacts [17], as explained in Chapter 2 of the thesis. Hence, performance analyses of MIMO systems have been executed in parallel with linear algebra and multivariate statistical techniques, which have directly influenced cellular research over many decades [18-20]. As these theories have established coherent methodologies, performance analyses of many conventional point-to-point MIMO systems are now also well known $[5,14,21-26]$.

In contrast to this, performance analysis of MU-MIMO systems under heterogeneous channel conditions is extremely difficult, due to the lack of a coherent and well established framework to handle the wide range of the propagation channels which could arise in MU-MIMO systems. Naturally, numerical evaluation of such systems is always possible, however, an enormous drawback of numerical performance evaluation is that it does not allow one to efficiently identify the most influential system and propagation parameters that contribute to the end performance. An understanding of these contributing factors is imperative, as it allows one to gain a more intuitive insights into the specific parameters which have a strong impact on system performance. Furthermore, analytical results allow greater numerical stability, accuracy, and are vital in providing a theoretical basis to which the numerically simulated performance could be compared. In addition to the above, analytical results provide improvements in the speed and help to reduce the number of number of computations required to 
characterize the system performance.

Keeping the above in mind, analytical results for the SINR and ergodic sum spectral efficiency are invaluable for understanding optimized design and rapid evolution of MU-MIMO systems. Hence, the prime focus of this thesis is to obtain analytical results for the performance of MU-MIMO systems with linear transceiver structures. As such, the thesis contains rigorous investigations into the performance of MU-MIMO systems in generalized fading channels and scenarios which are relevant to current and future cellular architectures. This leads to the main contributions of the thesis.

\subsection{Main Contributions of the Thesis}

Despite the lack of statistical approaches to deal with the generality of propagation channels which are likely to occur in MU-MIMO systems, the thesis is able to analyze several practically important MU-MIMO scenarios, quantifying the link level performance. The results contained in the thesis develop a rigorous analytical framework for the analysis of finite and large MU-MIMO systems with linear transceivers, and have led to solutions of several long-standing research problems. In particular, the major contributions of the work are as follows:

\subsubsection{Coordinated RZF Precoding in Multi-Cellular Systems}

Chapter 4 of the thesis demonstrates the impact of BS coordination on the performance of multicellular, MU-MIMO systems. Here, a general analysis methodology is developed to evaluate the expected (average) SINR of a given terminal and the ergodic sum spectral efficiency of a given cell, with coordinated regularized zero-forcing (RZF) precoding. Application to two-tier small-cellular networks is considered, assuming independent and identically distributed (i.i.d.), as well as semi-correlated Rayleigh fading channels, with spatial correlation at the BS array. 
With semi-correlated Rayleigh fading, the generalized analysis methodology caters for the presence of equal and unequal spatial correlation matrices. With i.i.d. Rayleigh fading, and when each terminal is assigned an equal correlation matrix, the expressions are averaged over the arbitrary and over a joint pair of arbitrary eigenvalue densities of the instantaneous channel correlation matrix, which follows an uncorrelated and correlated complex central Wishart distribution. The correlated complex central Wishart distributed eigenvalue densities were previously unknown and are derived in Chapter 2 of the thesis. For unequal correlation matrices, the RZF performance is approximated with zeroforcing (ZF) precoding and insightful closed-form expressions are derived for the expected ZF SNR and ergodic per-cell sum spectral efficiency. The numerical results indicate the superiority of network-wide BS coordination, in comparison to cell-wide and macro-only BS coordination strategies.

The impact of network densification on a given terminal and a given cell is explored with uniform, cell-edge and cell-centric small-cell placements. The performance of such systems with unequal spatial correlation is found to be superior to that where each terminal is assigned a fixed correlation matrix. The derived approximations are shown to be robust to variation in the system dimensions, link SNRs and unequal levels of spatial correlation. With a general analysis methodology, the results derived are applicable to other types of fading channel models, and provide further advancements to the area of random matrix theory.

\subsubsection{RZF Precoding in Ricean Fading}

With the emergence of small-cellular systems, a serving BS is more likely to be in proximity of a given terminal. As a result of this, LoS in the propagation channel from the BS to the terminal is likely to dominate future cellular access. In chapter 5, the impact of dominant LoS directions on the terminal SINR and system spectral efficiency is investigated. Statistically, a Ricean fading channel model is employed to model the presence of LoS, along with many scattered components in the propagation channel. With RZF precoding on the down- 
link, analytical expressions which approximate the expected per-terminal SINR and ergodic sum spectral efficiency are derived. The derived expressions are averaged with respect to the previously unknown arbitrary eigenvalue densities of the complex non-central Wishart distributed instantaneous channel correlation matrix. To aid the derivation of the expected SINR, analytical expressions derived in Chapter 2 for the joint density of two arbitrary eigenvalues of the complex non-central Wishart matrix are employed. Unlike previous studies, a unique Rice factor is modeled for each terminal where the considered system model caters for terrain variations across multiple terminals, making it suitable for analysis of future systems.

In the high link SNR regime, an approximation to the distribution of the instantaneous RZF SINR is developed with ZF precoding. It is shown that the ZF SNR is well approximated by the gamma distribution, whose parameters are derived. The presented numerical findings suggest that while the presence of dominant LoS has an adverse effect on the expected RZF SINR, RZF ergodic sum spectral efficiency and instantaneous ZF SNR, increasing the Rice factor variability tends to enhance the peak RZF ergodic sum spectral efficiency and ZF SNR. Both the developed approximations are insensitive to changes in the system dimensions, link SNRs, and unequal levels of LoS, respectively.

\subsubsection{Simplified Analysis With Linear Transceivers}

As large antenna arrays are expected to feature prominently in future cellular systems, it is likely to push MU-MIMO systems to an extreme. Nevertheless, in order to retain wide area coverage capability in the medium-term future, the scenario with moderate numbers of BS antennas operating at microwave frequencies is also of significant interest. Motivated by this, under i.i.d. Rayleigh fading, Chapter 6 develops a simplified analysis methodology for a general class of linear transceivers. More specifically, the instantaneous and expected perterminal SINR, SNR, as well as the instantaneous and ergodic sum spectral efficiency is analyzed with matched-filter $(\mathrm{MF})$ and ZF precoding on the downlink, and maximum-ratio combining (MRC), ZF, and minimum-mean-squared-error 
combining on the uplink. The approximation methodology is based on the identification of specific components in the above mentioned performance metrics which tend to stabilize for moderate numbers of BS antennas, and replacing only these specific components by their expected values.

In general, the resulting expressions allow for clear insights into the factors which most contribute to the above mentioned performance metrics. Numerical evaluation of the approximations demonstrate they are extremely accurate and stable for moderate numbers of BS antennas. Moreover, the approximation methodology is robust to the type of fading environment assumed, and easily extendable to suit other statistical channel models, such as semi-correlated Ricean fading.

\subsubsection{Linear Precoding Analysis With Unequal Spatial Correlation}

For downlink MU-MIMO systems, it is well known that spatial correlation across an antenna array is detrimental to the SINR, and in turn the system spectral efficiency. Chapter 7 demonstrates that the widely used, yet overly simplified, spatial correlation models that result in common correlation structures for each terminal tend to underestimate the MU-MIMO system performance. As such, such models act as a lower bound on the resulting performance. In vast contrast to this, the use of more complex, yet physically motivated, remote scattering models result in unequal spatial correlation structures for each terminal, leading to higher performance. The main aim of Chapter 7 is to provide a mathematical quantification of the performance difference with equal and unequal spatial correlation matrices in a multiuser environment.

Assuming semi-correlated Rayleigh fading with MF and ZF processing, closedform approximations to the expected SINR, expected SNR, and ergodic sum spectral efficiency of a MU-MIMO system are derived. The derived expressions provide clear insights into the impact of unequal spatial correlation matrices. The expressions are robust to changes in the system dimensions, link SNRs, and 
unequal spatial correlation levels. Moreover, the derived approximations remain accurate for all non-physical and physical correlation models considered.

Overall, the results from this chapter demonstrate the sensitivity of the resulting MU-MIMO performance using different spatial correlation models. Such a sensitivity analysis is extremely valuable in providing a cautionary tale of its impact to researchers.

\subsubsection{Uplink MRC Analysis With Fixed Array Sizes}

In contrast to many prior studies which consider fixed inter-element spacing between two adjacent antennas at the BS, and therefore, assume an increasing array aperture with growing number of elements, fixed physical array sizes are considered in Chapter 8. Here, an increase in the number of antennas in a fixed space imposes an inversely proportional decrease in the inter-element spacing. The chapter analyzes the uplink performance of large MU-MIMO systems, where the BS antennas are configured in a uniform linear array (ULA) with a fixed total space.

An investigation into the combined effects of two contradicting phenomena is carried out: First, the reduction of spatial diversity due to reducing the spatial separation between the BS elements, and second, the increase in receive diversity by increasing the sheer number of elements. In this context, with MRC processing at the BS, the chapter presents a general analytical framework for approximating the ergodic sum spectral efficiency, by approximating the expected per-terminal SINR. The chapter extends and generalizes a prior space-constrained channel model, by including the effects of unequal levels of receive spatial correlation and unequal levels of LoS for each terminal. Deterministic limits of the SINR and sum spectral efficiency are also analyzed, when the number of BS antennas are increased indefinitely with a fixed number of terminals.

The derived analytical expressions lead to numerous useful insights into the behavior of the expected SINR and ergodic sum spectral efficiency with variations in the system and propagation parameters. Supporting numerical results 


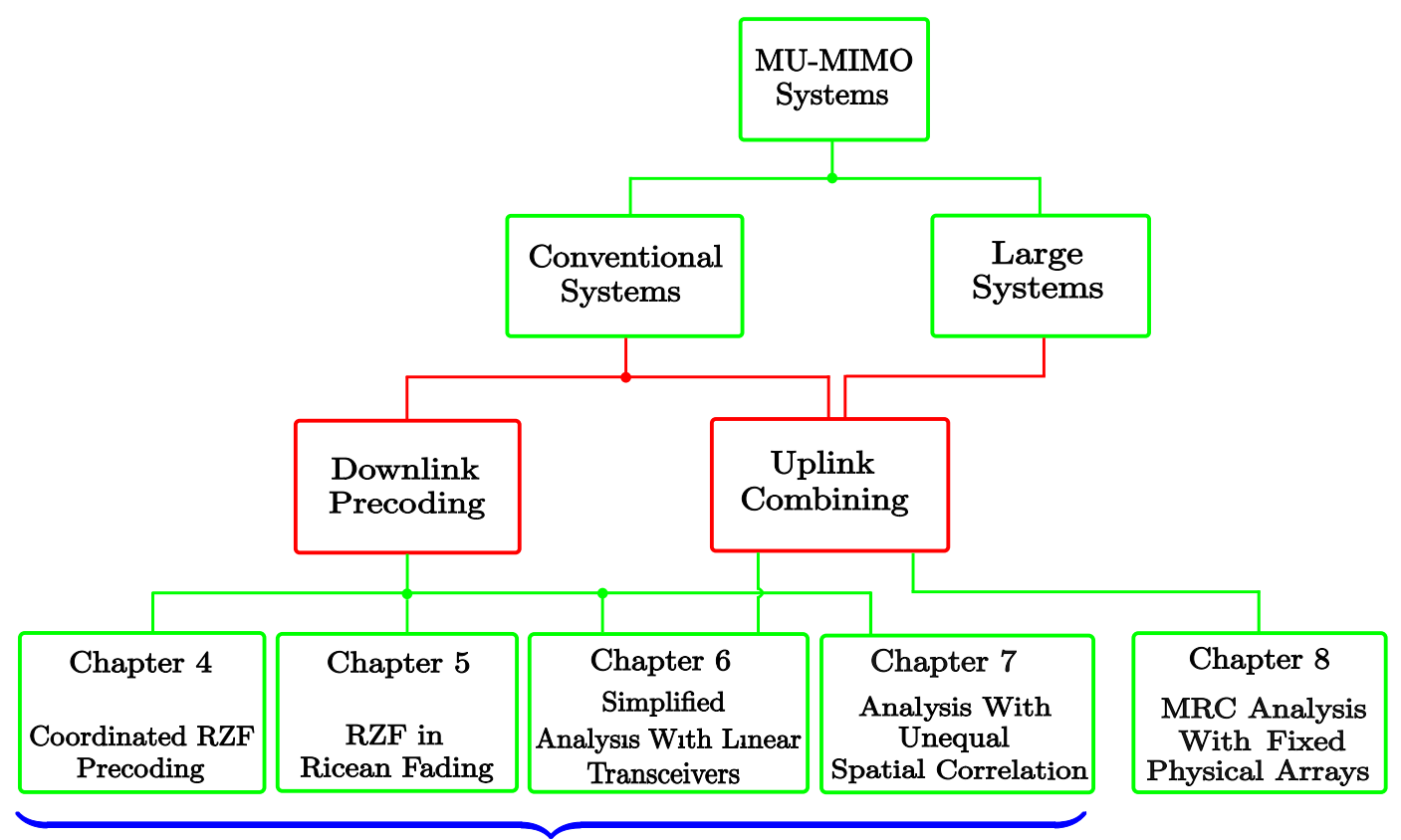

Also Directly Applicable to Large Systems

Figure 1.1: Overall structure of the thesis.

demonstrate that a SC ULA deployment with MRC processing causes saturation of the expected SINR and ergodic sum spectral efficiency with increasing link SNR and serving antennas. Several special cases of the derived expressions are also presented when only non LoS components are present with equal and unequal spatial correlation matrices, as well as when each terminal having LoS components has equal correlation matrices.

The major contributions of the thesis can also be easily followed from the graphic presented Fig. 1.1. This concludes the major contributions of the thesis, and leads to the outline of the thesis.

\subsection{Thesis Outline}

Subsequent to the introduction chapter, the thesis is organized following the structure.

Chapter 2 provides a detailed overview of point-to-point MIMO systems, along with the fundamentals of wireless channels, for the purpose of presenting novel random matrix theory results on the joint pair of arbitrary eigenvalues of 
complex correlated central and non-central Wishart matrices. The newly derived random matrix results are then utilized in Chapters 4 and 5, in analyzing the expected per-terminal SINR and ergodic sum spectral efficiency under semicorrelated Rayleigh and uncorrelated Ricean fading channels, respectively.

Chapter 3 introduces the notion of a MU-MIMO system, and provides a comprehensive review of multiuser systems and the relevant performance metrics. In particular, this chapter describes the difference between point-to-point MIMO and MU-MIMO propagation channels, as well detailing the necessary background in linear transmit and receive signal processing techniques. Such techniques are an integral part of current and future cellular systems, as they provide the ability to serve multiple terminals in the same time-frequency resource, while reducing the resultant multiuser interference. These techniques are also frequently used to analyze the performance sensitivities in MU-MIMO performance and constitute the foundation of the thesis.

Chapter 4 presents the impact of BS coordination on multicellular, MUMIMO systems. A novel analytical methodology is demonstrated where the performance of coordinated RZF precoding is presented under i.i.d. and semicorrelated Rayleigh fading, with spatial correlation at the BS array. Both equal and unequal spatial correlation matrices to each terminal are considered. Extensive numerical results demonstrate the tightness of the derived analysis and show the performance of different BS placement and coordination strategies on a per-terminal and system basis. The performance with equal correlation matrices is found to be lower than that with unequal correlation matrices.

Chapter 5 extends the ideas presented in Chapter 4, and demonstrates the impact of dominant LoS components in the propagation channel, on the RZF expected SINR and ergodic sum spectral efficiency. With Ricean fading channels, propagation parameters from both microwave and mmWave frequencies are considered, with a unique Rice factor for each terminal. The instantaneous RZF SINR distribution is approximated in the high link SNR regime and its relevant parameters are derived. Rather interestingly, increasing the Rice factor variability led to an increase in the peak performance of the system, while having 
an opposite effect at the cell-edge reducing the performance of the system.

Chapter 6 presents simplified approximations to analyze the downlink and uplink performance of MU-MIMO systems, for a general class of linear transceiver structures operating in i.i.d. Rayleigh fading channels. All the derived expressions are shown to be numerically stable and accurate for moderate numbers of BS antennas. The approximations offer direct insights into the most influential system and propagation parameters contributing to the per-terminal and system performance. Even though the presented analysis methodology is simple, it is shown to be easily adaptable to other types of statistical channel models, amplifying the generality of the approximation approach.

Chapter 7 presents the impact of unequal spatial correlation structures in MU-MIMO systems. With both MF and ZF precoding, on the downlink of a semi-correlated Rayleigh fading channel, it is mathematically proven that unequal spatial correlation structures yields higher performance in comparison to equal correlation matrices. Tight closed-form expressions to the expected SINR and ergodic sum spectral efficiencies are derived. The derived analytical expressions are extremely insightful, simple, and provide a clear indication of the main performance contributing factors. The results from this chapter demonstrate that the MU-MIMO system performance is ultimately governed by the correlation structure present in the propagation channel. The results from this chapter provide a sensitivity analysis into the impact of using different spatial correlation models.

Chapter 8 of the thesis presents an investigation into space-constrained ULAs, operating under spatially correlated Ricean fading environments. Under the most general propagation conditions, with unequal levels of LoS, unequal link gains, and unequal spatial correlation matrices for each terminal, the chapter presents closed-form analysis of expected SINR and ergodic spectral efficiency with uplink MRC processing. The chapter extends the traditional space-constrained channel model and presents clear insights into the impact of unequal LoS and spatial correlation levels. Deterministic limits to the terminal SINR and sum spectral efficiency are also derived with an increase in the num- 
ber of BS antennas and fixed number of user terminals. Supporting numerical results show that a space-constrained deployment with MRC leads to a saturation of the expected SINR and ergodic sum spectral efficiency with increasing link SNR and BS antennas.

Finally, Chapter 9 presents the concluding remarks of the thesis by summarizing each chapter and the conclusions derived throughout. Furthermore, future research directions within the framework of the thesis are also presented.

This concludes the outline of the thesis, leading to the publications which have resulted from the work carried out in the thesis.

\subsection{Publications}

The work developed throughout the thesis has resulted in the following publications:

- H. Tataria, P. J. Smith, P. A. Dmochowski, and M. Shafi, "General Analysis of Multiuser MIMO Systems With Regularized Zero-Forcing Precoding Under Spatially Correlated Rayleigh Fading Channels", in Proceedings of IEEE the International Conference on Communications (ICC), Pages 2582 - 2588, Kuala Lumpur, Malaysia, May 2016.

- H. Tataria, P. J. Smith, L. J. Greenstein, P. A. Dmochowski, and M. Shafi, "Performance and Analysis of Downlink Multiuser MIMO Systems With Regularized Zero-Forcing Precoding in Ricean Fading Channels", in Proceedings of the IEEE International Conference on Communications (ICC), Pages 1185 - 1192, Kuala Lumpur, Malaysia, May 2016.

- H. Tataria, P. J. Smith, L. J. Greenstein, and P. A. Dmochowski, "ZeroForcing Precoding Performance in Multiuser MIMO Systems With Heterogeneous Ricean Fading", IEEE Wireless Communications Letters, Volume 6, Issue 1, Pages $74-77$, February 2017.

- H. Tataria, P. J. Smith, and P. A. Dmochowski, "On the General Analysis of Coordinated Regularized Zero-Forcing Precoding: An Application to 
Two-Tier Small-Cell Networks", IEEE Transactions on Communications, vol. PP, no. 99, Pages 1 - 17, April 2017.

- H. Tataria, P. J. Smith, M. Matthaiou, and P. A. Dmochowski, "Uplink Analysis of Large MU-MIMO Systems With Space-Constrained Arrays in Ricean Fading, in Proceedings of the IEEE International Conference on Communications (ICC), Pages 1 - 7, Paris, France, May 2017.

- H. Tataria, P. J. Smith, L. J. Greenstein, P. A. Dmochowski, and M. Matthaiou, "On the Impact of Line-of-Sight and Unequal Spatial Correlation in Uplink MU-MIMO Systems", IEEE Wireless Communications Letters, vol. PP, no. 99, Pages 1 - 4, July 2017.

- H. Tataria, P. J. Smith, A. F. Molisch, L. J. Greenstein, S. Sangodoyin, J. Zhang, R. S. Thomä, P. A. Dmochowski, and M. Matthaiou, "Impact of Unequal Spatial Correlation Patterns on Downlink MU-MIMO: Analysis, Measurements, and Models", IEEE Transactions on Wireless Communications, Pages 1 - 16, July 2017 (To be Submitted).

- H. Tataria, P. J. Smith, P.A. Dmochowski, and M. Matthaiou, "Uplink MMSE Analysis in Spatially Correlated Ricean Fading for Massive MIMO Systems", IEEE Transactions on Vehicular Technology, Pages 1 - 5, July 2017 (To be Submitted). 


\section{Chapter 2}

\section{Statistical Channel Models and Random Matrix Theory}

Summary. The performance of cellular communication systems is ultimately governed by the propagation channels they operate in. It is thus vital to investigate the channel characteristics that are relevant to the types of cellular systems considered in the thesis. In this chapter, an overview of the typical propagation scenarios and their relevant statistical models are presented. Both single-input single-output (SISO) and multiple-input multiple-output (MIMO) cellular channels are considered. Many variations on the well known independent and identically distributed Rayleigh fading channel model are considered, with the presence of strong transmit spatial correlation, and line-of-sight (LoS) directions. Furthermore, the spatial correlation properties of MIMO channels are also statistically characterized, where novel analytical results concerning the distribution of the arbitrary, and a joint pair of arbitrary eigenvalues are derived, for a general class of correlated and uncorrelated Wishart matrices. The derived results can be directly applied to predict the spectral efficiency of MIMO channels, and have many other applications, such as the characterization of multiuser signal and interference powers, as demonstrated in Chapters 4 and 5 of the thesis. Other applications of interest are also listed in the chapter. An extensive numerical comparison of channel correlation matrix eigenvalue densities is presented with variations in the MIMO system size, LoS strengths, and link 
signal-to-noise-ratio levels. The numerical results allow one to draw many useful insights into the behavior of conventional and large MIMO channels, reinforcing the fact that the ultimate performance of such systems is directly a function of the assumed propagation characteristics. Overall, the chapter contains both the background material on SISO and MIMO channels, as well as novel results in random matrix theory. Due to the broad scope of the work, this chapter is the first of two (along with Chapter 3) offering a broader background of the research related to the contributions of the thesis. Further details on each specific area are also provided in the relevant chapters. ${ }^{1}$

\subsection{Wireless Channels and Models}

It is worth noting that many types of wireless channel characterizations are available for digital cellular communications [27], indoor communications [28], and over-the-horizon communications [29], respectively. However, unlike additive white Gaussian noise channels [16], cellular channels suffer from a fundamentally different physical artifact known as fading, which creates a new set of engineering challenges that must be considered in the design of such communication systems. In this thesis, digital cellular channels are primarily considered in outdoor environments. As a result, a brief overview of the typical multipath propagation environment is presented in the following section.

\subsubsection{A Typical Multipath Environment}

In a cellular system, transmitted signals arrive at a mobile receiver via various propagation mechanisms, over multiple propagation paths (known as multipath), each with different time-varying delays, directions-of-departure (DoDs),

\footnotetext{
${ }^{1}$ Specific to this and the next chapter (Chapters 2 and 3 ) of the thesis, in-depth "Introduction" and "Concluding Remarks" are omitted, since the chapters aim to present the necessary background material needed to understand the contributions of the thesis. In all other subsequent chapters, i.e., Chapters 4-8, chapter-wise "Introduction" and "Concluding Remarks" sections are presented. For the same reason, Chapters 2 and 3 also omit an explicit section on Numerical Results, as they are presented in-line with the text where applicable. For all other chapters, i.e., Chapters 4-8, a specific section on "Numerical Results" is presented to evaluate the performance of the considered system model.
} 
directions-of-arrivals (DoAs), random phases, and attenuations, giving rise to a highly complex propagation channel. The possible physical propagation mechanisms can be categorized into five types: (1) line-of-sight (LoS) propagation, (2) absorption, (3) specular reflection, (4) diffraction, and (5) diffusion (also known as diffuse scattering). ${ }^{2}$ With the exception of LoS, all of these physical mechanisms imply interaction of the propagating wave with one or more arbitrary obstacles, such as walls, trees, cars, human beings, etc. In this thesis, these obstacles will be referred to as scatterers. Usually, the LoS path only undergoes free-space loss (geometric attenuation), while a specular reflection occurs when the propagating wave impinges upon a smooth or a plane surface whose dimensions are much larger in comparison to the wavelength of the transmitted signal. Furthermore, diffraction appears when the propagating wave is obstructed by a discontinuity, such as an edge of a surface. Transmission through an obstacle also causes partial absorption of electromagnetic energy, and diffusion is caused by interactions of the propagating wave with objects having dimensions comparable to the signal wavelength, e.g., foliage or rough surfaces.

Due to the above artifacts, the power at a receive antenna randomly varies, according to two broad classifications: (1) large-scale fading, which encompasses the effects of distance based geometric attenuation, as well as shadow-fading, occurring from blockages in the propagation paths between the transmitter and the receiver. With large-scale fading, the average received power varies as a function of the transmitter-receiver separation distance. (2) Small-scale fading (also known as multipath fading), where rapid fluctuations occur in the instantaneous signal strength around the mean power, at a particular transmitter-receiver separation distance. Due to mobility of either the transmitter, the receiver and/or the scatterers in the propagation channels, the wireless channel is a time varying system. As a result, the strength of the received signal also varies with time, along with the geometric attenuation, shadow-fading and multipath fading. ${ }^{3}$

\footnotetext{
${ }^{2}$ Note that in addition to these, any combination of the last four propagation mechanisms is also possible in a typical multipath environment.

${ }^{3}$ This is a particularly important point for wideband systems, where each delay in the propagation channel at a particular time instance is resolvable.
} 
As a result of the large number of influencing factors on a typical cellular channel, physical modeling of a generic wireless link is rather difficult. There exists a vast amount of literature in this area with many industrial standards, where cellular channel models appear (see e.g., [14,28,30-32] and references therein). Nevertheless, in this thesis, simple, yet widely accepted stochastic modeling approaches are considered [33]. These are as discussed in Section 2.1.3 for single-input single-output (SISO) and Section 2.3 for multiple-input multipleoutput (MIMO) systems, respectively. Furthermore, a comparison between the industrial spatial channel models and simple stochastic channel models can be found in [34].

In the sections which follow, statistical models for the characterization of large-scale and small-scale fading are presented.

\subsubsection{Large-Scale Fading}

The geometric attenuation of the transmitted waveform is often modeled via ${ }^{4}$

$$
\beta=v\left(\frac{d_{0}}{d}\right)^{\alpha}
$$

where $\beta$ is known as the link gain. More specifically, $\alpha$ is the attenuation exponent, and $v$ is the unit-less constant for geometric attenuation at a reference distance $d_{0}$, assuming far-field omni-directional transmit antennas, and $d$ is the actual link distance between the transmitter and receiver. ${ }^{5}$ In such models, $\alpha$ typically ranges from $2-4$, and is a function of the propagation environment being considered. Free-space propagation normally tends to have values of $\alpha$ close to 2 , in contrast to dense urban areas, which commonly have values closer to $4 .{ }^{6}$ In this thesis, $\alpha$ values are selected from above range, depending on the propagation environment being modeled in a particular chapter. Moreover, the

\footnotetext{
${ }^{4}$ Note that this model is also known as the simplified pathloss model [15].

${ }^{5}$ Note that in many studies, the constant $v$ also includes the relevant transmit and receive antenna gains.

${ }^{6}$ It is worth noting that in LoS indoor environments, attenuation exponents of less than 2 are also observed in some measurement campaigns, due to the waveguiding effects in the propagation channel. For a further discussion, the interested reader is refereed to [35].
} 
effects of large obstacles such as buildings, in the propagation paths between the transmitter and the receiver are often statistically modeled as a log-normal random variable $[14,15,27,30]$, having the following representation:

$$
\zeta=10^{(S / 10)},
$$

where $S \sim \mathcal{N}\left(0, \sigma_{\text {sf }}^{2}\right)$ and $\sigma_{\text {sf }}$ is the standard deviation of shadow-fading in $\mathrm{dB}$. Similar to geometric attenuation, the standard deviation of shadow-fading depends on the propagation environment and the relative terrain present in the cellular link. Typically, values from 3-8 dB are quoted in the literature, for rural, suburban, and urban environments $[14,15,30,36]$. In this thesis, standard deviation values from the above range are considered, depending on the propagation environment assumed for a particular chapter. As a result of the above discussion, the combined effect of geometric attenuation and shadow-fading on the link gain can be modeled as

$$
\beta=v\left(\frac{d_{0}}{d}\right)^{\alpha} \zeta
$$

In general, throughout the thesis, it is assumed that the link gain is assumed to be known at the transmitter. Assuming block fading, the radio channel is often modeled as a snapshot of a real link between a transmitter and a receiver, in a short time duration. This small transmission duration is often called a coherence interval. ${ }^{7}$ The power variation due to large-scale fading over many coherence intervals is negligible, which justifies the deterministic assumption of large-scale fading. Typically, it is believed that large-scale fading co-efficients change approximately 40 times less frequently than the instantaneous smallscale fading, which changes rapidly within the duration of the coherence time [37]. As a result of this, small-scale fading is treated in general (and thus in the thesis) as a random phenomena that greatly influences the design of efficient digital cellular systems.

\footnotetext{
${ }^{7}$ Coherence time is the time duration over which the channel impulse response is considered to be not varying [15].
} 
This leads to the discussion in the following section.

\subsubsection{Small-Scale Fading}

\section{Uncorrelated I.I.D. Rayleigh Fading}

An uncorrelated, independent and identically distributed (i.i.d.) Rayleigh fading model is commonly used to describe the power level of the received signal, assuming there is rich scattering in the propagation channel $[14,36]$. This is most commonly found in dense urban environments, where no LoS exists between the transmitter and the receiver, with a large number of scattered directions creating replicas of the transmitted signal from each direction to the receive antenna. This classical small-scale fading channel model is used as a baseline case throughout the thesis for performance evaluation of the considered digital cellular systems. With Rayleigh fading, the fading amplitude of the channel, denoted by $X$, is distributed according to

$$
f_{X}(x)=2 x e^{-x^{2}}, x \geq 0
$$

where $\mathbb{E}\left[X^{2}\right]=1$. Next, modeling of a dominant LoS direction in addition to a large number of scattered directions is considered.

\section{Uncorrelated Ricean Fading}

A Ricean fading model is employed typically when describing scenarios where a LoS direction is present in the propagation channel $[14,15,36]$. With Ricean fading, the channel fading amplitude, $X$ is distributed according to the probability density

$$
f_{X}(x)=\frac{2 x}{Y} e^{-\frac{x^{2}}{Y}-\vartheta^{2}} I_{0}\left(\frac{2 x^{2}}{Y} x\right), x \geq 0
$$

where $Y$ and $\vartheta^{2}$ are the power of the scattered and specular (LoS) paths, respectively. Furthermore, $I_{0}(\cdot)$ is the zeroth-order Bessel function of the first-kind. Unlike Rayleigh, an important parameter in the Ricean probability density is 
Transmitter

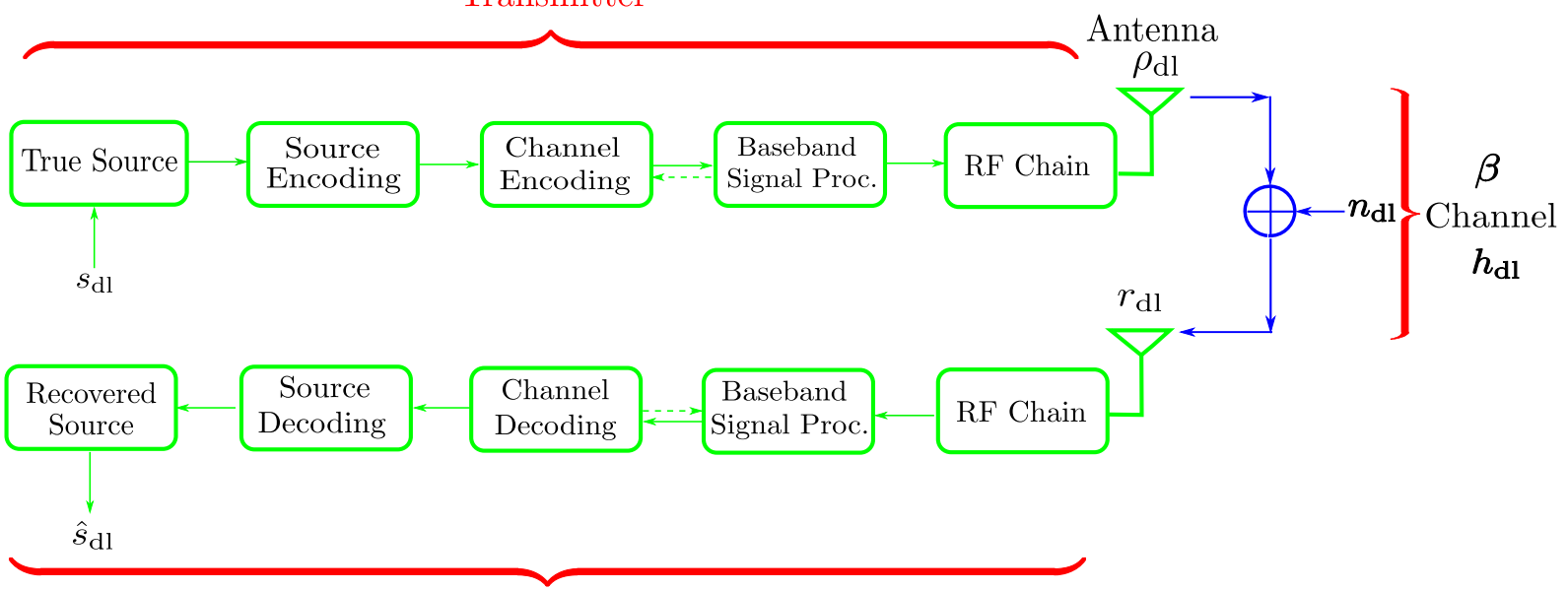

Receiver

Figure 2.1: A digital SISO system.

the Ricean $K$-factor (also known as the Rice factor), which defines the ratio of the powers in the LoS to the scattered components, and is given by

$$
K=\frac{\vartheta^{2}}{Y}
$$

Considering this, $\mathbb{E}\left[X^{2}\right]=Y+\vartheta^{2}$. Usually, the average power in the Ricean amplitude is normalized to unity. This results in $Y+\vartheta^{2}=1$. This along with the constraint in $(2.6)$ gives $Y=\frac{1}{1+K}$ and $\vartheta^{2}=\frac{K}{K+1}$. In this thesis, the latter representation will be used for performance analysis and evaluation of the considered systems. In the following section, a brief discussion of SISO channels is presented before moving onto MIMO channels. ${ }^{8}$

\section{$2.2 \quad$ SISO Channels}

A block diagram of a typical SISO system is shown in Fig. 2.1. The figure considers a scenario where the source transmits a stream of data through the entire signal processing chain, across a frequency-flat fading channel. ${ }^{9}$ If the trans-

\footnotetext{
${ }^{8}$ The section on SISO channels is included for completeness only, and is not further utilized in the thesis.

${ }^{9}$ Later in the thesis, as the focus is primarily on the analysis of various current and future cellular systems, the work presented only considers frequency-flat fading channels, where the
} 
mitter and the receiver are assumed to be perfectly synchronized, the received signal in the $t$-th time instance, in complex baseband representation, is given by

$$
r_{\mathrm{dl}}(t)=\rho_{\mathrm{dl}}\left[g_{\mathrm{dl}}(t) s_{\mathrm{dl}}(t)\right]+n_{\mathrm{dl}}(t)=\rho_{\mathrm{dl}} \beta^{\frac{1}{2}} h_{\mathrm{dl}}(t) s_{\mathrm{dl}}(t)+n_{\mathrm{dl}}(t) .
$$

Here, $\rho_{\mathrm{dl}}$ is the average transmit power available for transmission, $s_{\mathrm{dl}}$ is the transmitted complex data signal, $r_{\mathrm{dl}}$ is the complex received signal, $n_{\mathrm{dl}}$ is the zero mean, variance $\sigma_{\mathrm{dl}}^{2}$ complex Gaussian noise, i.e., $n_{\mathrm{dl}} \sim \mathcal{C N}\left(0, \sigma_{\mathrm{dl}}^{2}\right)$, and $g_{\mathrm{dl}}=\beta^{\frac{1}{2}} h_{\mathrm{dl}}(t)$ is the complex channel response at time $t .^{10}$ Note that $h_{\mathrm{dl}}$ contains the effects of fading induced by the propagation in the channel. In Fig. 2.1, $\beta$ denotes the link gain. From the previous discussion, it can be seen that both the large-scale and small-scale fading contribute to the instantaneous value of the received power at time $t$. Since the large-scale fading co-efficient is assumed to be a deterministic value in the thesis, the overall channel response can be decomposed into a product of large and small-scale fading, respectively.

Under i.i.d. Rayleigh fading, $h_{\mathrm{dl}}$ is a complex Gaussian random variable with zero-mean and unit-variance, such that $\mathbb{E}\left[\left|h_{\mathrm{dl}}\right|^{2}\right]=1$. That is, $h_{\mathrm{dl}}(t) \sim$ $\mathcal{C N}(0,1)$. In contrast to this, under Ricean fading, $h_{\mathrm{dl}}(t) \sim \mathcal{C N}\left(\sqrt{\frac{K}{K+1}} A, \frac{1}{1+K}\right)$, where $\sqrt{\frac{K}{K+1}} A$ is the complex mean accounting for the power of the specular path. For the normalized Ricean fading channel, $|A|^{2}=1$. Hence, $\mathbb{E}\left[\left|h_{\mathrm{dl}}(t)\right|^{2}\right]=$ 1 , giving rise to $\mathbb{E}\left[|g(t)|^{2}\right]=\beta$. Therefore, the power in the specular path from the transmitter to the receiver is given by $\frac{\beta K}{K+1}$. The above discussion is now generalized to MIMO channels, where the statistical channel models are adapted.

Without loss of generality, for the remainder of the thesis, the time index $t$ is omitted for the ease of notation and only one time instance is considered. The following section presents the necessary background on point-to-point MIMO channels.

transfer function of the channel is essentially constant over the signal bandwidth.

${ }^{10}$ Note that the subscript dl in equation (2.7) is used to denote the down (forward) link from the transmitter to the receiver. 


\subsection{MIMO-OFDM Channels}

In this section, point-to-point MIMO channels [5-7, 14] are discussed in a frequencyflat fading environment. The use of multiple antennas at either end of the link enables transmission of parallel data streams which are spatially multiplexed. A downlink MIMO architecture with $M$ transmit and $L$ receive antennas is considered, which can be mathematically expressed at time $t$ as

$$
\boldsymbol{r}_{\mathrm{dl}}=\rho_{\mathrm{dl}} \boldsymbol{G}_{\mathrm{dl}}^{\mathrm{H}} \boldsymbol{s}_{\mathrm{dl}}+\boldsymbol{n}_{\mathrm{dl}}=\rho_{\mathrm{dl}} \boldsymbol{D}^{\frac{1}{2}} \boldsymbol{H}_{\mathrm{dl}}^{\mathrm{H}} \boldsymbol{s}_{\mathrm{dl}}+\boldsymbol{n}_{\mathrm{dl}}
$$

where $\boldsymbol{r}_{\mathrm{dl}}$ is an $L \times 1$ vector of received signal observations at the $L$ receive antennas at time $t, \boldsymbol{s}_{\mathrm{dl}}$ is the $M \times 1$ data symbols transmitted by the $M$ antennas at time instance $t, \boldsymbol{n}_{\mathrm{dl}}$ is the $L \times 1$ vector of additive Gaussian noise entries, such that $\boldsymbol{n}_{\mathrm{dl}} \sim \mathcal{C} \mathcal{N}\left(0, \sigma_{\mathrm{dl}}^{2} \boldsymbol{I}_{L}\right)$ with noise power $\sigma_{\mathrm{dl}}^{2}$. Furthermore, $\boldsymbol{H}_{\mathrm{dl}}^{\mathrm{H}}$ is the $L \times M$ MIMO channel matrix containing complex co-efficients from each transmit to each receive antennas, and $\boldsymbol{D}$ is a $L \times L$ matrix of link gains from each transmit to receive antenna. Orthogonal frequency-division multiplexing (OFDM) waveforms across time and frequency are assumed, with narrowband signaling within each OFDM subcarrier. ${ }^{11}$ For ease of notation, the subcarrier index is omitted for simplicity. ${ }^{12}$ Similar to the SISO case, a block fading model is assumed where the large-scale fading (link gain) co-efficients stay constant during the channel coherence interval. ${ }^{13}$ The small-scale fading channel is independent in different coherence intervals. The fundamental blocks of a fully digital MIMO architecture are demonstrated in Fig. 2.2. Here, the additional RF chains and antennas required are explicitly shown, as they have a strong influence on the performance of MIMO systems.

\footnotetext{
${ }^{11}$ This is assumed in today's fourth generation (4G) Long Term Evolution-Advanced (LTEA) systems.

${ }^{12}$ Note that in reality, it is important to note that the small-scale fading co-efficients from each transmit to receive antennas depend on the antenna index and on OFDM subcarrier index. For the remainder of the thesis, the author assumes that OFDM waveforms will be used for all systems studied.

${ }^{13}$ It is convinent to measure the length of the coherence interval in terms of the number of OFDM symbols that can be transmitted within that interval.
} 


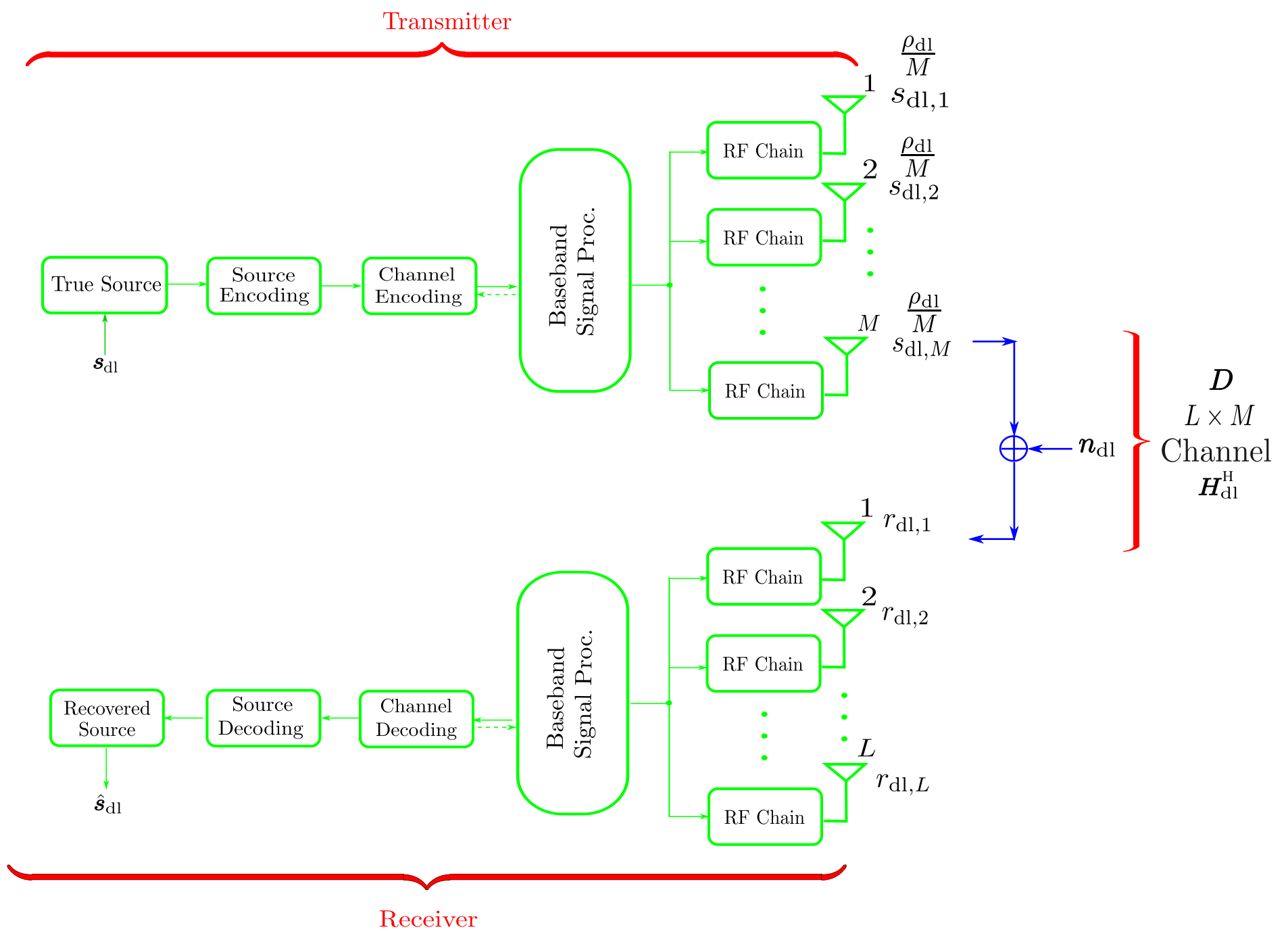

Figure 2.2: A fully digital MIMO communication system. 
Remark 2.3.1. Note that no power control is assumed and thus the total transmit power is equally divided by $M$, such that each antenna receives a net power of $\rho_{\mathrm{dl}} / M$. With multiple antennas at either end of the link, a MIMO system gives increased spectral efficiencies in comparison to a SISO system. The spectral efficiency gain can be characterized by its multiplexing gain, defined as $[5,38]$

$$
\lim _{\mathrm{SNR} \rightarrow \infty}\left\{\frac{\mathrm{R}_{\mathrm{dl}}(\mathrm{SNR})}{\log _{2}(\mathrm{SNR})}\right\}=s
$$

where $\mathrm{R}_{\mathrm{dl}}(\mathrm{SNR})$ denotes the downlink spectral efficiency of a MIMO link given a fixed link signal-to-noise-ratio (SNR). ${ }^{14}$ The link SNR is defined as the ratio of the transmit power, $\rho_{\mathrm{dl}}$, to noise power at the $l$-receive antenna, $\sigma_{l, \mathrm{dl}}^{2}$. Note that $\sigma_{\mathrm{dl}, 1}^{2}=, \ldots, \sigma_{\mathrm{dl}, L}^{2}{ }^{15}$ The multiplexing gain characterizes the maximum number of spatially usable degrees of freedom provided by the MIMO channel $[5,38]$.

Remark 2.3.2. On the other hand, the deployment of multiple antenna elements reduces the average bit error rate of a digital communication system for a fixed number of downlink data streams. For instance, transmitting waveforms with identical information exploits the additional spatial diversity in the communication channel, provided by multiple antennas. Diversity is known as the most effective strategy to combat the effects of fading $[5,14,15,38]$. This diversity gain is often characterized as

$$
\lim _{\mathrm{SNR} \rightarrow \infty}\left\{\frac{\log _{2} P_{e}(\mathrm{SNR})}{\log _{2}(\mathrm{SNR})}\right\}=-d
$$

where $P_{e}(\mathrm{SNR})$ denotes the average probability of error at a fixed link SNR. It is worth noting that there exists a trade-off between diversity and the multiplexing gain of a MIMO system, since the added spatial paths can be employed for enhancing the link reliability, or the spectral efficiency. For a further discussion on the spatial multiplexing to diversity trade-off, the interested reader is referred to $[5,14,15,38]$. Note that $s$ and $d$ are defined in equations (2.9) and (2.10), respectively.

\footnotetext{
${ }^{14}$ The spectral efficiency of a point-to-point MIMO link is described in Section 2.3.4.

${ }^{15}$ This holds for the remainder of the chapter.
} 
Remark 2.3.3. As the performance of MIMO systems ultimately depend on the propagation channels they operate in, the following section outlines some typical statistical models which are used to predict the performance of MIMO systems. The presented channel models will feature extensively throughout the thesis, and will help to develop the novel random matrix theory results. For ease of exposition, the discussion in the rest of the chapter considers the normalized small-scale fading channel in $\boldsymbol{H}_{\mathrm{dl}}^{\mathrm{H}}$, which excludes the effects of the link gains.

\subsubsection{Uncorrelated I.I.D. Rayleigh Fading}

As for SISO channels, the Rayleigh fading assumption is often used by cellular engineers as it allows for modeling of very general propagation scenarios which are rich in scattering. It corresponds to modeling the narrowband transmission between a pair of transmit and receive antennas via a sum of a large number of contributions, each with random and statistically independent phases, DoDs and DoAs [39]. Due to this reason, each individual channel is still a zero-mean, unit-variance complex Gaussian random variable. That is, the channel from transmit antenna $m$ to receive antenna $l$ in $h_{l, m} \sim \mathcal{C N}(0,1)$. As such, the composite MIMO channel from each transmit to each receive antennas can be modeled as

$$
\boldsymbol{H}_{\mathrm{dl}}^{\mathrm{H}} \sim \mathcal{C N}\left(\mathbf{0}, \boldsymbol{I}_{M}\right)
$$

where $\boldsymbol{I}_{M}$ is a $M \times M$ identity matrix.

Remark 2.3.4. The assumption of uncorrelated scattering in (2.11) is reasonable when the inter-element spacing between two antennas, and/or the angular spreading of the electromagnetic radiation at both sides of the link are large enough. ${ }^{16}$ Here, minimal correlation in the spatial domain is induced, and can be assumed to be negligible. ${ }^{17}$ Nevertheless, real world MIMO channels often significantly deviate from such idealistic assumptions due to the reasons listed

\footnotetext{
${ }^{16}$ Note that only the azimuth domain is considered in the thesis. From now on, angular spreading will be referred to as the angular spread for the remainder of the thesis.

${ }^{17}$ The concept of correlation over the spatial domain (spatial correlation) is further explained in Section 2.3.2 in further detail.
} 
below:

Remark 2.3.5. (1) Limited angular spread and/or reduced array sizes cause the channels to become more spatially correlated, i.e., the channels lose their independence. (2) The specular contribution may induce the channel statistics to become Ricean. ${ }^{18}$ As a result, the following section studies the behavior of semi-correlated Rayleigh fading channels with spatial correlation at the transmit end of the link for a downlink system and at the receive end of the link for an uplink system, respectively.

\subsubsection{Semi-Correlated Rayleigh Fading}

Due to insufficient spacing between the transmit antenna elements and/or the lack of scattering in the propagation channel, signals impinging on different antennas in an array will often be correlated over a given space. Spatial correlation depends on various physical parameters of the antenna array, and the scatterer characteristics [14, 40-44].

Remark 2.3.6. The spatial correlation between adjacent antenna elements is mainly governed by three parameters [21]: (1) the distances between antennas [45], (2) the angular spread of the arrival incident waves, and (3) the central DoA of the incident waves. To model a Rayleigh fading channel with spatial correlation at the transmitter, the MIMO channel matrix can be written as [14]

$$
\boldsymbol{H}_{\mathrm{dl}}^{\mathrm{H}}=\boldsymbol{H}_{\mathrm{i} . \mathrm{i} . \mathrm{d}, \mathrm{dl}}^{\mathrm{H}} \boldsymbol{R}^{\frac{1}{2}},
$$

where $\boldsymbol{H}_{\mathrm{i} . \mathrm{i} . \mathrm{d} . \text {,dl }}^{\mathrm{H}}$ is as defined in (2.11), and $\boldsymbol{R}$ is the $M \times M$ transmit spatial correlation matrix. The spatial correlation co-efficient between two transmit antennas $l$ and $k \in 1, \ldots, M$, is defined as [19]

$$
\bar{R}_{l, k}=\frac{\mathbb{E}\left[\boldsymbol{h}_{\mathrm{dl}, l}^{\mathrm{H}} \boldsymbol{h}_{\mathrm{dl}, k}\right]}{\sqrt{\mathbb{E}\left[\boldsymbol{h}_{\mathrm{dl}, l}^{\mathrm{H}} \boldsymbol{h}_{\mathrm{dl}, l}\right] \mathbb{E}\left[\boldsymbol{h}_{\mathrm{dl}, k}^{\mathrm{H}} \boldsymbol{h}_{\mathrm{dl}, k}\right]}},
$$

where $\boldsymbol{h}_{\mathrm{dl}, l}^{\mathrm{H}}$ is a $1 \times M$ vector denoting the small-scale fading co-efficients from all

\footnotetext{
${ }^{18}$ Note that Ricean channels are covered in Section 2.3.3.
} 
$M$ transmit antenna to the $l$-th receive antenna. From the definition in (2.13), one can note that spatial correlation is defined over an ensamble of elementto-element propagation channel vectors. For the case where this ensamble is composed of all channel vectors in the entire cell (coverage area of interest), the resulting correlation matrix is fixed (equal) for all terminals. Nevertheless, in this thesis, a more general propagation environment is considered, where the physical channel effects such as antenna spacing and angular spread of incident propagation are invoked to motivate unequal correlation matrices. This is particularly applicable to multiuser systems, as shown in Chapters 4-8 of the thesis, where the ensamble is restricted to the set of all possible locations where the correlation patterns may be extremely similar. Given a fixed set of inter-element spacings, this is due to the similarities in the angular spread characteristics. The variation of the angular spread is further described in Section 7.4.2, where a restricted set of ensamble is employed to physically represent spatial correlation.

Remark 2.3.7. Note that $\left|\bar{R}_{l, k}\right| \leq 1$, with equality when the two antenna element channels are fully correlated, i.e., fully dependent on each other. In contrast to this, when $\left|\bar{R}_{l, k}\right|=0$, the two channels are fully independent. Generally, spatial correlation can be reduced by altering the array geometry, such that adjacent elements are sufficiently de-correlated. To this end, a uniform linear array (ULA) located on the $x-y$ plane is considered throughout the thesis. The antenna geometry is as demonstrated in Fig. 2.3. Here, $M$ is the total number of antenna elements in the ULA and the inter-element spacing is denoted by $d_{s}$ meters.

Often, the resultant expressions in (2.13) are too complex to perform for any meaningful statistical analysis. As a result, a number of simplified spatial correlation models have been developed and proposed. A simple and commonly employed spatial correlation model, used for performance evaluation throughout the thesis is known as the exponential correlation model [46] and is described in the following subsection. It is worth noting that later in the thesis, in Chapter 7, the performance of a broad range of spatial correlation models is compared and their impact on the spectral efficiency of multiuser MIMO systems is char- 


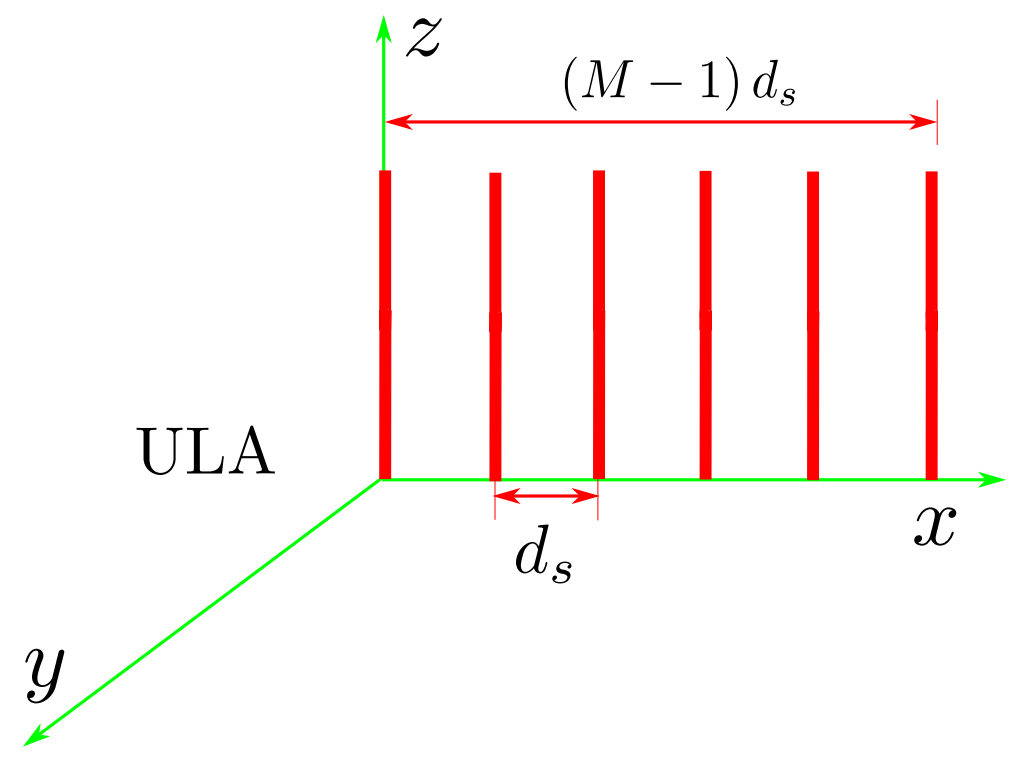

Figure 2.3: ULA geometry on the $x$-axis with $M$ antenna elements, each with an inter-element spacing of $d_{s}$.

acterized.

\section{Exponential Spatial Correlation Model}

The most common and simple correlation model was proposed in [46]. The model was proposed by fitting an exponential function to the non-monotonically decaying peaks of the Jakes spatial correlation model, which assumes a uniform ring of scatterers around the transmitter (see Chapter 7, Section 7.2 for a further discussion) $[47,48]$. It is a rather simplified model, as it crudely approximates the true correlation in the channels via a single parameter, $\varphi$. However, the advantage of this model over many others lies in its simplicity, as the correlation magnitude is controlled with a single parameter. This makes the model useful for consideration in analytical studies. Using such a model, the spatial correlation 
matrix, $\boldsymbol{R}$, can be written as

$$
\boldsymbol{R}=\left[\begin{array}{cccc}
1 & \varphi & \ldots & \varphi^{M-1} \\
(\varphi)^{*} & 1 & \ldots & \varphi^{M-2} \\
\vdots & \vdots & \ddots & \vdots \\
\left(\varphi^{M-1}\right)^{*} & \left(\varphi^{M-2}\right)^{*} & \ldots & 1
\end{array}\right]
$$

where $\varphi$ is the exponential decay parameter, with $|\varphi| \leq 1$.

Remark 2.3.8. Naturally, a large value of $|\varphi|$ indicates higher spatial correlation in comparison to a smaller value of $|\varphi|$. From this, one can relate $|\varphi|$ to the inter-element spacing, $d_{s}$, at the ULA with an inversely proportional relationship. However, the model tends to neglect the relationship between the spatial correlation induced with closer inter-element spacings, illuminating a finite amount of angular spread within the vicinity of the terminal, controlled by the degree of local scattering around the terminal. Further details on the comparison of the exponential model with other more complex models, such as the remote scattering models can be found in Chapter 7 of the thesis.

While the representation of (2.12) is most general for semi-correlated Rayleigh fading channels, some channels are likely to have dominant LoS directions. In such cases, the use of a Ricean fading model is most commonly employed. This is the focus of the discussion in the following section.

\subsubsection{Uncorrelated Ricean Fading}

There often exists situations in wireless systems where there may be a strong LoS component in the propagation channel. Statistically, this leads to a Ricean distribution of the resulting envelope fading. Typically, Ricean channels are a mixture of scattered and specular (LoS) components, denoted by the $L \times$ $M$ matrices, $\widetilde{\boldsymbol{H}}_{\mathrm{dl}}^{\mathrm{H}}$ and $\overline{\boldsymbol{H}}_{\mathrm{dl}}^{\mathrm{H}}$. The matrix $\overline{\boldsymbol{H}}_{\mathrm{dl}}^{\mathrm{H}}$, corresponding to the specular 
components, has fixed phase-shift only entries given by [36, 49]

$$
\overline{\boldsymbol{H}}_{\mathrm{dl}}^{\mathrm{H}}=\left[\begin{array}{cccc}
e^{j \bar{\phi}_{1,1}} & e^{j \bar{\phi}_{1,2}} & \ldots & e^{j \bar{\phi}_{1, M}} \\
e^{j \bar{\phi}_{2,1}} & e^{j \bar{\phi}_{2,2}} & \ldots & e^{j \bar{\phi}_{2, M}} \\
\vdots & \vdots & \ddots & \vdots \\
e^{j \bar{\phi}_{L, 1}} & e^{j \bar{\phi}_{L, 2}} & \ldots & e^{j \bar{\phi}_{L, M}}
\end{array}\right]
$$

Remark 2.3.9. Note that the value of the phase-shifts are strongly related to the antenna array configurations and its orientation with respect to the direction of the dominant specular component(s). Moreover, when there is a single dominant specular component, $\overline{\boldsymbol{H}}_{\mathrm{dl}}^{\mathrm{H}}$ is likely to be poorly conditioned, thereby likely to decrease the multiplexing gain for a given link SNR. Given a single dominant specular direction with a particular DoD and DoA,

$$
\overline{\boldsymbol{H}}_{\mathrm{dl}}^{\mathrm{H}}=\boldsymbol{a}_{\mathrm{rx}}\left(\bar{\phi}_{\mathrm{rx}, 1}\right) \boldsymbol{a}_{\mathrm{tx}}^{\mathrm{T}}\left(\bar{\phi}_{\mathrm{tx}, \mathrm{l}}\right)
$$

if a sufficiently large separation between the transmit and receive antennas is assumed [36, 49]. ${ }^{19} \operatorname{In}(2.16), \boldsymbol{a}_{\mathrm{rx}}\left(\bar{\phi}_{\mathrm{rx}, l}\right)$ and $\boldsymbol{a}_{\mathrm{tx}}^{\mathrm{T}}\left(\bar{\phi}_{\mathrm{tx}, l}\right)$ are the $L \times 1$ and $1 \times M$ farfield specular array responses of the receive and transmit antenna arrays, respectively. In particular for a ULA, $\boldsymbol{a}_{\mathrm{rx}}\left(\bar{\phi}_{\mathrm{rx}, l}\right)=\left[1, e^{j 2 \pi \bar{d}_{s} \cos \left(\bar{\phi}_{\mathrm{rx}, l}\right)}, \ldots, e^{j 2 \pi \bar{d}_{s}(L-1) \cos \left(\bar{\phi}_{\mathrm{rx}, l}\right)}\right]$, where $\bar{\phi}_{\mathrm{rx}, l}$ is the DoA and $\bar{d}_{s}$ is the inter-element spacing normalized by the carrier wavelength. Likewise, for $\boldsymbol{a}_{\mathrm{tx}}^{\mathrm{T}}\left(\bar{\phi}_{\mathrm{tx}, l}\right)$, the response has an identical structure to $\boldsymbol{a}_{\mathrm{rx}}\left(\bar{\phi}_{\mathrm{rx}, l}\right)$, with a DoA $\bar{\phi}_{\mathrm{tx}, l}$, for a $M$ element ULA. The above situation features in the later chapters of the thesis, such as in Chapters 5 and 8, respectively.

Remark 2.3.10. The expression in (2.16) allows one to observe the potential rank decrease brought by Ricean fading. For instance, considering broadside arrays (when $\bar{\phi}_{\mathrm{tx}, l}=90^{\circ}$ and $\bar{\phi}_{\mathrm{rx}, l}=90^{\circ}$ ), $\overline{\boldsymbol{H}}_{\mathrm{dl}}^{\mathrm{H}}=\mathbf{1}_{L \times M}$. This leads to completely correlated channels with no spatial diversity yielding the rank reduction.

Combining $\overline{\boldsymbol{H}}_{\mathrm{dl}}^{\mathrm{H}}$ with the scattered components in $\widetilde{\boldsymbol{H}}_{\mathrm{dl}}^{\mathrm{H}}$, which have zeromean, unit variance, complex Gaussian entries allows one to write the composite

\footnotetext{
${ }^{19}$ Note that the term "sufficiently large" refers to the separation being greater than the angular Rayleigh resolution of the ULA at both the transmit and receive ends of the link [9].
} 
Ricean channel matrix as

$$
\boldsymbol{H}_{\mathrm{dl}}^{\mathrm{H}}=\sqrt{\frac{K}{K+1}} \overline{\boldsymbol{H}}_{\mathrm{dl}}^{\mathrm{H}}+\sqrt{\frac{1}{1+K}} \widetilde{\boldsymbol{H}}_{\mathrm{dl}}^{\mathrm{H}}
$$

Note that $\mathbb{E}\left[\boldsymbol{H}_{\mathrm{dl}}^{\mathrm{H}}\right]=\sqrt{\frac{K}{K+1}} \overline{\boldsymbol{H}}_{\mathrm{dl}}^{\mathrm{H}}$, and $K$ is the Rice factor, as introduced for the SISO case earlier in Section 2.1.3. Upon characterizing the point-to-point MIMO statistical channel models used throughout the thesis, the ergodic spectral efficiency of MIMO channels is now presented, allowing the author to motivate the use of the eigenvalue distributions of the instantaneous channel correlation matrices. This is the focus of the following subsection.

\subsubsection{Ergodic Spectral Efficiency}

Excluding the link gains, with knowledge of the instantaneous channel state information at the receiver, and assuming equal power allocation across each stream, the downlink ergodic spectral efficiency of a MIMO channel is given by $[5,14,15,36]$

$$
\mathrm{R}_{\mathrm{dl}}=\mathbb{E}\left[\log _{2}\left(\operatorname{det}\left(\boldsymbol{I}_{L}+\frac{\mathrm{SNR}}{M} \boldsymbol{H}_{\mathrm{dl}}^{\mathrm{H}} \boldsymbol{H}_{\mathrm{dl}}\right)\right)\right]
$$

The landmark work of Telatar in [6] showed that the MIMO spectral efficiency can be re-written in terms of the eigenvalues of $\boldsymbol{H}_{\mathrm{dl}}^{\mathrm{H}} \boldsymbol{H}_{\mathrm{dl}}$ as

$$
\begin{aligned}
\mathrm{R}_{\mathrm{dl}} & =\mathbb{E}\left[\sum_{l=1}^{m} \log _{2}\left(1+\frac{\mathrm{SNR}}{M} \lambda_{l}\right)\right] \\
& =\sum_{l=1}^{m} \mathbb{E}\left[\log _{2}\left(1+\frac{\mathrm{SNR}}{M} \lambda_{l}\right)\right] \\
& \stackrel{(a)}{=} m\left\{\mathbb{E}\left[\log _{2}\left(1+\frac{\mathrm{SNR}}{M} \lambda_{\mathrm{arb}}\right)\right]\right\} \\
& \stackrel{(b)}{=} \int_{0}^{\infty} \log _{2}\left(1+\frac{\mathrm{SNR}}{M} \lambda_{\text {arb }}\right) f_{0}\left(\lambda_{\mathrm{arb}}\right) d \lambda_{\mathrm{arb}},
\end{aligned}
$$

where $\lambda_{l}$ in (2.20) is the $l$-th eigenvalue of $\boldsymbol{H}_{\mathrm{dl}}^{\mathrm{H}} \boldsymbol{H}_{\mathrm{dl}}$. Moreover, $(a)$ is obtained rewriting (2.19) with the arbitrary eigenvalue, $\lambda_{\text {arb }} \in\left\{\lambda_{1}, \ldots, \lambda_{m}\right\}$. Note that from now until the remainder of the chapter, $m=\min (M, L)$ and $n=\max (M, L)$. 
In addition to the above, $(b)$ is obtained by performing the expectation with respect to the arbitrary eigenvalue density.

Remark 2.3.11. The formulation in (2.21) is usually preferred as it only involves the arbitrary eigenvalue of $\boldsymbol{H}_{\mathrm{dl}}^{\mathrm{H}} \boldsymbol{H}_{\mathrm{dl}}$, the channel correlation matrix. In contrast, (2.21) is awkward because these eigenvalues have unique probability density functions, which makes analysis of performance metrics such as the ergodic spectral efficiency more difficult. Hence, knowledge of such arbitrary densities for a given (statistical) propagation channel allows one to predict the ergodic spectral efficiency of MIMO systems. For the channels of interest to the thesis, $\boldsymbol{H}_{\mathrm{dl}}^{\mathrm{H}} \boldsymbol{H}_{\mathrm{dl}}$ takes on the form of a Wishart random matrix [50-54]. In particular, when $\boldsymbol{H}_{\mathrm{dl}}^{\mathrm{H}}$ undergoes Rayleigh and semi-correlated Rayleigh fading, the $\boldsymbol{H}_{\mathrm{dl}}^{\mathrm{H}} \boldsymbol{H}_{\mathrm{dl}}$ follows uncorrelated central and correlated central Wishart structure. In the case when $\boldsymbol{H}_{\mathrm{dl}}^{\mathrm{H}}$ undergoes uncorrelated Ricean fading, $\boldsymbol{H}_{\mathrm{dl}}^{\mathrm{H}} \boldsymbol{H}_{\mathrm{dl}}$ has a non-central, uncorrelated Wishart distribution [50-54].

Remark 2.3.12. In addition to the arbitrary densities, the eigenvalue density of an arbitrary pair of eigenvalues drawn from the channel correlation matrix is also vital for the analysis of MIMO systems. Using such densities, researchers have been able to analyze the variance of MIMO spectral efficiency (see e.g., $[55,56])$. Furthermore, as will be seen in Chapters 4 and 5 , for multiuser MIMO systems, knowledge of such densities also allows one to analyze the expected signal-to-interference-plus-noise-ratio (SINR) with multiple types of transmit precoding approaches such as zero-forcing (ZF) and regularized zero-forcing precoding. Both the arbitrary and the joint arbitrary pair of eigenvalue densities can also be used to predict the instantaneous ZF SNR at a particular receive antenna, as demonstrated in Chapter 5. Further to the above, with growing interest in large MIMO systems, convergence of the eigenvalue densities, as $M \rightarrow \infty$ with $L$ fixed helps one to get an intuitive understanding of the required system dimensions before fast-fading and uncorrelated noise can vanish, reaching favorable propagation conditions [9,57-61]. This will be demonstrated in Chapter 8 of the thesis with fixed sized ULAs.

With such a direct dependence on the performance analysis of MIMO sys- 
tems, eigenvalue densities of Wishart matrices are a necessity to facilitate analytical progress. A broad overview of most other applications of eigenvalue densities to MIMO systems is given in [62], as well as references therein. Despite the advances in random matrix theory, tractable expressions for the joint arbitrary eigenvalue densities of the correlated central and uncorrelated non-central Wishart matrices remain a challenge. These are derived in the following section of the thesis, to facilitate further analysis. As the further analysis also relies on the knowledge of the arbitrary eigenvalue densities, a comprehensive overview of uncorrelated and correlated central, as well as uncorrelated non-central Wishart eigenvalue densities is presented.

\subsection{Eigenvalue Densities of Wishart Matrices}

\subsubsection{Uncorrelated Central Wishart Matrices}

Definition 2.4.1. When $\boldsymbol{H}_{\mathrm{dl}}^{\mathrm{H}}$ undergoes uncorrelated i.i.d. Rayleigh fading, $\boldsymbol{H}_{\mathrm{dl}}^{\mathrm{H}} \boldsymbol{H}_{\mathrm{dl}}$ follows an uncorrelated complex central Wishart structure with $m$ degrees of freedom (d.o.f.) having a covariance matrix $\boldsymbol{I}_{m}$. Let $\lambda_{\text {arb }}$ denote an arbitrary eigenvalue of $\boldsymbol{H}_{\mathrm{dl}}^{\mathrm{H}} \boldsymbol{H}_{\mathrm{dl}}$ drawn from $\lambda_{1}, \ldots, \lambda_{m}$. Then, the density of $\lambda_{\text {arb}}$, denoted by $f_{0}$, is then given by [6]

$$
f_{0}\left(\lambda_{\mathrm{arb}}\right)=m^{-1} \sum_{i=1}^{m} \frac{(i-1)}{(i-1+n-m)} \lambda_{\text {arb }}^{n-m} e^{-\lambda_{\text {arb }}} \kappa_{i-1}^{(n-m)}\left(\lambda_{\text {arb }}\right)^{2},
$$

where $\kappa_{i-1}^{(n-m)}$ is a generalized Laguerre polynomial of order $i-1$, defined as [63]

$$
\kappa_{i-1}^{(n-m)}\left(\lambda_{\mathrm{arb}}\right)=\sum_{s=0}^{i-1}(-1)^{s}\left(\begin{array}{c}
i+1+n-m \\
i-1-s
\end{array}\right) \frac{\lambda_{\mathrm{arb}}^{s}}{s !} .
$$

Definition 2.4.2. Under the same conditions as Definition 2.4.1., let $\left(\lambda_{1}, \lambda_{2}\right)$ be any two arbitrary, unordered eigenvalues of $\boldsymbol{H}_{\mathrm{dl}}^{\mathrm{H}} \boldsymbol{H}_{\mathrm{dl}}$. The joint density of such 
a pair of arbitrary eigenvalues is denoted by $f_{0}\left(\lambda_{1}, \lambda_{2}\right)$ and is given by [55]

$$
\begin{aligned}
f_{0}\left(\lambda_{1}, \lambda_{2}\right) & =(m(m-1))^{-1} \sum_{i=1}^{m} \sum_{\substack{j=1 \\
j \neq i}}^{m}\left(\lambda_{1} \lambda_{2}\right)^{n-m} e^{-\left(\lambda_{1}+\lambda_{2}\right)} \Phi\left[\kappa_{i-1}^{(n-m)}\left(\lambda_{1}\right)^{2} \kappa_{j-1}^{(n-m)}\left(\lambda_{2}\right)^{2}\right. \\
& \left.-\kappa_{i-1}^{(n-m)}\left(\lambda_{1}\right) \kappa_{j-1}^{(n-m)}\left(\lambda_{1}\right) \kappa_{i-1}^{(n-m)}\left(\lambda_{2}\right) \kappa_{j-1}^{(n-m)}\left(\lambda_{2}\right)\right]
\end{aligned}
$$

where

$$
\Phi=\frac{(i-1) !(j-1) !}{(i-1+n-m) !(j-1+n-m) !},
$$

and $\kappa_{i-1}^{(n-m)}(\cdot)$, as well as $\kappa_{j-1}^{(n-m)}(\cdot)$ are as defined in $(2.23)$.

Remark 2.4.1. The same set of densities for the correlated complex central Wishart case are provided in the following subsection. In particular, the arbitrary eigenvalue density is a known result from the literature [64] and the joint pair of arbitrary eigenvalue density is derived in the thesis.

\subsubsection{Correlated Central Wishart Matrices}

Definition 2.4.3. When $\boldsymbol{H}_{\mathrm{dl}}^{\mathrm{H}}$ undergoes semi-correlated Rayleigh fading, $\boldsymbol{H}_{\mathrm{dl}}^{\mathrm{H}} \boldsymbol{H}_{\mathrm{dl}}$ follows a correlated complex central Wishart structure with $m$ degrees of freedom and a $M \times M$ covariance matrix $\boldsymbol{R}$. For the case when $L \geq M$, the joint density of $m$ ordered eigenvalues of $\boldsymbol{H}_{\mathrm{dl}}^{\mathrm{H}} \boldsymbol{H}_{\mathrm{dl}}$ of $\lambda_{1} \geq \lambda_{2} \geq, \ldots, \geq \lambda_{m}$ is given by $[64]$

$$
f_{\text {ord }, \mathrm{sc}}\left(\lambda_{1}, \ldots, \lambda_{m}\right)=\frac{\prod_{k=1}^{m} \lambda_{k}^{n-m} \prod_{k<l}^{m}\left(\lambda_{k}-\lambda_{l}\right) \operatorname{det}(\boldsymbol{\Xi})}{\prod_{l=1}^{m}(n-l) ! \operatorname{det}(\boldsymbol{R})^{n} \prod_{k<l}^{m}\left(\frac{1}{\theta_{l}}-\frac{1}{\theta_{k}}\right)},
$$

where the $(i, j)$-th element of $\boldsymbol{\Xi}$ is given by $e^{-\lambda_{i}} / \theta_{j}$ with $\theta_{1}, \ldots, \theta_{m}$ denoting the $m$ eigenvalues of $\boldsymbol{R}$.

Definition 2.4.4. From Definition 2.4.3, the density of an arbitrary eigenvalue, $\lambda_{\text {arb,sc }}$, of the correlated central Wishart matrix can be written as [64]

$$
f_{\mathrm{o}, \mathrm{sc}}\left(\lambda_{\mathrm{arb}, \mathrm{sc}}\right)=\chi \sum_{i=1}^{m} \sum_{\substack{j=1 \\ j \neq i}}^{m} \lambda_{\mathrm{arb}, \mathrm{sc}}^{n-m+j-1} e^{-\lambda_{\mathrm{arb}, \mathrm{sc}} / \theta_{i}} \mathcal{D}(i, j),
$$


where

$$
\chi=\frac{1}{m \prod_{l=1}^{m}(n-l) ! \operatorname{det}(\boldsymbol{R})^{n} \prod_{k<l}^{m}\left(\frac{1}{\theta_{l}}-\frac{1}{\theta_{k}}\right)},
$$

and $\mathcal{D}(i, j)$ is the $(i, j)$-th co-factor of an $m \times m$ matrix whose $(l, k)$-th entry is given by $(n-m+k-1) ! / \theta_{l}^{-n+m-k}$.

Definition 2.4.5. Under the same conditions as Definitions 2.4.3 and 2.4.4, for the case where $L<M$, the joint density of $m$ ordered eigenvalues of $\boldsymbol{H}_{\mathrm{dl}}^{\mathrm{H}} \boldsymbol{H}_{\mathrm{dl}}$, $\lambda_{1} \geq \lambda_{2} \geq, \ldots, \geq \lambda_{m}$, is given by $[65]$

$$
f_{\text {ord }}\left(\lambda_{1}, \ldots, \lambda_{m}\right)=T \sum_{\phi}(-1)^{\operatorname{per}(\phi)} \prod_{i=1}^{m} \lambda_{i}^{\phi_{i}} \operatorname{det}\left(\boldsymbol{\Delta}_{n, m}\right)
$$

where the summation is over all permutations, $\phi$, from $0,1, \ldots, m-1$ and

$$
T=\frac{1}{\prod_{j=1}^{n} j ! \operatorname{det}\left(\boldsymbol{\Delta}_{n}\right)}
$$

with $\boldsymbol{\Delta}_{n}$ defined as the $n \times n$ Vandermonde matrix

$$
\boldsymbol{\Delta}_{n}=\left[\begin{array}{cccc}
1 & \theta_{1} & \ldots & \theta_{1}^{n-1} \\
\vdots & \vdots & \ddots & \vdots \\
1 & \theta_{n} & \ldots & \theta_{n}^{n-1}
\end{array}\right]
$$

Furthermore, $\boldsymbol{\Delta}_{n, m}$ is the $n \times n$ matrix defined as

$$
\boldsymbol{\Delta}_{n, m}=\left[\begin{array}{ccccc}
1 & \ldots & \theta_{1}^{n-m-1} & \theta_{1}^{n-m-1} e^{-\lambda_{1} / \theta_{1}} & \ldots \\
\vdots & \vdots & \vdots & \vdots & \vdots \\
1 & \ldots & \theta_{n}^{n-m-1} & \theta_{n}^{n-m-1} e^{-\lambda_{1} / \theta_{n}} & \ldots
\end{array}\right]
$$

Definition 2.4.6. Under the same conditions as Definitions 2.4.3, 2.4.4 and 
2.4.5, the density of the arbitrary eigenvalue, $\lambda_{\text {arb,sc }}$ is given by [64]

$$
\begin{aligned}
f_{\mathrm{o}, \mathrm{sc}}\left(\lambda_{\mathrm{arb}, \mathrm{sc}}\right) & =\zeta \sum_{i=1}^{m} \sum_{\substack{j=1 \\
j \neq i}}^{m} \lambda_{\mathrm{arb}, \mathrm{sc}}^{j-1} \mathcal{D}(i, j) \\
& \times\left(\theta_{n-m+i}^{n-m-1} e^{-\lambda_{\mathrm{arb}, \mathrm{sc}} / \theta_{n-m+i}}-\sum_{l=1}^{n-m} \sum_{\substack{k=1 \\
k \neq l}}^{n-m}[\boldsymbol{\Psi}]_{k, l}^{-1} \theta_{n-m+i}^{k-1} \theta_{l}^{n-m-1} e^{-\lambda_{\mathrm{arb}, \mathrm{sc}} / \theta_{l}}\right),
\end{aligned}
$$

with

$$
\zeta=\frac{\operatorname{det}(\boldsymbol{\Psi})}{m \prod_{k<l}^{n}\left(\theta_{l}-\theta_{k}\right) \prod_{l=1}^{m-1} l !}
$$

where $\boldsymbol{\Psi}$ is the $(n-m) \times(n-m)$ Vandermonde matrix defined as

$$
\boldsymbol{\Psi}=\left[\begin{array}{cccc}
1 & \theta_{1} & \ldots & \theta_{1}^{n-m-1} \\
\vdots & \vdots & \ddots & \vdots \\
1 & \theta_{n-m} & \ldots & \theta_{n-m}^{n-m-1}
\end{array}\right]
$$

Moreover, $\mathcal{D}(i, j)$ is the $(i, j)$-th co-factor of an $m \times m$ matrix whose $(l, k)$-th entry equals $(k-1) !\left(\theta_{n-m+l}^{n-m+k-1}-\sum_{p=1}^{n-m} \sum_{\substack{q=1 \\ q \neq p}}^{n-m}[\Psi]_{p, q}^{-1} \theta_{n-m+l}^{p-1} \theta_{q}^{n-m+k-1}\right)$.

Remark 2.4.2. The density in (2.33) is used to derive the ergodic spectral efficiency of semi-correlated MIMO channels with $L \geq M$. The joint eigenvalue density of two arbitrary eigenvalues is presented in the sequel. Note that the density in (2.33) is used later in the thesis in Chapter 4 for computation of the expected per-terminal SINR of a given terminal with downlink precoding.

Theorem 2.4.1. When $L \geq M$, and the $L \times M$ channel matrix $\boldsymbol{H}_{\mathrm{dl}}^{\mathrm{H}}=$ $\boldsymbol{H}_{\text {i.i.d. }}^{\mathrm{H}} \boldsymbol{R}^{\frac{1}{2}}$, i.e., the propagation channel undergoes semi-correlated Rayleigh fading, where $\theta_{1}, \ldots, \theta_{m}$ denote the $m$ distinct eigenvalues of $\boldsymbol{R} \neq \boldsymbol{I}_{m \times m}$, the joint density of any two (unordered) arbitrary eigenvalues, $\left(\lambda_{1}, \lambda_{2}\right)$ of $\boldsymbol{H}_{\mathrm{dl}}^{\mathrm{H}} \boldsymbol{H}_{\mathrm{dl}}$ is given 
by

$$
\begin{aligned}
f_{\mathrm{o}, \mathrm{sc}}\left(\lambda_{1}, \lambda_{2}\right)= & \hat{\chi} \sum_{i=0}^{m-1} \sum_{\substack{j=0 \\
j \neq i}}^{m-1}(-1)^{i+j-p(i, j)} \lambda_{1}^{i+n-m} \lambda_{2}^{j+n-m} \sum_{k=1}^{m} \sum_{\substack{l=1 \\
l \neq k}}^{m}(-1)^{k-1} e^{-\lambda_{1} / \theta_{k}} \\
& \times(-1)^{l-p(l)} e^{-\lambda_{2} / \theta_{l}} \operatorname{det}(\boldsymbol{\Xi})_{i, j ; k, l}
\end{aligned}
$$

where

$$
\hat{\chi}=\tilde{\chi}(-1)^{\left\lfloor\frac{m}{2}\right\rfloor}(m-2) !
$$

with

$$
\tilde{\chi}=\frac{1}{m ! \prod_{l=1}^{m}(n-l) ! \operatorname{det}(\boldsymbol{R})^{n} \prod_{k<l}^{m}\left(\frac{1}{\theta_{l}}-\frac{1}{\theta_{k}}\right)} .
$$

Furthermore,

$$
p(i, j)= \begin{cases}0 & \text { if } i>j \\ 1 & \text { if } i \leq j\end{cases}
$$

and

$$
p(l)=\left\{\begin{array}{cc}
0 & \text { if } k>l \\
1 & \text { if } k \leq l
\end{array}\right.
$$

Proof of Theorem 2.4.1. One can begin with the joint ordered density of $m$ eigenvalues $f_{\text {ord,sc }}\left(\lambda_{1}, \lambda_{2}, \cdots \geq \lambda_{m}\right)$ as stated in (2.26). To convert (2.26) to an unordered joint density of $m$ eigenvalues, the ordered density can be divided by $m$ ! with its range converted to $0<\lambda_{1}<\infty, \ldots, 0<\lambda_{m}<\infty$. This results in

$$
f_{\text {unord,sc }}\left(\lambda_{1}, \ldots, \lambda_{m}\right)=\tilde{\chi} \prod_{k=1}^{m} \lambda_{k}^{n-m} \prod_{k<l}^{m}\left(\lambda_{k}-\lambda_{l}\right) \operatorname{det}(\boldsymbol{\Xi}),
$$

where $\tilde{\chi}$ and $\boldsymbol{\Xi}$ are as defined in (2.38) and (2.26), respectively. Using the fact that

$$
\prod_{k<l}^{m}\left(\lambda_{k}-\lambda_{l}\right)=(-1)^{\left\lfloor\frac{m}{2}\right\rfloor} \sum_{\phi}(-1)^{\operatorname{per}(\phi)} \lambda_{1}^{\phi_{1}} \lambda_{2}^{\phi_{2}} \ldots \lambda_{m}^{\phi_{m}}
$$

where the summation is over all permutations $\phi$ of $\{1, \ldots, m\}$, substituting 
(2.41) into (2.40) allows one to write the joint unordered density as

$$
f_{\text {unord }, \mathrm{sc}}\left(\lambda_{1}, \ldots, \lambda_{m}\right)=\tilde{\chi} \prod_{k=1}^{m} \lambda_{k}^{n-m}(-1)^{\left\lfloor\frac{m}{2}\right\rfloor} \sum_{\phi}(-1)^{\operatorname{per}(\phi)} \lambda_{1}^{\phi_{1}} \lambda_{2}^{\phi_{2}} \ldots \lambda_{m}^{\phi_{m}} \operatorname{det}(\boldsymbol{\Xi})
$$

After some algebraic manipulation, the expression in (2.42) can be written as

$$
f_{\text {unord }, \mathrm{sc}}\left(\lambda_{1}, \ldots, \lambda_{m}\right)=\tilde{\chi}(-1)^{\left\lfloor\frac{m}{2}\right\rfloor} \sum_{\phi}(-1)^{\operatorname{per}(\phi)} \operatorname{det}(\boldsymbol{\Xi})
$$

where $[\boldsymbol{\Xi}]_{i, j}=\lambda_{i}^{\phi_{i}+n-m} e^{-\lambda_{i} / \theta_{j}}$. Now to integrate over $\lambda_{3}, \lambda_{4}, \ldots, \lambda_{m}$, one can integrate over the rows of $\boldsymbol{\Xi}$ specifically involving $\lambda_{3}, \lambda_{4}, \ldots, \lambda_{m}$. This property follows from Laplace's expansion for a determinant. Solving for the general case of row $k, r_{k}$, where $3 \leq k \leq m$, one can state

$$
\begin{aligned}
r_{k} & =\left[\int_{0}^{\infty} \lambda_{k}^{\phi_{k}+n-m} e^{-\lambda_{k} / \theta_{1}} d \lambda_{k}, \ldots, \int_{0}^{\infty} \lambda_{k}^{\phi_{k}+n-m} e^{-\lambda_{k} / \theta_{m}} d \lambda_{k}\right] \\
& =\left[\theta_{1}^{\phi_{k}+n-m+1}\left(\phi_{k}+n-m\right) !, \ldots, \theta_{m}^{\phi_{k}+n-m+1}\left(\phi_{k}+n-m\right) !\right] .
\end{aligned}
$$

Note that once integrated out, $\lambda_{3}, \ldots, \lambda_{m}$ disappear from rows $3, \ldots, m$, as they are only functions of $\phi_{3}, \ldots, \phi_{m}$. In contrast to this, rows 1 and 2 are still functions of $\left(\lambda_{1}, \phi_{1}\right)$ and $\left(\lambda_{2}, \phi_{2}\right)$, respectively. One can note that the number of permutations, $\phi$, such that $\phi_{1}=i$ and $\phi_{2}=j$ for any $i$ and $j$ are given by $(m-2)$ !. Upon reordering rows $3, \ldots, m$, such that $\phi_{3}, \ldots, \phi_{m}$ are ordered, one can use Laplace's expansion on the first two rows and write the joint density of two arbitrary eigenvalues, $\left(\lambda_{1}, \lambda_{2}\right)$ as

$$
\begin{aligned}
f_{\mathrm{o}, \mathrm{sc}}\left(\lambda_{1}, \lambda_{2}\right) & =\hat{\chi} \sum_{i=0}^{m-1} \sum_{\substack{j=0 \\
j \neq i}}^{m-1}(-1)^{i+j-p(i, j)} \lambda_{1}^{i+n-m} \lambda_{2}^{j+n-m} \sum_{k=1}^{m} \sum_{\substack{l=1 \\
l \neq k}}^{m}(-1)^{k-1} e^{-\lambda_{1} / \theta_{k}} \\
& \times(-1)^{l-p(l)} e^{-\lambda_{2} / \theta_{l}} \operatorname{det}(\boldsymbol{\Xi})_{i, j ; k, l}
\end{aligned}
$$

where each constant in $(2.45)$ is defined after (2.36). This yields the desired 
result and completes the proof.

Remark 2.4.3. The expression in (2.45) is left in terms of finite summations, where each summation is bounded by $m$. This makes the densities numerical evaluation efficient, given the complexity of the instantaneous channel correlation matrix. The derived result in (2.45) is extremely general and further applications to analysis involving complex correlated central Wishart matrices, such as the analysis of second order statistics of semi-correlated channels, leading to the variance of spectral efficiency for such channels. Moreover, in Chapter 4 of the thesis, this density is utilized when computing the downlink expected SINR of a given terminal with linear processing at a cellular BS.

Theorem 2.4.2. When $L<M$, and the $L \times M$ channel matrix $\boldsymbol{H}_{\mathrm{dl}}^{\mathrm{H}}=$ $\boldsymbol{H}_{\text {i.i.d. }}^{\mathrm{H}} \boldsymbol{R}^{\frac{1}{2}}$, i.e., undergoing semi-correlated Rayleigh fading, where $\theta_{1}, \ldots, \theta_{n}$ denote the $n$ distinct eigenvalues of $\boldsymbol{R} \neq \boldsymbol{I}_{n \times n}$, the joint density of any two (unordered) arbitrary eigenvalues, $\left(\lambda_{1}, \lambda_{2}\right)$, of $\boldsymbol{H}_{\mathrm{dl}}^{\mathrm{H}} \boldsymbol{H}_{\mathrm{dl}}$ is given by

$$
\begin{aligned}
f_{\mathrm{o}, \mathrm{sc}}\left(\lambda_{1}, \lambda_{2}\right)= & T(n-2) ! \sum_{i=0}^{m-1} \sum_{\substack{j=0 \\
j \neq i}}^{m-1}(-1)^{i+1-p(i, l)} \sum_{o=1}^{m}(-1)^{o-1} \theta_{o}^{n-m-1} \lambda_{1}^{i} e^{-\lambda_{1} / \theta_{0}} \\
& \times \sum_{\substack{p=1 \\
p \neq o}}^{m}(-1)^{p-p(o)} \theta_{p}^{n-m-1} \lambda_{2}^{j} e^{-\lambda_{2} / \theta_{p}} \boldsymbol{\Theta}
\end{aligned}
$$

where $T$ is defined in (2.30), $p(i, l)$ and $p(o)$ are equivalently defined in (2.39), while $\boldsymbol{\Theta}=\operatorname{det}\left(\left[\boldsymbol{\Delta}_{n}\right]_{o ; p}\left[\boldsymbol{\Delta}_{n, m}\right]_{o, p ; i, j}\right)$.

Proof of Theorem 2.4.2. One can begin with the joint density of $m$ distinct eigenvalues given by [65]

$$
f_{\text {unord,sc }}\left(\lambda_{1}, \ldots, \lambda_{m}\right)=T \sum_{\phi}(-1)^{\operatorname{per}(\phi)} \prod_{i=1}^{m} \lambda_{i}^{\phi_{i}} \operatorname{det}(\boldsymbol{\Xi}),
$$

Integrating over $\lambda_{3}, \ldots, \lambda_{m}$ in (2.47) yields,

$$
f_{\mathrm{o}, \mathrm{sc}}\left(\lambda_{1}, \lambda_{2}\right)=\frac{(n-2) !}{\prod_{j=1}^{m} j !} \sum_{i=0}^{m-1} \sum_{\substack{l=0 \\ l \neq i}}^{m-1}(-1)^{i+l-p(i, l)} \operatorname{det}\left(\boldsymbol{\Delta}[\boldsymbol{\Xi}]_{i ; l}\right),
$$


where $[\boldsymbol{\Xi}]_{i ; l}$ is equivalent to $\boldsymbol{\Xi}$ with columns $i$ and $l$ ordered corresponding to $\lambda_{1}$ and $\lambda_{2}$. Upon reordering, performing a Laplace expansion on the $i$-th column with $\lambda_{1}$, one can obtain

$$
\begin{aligned}
f_{\mathrm{o}, \mathrm{sc}}\left(\lambda_{1}, \lambda_{2}\right)= & \frac{(n-2) !}{\prod_{j=1}^{m} j !} \sum_{i=0}^{m-1} \sum_{\substack{l=0 \\
l \neq i}}^{m-1}(-1)^{i+l-p(i, l)}(-1)^{n-m} \sum_{o=1}^{m}(-1)^{o-1} \\
& \times \theta_{o}^{n-m-1} \lambda_{1}^{i} e^{-\lambda_{1} / \theta_{o}} \operatorname{det}\left(\boldsymbol{\Delta}_{o}[\boldsymbol{\Xi}]_{i, l ; o}\right) .
\end{aligned}
$$

Performing a second Laplace expansion with the determinant in (2.48) with $\lambda_{2}$ and the $j$-th column yields the expression in (2.46).

Remark 2.4.4. The same comments in Remark 2.4.3. applies to the result derived in (2.47). Following the results for the uncorrelated and correlated complex central Wishart matrices, the arbitrary and joint pair of arbitrary eigenvalue densities of uncorrelated non-central Wishart matrices are presented in the following subsection.

\subsubsection{Uncorrelated Non-Central Wishart Matrices}

Definition 2.4.7. When $\boldsymbol{H}_{\mathrm{dl}}^{\mathrm{H}}$ undergoes uncorrelated Ricean fading, $\boldsymbol{H}_{\mathrm{dl}}^{\mathrm{H}} \boldsymbol{H}_{\mathrm{dl}}$ follows an uncorrelated non-central Wishart structure with $m$ degrees of freedom and a covariance matrix of $\boldsymbol{I}_{m}$. For the case $L<M$, the joint density of $m$ eigenvalues of $\boldsymbol{H}_{\mathrm{dl}}^{\mathrm{H}} \boldsymbol{H}_{\mathrm{dl}}$ in $\lambda_{1} \geq \lambda_{2} \geq, \ldots, \geq \lambda_{m}$ is given by [54]

$$
\begin{aligned}
f_{\text {ord,nonc }}\left(\lambda_{1}, \ldots, \lambda_{m}\right)= & \frac{e^{-\operatorname{tr}\left[K \overline{\boldsymbol{H}}_{\mathrm{d} \mathbf{H}}^{\mathrm{H}}\right]}}{\prod_{l=1}^{m}(n-l) !(m-l) !} \prod_{k=1}^{m} \lambda_{k}^{n-m}{ }_{0} F_{1}\left(n, K, \overline{\boldsymbol{H}}^{\mathrm{H}} \overline{\boldsymbol{H}}, \boldsymbol{\Lambda}\right) \\
& \times e^{-\operatorname{tr}[\boldsymbol{\Lambda}]} \prod_{k<l}^{m}\left(\lambda_{k}-\lambda_{l}\right)^{2},
\end{aligned}
$$

where $K$ is the Rice factor and $\boldsymbol{\Lambda}=\operatorname{diag}\left(\lambda_{1}, \ldots, \lambda_{m}\right)$, a diagonal matrix of $m$ eigenvalues. Furthermore, one can express ${ }_{0} F_{1}\left(n, K, \overline{\boldsymbol{H}}^{\mathrm{H}} \overline{\boldsymbol{H}}, \boldsymbol{\Lambda}\right)$, the hypergeo- 
metric function containing matrix arguments as

$$
\begin{aligned}
{ }_{0} F_{1}\left(n, K, \overline{\boldsymbol{H}}^{\mathrm{H}} \overline{\boldsymbol{H}}, \boldsymbol{\Lambda}\right) & = \\
& \frac{\prod_{l=1}^{m-1}(m-l)^{l}(n-l)^{l}}{\prod_{k<l}^{m}\left(\phi_{k}-\phi_{l}\right)}\left[\frac{\operatorname{det}\left(\left\{{ }_{0} F_{1}\left(n-m+1, \phi_{i}, \lambda_{j}\right)\right\}\right)}{\prod_{k<l}^{m}\left(\lambda_{k}-\lambda_{l}\right)}\right],
\end{aligned}
$$

where $\operatorname{det}\left(\left\{a_{i, j}\right\}\right)$ is the determinant of a matrix whose $(i, j)$-th entry is $a_{i, j}$, while ${ }_{0} F_{1}(\cdot, \cdot)$ is the scalar hypergeometric function. Moreover, $\phi_{1}, \ldots, \phi_{m}$ are the $m$ eigenvalues of $\sqrt{K} \overline{\boldsymbol{H}}$.

Definition 2.4.8. Under the same conditions as 2.4.7, the arbitrary eigenvalue density, $\lambda_{\text {nonc,arb }}$, of the uncorrelated non-central Wishart matrix, $\boldsymbol{H}_{\mathrm{dl}}^{\mathrm{H}} \boldsymbol{H}_{\mathrm{dl}}$ can be written as [66],

$$
\begin{gathered}
f_{\mathrm{o}}\left(\lambda_{\text {arb,nonc }}\right)=\frac{e^{-\sum_{i} \phi_{i}}}{m((n-m) !)^{m}} \frac{e^{-\lambda_{\text {arb,conc }}(K+1)}}{\lambda_{\text {arb,conc }}} \sum_{j=1}^{m}\left((K+1) \lambda_{\text {arb,conc }}\right)^{n-m+j} \\
\sum_{i=1}^{m} \frac{{ }_{0} F_{1}\left(n-m+1,(K+1) \phi_{i} \lambda_{\text {arb,conc }}\right) \mathcal{D}(i, j)}{\prod_{k<l}^{m}\left(\phi_{l}-\phi_{k}\right)}
\end{gathered}
$$

where $\mathcal{D}(i, j)$ is the $(i, j)$-th co-factor of the $m \times m$ matrix $\boldsymbol{A}$ whose $(l, k)$-th entry is given by

$$
(\boldsymbol{A})_{l, k}=(n-m+k-1) !{ }_{1} F_{1}\left(n-m+k, n-m+1, \phi_{l}\right),
$$

with and ${ }_{1} F_{1}(\cdot, \cdot, \cdot)$ is the Kummer confluent hypergeometric function [66]. Next, the novel result on the joint density of two arbitrary eigenvalues is presented and derived.

Theorem 2.4.2. When $L \leq M$, and the $L \times M$ channel matrix follows the uncorrelated Ricean fading distribution following (2.17). Then, the joint density of any two (arbitrary) eigenvalues of $\boldsymbol{H}_{\mathrm{dl}}^{\mathrm{H}} \boldsymbol{H}_{\mathrm{dl}}$ is given by

$$
f_{\text {o,nonc }}\left(\lambda_{1}, \lambda_{2}\right)=C \sum_{i=0}^{m-1} \sum_{\substack{j=0 \\ j \neq i}}^{m-1} \sum_{r=1}^{m} \sum_{\substack{s=1 \\ s \neq r}}^{m}(-1)^{u} \widetilde{\boldsymbol{\Xi}}(r, s ; i, j) g_{r, i}\left(\lambda_{1}\right) g_{s, j}\left(\lambda_{2}\right),
$$


where $u=i+j+r+s-p(i, j)-t(r, s)$ with

$$
p(i, j)=\left\{\begin{array}{l}
0 ; j \leq i \\
1 ; j>i
\end{array}\right.
$$

and

$$
t(r, s)=\left\{\begin{array}{l}
0 ; s \leq r \\
1 ; s>r
\end{array}\right.
$$

Furthermore,

$$
C=\frac{e^{-\sum_{i} \phi_{i}}}{m((n-m) !)^{m} \prod_{k<q}^{m}\left(\phi_{q}-\phi_{k}\right)}((n-m) !)^{m}(-1)^{\left\lfloor\frac{m}{2}\right\rfloor}(n-2) !,
$$

and

$$
\widetilde{\Xi}(r, s ; i, j)=\left(\phi_{r} \phi_{s}\right)^{-v / 2} \boldsymbol{\Xi}(r, s ; i, j) \text {. }
$$

In addition to this,

$$
g_{a, b}(\lambda)=\lambda^{v / 2+b} e^{-\lambda} \mathrm{I}_{v}\left(2 \sqrt{\phi_{a} \lambda}\right)
$$

where $v=n-m$. In (2.57), $\boldsymbol{\Xi}(r, s ; i, j)$ is a determinant with rows $r, s$ and columns $i, j$ removed, where the $d$-th entry of the $f$-th column is given by $\frac{\Gamma\left(\varsigma+\varrho_{f}\right)}{\Gamma(\varsigma)}{ }_{1} F_{1}\left(\varsigma+\varrho_{f}, \varsigma, \phi_{d}\right)$. Here, $\varrho_{f}=f-1, \Gamma(\cdot)$ is the scalar gamma function, and $\mathrm{I}_{v}(\cdot)$ is the modified Bessel function of the first kind $[18,63]$.

Proof of Theorem 2.4.2. Invoking Appendix A.2 of [66], the joint unordered density of the $m$ eigenvalues, $\lambda_{1}, \ldots, \lambda_{m}$ of the complex uncorrelated non-central Wishart matrix is given by

$$
\begin{aligned}
& f_{\text {unord,nonc }}\left(\lambda_{1}, \ldots, \lambda_{m}\right)=\frac{e^{-\sum_{i} \phi_{i}}}{m((n-m) !)^{m} \prod_{k<q}^{m}\left(\phi_{q}-\phi_{k}\right)} \prod_{k<q}^{m}\left(\lambda_{k}-\lambda_{q}\right) \\
& \prod_{k=1}^{m} \lambda_{k}^{n-m} e^{-\sum_{i=1}^{m} \lambda_{i}} \boldsymbol{\Xi}_{1} \text {, }
\end{aligned}
$$

where $\boldsymbol{\Xi}_{1}$ is the determinant of ${ }_{0} F_{1}\left(n-m+1, \phi_{i} \lambda_{j}\right)$. Recognizing that ${ }_{0} F_{1}(a, b)=$ $(a-1) ! b^{-(a-1) / 2} \mathrm{I}_{a-1}(2 \sqrt{b})$ and decomposing the first product in (2.59) into a 
summation over permutations of $\varrho$ from $0,1, \ldots, m-1$, one can write $(2.59)$ as

$$
\begin{aligned}
f_{\text {unord,nonc }}\left(\lambda_{1}, \ldots, \lambda_{m}\right)= & \frac{e^{-\sum_{i} \phi_{i}}}{m((n-m) !)^{m} \prod_{k<q}^{m}\left(\phi_{q}-\phi_{k}\right)} \\
& ((n-m) !)^{m}(-1)^{\left\lfloor\frac{m}{2}\right\rfloor} \sum_{\varrho}(-1)^{\operatorname{per}(\varrho)} \Xi_{2},
\end{aligned}
$$

where $\boldsymbol{\Xi}_{2}$ is the determinant of $\phi_{i}^{-\frac{n-m}{2}} \lambda_{j}^{\frac{n-m}{2}+\varrho_{j}} e^{-\lambda_{j}} \mathrm{I}_{n-m}\left(2 \sqrt{\phi_{i} \lambda_{j}}\right)$. To find $f_{\text {o,nonc }}\left(\lambda_{1}, \lambda_{2}\right)$, one can integrate over $\lambda_{3}, \ldots, \lambda_{m}$, i.e., over columns $3, \ldots, m$ of $\boldsymbol{\Xi}_{2}$. The resulting entries of $\boldsymbol{\Xi}_{2}$ can now be written as

$$
\frac{\Gamma\left(n-m+1+\varrho_{j}\right)}{\Gamma(n-m+1)}{ }_{1} F_{1}\left(n-m+1+\varrho_{j}, n-m+1, \phi_{i}\right) .
$$

Reordering the columns of $\boldsymbol{\Xi}_{2}$, such that $\varrho$ is ordered from $0,1, \ldots, m-1$ and performing the Laplace expansion on columns $i, j$, containing $\lambda_{1}$ and $\lambda_{2}$, yields

$$
\begin{aligned}
f_{\mathrm{o}, \text { nonc }}\left(\lambda_{1}, \lambda_{2}\right)= & \frac{e^{-\sum_{i} \phi_{i}}}{m((n-m) !)^{m} \prod_{k<q}^{m}\left(\phi_{q}-\phi_{k}\right)}((n-m) !)^{m}(-1)^{\left\lfloor\frac{m}{2}\right\rfloor}(n-2) ! \\
& \sum_{i=0}^{m-1} \sum_{\substack{j=0 \\
j \neq i}}^{m-1} \sum_{r=1}^{m} \sum_{\substack{s=1 \\
s \neq r}}^{m}(-1)^{u} \boldsymbol{\Xi}(r, s ; i, j) \phi_{r}^{-v / 2} \lambda_{1}^{v / 2+i} e^{-\lambda_{1}} \mathrm{I}_{v}\left(2 \sqrt{\phi_{r} \lambda_{1}}\right) \\
& \phi_{s}^{-v / 2} \lambda_{2}^{v / 2+j} e^{-\lambda_{2}} \mathrm{I}_{v}\left(2 \sqrt{\phi_{s} \lambda_{2}}\right) .
\end{aligned}
$$

Further simplifying and denoting $u=i+j+r+s-p(i, j)-t(r, s), v=n-m$, $\widetilde{\boldsymbol{\Xi}}(r, s ; i, j)=\left(\phi_{r} \phi_{s}\right)^{-v / 2} \boldsymbol{\Xi}(r, s ; i, j)$ and $g_{a, b}(\lambda)=\lambda^{v / 2+b} e^{-\lambda} \mathrm{I}_{v}\left(2 \sqrt{\phi_{a} \lambda}\right)$ yields the expression in (2.54). This concludes the proof.

Remark 2.4.5. As it will be seen in Chapter 5 of the thesis, Theorem 2.4 .2 is used to compute the expected per-terminal SINR with linear processing on the downlink of a multiuser MIMO system, where each link undergoes uncorrelated Ricean fading. Furthermore, the result derived in (2.54) has general applicability for analysis involving the complex uncorrelated non-central Wishart matrix. Prior works (see $[67,68]$ and references therein) often approximate the noncentrality of the Wishart matrix by its central counterpart, via an adjustment of its covariance matrix. In contrast to this, the derived result omits the need 
for this conversion and allows one to analyze the non-central Wishart structure in its exact form for further analysis.

This concludes the first background chapter in the thesis covering the statistical channel models and random matrix theory results used throughout the thesis. The following chapter (Chapter 3) presents an introduction to multiuser MIMO systems and the associated linear transceiver structures which are analyzed in the contribution chapters (Chapters 4-8) of the thesis. 


\section{Chapter 3}

\section{Multiuser MIMO Systems}

Summary. The main focus of this chapter is on multiuser multiple-input multiple-output (MU-MIMO) systems, where an antenna array simultaneously serves multiple user terminals in the same time and frequency resource. With such an ability, MU-MIMO systems have become part of many standardized technologies, such as Long-Term Evolution-Advanced (LTE-A), and are envisioned to be the workhorse of next generation cellular systems. An overview of the downlink and uplink MU-MIMO systems along with their assumptions is presented, followed by a discussion on the heterogeneity introduced by MUMIMO systems with scenarios that are of current and future interest. As serving multiple terminals in the same time-frequency resource gives rise to multiuser interference, the chapter details uplink and downlink linear signal processing techniques to mitigate or suppress the effects of multiuser interference. An introduction to the important performance metrics of the expected signal-tointerference-plus-noise-ratio and ergodic sum spectral efficiency is presented, followed by a discussion on the artifacts of large MU-MIMO systems, where the number of transmit antennas are increased without bound, serving a finite number of user terminals. To minimize the detrimental impact of antenna correlation and other electromagnetic coupling between adjacent elements at the transmitter, wide antenna separations are required. However, in order to maintain a fixed form factor, large antenna arrays should be designed in confined physical volumes. To this end, large MU-MIMO systems operating in fixed 
physical spaces are introduced. Overall, this chapter facilitates the analysis and discussion presented in later chapters of the thesis, by introducing the key concepts related to MU-MIMO systems.

\subsection{System Operation and Assumptions}

Providing an ever increasing amount of spectral efficiency in a designated area with limited resources is one of the most fundamental problems in cellular communication systems $[9,69,70]$. The majority of the proposed solutions fall into three categories: (1) Exploitation of spectrum that is currently unused or underutilized; (2) Deployment of more cellular base stations (BSs) serving a commensurately smaller area; (3) Use of cellular BSs with multiple antenna elements. ${ }^{1}$ For future cellular systems, the first two approaches will be epitomized by millmeter-wave (mmWave) carrier frequencies operating in smaller cells. The third approach is known as multiple-input multiple-output (MIMO) [5-7], or multiuser MIMO (MU-MIMO) [10,15], of which large (a.k.a. massive) MIMO is rapidly emerging as a popular form $[9,11,57,71]$.

Fig. 3.1 demonstrates the features of a MU-MIMO system. For simplicity, the system is shown for an isolated BS site and several single-antenna terminals. ${ }^{2}$ Here, downlink transmission is considered, where an array of $M$ antennas serve a multiplicity of autonomous, non-cooperative user terminals. The main difference between a point-to-point MIMO system described in Chapter 2, and a MU-MIMO system is the decomposition of a point-to-point MIMO link into $L$ multiple-input single-output links. Here, $L$ is the total number of terminals being served. Due to the physical constraints on its size, the terminals are assumed to have a single-antenna each. For the purpose of the chapter and the thesis, ideal antenna elements configured in a uniform linear array (ULA) are assumed at the cellular BS (serving array of antennas). Perfect hardware is also

\footnotetext{
${ }^{1}$ In practice, the user terminal can also be equipped with multiple antennas, however, for this discussion, it is assumed that each terminal will only have a single-antenna and will be allocated one stream of data.

${ }^{2}$ In reality, multiple cells are are always present in a network of cells. However, for ease of exposition here, the author focuses on the case where only one BS is considered.
} 


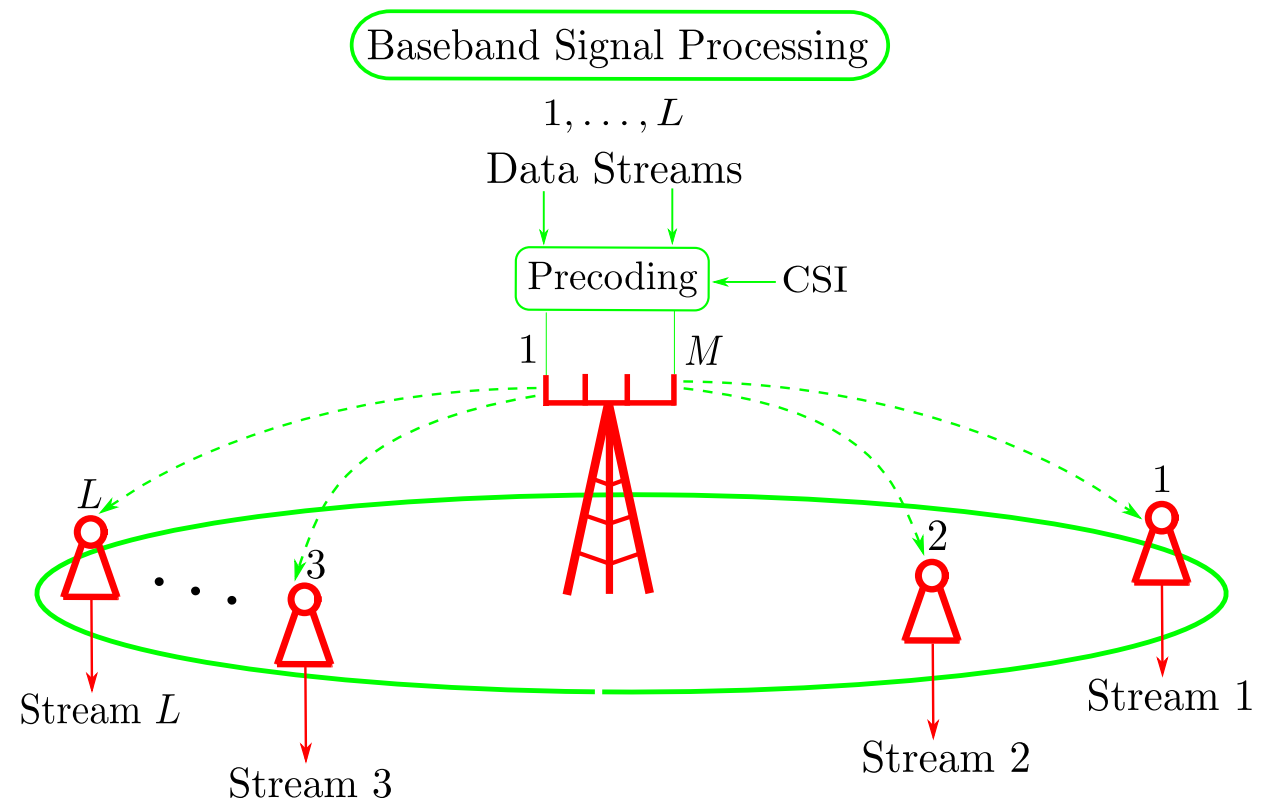

Figure 3.1: A classical, fully digital downlink MU-MIMO system. The antenna array selectively transmits a multiplicity of data streams, all occupying the same resource in time and frequency.

assumed at each user terminal. The above assumptions are a result of the thesis primarily focusing on the performance analysis of MU-MIMO systems with a wide range of practically feasible transceiver structures, operating in heterogeneous channel conditions (described further in Section 3.4). As such, the focus is not on the system level imperfections or on a specific transceiver type, where considering the above imperfections are imperative. Here, the author is interested in tackling the more general and fundamental problem of mathematically characterizing the spectral efficiency performance of MU-MIMO systems over a wide range of scenarios and fading channels, where it is sensible to make such idealistic assumptions to make analytical progress.

The downlink operation of the system entails transmitting $L$ data stream to $L$ terminals. Due to this being a broadcast system, serving multiple terminals simultaneously leads to multiuser interference (also known as inter-user interference), as the desired data stream to a given terminal will be unwanted at all other terminals. Hence, the main objective of a MU-MIMO system is to ensure that each terminal receives the desired data stream, with minimal levels of multiuser interference. It is worth noting that contemporaneous cellular sys- 


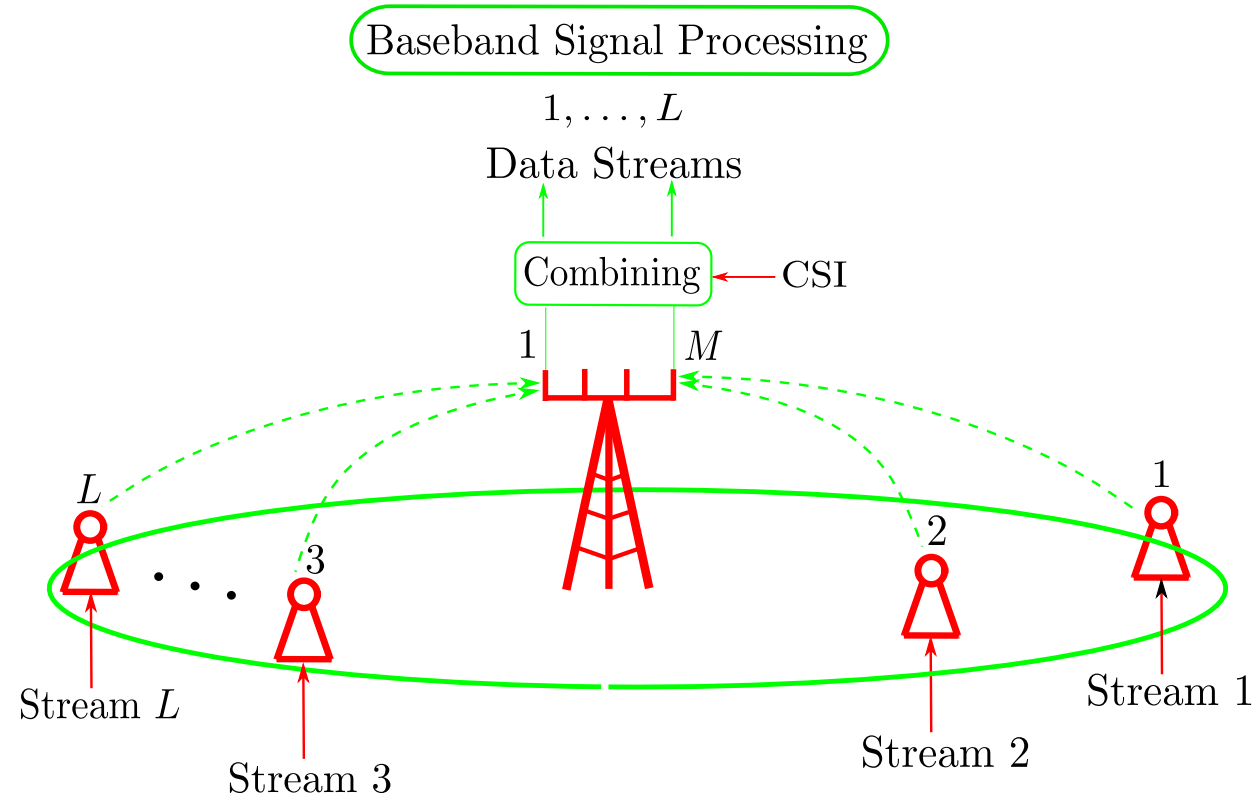

Figure 3.2: A classical, fully digital uplink MU-MIMO system. The terminals transmit data which occupy the same time-frequency resources and the transmitted signals received by the elements of the serving array are processed to recover the individual data streams.

tems typically accomplish this multiplexing by some combination of sending the various data streams at different times (known as time-division multiplexing) and with different frequencies (known as frequency-division multiplexing), both of which lead to a degradation of spectral efficiency and inefficient use of radio resources. In stark contrast to this, MU-MIMO systems use space-division multiplexing (also known as space-division multiple access), such that the different data streams occupy the same time and frequency resource. As mentioned in Chapter 2, classical orthogonal frequency-division multiplexing (OFDM) waveforms across time and frequency are assumed, with narrowband signaling within each OFDM subcarrier. ${ }^{3}$

A key element to performing spatial multiplexing is an array of independently controlled service antennas at the BS. Under line-of-sight (LoS) propagation, the data streams are carried on focused beams of data. In a heavily scattered propagation conditions (statistically often modeled via Rayleigh fading), the data

\footnotetext{
${ }^{3}$ Note that the OFDM subcarrier index is omitted for simplicity in all presented downlink and uplink performance metrics, as well as for all multiuser signal processing techniques, in Sections 3.3 and 3.4, respectively.
} 
streams can arrive from many directions simultaneously. Assuming that the necessary baseband signal processing is performed at the BS, the data streams can be combined in order to boost the desired signal and reduce interference. Moreover, a critical prerequisite to execute and achieve MU-MIMO spatial multiplexing is that the service array needs to have knowledge of the gains of each downlink channel, i.e., from each of its elements to all terminals. This is known as channel state information (CSI), and is utilized in baseband signal processing at the service array to perform downlink precoding to each terminal. ${ }^{4}$

Downlink precoding is a way to mitigate or reduce the effects of multiuser interference while serving multiple terminals in the same radio resource. As the prime focus of the thesis is on the performance analysis of MU-MIMO systems under extremely general channel conditions, perfect knowledge of the CSI is assumed both at the terminals and at the service array. The data streams are simultaneously mapped onto the signals that drive each service antenna. ${ }^{5}$ It is also worth noting that all other system blocks demonstrated in Chapter 2 for point-to-point MIMO are still in place but omitted from Fig. 3.1 to avoid cluttering the diagram. On the other hand, uplink operation of the MU-MIMO system, as shown in Fig. 3.2, is essentially the reverse of downlink operation. Here the terminals transmit their own data streams at the same time and over the same frequency resources. The BS array receives the sum of the data streams as modified by their respective propagation channels, and the combining operation, again utilizing the complete CSI, untangles the received signals to produce the individual data streams.

Having mentioned the basic operations, assumptions and key aspects of MUMIMO systems considered in the thesis, the following section outlines the physical implications enforced on MU-MIMO systems by the propagation channels they operate in.

\footnotetext{
${ }^{4}$ The author would like to note that acquisition of CSI is an important research topic in its right. Research is actively being performed with both time-division and frequency-division duplex protocols. It remains an open question as to which duplexing mode should be used to acquire CSI at the BS. The ultimate answer to this question may be scenario dependent and relies on many other factors which are outside the scope of the thesis.

${ }^{5}$ By increasing the sheer number of elements at the BS array, as in large MU-MIMO, the beams can be focused more selectively to the desired terminal.
} 


\subsection{Heterogeneity in MU-MIMO Systems}

With spatial separation of user terminals within the vicinity of the BS coverage area, MU-MIMO systems experience a diverse range of propagation conditions. Depending on the geographical terrain around a given terminal, each terminal experiences a unique link gain composing the effects of geometric attenuation and shadow-fading. ${ }^{6}$ With a unique set of link gains for each terminal, the analysis of MU-MIMO systems is mathematically more challenging than for pointto-point MIMO systems, where the receive antennas are co-located. In addition to this, small-scale fading co-efficients also randomly vary due to the location differences of multiple terminals. The standard statistical models introduced in Chapter 2 are directly applicable in this context.

With the rise of small-cellular systems illuminating smaller coverage areas, the average link distance between a terminal and the BS is reduced in comparison to conventional macro-cellular systems. For a particular terminal, this is likely to result in higher probability of LoS, where the propagation channel is insufficiently dominated by rich scattering. Furthermore, the local terrain variations within the proximity of a particular terminal result in a change in the amount of LoS present on a particular link. In turn, this means that both the LoS and the non LoS (NLoS) powers are a function of the terrain variation across multiple terminals. Statistically, the above scenario can not be modeled with the classical assumption of uncorrelated, independent and identically distributed (i.i.d.) Rayleigh fading. In contrast to this, a Ricean fading model with dominant LoS directions in addition to the contribution from the scattered components is better suited to capture such environments. In particular, such scenarios should employ the Ricean fading channel model with a terrain dependent $K$-factor to capture the heterogeneity of MU-MIMO channels.

Moreover, it is envisioned that that small-cellular systems may be complemented by the use of mmWave carrier frequencies for future terrestrial systems. Here, to overcome the large propagation losses at such high carrier frequen-

\footnotetext{
${ }^{6}$ The statistical model for link gain is presented in Section 2.1.2 in Chapter 2.
} 
cies, future cellular BSs may be equipped with large antenna arrays, needed to provide the necessary array gain for quality-of-service. In order to achieve the conventional inter-element spacing of more than half wavelength which is known to induce less antenna correlation and electromagnetic coupling, large antenna arrays require a much larger physical space in comparison to conventional arrays. Nevertheless, the physical size of the array should also be constrained before it becomes impractical to deploy such arrays in certain scenarios, such as dense urban, where ideally the BS array should occupy a relatively smaller spacing. Accommodating a large number of serving antennas in confined physical volumes leads to an increased level of spatial correlation and electromagnetic coupling, which have a detrimental impact on the performance of a given terminal and the system. In addition to the small inter-element distances, spatial correlation is also a function of the angular spread at a given terminal. A moderate change in the terminal's location implies a change in the local terrain around the terminal. This leads to a further change in the correlation pattern, due to the change in the distribution of the incoming wavefronts, as the BS array may illuminate a different set of scatterers in the propagation channel. Considering this, two terminals, far apart, can experience vastly different spatial correlation patterns. Due to this reason, unequal levels of correlation are to be expected in future cellular systems. ${ }^{7}$

The fusion of all three technologies, namely small-cellular, mmWave, and large antenna arrays implies heterogeneous propagation channels, which contain unequal levels of LoS, unequal levels of spatial correlation, unequal levels of link gains, and unequal instantaneous small-scale fading. As such, performance analysis of MU-MIMO systems considering these variations is timely, and of great significance. Such a general structure of the propagation channel makes performance analysis of MU-MIMO systems with transmit precoding and receive combining extremely challenging. Despite knowledge of the above physical artifacts induced by the propagation channel, researchers have found it difficult to make analytical progress to predict the spectral efficiency performance of

\footnotetext{
${ }^{7}$ Naturally, the terminals have to lie within the radius of the serving array's coverage area.
} 
MU-MIMO systems in such situations. As a result, analyzing the performance of current and future MU-MIMO systems with heterogeneous channels is the central theme of the thesis, as demonstrated in Chapters 4-8.

In what follows, a comprehensive overview of the uplink and downlink MUMIMO signal model is given.

\subsection{Signal Models}

Considering the multiuser system described in Section 3.1, let $\boldsymbol{G}_{\mathrm{ul}}$ be the $M \times L$ composite uplink propagation channel matrix between the $M$ BS antennas at the ULA and $L$ terminals in the system. A circular coverage area of radius $R_{\mathrm{c}}$ meters is assumed around the BS, which is located at the origin of the circle. Note that $\boldsymbol{g}_{\mathrm{ul}, l}$ denotes the $M \times 1$ uplink channel vector from the $l$-th terminal to the BS, and can be written as

$$
\boldsymbol{g}_{\mathrm{ul}, l}=\boldsymbol{h}_{\mathrm{ul}, l} \beta_{l}^{\frac{1}{2}}
$$

where $\beta_{l}$ is the link gain for terminal $l$ composed of the large-scale fading effects of geometric attenuation and shadow-fading. In particular,

$$
\beta_{l}=v\left(\frac{d_{0}}{d_{l}}\right)^{\alpha} \zeta_{l}
$$

where $v$ is the unit-less constant for geometric attenuation at a given reference distance, $d_{0}, d_{l}$ is the link distance from the BS to the $l$-th terminal. Moreover, $\alpha$ is the geometric attenuation exponent and $10 \log _{10}\left(\zeta_{l}\right) \sim \mathcal{N}\left(0, \sigma_{\text {sf }}^{2}\right)$ with $\sigma_{\text {sf }}$ denoting the shadow-fading standard deviation.

The nature of $\boldsymbol{h}_{\mathrm{ul}, l}$ depends on the channel conditions. For ease of exposition, uncorrelated i.i.d. Rayleigh fading, i.e., $\boldsymbol{h}_{\mathrm{ul}, l} \sim \mathcal{C N}\left(0, \boldsymbol{I}_{M}\right)$ is considered in this chapter for the purpose of introducing uplink and downlink transmission, the relevant multiuser signal processing techniques, as well as large MU-MIMO systems. It is worth noting that even though i.i.d. Rayleigh fading channels are used for the remainder of the chapter, the mathematical system description, 
and multiuser processing approaches remain exactly the same for any type of fading channel model.

In what follows, uplink and downlink transmission modes of the MU-MIMO system are presented.

\subsubsection{Uplink Transmission}

As mentioned in Section 3.1, on the uplink, $L$ terminals transmit data streams simultaneously in the same time-frequency resource up to the BS. Note that $s_{\mathrm{ul}, l}$ is denoted as the normalized transmitted data symbol from the $l$-th terminal, such that $\mathbb{E}\left[\left|s_{\mathrm{ul}, l}\right|^{2}\right]=1$. Then, the $M \times 1$ received signal at the $\mathrm{BS}$ array is a combination all transmitted signals from all $L$ terminals, and is given by

$$
\begin{aligned}
\boldsymbol{y}_{\mathrm{ul}} & =\rho_{\mathrm{ul}}^{\frac{1}{2}} \sum_{l=1}^{L} \boldsymbol{g}_{\mathrm{ul}, l} s_{\mathrm{ul}, l}+\boldsymbol{n}_{\mathrm{ul}} \\
& =\rho_{\mathrm{ul}}^{\frac{1}{2}} \boldsymbol{G}_{\mathrm{ul}} \boldsymbol{s}_{\mathrm{ul}}+\boldsymbol{n}_{\mathrm{ul}},
\end{aligned}
$$

where $\rho_{\mathrm{ul}}^{\frac{1}{2}}$ is the average uplink transmit power of a given terminal, $\boldsymbol{G}_{\mathrm{ul}}=$ $\left[\boldsymbol{g}_{\mathrm{ul}, 1}, \ldots, \boldsymbol{g}_{\mathrm{ul}, L}\right], \boldsymbol{s}_{\mathrm{ul}}=\left[s_{\mathrm{ul}, 1}, s_{\mathrm{ul}, 2}, \ldots, s_{\mathrm{ul}, L}\right]^{\mathrm{T}}$ is the $L \times 1$ vector of transmitted data symbols from all terminals. Moreover, $\boldsymbol{n}_{\mathrm{ul}}=\left[n_{\mathrm{ul}, 1}, n_{\mathrm{ul}, 2}, \ldots, n_{\mathrm{ul}, M}\right]^{\mathrm{T}}$ is the $M \times 1$ vector of additive white Gaussian noise terms at the BS array elements, where $n_{\mathrm{ul}, m} \sim \mathcal{C N}\left(0, \sigma_{\mathrm{ul}, m}^{2}\right)$. Note that $\sigma_{\mathrm{ul}, 1}^{2}=, \ldots,=\sigma_{\mathrm{ul}, M}^{2}=\sigma_{\mathrm{ul}}^{2}$ is assumed. Thus, the link SNR for an arbitrary link from a terminal to the BS array is denoted by the ratio of the average uplink transmit power to the noise power at the BS array, i.e., $\rho_{\mathrm{ul}} / \sigma_{\mathrm{ul}}^{2}{ }^{8}$

For such composite uplink channel matrices, the Shannon sum spectral efficiency is given by [36]

$$
\mathrm{R}_{\mathrm{ul}, \mathrm{sum}}=\log _{2}\left(\operatorname{det}\left(\boldsymbol{I}_{L}+\frac{\rho_{\mathrm{ul}} L}{\sigma_{\mathrm{ul}}^{2}} \boldsymbol{G}_{\mathrm{ul}} \boldsymbol{G}_{\mathrm{ul}}^{\mathrm{H}}\right)\right)
$$

\footnotetext{
${ }^{8}$ It is also worth noting that even though the additive noise at the BS array is drawn from the same distribution as the small-scale fading, it is independent of the small-scale fading channel entries.
} 
For completeness, it is worth mentioning that the aforementioned sum spectral efficiency can be achieved by using the successive interference cancellation (SIC) technique [72]. ${ }^{9}$ With SIC, after one terminal is detected at the BS array, its signal is subtracted from the received signal before the next terminal is detected.

\subsubsection{Downlink Transmission}

In contrast to the uplink, when the BS array transmits signals on the downlink to all $L$ terminals, the information is carried in the $M \times 1$ vector of the transmitted symbols $\boldsymbol{s}_{\mathrm{dl}}$, where $\mathbb{E}\left[\|\left.\boldsymbol{s}_{\mathrm{dl}}\right|^{2}\right]=1$. Hence, the downlink received signal at the $l$-th terminal is given by

$$
y_{\mathrm{dl}, l}=\rho_{\mathrm{dl}}^{\frac{1}{2}} \boldsymbol{g}_{\mathrm{dl}, l}^{\mathrm{H}} \boldsymbol{s}_{\mathrm{dl}}+n_{\mathrm{dl}, l},
$$

where $\rho_{\mathrm{dl}}^{\frac{1}{2}}$ is the average downlink transmit power at the BS, $\boldsymbol{g}_{\mathrm{dl}, l}^{\mathrm{H}}$ is the $1 \times M$ downlink channel vector to the $l$-th terminal from the BS array, and $n_{\mathrm{dl}, l}$ is the additive white Gaussian noise at the input of the $l$-th terminal, such that $n_{\mathrm{dl}, l} \sim$ $\mathcal{C N}\left(0, \sigma_{\mathrm{dl}, 1}^{2}\right)$. As for the uplink case, it is assumed that $\sigma_{\mathrm{dl}, 1}^{2},=\ldots=, \sigma_{\mathrm{dl}, L}^{2}=\sigma_{\mathrm{dl}}^{2}$, and hence the link SNR for an arbitrary link is given by the ratio of $\rho_{\mathrm{dl}}$ to $\sigma_{\mathrm{dl}}^{2}$, i.e., $\rho_{\mathrm{dl}} / \sigma_{\mathrm{dl}}^{2}$. Collectively, the $L \times 1$ received signal vector at all $L$ terminals can be written as

$$
\boldsymbol{y}_{\mathrm{dl}}=\rho_{\mathrm{dl}}^{\frac{1}{2}} \boldsymbol{G}_{\mathrm{dl}}^{\mathrm{H}} \boldsymbol{s}_{\mathrm{dl}}+\boldsymbol{n}_{\mathrm{dl}} .
$$

The composite downlink channel is also known as the broadcast channel, whose sum spectral efficiency (Shannon sum spectral efficiency) is known to be [73]

$$
\mathrm{R}_{\mathrm{dl}, \mathrm{sum}}=\max _{\substack{\left\{q_{l}\right\} \\ q_{l} \geq 0, \sum_{l=1}^{L} q_{l} \leq 1}} \log _{2}\left(\operatorname{det}\left(\boldsymbol{I}_{M}+\frac{\rho_{\mathrm{dl}}}{M} \boldsymbol{G}_{\mathrm{dl}}^{\mathrm{H}} \widetilde{\boldsymbol{D}_{\boldsymbol{q}}} \boldsymbol{G}_{\mathrm{dl}}\right)\right)
$$

where $\widetilde{\boldsymbol{D}_{q}}$ is a diagonal matrix of power control co-efficients, whose $l$-th diagonal element is $q_{l}$. It can be readily observed that the Shannon sum spectral effi-

\footnotetext{
[72].

${ }^{9}$ Note that this is also known as the sum spectral efficiency of a multiple access channel
} 
ciency given in (3.7) requires a solution to an optimization problem. Achieving the sum spectral efficiency requires so-called dirty-paper coding (DPC), whose computational complexity grows exponentially with the size of the MU-MIMO system. ${ }^{10}$ Due to this reason, the thesis focuses on linear signal processing approaches (discussed in the following section), whose complexity scales linearly with the system size.

Moreover, it is worth noting that Chapters 4-8 of the thesis directly employ the uplink and downlink MU-MIMO system models presented above. The subsequent section presents the theoretical background necessary on multiuser signal processing techniques on the uplink and downlink of a MU-MIMO system. The signal processing techniques are also a focus of Chapters 4-8 and are used to enhance the sum spectral efficiency of MU-MIMO system with minimal levels of multiuser interference.

\subsection{Multiuser Signal Processing Techniques}

To obtain optimal MU-MIMO performance ${ }^{11}$, complex signal processing techniques must be implemented. For instance, in the uplink, maximum-likelihood (ML) multiuser detection can be used. However, with ML detection, the BS has to search all possible transmitted signal vectors, $\boldsymbol{s}_{\mathrm{ul}}$, and select the optimal one such that

$$
\hat{\boldsymbol{s}}_{\mathrm{ul}}=\arg \min _{\boldsymbol{s}_{\mathrm{ul}} \in \mathcal{S}^{L}}\left\|\boldsymbol{y}_{\mathrm{ul}}-\rho_{\mathrm{ul}}^{\frac{1}{2}} \boldsymbol{G}_{\mathrm{ul}} \boldsymbol{s}_{\mathrm{ul}}\right\|^{2}
$$

where $\mathcal{S}$ is the finite alphabet of $s_{\mathrm{ul}, l}, l=1, \ldots, L$. The optimization problem in (3.8) is a least-squares problem with a finite-alphabet constraint. To implement such an approach, the BS array has to search over $|\mathcal{S}|^{L}$ vectors, where $|\mathcal{S}|$ denotes the cardinality of set $\mathcal{S}$. This exposes the major drawback of the ML detector, its high computational effort. In particular, the computational effort grows exponentially with growing numbers of user terminals in the system.

\footnotetext{
${ }^{10} \mathrm{As}$ a result, it is believed that such types of processing techniques are practically unfeasible for large MU-MIMO systems (the topic of Section 3.5 of the chapter).

${ }^{11}$ Here the term optimal refers to achieving the maximum spectral efficiency.
} 
Alternatively, the BS array can use linear processing techniques via linear combining on the uplink and linear precoding on the downlink to reduce the signal processing complexity at the BS. These techniques are not optimal, however, as the number of BS antennas increase, they are shown to perform near optimally (see $[9,57,74,75]$ and references therein). As a result, the thesis considers the more practical linear processing approaches. Exact details of these techniques is presented in the sections which follow.

\subsubsection{Uplink Linear Combining}

With linear combining at the BS array, the composite received signal at all $M$ antenna elements is separated into $L$ streams by multiplication with an $L \times M$ linear detector matrix in $\boldsymbol{T}=\left[\boldsymbol{t}_{1}, \ldots, \boldsymbol{t}_{M}\right]$, such that the combined signal can be written as

$$
\boldsymbol{r}_{\mathrm{ul}}=\boldsymbol{T} \boldsymbol{y}_{\mathrm{ul}}=\rho_{\mathrm{ul}}^{\frac{1}{2}} \boldsymbol{T} \boldsymbol{G}_{\mathrm{ul}} \boldsymbol{s}_{\mathrm{ul}}+\boldsymbol{T} \boldsymbol{n}_{\mathrm{ul}}
$$

If each uplink data stream is decoded independently, a computational effort of order $L|\mathcal{S}|$ is observed in comparison to ML decoding. As a result, the stream transmitted from terminal $l$ corresponds to the $l$-th element of $\boldsymbol{r}_{\mathrm{ul}}$, and is used to decode $s_{\mathrm{ul}, l}$. This can be written as

$$
r_{\mathrm{ul}, l}=\rho_{\mathrm{ul}}^{\frac{1}{2}} \boldsymbol{t}_{l} \boldsymbol{g}_{\mathrm{ul}, l} s_{\mathrm{ul}, l}+\rho_{\mathrm{ul}}^{\frac{1}{2}} \sum_{\substack{k=1 \\ k \neq l}}^{L} \boldsymbol{t}_{l} \boldsymbol{g}_{\mathrm{ul}, k} s_{\mathrm{ul}, k}+\boldsymbol{t}_{l} \boldsymbol{n}_{\mathrm{ul}},
$$

where the first term on the right hand side of the equal sign denotes the desired signal, the second term denotes multiuser interference and the third term denotes additive Gaussian noise. Moreover, $\boldsymbol{t}_{l}$ denotes the $l$-th column of $\boldsymbol{T}$.

From this, the signal-to-interference-plus-noise-ratio (SINR) for the $l$-th terminal can be written as

$$
\operatorname{SINR}_{\mathrm{ul}, l}=\frac{\rho_{\mathrm{ul}}\left|\boldsymbol{t}_{l} \boldsymbol{g}_{\mathrm{ul}, l}\right|^{2}}{\sigma_{\mathrm{ul}, l}^{2}|| \boldsymbol{t}_{l} \|^{2}+\rho_{\mathrm{ul}} \sum_{\substack{k=1 \\ k \neq l}}^{L}\left|\boldsymbol{t}_{l} \boldsymbol{g}_{\mathrm{ul}, k}\right|^{2}} .
$$


Equivalently, the uplink spectral efficiency for terminal $l$ can be written as

$$
\mathrm{R}_{\mathrm{ul}, l}=\log _{2}\left(1+\mathrm{SINR}_{\mathrm{ul}, l}\right) .
$$

As such, the uplink ergodic sum spectral efficiency over all $L$ terminals is given by

$$
\mathbb{E}\left[\mathrm{R}_{\mathrm{ul}, \text { sum }}\right]=\mathbb{E}\left[\sum_{l=1}^{L} \mathrm{R}_{\mathrm{ul}, l}\right] .
$$

Remark 3.4.1. Note that the SINR is a metric to analyze (and evaluate) the instantaneous performance for a given terminal, in contrast to the ergodic sum spectral efficiency, which is a metric to analyze (and evaluate) the performance of the entire MU-MIMO system. It is also worth noting that both the SINR and the ergodic sum spectral efficiency are sensitive to the type of processing employed in $\boldsymbol{T}$. In the following, the three linear combiners used throughout the thesis (maximum-ratio combining (MRC), zero-forcing combining (ZF) and minimum-mean-squared-error combining (MMSE)) are reviewed.

\section{Maximum-Ratio Combining}

With MRC, the BS aims to maximize the received signal power on each stream transmitted from the terminals, neglecting the effects of multiuser interference. From (3.10), the $l$-th column of the MRC receiver matrix, $\boldsymbol{T}$, is given by

$$
\boldsymbol{t}_{l}=\arg \max _{\boldsymbol{t}_{l}} \frac{\text { power(desired signal) }}{\text { power(additive noise) }}=\arg \max _{\boldsymbol{t}_{l}} \frac{\rho_{\mathrm{ul}} \beta_{l}\left|\boldsymbol{t}_{l} \boldsymbol{h}_{\mathrm{ul}, l}\right|^{2}}{\sigma_{\mathrm{ul}, l}^{2}|| \boldsymbol{t}_{l}||^{2}} .
$$

Since

$$
\frac{\rho_{\mathrm{ul}} \beta_{l}\left|\boldsymbol{t}_{l} \boldsymbol{h}_{\mathrm{ul}, l}\right|^{2}}{\sigma_{\mathrm{ul}, l}^{2}|| \boldsymbol{t}_{l}||^{2}} \leq \frac{\rho_{\mathrm{ul}} \beta_{l}\left\|\boldsymbol{t}_{l}||^{2}\right\| \boldsymbol{h}_{\mathrm{ul}, l} \|^{2}}{\sigma_{\mathrm{ul}, l}^{2}|| \boldsymbol{t}_{l} \|^{2}}=\rho_{\mathrm{ul}} \beta_{l}\left\|\boldsymbol{h}_{\mathrm{ul}, l}\right\|^{2},
$$

with the equality holding when $\boldsymbol{t}_{l}=\boldsymbol{h}_{\mathrm{ul}, l}^{\mathrm{H}}$, it follows that for MRC combiner, for the stream coming from terminal $l$ is given by

$$
\boldsymbol{t}_{l}=\boldsymbol{h}_{\mathrm{ul}, l}^{\mathrm{H}} \text {. }
$$


Substituting $\boldsymbol{t}_{l}$ from (3.16) into (3.11), the received SINR for terminal $l$ with MRC uplink processing is given by

$$
\operatorname{SINR}_{\mathrm{ul}, l}=\frac{\rho_{\mathrm{ul}} \beta_{l}\left\|\boldsymbol{h}_{\mathrm{ul}, l}\right\|^{4}}{\sigma_{\mathrm{ul}, l}^{2}\left\|\boldsymbol{h}_{\mathrm{ul}, l}\right\|^{2}+\rho_{\mathrm{ul}} \sum_{\substack{k=1 \\ k \neq l}}^{L} \beta_{k}\left|\boldsymbol{h}_{\mathrm{ul}, l}^{\mathrm{H}} \boldsymbol{h}_{\mathrm{ul}, k}\right|^{2}} .
$$

Remark 3.4.2. The MRC processor has several advantages, as well as disadvantages. The advantage is that the uplink signal processing becomes extremely simple, since the BS array multiplies the received signals by the conjugate channel responses, and is then able to detect each data stream separately. As a result of this, MRC is useful because it allows operation in a distributed fashion, independently at each antenna element [11]. Furthermore, at low operating SNRs, the MRC SINR for terminal $l$ is primarily driven by $\rho_{\mathrm{ul}} \beta_{l}\left\|\boldsymbol{h}_{l}\right\|^{2}$. This implies that at low operating SNRs, MRC can achieve the same array gain, as in the case of a single-user system [76]. On the other hand, the disadvantage is that MRC neglects the effects of multiuser interference and thus is expected to perform poorly in interference limited scenarios, such as at high operating SNRs. This can be seen by taking the limit as $\rho_{\mathrm{ul}} \rightarrow \infty$ and observing that the MRC SINR collapses to

$$
\lim _{\rho_{\mathrm{ul}} \rightarrow \infty}\left\{\operatorname{SINR}_{\mathrm{ul}, l}\right\}=\frac{\beta_{l}\left\|\boldsymbol{h}_{\mathrm{ul}, l}\right\|^{4}}{\beta_{k} \sum_{\substack{k=1 \\ k \neq l}}^{L}\left|\boldsymbol{h}_{\mathrm{ul}, l}^{\mathrm{H}} \boldsymbol{h}_{\mathrm{ul}, k}\right|^{2}},
$$

where it is upper bounded by a constant value (with respect to $\rho_{\mathrm{ul}}$ ) when $\rho_{\mathrm{ul}}$ is large. Performance analysis with MRC is presented in Chapters 6 and 8 of the thesis with i.i.d. Rayleigh and unequally correlated Ricean fading channels, respectively.

\section{Zero-Forcing Combining}

In contrast to MRC, the ZF combiner considers the effects of multiuser interference while neglecting the effects of additive Gaussian noise. In fact, ZF combining completely nulls the multiuser interference by projecting each data 
stream onto the orthogonal complement of the inter-user interference. Mathematically, the $l$-th column of the ZF combiner matrix satisfies $\boldsymbol{t}_{l} \boldsymbol{g}_{\mathrm{ul}, l} \neq 0$ and $\boldsymbol{t}_{l} \boldsymbol{g}_{\mathrm{ul}, k}=0, \forall k \neq l$. The ZF combining matrix which satisfies the above constraint for all $l$, is the pseudo-inverse of the composite instantaneous small-scale fading channel matrix. With ZF, one can rewrite (3.9) as

$$
\begin{aligned}
\boldsymbol{r}_{\mathrm{ul}} & =\left(\boldsymbol{G}_{\mathrm{ul}}^{\mathrm{H}} \boldsymbol{G}_{\mathrm{ul}}\right)^{-1} \boldsymbol{G}_{\mathrm{ul}}^{\mathrm{H}} \boldsymbol{y}_{\mathrm{ul}} \\
& =\rho_{\mathrm{ul}}^{\frac{1}{2}}\left(\boldsymbol{G}_{\mathrm{ul}}^{\mathrm{H}} \boldsymbol{G}_{\mathrm{ul}}\right)^{-1} \boldsymbol{G}_{\mathrm{ul}}^{\mathrm{H}} \boldsymbol{G}_{\mathrm{ul}} \boldsymbol{s}_{\mathrm{ul}}+\left(\boldsymbol{G}_{\mathrm{ul}}^{\mathrm{H}} \boldsymbol{G}_{\mathrm{ul}}\right)^{-1} \boldsymbol{G}_{\mathrm{ul}}^{\mathrm{H}} \boldsymbol{n}_{\mathrm{ul}} \\
& \stackrel{(a)}{=} \rho_{\mathrm{ul}}^{\frac{1}{2}} \boldsymbol{s}_{\mathrm{ul}}+\left(\boldsymbol{G}_{\mathrm{ul}}^{\mathrm{H}} \boldsymbol{G}_{\mathrm{ul}}\right)^{-1} \boldsymbol{G}_{\mathrm{ul}}^{\mathrm{H}} \boldsymbol{n}_{\mathrm{ul}},
\end{aligned}
$$

where $(a)$ is a result of $\left(\boldsymbol{G}_{\mathrm{ul}}^{\mathrm{H}} \boldsymbol{G}_{\mathrm{ul}}\right)^{-1} \boldsymbol{G}_{\mathrm{ul}}^{\mathrm{H}} \boldsymbol{G}_{\mathrm{ul}}=\boldsymbol{I}_{L}$. It is worth noting that the ZF processor requires that $M \geq L$, such that the matrix $\boldsymbol{G}_{\mathrm{ul}}^{\mathrm{H}} \boldsymbol{G}_{\mathrm{ul}}$ is invertible. ${ }^{12}$ As a result of this, one can observe that the $l$-th data stream (element) of $\boldsymbol{r}_{\mathrm{ul}}$ in (3.19) is free from multiuser interference, where the $l$-th stream used to detect $s_{\mathrm{ul}, \mathrm{l}}$ is given by

$$
r_{\mathrm{ul}, \mathrm{l}}=\rho_{\mathrm{ul}}^{\frac{1}{2}} s_{\mathrm{ul}, \mathrm{l}}+\tilde{n}_{\mathrm{ul}, l}
$$

where $\tilde{n}_{\mathrm{ul}, l}$ denotes the $l$-th element of $\left(\boldsymbol{G}_{\mathrm{ul}}^{\mathrm{H}} \boldsymbol{G}_{\mathrm{ul}}\right)^{-1} \boldsymbol{G}_{\mathrm{ul}}^{\mathrm{H}} \boldsymbol{n}_{\mathrm{ul}}$. Due to the complete cancellation of multiuser interference, the instantaneous per-terminal SINR translates into a per-terminal SNR. For the $l$-th terminal, the ZF SNR is given by

$$
\mathrm{SNR}_{\mathrm{ul}, l}=\frac{\rho_{\mathrm{ul}} \beta_{l}}{\sigma_{\mathrm{ul}, \mathrm{l}}^{2}\left[\left(\boldsymbol{H}_{\mathrm{ul}}^{\mathrm{H}} \boldsymbol{H}_{\mathrm{ul}}\right)^{-1}\right]_{l, l}}
$$

where $\left[(\boldsymbol{A})^{-1}\right]_{l, l}$ denotes the $(l, l)$-th element of $(\boldsymbol{A})^{-1}$.

Remark 3.4.3. The $\mathrm{ZF}$ combiner is an ideal candidate for interference limited cellular systems, due to its ability to null inter-user interference. As a result of this, the SNR for terminal $l$ can be made as high as desirable by virtue of increasing the link SNR. Pushing the link SNR to an extreme, i.e., letting SNR $\rightarrow \infty$ will yield an infinitely high ZF SNR for terminal $l$. This is straightforward to observe from (3.21). On the contrary, since ZF processing

\footnotetext{
${ }^{12}$ The inverse does not exist for systems which violate this restriction due to rank deficiency.
} 
neglects the effects of additive Gaussian noise, it operates poorly under noise limited scenarios, i.e., with low link SNR levels. Moreover, if the channel is not well conditioned (partly controlled by the size of $M$ in comparison to $L$ ), then performing the pseudo-inverse tends to significantly amplify the instantaneous noise power, leading to poor performance. In comparison to MRC, the ZF combiner has a high computational effort, due to the involvement of a pseudo-inverse of the channel gain matrix. This is particularly disadvantageous for large MU-MIMO systems, where the size of the inverse scales linearly with the number of terminals in the system. It is also to be noted that the calculation of a particular inverse scales as the cube of its size. Hence, even a modest increase in the number of terminals can lead to a significant growth in the computational demand. Several alternatives are considered to overcome the effects of this complexity burden by decomposing the inverse with finite series expansion techniques. One example is the Neumann series (see e.g., $[57,77,78]$ and references therein), where the inverse is approximated with polynomials having a low order. Chapter 7 in the thesis analyzes the performance of unequally correlated Rayleigh fading channels with ZF processing using Neumann series expansions. Also, Chapter 6 of the thesis presents simplified uplink analysis with ZF combining over i.i.d. Rayleigh fading channels.

\section{Minimum-Mean-Squared-Error Combining}

Unlike both the MRC and ZF combining solutions, the linear minimum-meansquared-error (MMSE) combiner aims to minimize the mean-squared error between the estimate $\boldsymbol{T} \boldsymbol{y}_{\mathrm{ul}}$ and the transmitted signal, $\boldsymbol{s}_{\mathrm{ul}}$. More particularly, the $L \times M$ MMSE combiner matrix, $\boldsymbol{T}$, is designed by

$$
\begin{aligned}
\boldsymbol{T} & =\arg \min _{\boldsymbol{T}} \mathbb{E}\left[\| \boldsymbol{T} \boldsymbol{y}_{\mathrm{ul}}-\boldsymbol{s}_{\mathrm{ul}}||^{2}\right] \\
& =\arg \min _{\boldsymbol{T}} \sum_{l=1}^{L} \mathbb{E}\left[\left|\boldsymbol{t}_{l} \boldsymbol{y}_{\mathrm{ul}}-s_{\mathrm{ul}, l}\right|^{2}\right] .
\end{aligned}
$$


Thus, the $l$-th column of $\boldsymbol{T}$ is given by

$$
\begin{aligned}
\boldsymbol{t}_{l} & =\arg \min _{\boldsymbol{t}_{l}} \mathbb{E}\left[\left|\boldsymbol{t}_{l} \boldsymbol{y}_{\mathrm{ul}}-s_{\mathrm{ul}, l}\right|^{2}\right] \\
& =\rho_{\mathrm{ul}}^{\frac{1}{2}}\left(\rho_{\mathrm{ul}} \boldsymbol{G}_{\mathrm{ul}} \boldsymbol{G}_{\mathrm{ul}}^{\mathrm{H}}+\boldsymbol{I}_{M}\right)^{-1} \boldsymbol{g}_{\mathrm{ul}, l} .
\end{aligned}
$$

Remark 3.4.4. As far as linear processors are concerned, the MMSE combiner provides the ultimate solution in-terms of the trade-off between the maximization of signal power, while controlling noise and interference powers. Further inspection of (3.23) yields that at high link SNRs, i.e., high $\rho_{\mathrm{ul}}$, with a fixed $\sigma_{\mathrm{ul}, l}^{2}$ for all $L$ terminals, the performance of ZF processing approaches that of MMSE processing, while at low link SNRs, MRC performs just as well as MMSE. Hence, depending on the operating SNR regime, MMSE combining can be thought of as tuning between MRC and ZF combining at low and high SNRs. Furthermore, substituting (3.23) into (3.10), the received SINR for the MMSE combiner can be written as

$$
\mathrm{SINR}_{\mathrm{ul}, l}=\rho_{\mathrm{ul}} \beta_{l}\left\{\boldsymbol{h}_{\mathrm{ul}, l}^{\mathrm{H}}\left(\rho_{\mathrm{ul}} \beta_{k} \sum_{\substack{k=1 \\ k \neq l}}^{L} \boldsymbol{h}_{\mathrm{ul}, k} \boldsymbol{h}_{\mathrm{ul}, k}^{\mathrm{H}}+\boldsymbol{I}_{M}\right)^{-1} \boldsymbol{h}_{\mathrm{ul}, l}\right\} .
$$

Remark 3.4.5. Using the expressions in (3.11) and (3.12), the corresponding per-terminal spectral efficiency and the ergodic sum spectral efficiency can be evaluated for any uplink processing type. In the following section, the equivalent linear processing approaches on the downlink are outlined.

\subsubsection{Downlink Linear Precoding}

In the downlink, virtually the opposite happens with linear precoding techniques helping the service array at the BS to serve multiple user terminals in the same time-frequency resource. The downlink signal, $\boldsymbol{s}_{\mathrm{dl}}$, transmitted from the $M$ elements is a linear combination of the symbols intended for the $L$ terminals. Let $\tilde{s}_{\mathrm{dl}, l}$ be the message bearing data symbol intended for terminal $l$, such that 
$\mathbb{E}\left[\left|\tilde{s}_{\mathrm{dl}, l}\right|^{2}\right]=1$. Hence, the linearly precoded signal at the BS array is given by

$$
\boldsymbol{s}_{\mathrm{dl}}=\eta^{\frac{1}{2}} \boldsymbol{W} \tilde{\boldsymbol{s}}_{\mathrm{dl}},
$$

where $\tilde{\boldsymbol{s}}_{\mathrm{dl}}=\left[\tilde{s}_{\mathrm{dl}, 1}, \ldots, \tilde{s}_{\mathrm{dl}, L}\right]^{\mathrm{T}}$ is the $L \times 1$ vector of data symbols, $\boldsymbol{W}$ is the $M \times L$ downlink precoding matrix and $\eta$ is the precoder normalization parameter chosen to satisfy the overall power constraint of $\mathbb{E}\left[\| s_{\mathrm{dl}}||^{2}\right]=1$. Therefore,

$$
\eta=\frac{1}{\mathbb{E}\left[\operatorname{tr}\left[\boldsymbol{W} \boldsymbol{W}^{\mathrm{H}}\right]\right]} .
$$

Remark 3.4.6. It is worth noting that throughout the thesis, the matrix normalization described above is used. An alternative way to normalize the power in each downlink precoder is by normalizing each column of the precoding matrix. Both approaches are widely accepted in the MU-MIMO literature, yielding similar results $[9,57,59,79-83]$.

Substituting the precoded signal expression in (3.25) into (3.5), for a particular type of linear precoder, $\boldsymbol{W}$, one can obtain the received signal for terminal $l$ as

$$
\begin{aligned}
r_{\mathrm{dl}, l} & =\rho_{\mathrm{dl}}^{\frac{1}{2}} \eta^{\frac{1}{2}} \beta_{l} \boldsymbol{h}_{\mathrm{dl}, l}^{\mathrm{H}} \boldsymbol{W} \tilde{\boldsymbol{s}}_{\mathrm{dl}}+n_{\mathrm{dl}, l} \\
& =\rho_{\mathrm{dl}}^{\frac{1}{2}} \eta^{\frac{1}{2}} \beta_{l} \boldsymbol{h}_{\mathrm{dl}, l}^{\mathrm{H}} \boldsymbol{w}_{l} \tilde{s}_{\mathrm{dl}, l}+\rho_{\mathrm{dl}}^{\frac{1}{2}} \eta^{\frac{1}{2}} \beta_{l} \sum_{\substack{k=1 \\
k \neq l}}^{L} \boldsymbol{h}_{\mathrm{dl}, l}^{\mathrm{H}} \boldsymbol{w}_{k} \tilde{s}_{\mathrm{dl}, k}+n_{\mathrm{dl}, l} .
\end{aligned}
$$

Therefore, the SINR for terminal $l$ as a result of transmission from the BS array to the $l$-th terminal can be written as

$$
\operatorname{SINR}_{\mathrm{dl}, l}=\frac{\rho_{\mathrm{dl}} \eta \beta_{l}\left|\boldsymbol{h}_{\mathrm{d} l, l}^{\mathrm{H}} \boldsymbol{w}_{l}\right|^{2}}{\sigma_{\mathrm{dl}, l}^{2}+\rho_{\mathrm{dl}} \eta \beta_{l} \sum_{\substack{k=1 \\ k \neq l}}^{L}\left|\boldsymbol{h}_{\mathrm{d} 1, l}^{\mathrm{H}} \boldsymbol{w}_{k}\right|^{2}} .
$$

Three conventional linear precoders are often employed in MU-MIMO studies. These are matched-filter (MF) precoding (also known as conjugate beamforming), ZF precoding and regularized ZF (RZF) precoding (for single-stream 
transmission, also known as MMSE precoding, signal-to-leakage-plus-noise-ratio precoding and transmit Wiener filter). On the downlink, these precoders perform the equivalent activity as linear combiners do on the uplink. Due to this reason, these precoders have similar operational meanings and properties as the MRC, ZF, and MMSE receivers, respectively. Due to this reason, the final structure of each precoding type is provided in the following expression. ${ }^{13}$

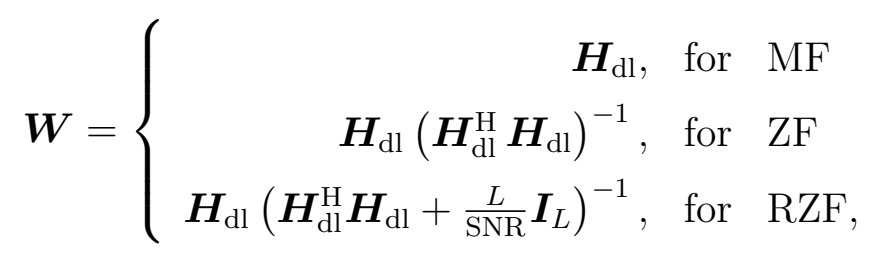

From (3.29), by taking the $l$-th column of $\boldsymbol{W}$, the SINR for the $l$-th terminal in (3.28) can be written for different processing types. The downlink SINR for terminal $l$ with a particular type of processor can be translated into an instantaneous downlink spectral efficiency for terminal $l$ via the classical Shannon equation in (3.12). From then, the ergodic sum spectral efficiency can be computed by following (3.13).

Figs. 3.3 and 3.4 demonstrate the uplink and downlink ergodic sum spectral efficiency with the above mentioned processing approaches, as a function of the operating SNR $\left(\rho_{\mathrm{ul}}\right.$ for uplink and $\rho_{\mathrm{dl}}$ for the downlink, assuming $\sigma_{\mathrm{dl}}^{2}=\sigma_{\mathrm{ul}}^{2}=1$. At the BS, $M=6$ transmit antenna elements are considered communicating with $L=4$ terminals. In order not to obfuscate the performance trends, $\beta_{l}=1$, $\forall l=1, \ldots, L$. The noise limited and interference limited performance of the uplink and downlink processors is clearly visible with the MMSE and RZF processors providing the tuning between MRC/MF and ZF at low and high link SNRs.

In what follows, a discussion on the expected per-terminal SINR is presented for both uplink and downlink processing techniques.

\footnotetext{
${ }^{13}$ One can equally chose to precode over $\boldsymbol{G}_{\mathrm{dl}}$, instead of $\boldsymbol{H}_{\mathrm{dl}}$.
} 


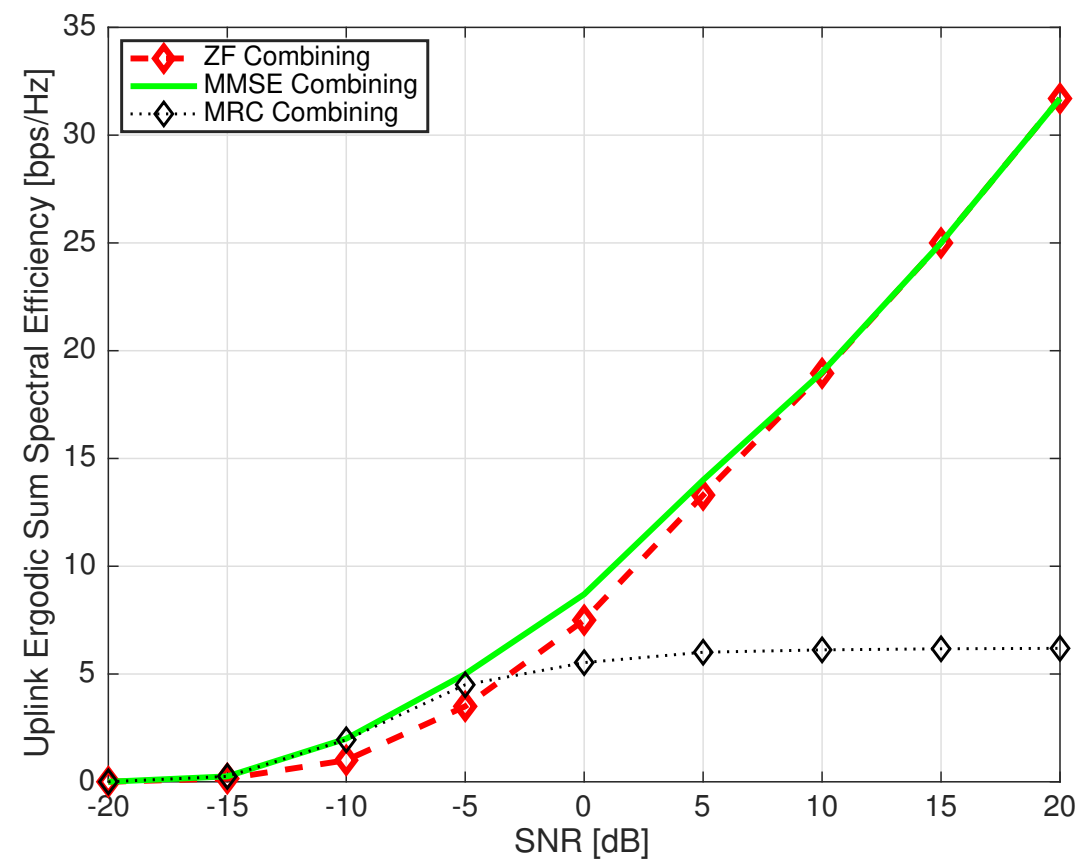

Figure 3.3: Ergodic sum spectral efficiency performance of MRC, ZF and MMSE uplink combiners as a function of increasing link SNRs.

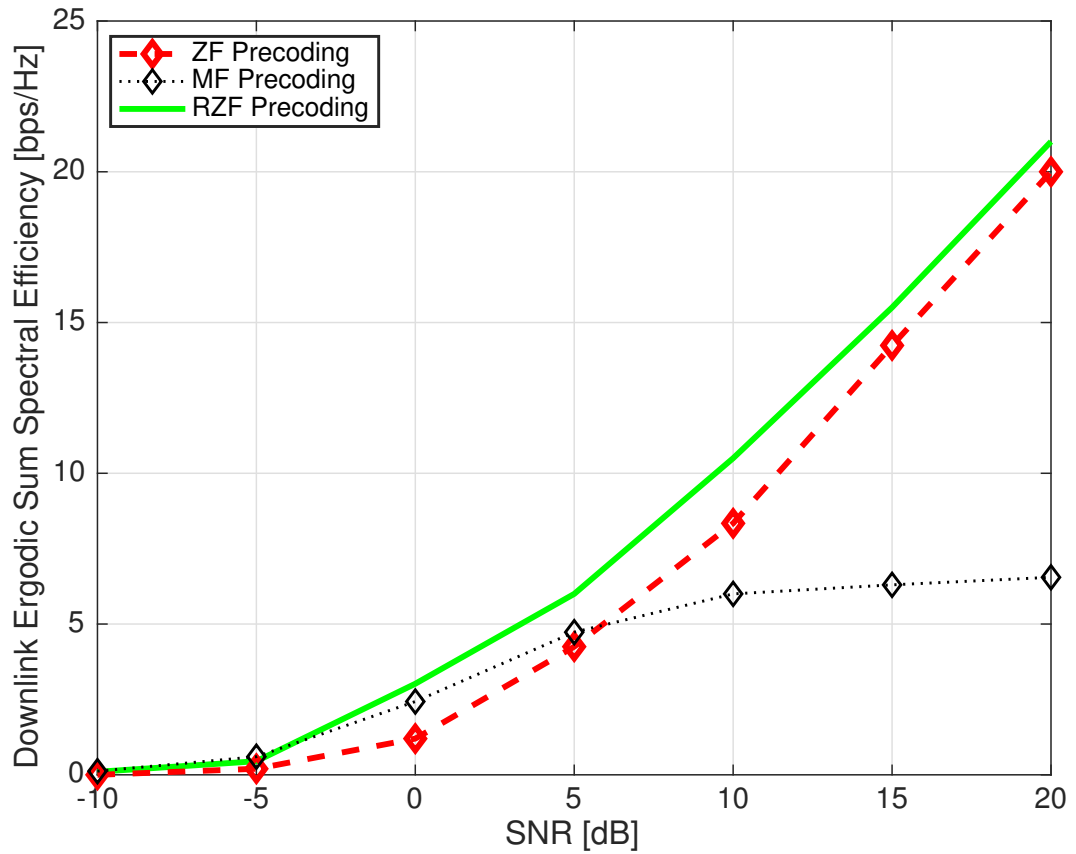

Figure 3.4: Ergodic sum spectral efficiency performance of MF, ZF and RZF downlink precoding as a function of increasing link SNRs. 


\subsubsection{Expected Per-Terminal SINR Approximation}

Throughout the thesis, for both uplink and downlink MU-MIMO systems, the average (expected) performance of a given user terminal is analyzed with a commonly employed first-order delta approximation. The aim of this section is to justify the validity of this approximation and identify the conditions required for the approximation to be extremely tight. For ease of exposition, the downlink SINR for terminal $l$ is considered, however, the discussion equally applies to uplink MU-MIMO systems, since the SINR takes on similar random quantities in the numerator and the denominator. The SINR of terminal $l$, given a linear precoder $\boldsymbol{W}$ can be expressed as in (3.28). The expected per-terminal SINR requires an expectation of (3.28). In general, exact evaluation of

$$
\mathbb{E}\left[\mathrm{SINR}_{\mathrm{dl}, l}\right]=\mathbb{E}\left[\frac{\rho_{\mathrm{dl}} \eta \beta_{l}\left|\boldsymbol{h}_{\mathrm{dl}, l}^{\mathrm{H}} \boldsymbol{w}_{l}\right|^{2}}{\sigma_{\mathrm{dl}, l}^{2}+\rho_{\mathrm{dl}} \eta \beta_{l} \sum_{\substack{k=1 \\ k \neq l}}^{L}\left|\boldsymbol{h}_{\mathrm{dl}, l}^{\mathrm{H}} \boldsymbol{w}_{k}\right|^{2}}\right],
$$

is extremely cumbersome due a multitude of factors, the biggest one being that the expectation is performed on a function containing a ratio of random variables. Hence, communication researchers often employ the first-order Delta method approximation, which allows one to analyze the expectation in the numerator and denominator separately. The approximation allows the expected SINR for terminal $l$ to be written as

$$
\mathbb{E}\left[\mathrm{SINR}_{\mathrm{dl}, l}\right] \approx \frac{\rho_{\mathrm{dl}} \tilde{\eta} \beta_{l} \mathbb{E}\left[\left|\boldsymbol{h}_{\mathrm{dl}, l}^{\mathrm{H}} \boldsymbol{w}_{l}\right|^{2}\right]}{\sigma_{\mathrm{dl}, l}^{2}+\rho_{\mathrm{dl}} \tilde{\eta} \beta_{l} \sum_{\substack{k=1 \\ k \neq l}}^{L} \mathbb{E}\left[\left|\boldsymbol{h}_{\mathrm{dl}, l}^{\mathrm{H}} \boldsymbol{w}_{k}\right|^{2}\right]},
$$

where $\tilde{\eta}=\mathbb{E}[\eta]$.

Remark 3.4.7. The approximation in (3.31) is of the form $\mathbb{E}\left[\frac{X}{Y}\right] \approx \frac{\mathbb{E}[X]}{\mathbb{E}[Y]}$, where $X$ and $Y$ both contain standard quadratic forms $[68,80,83-88]$. The accuracy of such approximations relies on $Y$ having a small variance relative to its mean value. This can be seen by applying a multivariate Taylor series expansion of $\frac{X}{Y}$ around $\frac{\mathbb{E}[X]}{\mathbb{E}[Y]}$, as shown in the analysis methodology of [68]. In particular, the quadratic forms in (3.31) are well suited to this approximation as 
$M$ and $L$ start to increase, where the approximation is shown to be extremely tight. This is due to the quadratic forms averaging their respective individual components, minimizing their variance relative to their mean values. For further discussion, the more interested reader is refereed to $[68,88]$, where a detailed mathematical proof of the approximation accuracy can be found.

Following from (3.31), the ergodic sum spectral efficiency for all $L$ terminals can be approximated by

$$
\mathbb{E}\left[\mathrm{R}_{\mathrm{dl}, \mathrm{sum}}\right]=\mathbb{E}\left[\sum_{l=1}^{L} \log _{2}\left(1+\operatorname{SINR}_{\mathrm{dl}, l}\right)\right] \approx \sum_{l=1}^{L} \log _{2}\left(1+\mathbb{E}\left[\operatorname{SINR}_{\mathrm{dl}, l}\right]\right)
$$

Remark 3.4.8. Note that (3.32) leads to an approximation rather than an upper bound vis Jensen's inequality, as the value of $\mathbb{E}\left[\mathrm{SINR}_{\mathrm{dl}, l}\right]$ is itself an approximation $[81,85]$. Moreover, the summation over $L$ terminals in the service array's coverage area takes care of the fact that $\mathbb{E}\left[\mathrm{SINR}_{\mathrm{dl}, l}\right]$ could be different depending on the index $l$, due to the relative differences in the physical location of each terminal, causing further differences in the level of geometric attenuation and log-normal shadow-fading.

The above mentioned approximations are utilized in Chapters 4-8 of the thesis, for analysis of different multiuser transceiver structures over heterogeneous fading channels. This concludes the conventional MU-MIMO background required for the thesis, leading to large MU-MIMO systems.

\subsection{Large Multiuser MIMO Systems}

Since the landmark work by Marzetta in 2010, large MU-MIMO systems have emerged as a key solution to meet the spectral and energy efficiency demands of future cellular networks $[9,57,71]$. Increasing the number of service antennas by an order of magnitude, these systems push conventional MU-MIMO systems to an extreme.

Naturally, all the previously presented background on conventional MUMIMO operation and signal processing aspect is also directly applicable to large 
MU-MIMO. The benefits of growing the number of BS antennas relative to the number of active terminals include: (1) Greater selectivity in transmitting and receiving data streams, in turn leading to greater spectral efficiency; (2) Reduction in the required radiated power, allowing for effective power control providing uniformly good service throughout the service array's coverage area; (3) Even further simplification in the signal processing at the BS. ${ }^{14}$ Furthermore, letting the number of service antennas grow without bound removes the effects of fastfading and multiuser interference, as the propagation channels become mutually pairwise orthogonal. ${ }^{15}$

The effects of increasing numbers of service antennas on the composite channel matrix, sum spectral efficiency, and linear processing techniques are discussed below, where single antenna terminals are assumed for notational simplicity.

\subsubsection{Convergence to Favorable Propagation Conditions}

In this subsection, the impact of an indefinite number of service antennas on the channel correlation matrix is demonstrated. Again for ease of exposition, i.i.d. Rayleigh fading channels are assumed. Later, in Chapter 8 of the thesis, the performance of an uplink MRC MU-MIMO system is analyzed using the principles described below, over unequally correlated Ricean fading channels. In particular, the typical large MU-MIMO literature examines the behavior of $\frac{1}{M} \boldsymbol{G}_{\mathrm{dl}}^{\mathrm{H}} \boldsymbol{G}_{\mathrm{dl}}$ is demonstrated, where the $\boldsymbol{g}_{l}^{\mathrm{H}}$ is the $1 \times M$ downlink propagation channel from the service array to the $l$-th terminal. Now, as the number of service antennas, $M \rightarrow \infty$, with a fixed number of terminals in $L$, it is straightforward to observe

\footnotetext{
${ }^{14}$ As a result of simplifications in baseband signal processing, large MU-MIMO systems also simplify media access control layer design.

${ }^{15}$ In the limit of an unlimited number of BS antennas serving a finite number of terminals, the only remaining impairment results from imperfect CSI through the re-use of pilot sequences in multicellular networks. For TDD systems, this corresponds to the BSs uplink channel estimate, through channel reciprocity, being corrupted by terminals in adjacent cells using the same pilot sequence. In FDD systems, this corresponds to the BS sending out duplicates of the (limited) number of pilot sequences, as the number of downlink pilot sequences required scales with the number of service antennas.
} 
that $\frac{1}{M} \boldsymbol{G}_{\mathrm{dl}}^{\mathrm{H}} \boldsymbol{G}_{\mathrm{dl}}$ approaches a diagonal matrix. Mathematically speaking,

$$
\lim _{M \rightarrow \infty}\left\{\frac{1}{M} \boldsymbol{G}_{\mathrm{dl}}^{\mathrm{H}} \boldsymbol{G}_{\mathrm{dl}}\right\}=\lim _{M \rightarrow \infty}\left\{\boldsymbol{D}^{\frac{1}{2}} \frac{\boldsymbol{H}_{\mathrm{dl}}^{\mathrm{H}} \boldsymbol{H}_{\mathrm{dl}}}{M} \boldsymbol{D}^{\frac{1}{2}}\right\}=\boldsymbol{D}
$$

This is a result of each $1 \times M$ propagation channel becoming asymptotically pairwise orthogonal $[9,89]$. Here, $\boldsymbol{D}=\operatorname{diag}\left(\beta_{1}, \ldots, \beta_{L}\right)$ is the $L \times L$ diagonal matrix of link gains for each terminal. In the large MU-MIMO literature, this has been famously coined as convergence to favorable propagation.

Remark 3.5.1. Numerous physical implications can be drawn from (3.33). The most obvious observation is that channel orthogonality is a result of having a large number service antennas. As a result of this, propagation channels to multiple terminals become extremely diverse, facilitating maximum spatial separation of the transmitted data streams to different terminals. Moreover, under favorable propagation conditions, the effects of small-scale fading is seen to vanish over the service array and each communication link is only governed by its large-scale fading co-efficient. ${ }^{16}$ If such propagation conditions can be realized in practice, multiuser scheduling, transmit power control, and multiuser processing can be performed only considering large-scale fading co-efficients, which was introduced earlier to vary at a different time scale than the smallscale fading. This further reduces the total overheads and signal processing complexity in the design of such systems.

In the following subsection, the implications of favorable propagation on the sum spectral efficiency is introduced.

\subsubsection{Impact on Sum Spectral Efficiency}

The impact of substituting the result presented in (3.33) into the downlink sum spectral efficiency expression presented earlier in (3.7) yields a number of mathematically exact conclusions. For exposition purposes, the downlink case is analyzed here, however, the same conclusions also hold for uplink systems.

\footnotetext{
${ }^{16}$ Naturally, the rate at which favorable propagation occurs is ultimately governed by the underlying propagation characteristics.
} 
Since $\frac{1}{M} \boldsymbol{G}_{\mathrm{dl}}^{\mathrm{H}} \boldsymbol{G}_{\mathrm{dl}} \rightarrow \boldsymbol{I}_{L}$, as $M \rightarrow \infty$, the spatial multiplexing gains of the system can be maximized and thus the sum spectral efficiency achieves the capacity (optimal sum spectral efficiency). This can be observed by taking the limit of (3.7), as $M \rightarrow \infty$. This results in

$$
\begin{aligned}
\lim _{M \rightarrow \infty}\left\{\mathrm{R}_{\mathrm{dl}, \text { sum }}\right\} & =\lim _{M \rightarrow \infty}\left\{\log _{2}\left(\operatorname{det}\left(\boldsymbol{I}_{M}+\frac{\rho_{\mathrm{dl}}}{M} \boldsymbol{G}_{\mathrm{dl}}^{\mathrm{H}} \boldsymbol{G}_{\mathrm{dl}}\right)\right)\right\} \\
& =\log _{2}\left(\operatorname{det}\left(\boldsymbol{I}_{L}+\rho_{\mathrm{dl}} \boldsymbol{D}\right)\right) \\
& =\sum_{l=1}^{L} \log _{2}\left(1+\rho_{\mathrm{dl}} \beta_{l}\right) .
\end{aligned}
$$

Remark 3.5.2. The expression in (3.34) is seen to scale with the number of terminals in the system. With the absence of fast-fading, this suggests that the sum spectral efficiency can be broken down into $L$ parallel additive Gaussian noise channels, as a function of the link SNR and the large-scale fading parameter for a given terminal. As small-scale fading has no part to play in (3.34), the sum spectral efficiency only undergoes variations induced by large-scale fading by virtue of the link gain and link SNR. This phenomena is known as channel hardening in the large MU-MIMO literature.

\subsubsection{Space-Constrained MU-MIMO Systems}

The large number of service antennas that large antenna arrays advocate implies that the physical space dedicated to locate the antenna elements must also grow, assuming they are deployed with fixed inter-element spacings $[57,89,90]$. Nevertheless, a limit in the maximum number of antennas which could be deployed is to be expected due to physical size restrictions on the service array. For instance, consider an array with $M=128$ serving elements operating at the typical carrier frequency of $2.6 \mathrm{GHz}$, where the corresponding wavelength, $\lambda_{c}=11.5 \mathrm{~cm}$. If each antenna was to be deployed with an inter-element spacing of $d_{s} \geq 0.5 \lambda_{c}$ to avoid the harmful effects of spatial correlation and antenna coupling, placing the elements in a ULA would seem impractical, since the size of the resultant array would be at least $M \lambda_{c} / 2=(128)(0.115 \mathrm{~m}) / 2=7.36 \mathrm{~m}$. 
Naturally, such array sizes are larger than the physical structures currently being employed in cellular systems of today, further complicating their deployments.

The above discussion has been the major driving force for research into space-constrained antenna arrays, where the idea is to increase the number of service elements in a predetermined, fixed sized array. Doing this imposes an inversely proportional decrease in the inter-element spacing. The reduced interelement spacings can greatly increase the level of spatial correlation and antenna coupling, as shown in [57, 89-94]. Rather interestingly, the study in [90] demonstrates that in contrast to unconstrained sized arrays which achieve favorable propagation with ever increasing number of antennas, space-constrained arrays do not achieve favorable propagation in the asymptotic regime. Indeed, prior studies (see e.g., [89,93]) have analyzed the impact of space-constrained arrays on MU-MIMO systems in rich scattering environments, with spatial correlation and mutual coupling. However, their impact on propagation channels containing LoS still remains largely unexplored. In this context, Chapter 8 of the thesis analyzes an uplink space-constrained MU-MIMO system with spatially correlated Ricean fading channels, where each terminal has an unequal link gain, unequal levels of LoS and unequal spatial correlation patterns.

This concludes the necessary theoretical background required for the material presented in the thesis. The subsequent chapters present the main contributions of the thesis. 


\section{Chapter 4}

\section{Coordinated RZF Precoding in Multi-Cellular Systems}

Summary. Base station (BS) coordination is viewed as a key ingredient for interference management in cellular networks. This chapter demonstrates the impact of BS coordination on the performance of multi-cellular systems. A general analysis of the average (expected) signal-to-interference-plus-noise-ratio (SINR) of a given user terminal and ergodic sum spectral efficiency for a given cell, with coordinated regularized zero-forcing (RZF) precoding is presented. An application to two-tier small-cellular networks is considered, assuming independent and identically distributed (i.i.d.), as well as semi-correlated Rayleigh fading channels with spatial correlation at the BS. With semi-correlated Rayleigh fading, the generalized analysis methodology caters for the presence of equal and unequal spatial correlation matrices. With i.i.d. Rayleigh fading and when each terminal is assigned an equal correlation matrix, the expressions are averaged over the eigenvalue densities of the instantaneous channel correlation matrices, which follow an uncorrelated and correlated, complex central Wishart distribution. With unequal correlation matrices, the high link signal-to-noiseratio (SNR) convergence of RZF to zero-forcing ( $\mathrm{ZF}$ ) precoding is exploited, where the RZF performance is approximated with ZF. Here, a second order Neumann series expansion is invoked to derive closed-form expressions of the ZF expected per-terminal SNR and ergodic per-cell sum spectral efficiency. The 
derived closed-form results provide numerous valuable insights into the impact of different network parameters, as well as the effect of equal and unequal spatial correlation structures. All derived expressions are robust to changes in system dimensions, high link SNR levels and spatial correlation levels. Numerical results demonstrate the improvement in the above mentioned performance metrics with increasing degrees of BS coordination. It is shown that network-wide coordination provides better inter-cellular interference control, in comparison to cell-wide and macro-only coordination strategies.

\subsection{Introduction}

It is well known that wireless communication systems composed of many uncoordinated transmitters and receivers utilizing the same radio spectrum are interference-limited $[25,26,95]$. This means that a further increase in the level of transmit power does not improve the achievable spectral efficiency, once these powers reach a sufficiently high level. ${ }^{1}$ In contemporary cellular systems, spectrum reuse leads to a large fraction of user terminals having a low signal-tointerference-plus-noise-ratio (SINR) $[10,95] .{ }^{2}$ Similar effects can also be observed in other types of wireless systems, such as in IEEE 802.11 (WiFi), where inefficient contention-based medium access control is employed to protect a particular receiver from interference, by silencing the nearby transmitters. In a by-now vast literature, it has been persuasively argued that this limitation is not fundamental, but rather an artifact of each transmitter-receiver pair communicating autonomously rather than cooperatively (see e.g., [5, 83, 96-104] and the references therein).

Assuming perfect channel state information at the transmitter, if the various transmitters could coordinate, the corresponding downlink interference channel

\footnotetext{
${ }^{1}$ Here the term sufficiently high refers to the transmit power level after the initial gain in the spectral efficiency with increasing the transmit power, assuming a fixed noise power level. Note that this initial gain is often refereed to as the initial offset or the pre-log factor.

${ }^{2}$ Note that here the term cell-edge is referred to as the radio cell-edge and not the geographical (physical) cell-edge, as the presence of random obstacles causing shadow-fading is considered on each propagation path.
} 
can be converted to a broadcast channel, with all the transmitters jointly encoding the signal intended for a given receiver. In the context of a cellular system, this line of thinking would lead us to believe that an arbitrary number of BSs could coordinate to achieve enormous SINR and spectral efficiency gains over uncoordinated systems, with the only limitation being the amount of coordination that can be practically affordable [105]. The problem described above is, and has been of considerable theoretical and practical interest in academia, as well as in the industry (see e.g., $[99,106]$ ). Coordination between multiple BSs has been attempted commercially, and is still ongoing broadly under the banner of coordinated multipoint (CoMP) [106, 107].

Assuming the frequency-division duplex protocol and the availability of cell specific orthogonal reference signaling, the idea is that multiple BSs are connected via low-latency, high bandwidth backhaul links, usually via optical fiber or an external interface 2 (X2) connection. Here, a given user terminal in the system can estimate the downlink channel from the serving and interfering BSs. After quantization of the estimated channels, channel state information is then fed back to the serving BS via a low-rate feedback mechanism. ${ }^{3}$ This allows the desired BS to exchange the channel state information to all interfering BSs via the backhaul interface, where the data is often distributed through a centralized controller, known as the baseband processing unit (BPU) (or a radio network controller (RNC)). ${ }^{4}$ This then allows each BS to form a downlink precoding matrix to terminals in the desired cell of interest, while mitigating or reducing intra-cellular (IUI) ${ }^{5}$ and inter-cellular (ICI) interference, respectively. ${ }^{6}$.

\footnotetext{
${ }^{3}$ Both IEEE $802.11(\mathrm{WiFi})$ and Third Generation Partnership Project (3GPP) long-term evolution advanced (LTE-A) support a so-called "limited feedback" feature, allowing feedback of the quantized subspace information of the channel via a common codebook known to both the transmitter and the receiver [108, 109].

${ }^{4}$ In the case of optical fiber, the exchange of channel state information across multiple BSs is done via a BPU (or a RNC). However, in the case where the X2 interface is used, signaling to and from the BPU (or the RNC) can be bypassed, allowing multiple BSs to directly exchange the data.

${ }^{5}$ Specific to this chapter, multiple types of interference (IUI and ICI) are considered. In the case where only IUI is present, such as in the subsequent chapters, the term "multiuser interference" is used to denote IUI.

${ }^{6}$ At this point, it is important to note that as the focus this chapter is on the mathematical performance analysis of a coordinated multi-cellular systems, an assumption is made in that the above process takes place without any imperfections.
} 
Advances in BS coordination have also been made for small-cellular systems with network densification becoming one of the major themes of future wireless access $[83,99,100]$. In small-cellular systems, different tiers of BSs with varying transmit powers and coverage areas may operate on the same frequency band, in which case ICI rapidly degrades the expected SINR of a given terminal with increasing numbers of overlaid small-cells in the network [83].

On the other hand, with the use of linear transmit and receive signal processing methods, previous studies have analyzed the enhancements to the perterminal SINR and system spectral efficiency with conventional and large antenna arrays $[65,80,89,110-115]$. Collectively, these studies assume independent and identically distributed (i.i.d.) and semi-correlated Rayleigh fading channels. However, in the case of semi-correlated fading, for simplicity, all of the prior work assumes a fixed (common) spatial correlation matrix for each terminal, despite the differences in their physical locations, which contribute to variations in local scattering environment around each terminal. ${ }^{7}$ In addition to the inter-element spacing at the BS array, this significantly influences the resulting performance. In the large system regime, deterministic limits to the terminal SINR and system spectral efficiency with unequal correlation matrices were analyzed in $[41,43,60,116,117]$. A more general limiting analysis with other types of transmit and receive processing techniques, such as regularized ZF (RZF), and minimum-mean-squared-error (MMSE) in the presence of unequal correlation structures is presented in $[60,61]$. However, in the large system regime, the solutions are often presented numerically (see e.g., $[43,60,116]$ ) by iteratively solving a system of fixed point equations, where it is not straightforward to inspect the expressions and evaluate the impact of changes in the system and/or propagation parameters. In contrast to this, explicit analytical expressions that do not require iterative solutions to a set of linked equations is presented in this chapter. The resulting insights are most obvious in the high

\footnotetext{
${ }^{7}$ Keeping in mind the discussion presented in Chapter 3, at this point in the thesis, the author refrains from presenting further discussion regarding the effects of unequal spatial correlation matrices, as a detailed investigation of its impact in a multiuser context is presented further in the thesis, in Chapter 7.
} 
link signal-to-noise-ratio (SNR) regime, where ZF precoding is used to approximate the RZF performance with unequal correlation structures. In general, to derive the expected SINR and ergodic per-cell sum spectral efficiency, averaging over the ensamble of fast-fading is performed, unless otherwise specified in the text.

More specifically, the contributions of the chapter are as follows:

1. Tight analytical expressions are derived to approximate the RZF expected SINR and ergodic spectral efficiency for i.i.d. and semi-correlated Rayleigh fading channels with spatial correlation at the BS array. For equal correlation matrices at each terminal, the expressions are averaged over the arbitrary eigenvalue densities of the instantaneous channel correlation matrices. To the best of the author's knowledge, such an analysis has not been carried out previously and is considered to be extremely cumbersome in $[118,119]$. As a result, $[118,119]$ consider analyzing the expected SINR and ergodic sum spectral efficiency over the isotropic eigenvector matrix for simplicity.

2. With unequal correlation matrices, the high link SNR regime is considered, where RZF precoding converges to ZF precoding. Following the spatial correlation model in $[120,121]$, the RZF performance is approximated with ZF, and a second order Neumann series expansion is invoked to derive closed-form approximations of the expected ZF SNR and ergodic spectral efficiency. In comparison to previous studies, such as $[43,60,116]$, our approximations provide clear insights into the impact of unequal correlation structures, along with other system parameters that influence the achievable expected ZF SNR and ergodic spectral efficiency.

3. For a two-tier small-cellular network, with numerical simulations, the gain in the expected SINR, expected SNR and ergodic sum spectral efficiency is demonstrated with network-wide BS coordination relative to macro-only and cell-wide BS coordination strategies. The impact of network densification on a given terminal and a given cell is explored with uniform, cell-edge 
and cell-centric small-cell placements. The performance of such systems with unequal spatial correlation is superior to that where each terminal is assigned a fixed correlation matrix. Finally, the derived approximations are shown to be robust to variation in the system dimensions, link SNRs and unequal levels of spatial correlation.

The rest of the chapter is structured as follows: Section 4.2 details the multicellular system model and the respective BS coordination strategies. Section 4.3 analyzes the expected per-terminal SINR and ergodic per-cell sum spectral efficiency with i.i.d. and semi-correlated Rayleigh fading channels, where each terminal has an equal correlation matrix. Section 4.4 details a high link SNR approximation to the above mentioned performance metrics with ZF precoding considering unequal correlation matrices for each terminal. Section 4.5 present the numerical performance evaluation of the considered system, and Section 4.6 presents the concluding remarks of the chapter.

\subsection{System Model}

\subsubsection{Downlink Received Signal}

The downlink of a two-tier, multi-cellular, multiuser multiple-input multipleoutput (MU-MIMO) system is considered. ${ }^{8}$ There are $K$ cells in total, where tier one consists of macro and tier two consists of small-cell BSs. BS $k$, located at the origin of cell $k$ in either tier is equipped with an array of $M_{k}$ transmit antennas, simultaneously serving $L_{k}$ non-cooperative single antenna user terminals in the same time-frequency interval. Perfect backhaul links with zero latency and infinite bandwidth are assumed between the $K$ cells. ${ }^{9}$ Furthermore, narrowband transmission and no power control is assumed. The $1 \times M_{k}$ downlink channel vector from BS $k$ to terminal $l$ located in cell $k$ is denoted as $\boldsymbol{g}_{\mathrm{dl}, k, l, k}^{\mathrm{H}}$.

\footnotetext{
${ }^{8}$ As this system model is specific to this chapter, a detailed description of the model is presented in the chapter to maximize clarity and consistency with other chapters.

${ }^{9}$ As the focus is on understanding the best-case performance, the author does not consider specific clusters of coordinated BSs, as typically done in conventional multi-cellular systems (see e.g., [97, 98, 103]).
} 
The ICI channel from BS $j$ to user terminal $l$ in cell $k$ is denoted by $\boldsymbol{g}_{\mathrm{dl}, j, l, k}^{\mathrm{H}}$, where $j \neq k$. Both the desired and interfering channels are assumed to follow correlated Rayleigh fading with $\boldsymbol{g}_{\mathrm{dl}, j, l, k}^{\mathrm{H}}=\boldsymbol{h}_{\mathrm{dl}, j, l, k}^{\mathrm{H}} \boldsymbol{R}_{j, l, k}^{\frac{1}{2}}$, where $\boldsymbol{h}_{\mathrm{dl}, j, l, k}^{\mathrm{H}} \sim \mathcal{C N}\left(0, \boldsymbol{I}_{M_{j}}\right)$ and $\boldsymbol{R}_{j, l, k}$ is the transmit spatial correlation matrix for channel $\boldsymbol{g}_{\mathrm{dl}, j, l, k}^{\mathrm{H}}$. In the case where each terminal has an equal correlation matrix, $\boldsymbol{R}_{j, l, k}=\boldsymbol{R}_{j}, \forall l, k$. While the discussion of the particular structure of $\boldsymbol{R}_{j, l, k}$ is postponed to Section 4.5, the generality of the channel model presented is worth noting. The channel model allows one to consider any type of antenna correlation structure in $\boldsymbol{R}_{j, l, k}$. Although the general case of MU-MIMO in a two-tier small-cellular network is considered, the above model is also of significance to large antenna arrays, where strong antenna correlation may arise due to inadequate inter-element spacing or lack of multi-path diversity [61].

With channel knowledge at BS $k$, the received signal at the $l$-th terminal in the $k$-th cell is given by

$$
\begin{aligned}
r_{\mathrm{dl}, l, k}= & \underbrace{\rho_{\mathrm{dl}, k}^{\frac{1}{2}}\left(\frac{\beta_{k, l, k}}{\eta_{k}}\right)^{\frac{1}{2}} \boldsymbol{g}_{\mathrm{dl}, k, l, k}^{\mathrm{H}} \boldsymbol{w}_{l, k} s_{\mathrm{dl}, l, k}}_{\text {desired signal }}+\underbrace{\rho_{\mathrm{dl}, k}^{\frac{1}{2}}\left(\frac{\beta_{k, l, k}}{\eta_{k}}\right)^{\frac{1}{2}} \sum_{\substack{m=1 \\
m \neq l}}^{L_{k}} \boldsymbol{g}_{\mathrm{dl}, k, l, k}^{\mathrm{H}} \boldsymbol{w}_{m, k} s_{\mathrm{dl}, m, k}}_{\text {intra-cellular interference (IUI) }}+ \\
& \underbrace{\sum_{\substack{j=1 \\
j \neq k}}^{K} \rho_{\mathrm{dl}, j}^{\frac{1}{2}}\left(\frac{\beta_{j, l, k}}{\eta_{j}}\right)^{\frac{1}{2}} \boldsymbol{g}_{\mathrm{dl}, j, l, k}^{\mathrm{H}} \sum_{q=1}^{L_{j}} \boldsymbol{w}_{q, j} s_{\mathrm{dl}, q, j}}_{\text {inter-cellular interference (ICI) }}+\underbrace{n_{\mathrm{dl} l, l, k}}_{\text {noise }},
\end{aligned}
$$

where $\rho_{\mathrm{dl}, k}$ is the average downlink transmit power at BS $k, \boldsymbol{w}_{l, k}$ is the $M_{k} \times 1$ un-normalized precoding vector for the $l$-th terminal in cell $k$ and $\eta_{k}$ is the precoder normalization parameter, (discussed later in the text) for the $k$-th cell. $s_{\mathrm{dl}, l, k}$ is the transmitted data symbol from BS $k$ to terminal $l$ with unit mean power and $n_{\mathrm{dl}, l, k}$ denotes white Gaussian noise at the $l$-terminal where $n_{\mathrm{dl}, l, k} \sim \mathcal{C N}\left(0, \sigma_{\mathrm{dl}, l, k}^{2}\right)$. Note that for simplicity, $\sigma_{\mathrm{dl} l, l, k}^{2}=\sigma_{\mathrm{dl}}^{2}, \forall l, k$, respectively. Hence, the link SNR is defined as the ratio of $\rho_{\mathrm{dl}, k}$ to $\sigma_{\mathrm{dl}}^{2}$ for an arbitrary link from BS $k$ to any given terminal in the system. The desired and interfering link gains at the $l$-th terminal in the $k$-th cell from the desired and interfering 
links are denoted by $\beta_{k, l, k}$ and $\beta_{j, l, k}$, respectively. The link gains from BS $j$ to terminal $l$ in cell $k$ is modeled as [15]

$$
\beta_{j, l, k}=v\left(\frac{d_{0}}{d_{j, l, k}}\right)^{\alpha_{j}} \zeta_{j, l, k}
$$

Here, $v$ is a unit-less constant for geometric attenuation at the reference distance $d_{0}$, assuming far-field, omni-directional transmit antennas with an ideal radiation pattern and no mechanical downtilt. Furthermore, $d_{j, l, k}$ is the distance from BS $j$ to terminal $l$ in cell $k$ and $\alpha_{j}$ is the attenuation exponent dependent on the transmitting BS and the propagation scenario. The random effects of shadowfading are modeled with a log-normal density, such that $\zeta_{j, l, k}=10^{\left(S \sigma_{\mathrm{sf}} / 10\right)}$, where $S \sim \mathcal{N}(0,1)$ and $\sigma_{\text {sf }}$ is the shadow-fading standard deviation.

Fig. 4.1 depicts a typical two-tier cellular network composed of macrocells with multiple overlaid small-cells. Here the effects of a downlink broadcast to terminal 1 in macrocell 7's coverage area is demonstrated, as it causes IUI to terminal 2 in macrocell 7 , as well as experiencing ICI from the nearby smallcells (small-cells 1 and 4). Note that terminal 2 of macrocell 7 also experiences ICI from small-cells 1 and 2. In order to avoid the possibility of very large levels of interference powers (from this model), a physical exclusion radius is designed around the macro BS in which no small-cell BSs are placed. Each BS has an optical fiber backhaul link to the BPU. While Fig. 4.1 is only used for illustration purposes, more insightful scenarios (with variation in the small-cell placement) are considered in Section 4.5 to evaluate the expected per-terminal and cell-wide performance.

\subsubsection{RZF Precoding}

RZF precoding is considered in the design of the downlink precoding vectors to each terminal [118]. ${ }^{10}$ Firstly, the focus is on the case where each serving array performs RZF to the user terminals in its own coverage area, and later

\footnotetext{
${ }^{10}$ As a multi-cellular system model is employed specifically for this chapter, the signal model, as well as the RZF precoding vectors are restated for clarity and ease of reference.
} 


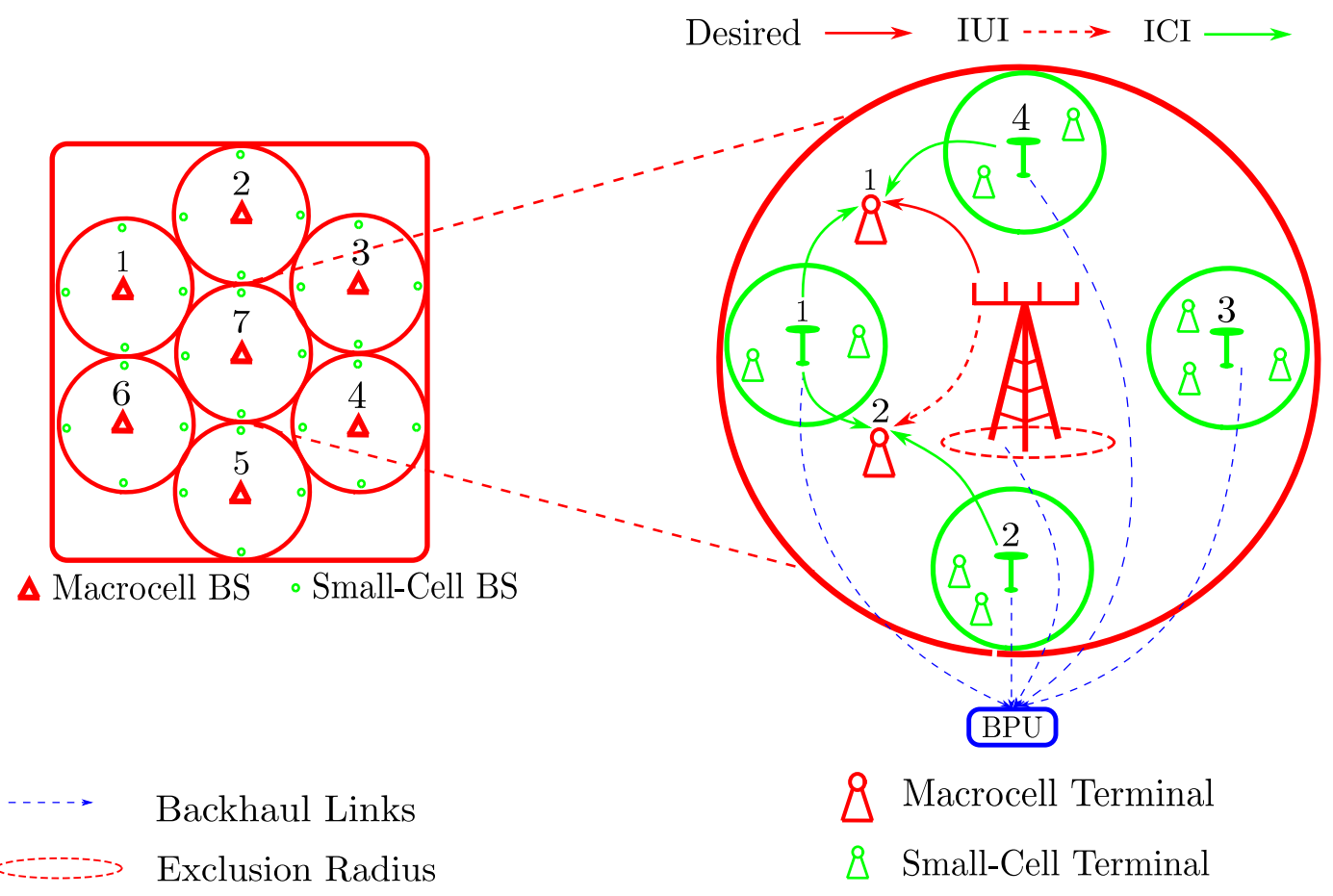

Figure 4.1: Two-tier multi-cellular network with uniformly distributed smallcells.

this is extended to other BS coordination strategies. The un-normalized RZF precoding vector for the $l$-th terminal in cell $k$ is the $l$-th column of the $M_{k} \times L_{k}$ matrix, $\boldsymbol{W}_{k}$, such that

$$
\boldsymbol{W}_{k}=\boldsymbol{G}_{\mathrm{dl}, k}\left(\boldsymbol{G}_{\mathrm{dl}, k}^{\mathrm{H}} \boldsymbol{G}_{\mathrm{dl}, k}+\xi_{k} \boldsymbol{I}_{L_{k}}\right)^{-1}
$$

where $\boldsymbol{G}_{\mathrm{dl}, k}=\left[\boldsymbol{g}_{\mathrm{dl}, k, 1, k}, \boldsymbol{g}_{\mathrm{dl}, k, 2, k}, \ldots, \boldsymbol{g}_{\mathrm{dl}, k, L_{k}, k}\right]$ is the $M_{k} \times L_{k}$ composite channel matrix containing downlink channel vectors of all $L_{k}$ terminals in cell $k$ (resulting in $\boldsymbol{G}_{\mathrm{dl}, k}^{\mathrm{H}}$ which of size $L_{k} \times M_{k}$ ). Following [43], the precoding matrix in (4.3) is normalized via $\eta_{k}=\left\|\boldsymbol{W}_{k}\right\|^{2} / L_{k}$ ensuring $\mathbb{E}\left[\left\|\boldsymbol{w}_{l, k}\right\|^{2}\right]=1$. The constant $\xi_{k}>0$ denotes the regularization parameter specific to BS $k$. In line with [122]

$$
\xi_{k}=\frac{1}{K L} \sum_{k=1}^{K} \sum_{\substack{l=1 \\ l \neq k}}^{L_{k}} \frac{1}{\beta_{k, l, k}}
$$

where $K$ and $L$ are the total number of cells and terminals within the system, respectively. While selecting $\xi_{k}$ to maximize the SINR has been considered in 
[118] for a single-cell system, maximization of SINR in the case of multiple cells leads to a coupled optimization problem [118]. Optimization of $\xi_{k}$ is treated as a separate issue and is beyond the scope of the chapter and thesis. It is worth mentioning that the computation of $\xi_{k}$ in (4.4) requires BS $k$ to know all large-scale fading (typically also known as slow-fading) co-efficients via the backhaul infrastructure. This assumption is reasonable, since designing a RZF precoding matrix at BS $k$ requires knowledge of the instantaneous channel state information. Since the large-scale fading co-efficients vary much more slowly than the fast-fading, they can be acquired with much less effort than the instantaneous channel. ${ }^{11}$ In the sequel, the proposed BS coordination strategies are presented for the two-tier small-cellular network, along with the corresponding formulation of the composite channel matrix used for RZF processing.

\subsubsection{Base Station Coordination Strategies}

\section{Cell-Wide Coordination}

In this strategy, the BSs in both tiers perform single-cell RZF precoding. That is, each BS coordinates the desired and IUI locally at the serving antenna array. However, in this strategy, ICI is still present from other BSs, as the RZF precoder does not consider any ICI channels in its design. The composite channel matrix to compute the RZF precoders for $L_{k}$ terminals in cell $k$ is given by

$$
\boldsymbol{G}_{\mathrm{dl}, k}=\left[\boldsymbol{g}_{\mathrm{dl}, k, 1, k}, \boldsymbol{g}_{\mathrm{dl}, k, 2, k}, \ldots, \boldsymbol{g}_{\mathrm{dl}, k, L_{k}, k}\right]
$$

One can think of this strategy as the baseline case, which will be useful for comparison purposes with the best-case performance described in the networkwide coordination strategy below.

\footnotetext{
${ }^{11}$ It is typically reported that large-scale fading co-efficients vary approximately 40 times less frequently than the instantaneous fast-fading channel. The interested reader is referred to [37] for instance, for further discussion on this matter.
} 


\section{Network-Wide Coordination}

In this strategy, the serving BS array applies RZF precoding not only to the channels of its own terminals, but also considers ICI to other terminals in the system. The serving and interfering links can be determined using cell-specific pilot signaling (assuming perfect channel estimation in the absence of pilot contamination and the availability of a sufficient number of orthogonal reference signals). All interfering channel state information from other cells is delivered to the serving cell BS via the backhaul interface. The composite channel matrix, $\boldsymbol{G}_{\mathrm{d} l, k}$, for cell $k$ with network-wide coordinated RZF processing can now be defined as

$$
\boldsymbol{G}_{\mathrm{dl}, k}=\left[\boldsymbol{Z}_{\mathrm{dl}, 1}, \boldsymbol{Z}_{\mathrm{dl}, 2}, \ldots, \boldsymbol{Z}_{\mathrm{dl}, j}, \ldots, \boldsymbol{Z}_{\mathrm{dl}, K}\right]
$$

where $\boldsymbol{Z}_{\mathrm{dl}, j}=\left[\boldsymbol{g}_{\mathrm{dl}, j, 1, k}, \ldots, \boldsymbol{g}_{\mathrm{dl}, j, L_{k}, k}\right]$. With this composite channel matrix, (4.3) can still be used, however, the precoder $\boldsymbol{W}_{k}$ only contains the $L_{k}$ columns of (4.3) corresponding to the terminals in cell $k$ (columns $\sum_{i=1}^{k-1} L_{i}+1$ to $\sum_{i=1}^{k} L_{i}$ ). Similarly, $\eta_{k}$ is the Frobenius norm of $\boldsymbol{W}_{k}$ with these $L_{k}$ columns only. Since network-wide coordination requires knowledge of all instantaneous channel vectors at $\mathrm{BS} k$, it introduces significant backhaul overheads. While this may not be practical, such a strategy allows us to evaluate the best case (upper bound) performance of the expected per-terminal SINR and ergodic per-cell spectral efficiency in such interference limited scenarios.

\section{Macro-Only Coordination}

In this strategy, it is assumed that the macrocell BSs have knowledge of the ICI channels to terminals located in tier-two (small) cells. The macrocells then utilize this out-of-cell channel state information to coordinate the downlink transmission to its own, as well as to terminals in tier-two cells. This strategy is useful since it attempts to suppress the most dominant sources of interference, the macro BSs, in the two-tier network. ${ }^{12}$ The composite channel used to obtain

\footnotetext{
${ }^{12}$ It is well known that the transmit powers of small-cellular BSs are scaled down to serve a smaller coverage area, leading to comparatively lower levels of ICI than the high powered macro BS.
} 
the RZF precoding matrix for BS $k$ is equivalent to (4.6) and (4.5), if BS $k$ is a macro and microcell, respectively.

The following section presents the methodology to analyze expected RZF SINR and ergodic per-cell sum spectral efficiency under i.i.d. and semi-correlated Rayleigh fading channels, with the above mentioned coordination strategies. However, before presenting the analysis methodology, the integrals and special functions that are used throughout the remainder of the chapter are defined.

\subsection{Analysis Methodology}

\subsubsection{Integrals and Special Functions}

Throughout the chapter, the following integrals and special functions are often used. Let

$$
J_{a, b, c}(\xi)=\int_{0}^{\infty} \frac{\lambda^{a} e^{-\lambda / c}}{(\lambda+\xi)^{b}} d \lambda, \quad \text { where } a, b \text { and } c \geq 1 .
$$

The integral in (4.7) can be solved by substituting $\lambda=\omega-\xi$ to obtain

$$
J_{a, b, c}(\xi)=\int_{\xi}^{\infty} \frac{(\omega-\xi)^{a} e^{-(\omega-\xi) / c}}{\omega^{b}} d \omega=\sum_{f=0}^{a}\left(\begin{array}{l}
a \\
f
\end{array}\right)(-\xi)^{a-f} e^{\xi / c} \underbrace{\int_{\xi}^{\infty} \omega^{f-b} e^{-\omega} d \omega}_{J^{(b)}} .
$$

For the purpose of this chapter, two special cases of $J^{(b)}(\xi)$ are of interest, when $b=1$ and $b=2$. These are given by

$$
\begin{aligned}
& J^{(1)}(\xi)=\int_{\xi}^{\infty} \omega^{f-1} e^{-\omega} d \omega=\left\{\begin{array}{ll}
\operatorname{Ei}(1, \xi) & \text { if } f=0 \\
\Gamma(f, \xi) & \text { if } f \geq 1,
\end{array}\right. \text { and } \\
& J^{(2)}(\xi)=\int_{\xi}^{\infty} \omega^{f-2} e^{-\omega} d \omega= \begin{cases}-\operatorname{Ei}(1, \xi)+\frac{e^{-\xi}}{\xi^{2}} & \text { if } f=0 \\
\operatorname{Ei}(1, \xi) & \text { if } f=1 \\
\Gamma(f-1, \xi) & \text { if } f \geq 2,\end{cases}
\end{aligned}
$$


where $\operatorname{Ei}(\cdot, \cdot)$ is the generalized exponential integral and $\Gamma(\cdot, \cdot)$ is the incomplete gamma function [85].

\subsubsection{Expected SINR and Ergodic Sum Spectral Effi- ciency Analysis}

From (4.1), the SINR at the $l$-th terminal in cell $k$ being served by BS $k$ is a random quantity which depends on the random terminal location, log-normally distributed shadow-fading and local multi-path fading can be written as

$$
\mathrm{SINR}_{\mathrm{dl}, l, k}=\frac{\rho_{\mathrm{dl}, k} \frac{\beta_{k, l, k}}{\eta_{k}}\left|\boldsymbol{g}_{\mathrm{dl}, k, l, k}^{\mathrm{H}} \boldsymbol{w}_{l, k}\right|^{2}}{\sigma_{\mathrm{dl}, l, k}^{2}+\rho_{\mathrm{dl}, k} \frac{\beta_{k, l, k}}{\eta_{k}} \sum_{\substack{m=1 \\ m \neq l}}^{L_{k}}\left|\boldsymbol{g}_{\mathrm{dl}, k, l, k}^{\mathrm{H}} \boldsymbol{w}_{m, k}\right|^{2}+\sum_{\substack{j=1 \\ j \neq k}}^{K} \rho_{\mathrm{dl}, j} \frac{\beta_{j, l, k}}{\eta_{j}} \sum_{q=1}^{L_{j}}\left|\boldsymbol{g}_{\mathrm{dl}, j, l, k}^{\mathrm{H}} \boldsymbol{w}_{q, j}\right|^{2}} .
$$

From (4.11), the expected per-terminal SINR can be obtained by computing $\mathbb{E}\left[\mathrm{SINR}_{\mathrm{dl}, l, k}\right]$. As motivated in Chapter 3, Section 3.4.3, exact evaluation of $\mathbb{E}\left[\mathrm{SINR}_{\mathrm{dl} l, l, k}\right]$ is extremely cumbersome, if not intractable. Hence, the commonly used first-order delta method expansion is employed, as demonstrated in the analysis methodology of $[68,80,84,86,87,93]$. Following this, the expected SINR can be approximated by

$$
\mathbb{E}\left[\mathrm{SINR}_{\mathrm{d} l, l, k}\right] \approx \frac{\rho_{\mathrm{dl}, k} \frac{\beta_{k, l, k}}{\tilde{\eta}_{k}} \mathbb{E}\left[\left|\boldsymbol{g}_{\mathrm{dl}, k, l, k}^{\mathrm{H}} \boldsymbol{w}_{l, k}\right|^{2}\right]}{\sigma_{\mathrm{dl} l, l, k}^{2}+\rho_{\mathrm{dl}, k} \frac{\beta_{k, l, k}}{\tilde{\eta}_{k}} \sum_{\substack{m=1 \\ m \neq l}}^{L_{k}} \mathbb{E}\left[\left|\boldsymbol{g}_{\mathrm{dl}, k, l, k}^{\mathrm{H}} \boldsymbol{w}_{m, k}\right|^{2}\right]+\sum_{\substack{j=1 \\ j \neq k}}^{K} \rho_{\mathrm{dl}, j} \frac{\beta_{j, l, k}}{\tilde{\eta}_{j}} \sum_{q=1}^{L_{j}} \mathbb{E}\left[\left|\boldsymbol{g}_{\mathrm{dl}, j, l, k}^{\mathrm{H}} \boldsymbol{w}_{q, j}\right|^{2}\right]},
$$

where the quantities $\tilde{\eta}_{k}=\mathbb{E}\left[\eta_{k}\right]$ and $\tilde{\eta}_{j}=\mathbb{E}\left[\eta_{j}\right]$, respectively. The resulting ergodic spectral efficiency for terminal $l$ in cell $k$ (in bits/seconds/Hz) is given by $\mathbb{E}\left[\mathrm{R}_{\mathrm{dl}, l, k}\right]=\mathbb{E}\left[\log _{2}\left(1+\mathrm{SINR}_{\mathrm{dl}, l, k}\right)\right]$. As such, the ergodic sum spectral efficiency for the $L_{k}$ terminals in cell $k$ can be approximated as

$$
\mathbb{E}\left[\mathrm{R}_{\mathrm{sum}, \mathrm{dl}, k}\right]=\mathbb{E}\left[\sum_{l=1}^{L_{k}} \log _{2}\left(1+\operatorname{SINR}_{\mathrm{dl}, l, k}\right)\right] \approx \sum_{l=1}^{L_{k}} \log _{2}\left(1+\mathbb{E}\left[\operatorname{SINR}_{\mathrm{dl}, l, k}\right]\right)
$$

Remark 4.3.1. Note that (4.13) leads to an approximation rather than 
an upper bound via Jensen's inequality, as the value of $\mathbb{E}\left[\operatorname{SINR}_{\mathrm{dl}, l, k}\right]$ is itself an approximation [123]. Furthermore, the summation over all $L_{k}$ terminals in cell $k$ takes care of the fact that $\mathbb{E}\left[\mathrm{SINR}_{\mathrm{dl}, l, k}\right]$ could be different depending on $l$ and $k$, due to the relative differences in the physical location of the terminal, causing further differences in the level of geometric attenuation and log-normal shadow-fading.

In the following subsections, the expected values in the numerator and denominator of (4.12) are derived separately for i.i.d. and semi-correlated Rayleigh fading channels with equal correlation matrices for each terminal.

\section{Expected Signal Power}

The expected signal power in (4.12) is given by

$$
\delta_{l, k}=\rho_{\mathrm{dl}, k} \frac{\beta_{k, l, k}}{\tilde{\eta}_{k}} \mathbb{E}\left[\left|\boldsymbol{g}_{\mathrm{dl}, k, l, k}^{\mathrm{H}} \boldsymbol{w}_{l, k}\right|^{2}\right]
$$

Via an eigenvalue decomposition, $\boldsymbol{G}_{\mathrm{dl}, k}^{\mathrm{H}} \boldsymbol{G}_{\mathrm{dl}, k}=\boldsymbol{U} \boldsymbol{\Lambda} \boldsymbol{U}^{\mathrm{H}}$. Then, the expected value in (4.14) over the isotropic distribution of $\boldsymbol{U}$ can be written as [118]

$$
\varrho_{l, k}=\mathbb{E}\left[\left|\boldsymbol{g}_{\mathrm{dl}, k, l, k}^{\mathrm{H}} \boldsymbol{w}_{l, k}\right|^{2}\right]=\mathbb{E}\left[\left(\sum_{l=1}^{m} \frac{\lambda_{l}}{\lambda_{l}+\xi_{k}}\left|u_{k, l}\right|^{2}\right)^{2}\right]
$$

where $m$ is the minimum of the transmit and receive dimensions. For cellwide coordination, $m=\min \left(L_{k}, M_{k}\right)$, while for network-wide coordination, $m=\min \left(\sum_{k=1}^{K} L_{k}, M_{k}\right)$. Equivalently, it is to be noted that $n=\max \left(L_{k}, M_{k}\right)$ for cell-wide coordination and $n=\max \left(\sum_{k=1}^{K} L_{k}, M_{k}\right)$ for network-wide coordination. ${ }^{13}$ Moreover, $\lambda_{l}$ is the $l$-th eigenvalue corresponding to the $l$-th diagonal entry in $\boldsymbol{\Lambda}$ and $u_{k, l}$ denotes the $(k, l)$-th entry of $\boldsymbol{U}$. The expression in (4.15)

\footnotetext{
${ }^{13}$ Note that the case of macro-only coordination strategy is not specifically presented, since this case is captured in either the cell-wide or network-wide coordination, depending on the BS type (macro or small-cell) of interest.
} 
can be further averaged over the entries of $\boldsymbol{U}$ and can be written as [118]

$$
\varrho_{l, k}=\frac{1}{m(m+1)}\left\{\mathbb{E}_{\lambda}\left[\left(\sum_{l=1}^{m} \frac{\lambda_{l}}{\lambda_{l}+\xi_{k}}\right)^{2}\right]+\mathbb{E}_{\lambda}\left[\sum_{l=1}^{m}\left(\frac{\lambda_{l}}{\lambda_{l}+\xi_{k}}\right)^{2}\right]\right\}
$$

where $\mathbb{E}_{\lambda}[\cdot]$ denotes the expectation over the eigenvalues of the complex central Wishart matrices. From here, $\tilde{\eta}_{k}$ can also be inferred as

$$
\tilde{\eta}_{k}=\tau \mathbb{E}\left[\left\|\boldsymbol{W}_{k}\right\|_{\mathrm{F}}^{2}\right]=\tau \mathbb{E}_{\lambda}\left[\sum_{l=1}^{m} \frac{\lambda_{l}}{\left(\lambda_{l}+\xi_{k}\right)^{2}}\right]
$$

where $\tau=\frac{1}{L_{k}}$ for cell-wide and $\frac{1}{\sum_{k=1}^{K} L_{k}}$ for network-wide coordination (see footnote 11), as every column of $\boldsymbol{W}_{k}$ is identically distributed.

Remark 4.3.2. The above method to compute the expected per-terminal signal power can be applied to any eigenvalue distribution of the channel matrix. ${ }^{14}$ The analysis in this chapter only uses the fact that the eigenvector matrix $\boldsymbol{U}$ has the so-called isotropic structure, whose defining characteristic is that pre or post multiplying $\boldsymbol{U}$ by any unitary matrix does not affect its distribution (see e.g., [71, 118]). Physically, this means that the channel is not affected by arbitrary rotations, and that paths between the service antennas and terminals are statistically equivalent. It is this feature that allows one to examine the signal power (and interference power for that matter) and claim that the analysis applies equally to the remaining terminals in the system. The analysis methodology, therefore, applies to other channel distributions with this rotational-invariance property, other than the complex Gaussian [118].

The expressions in (4.16) and (4.17) can be further averaged over the density of the eigenvalues as shown in the following Theorems and Lemmas for uncorrelated and semi-correlated Rayleigh fading channels with equal correlation matrices.

Theorem 4.3.1. When $\boldsymbol{R}_{k}=\boldsymbol{I}_{m}$, the expected value of $\sum_{i=1}^{m} \frac{\lambda_{i}^{\mu}}{\left(\lambda_{i}+\xi_{k}\right)^{2}}$, over

\footnotetext{
${ }^{14}$ Even though the remark is made at this point in the chapter, the analysis methodology also applies to the the computation of the interference powers.
} 
the eigenvalues of $\boldsymbol{G}_{\mathrm{dl}, k}^{\mathrm{H}} \boldsymbol{G}_{\mathrm{dl}, k}$ is given by

$$
S_{k}^{\mu}=\sum_{i=1}^{m} \frac{(i-1) !}{(i-1+n-m) !} \sum_{z=0}^{i-1} \sum_{\substack{l=0 \\ l \neq z}}^{i-1}(-1)^{z+l}\left(\kappa_{z}\right)\left(\kappa_{l}\right) \frac{1}{z ! l !} J_{\mu+n-m+z-l, 2,1}\left(\xi_{k}\right),
$$

where $\kappa_{z}=\left(\begin{array}{c}i-1+n-m \\ i-1-z\end{array}\right), \kappa_{l}=\left(\begin{array}{c}i-1+n-m \\ i-1-l\end{array}\right)$ and $J_{\mu+n-m+z-l, 2,1}\left(\xi_{k}\right)$ is as defined in (4.8) with $\mu>0$.

Proof of Theorem 4.3.1. Recognizing the fact that

$$
S_{k}^{\mu}=\mathbb{E}_{\lambda}\left[\sum_{i=1}^{m} \frac{\lambda_{i}^{\mu}}{\left(\lambda_{i}+\xi_{k}\right)^{2}}\right]=m\left[\int_{0}^{\infty} \frac{\lambda_{\text {arb }}^{\mu}}{\left(\lambda_{\text {arb }}+\xi_{k}\right)^{2}} f_{0}\left(\lambda_{\text {arb }}\right) d \lambda_{\text {arb }}\right]
$$

it can be noted that $\mathbb{E}_{\lambda}[\cdot]$ denotes the expectation over the eigenvalues and $f_{0}\left(\lambda_{\mathrm{arb}}\right)$ is the density of an arbitrary eigenvalue, $\lambda_{\mathrm{arb}}$, from $\left\{\lambda_{1}, \ldots, \lambda_{m}\right\}$. To evaluate (4.19) for an uncorrelated central Wishart matrix, the probability density from (2.22) in Chapter 2 is substituted into (4.19), allowing one to state

$$
S_{k}^{\mu}=m\left\{\int_{0}^{\infty} \frac{\lambda_{\mathrm{arb}}^{\mu}}{\left(\lambda_{\mathrm{arb}}+\xi_{k}\right)^{2}} \frac{1}{m} \sum_{i=1}^{m} \frac{(i-1) ! \lambda_{\mathrm{arb}}^{n-m} e^{-\lambda}}{(i-1+n-m) !}\left[\sum_{z=0}^{i-1}(-1)^{z}\left(\kappa_{z}\right) \frac{\lambda_{\mathrm{arb}}^{z}}{z !}\right]^{2} d \lambda_{\mathrm{arb}}\right\} .
$$

After extracting the relevant constants from (4.20), it can be written as

$$
S_{k}^{\mu}=\sum_{i=1}^{m} \frac{(i-1) !}{(i-1+n-m) !} \sum_{z=0}^{i-1} \sum_{\substack{l=0 \\ l \neq z}}^{i-1}(-1)^{z+l}\left(\kappa_{z}\right)^{2} \frac{1}{z ! l !}\left[\int_{0}^{\infty} \frac{\lambda_{\mathrm{arb}}^{\mu+n-m+z-l} e^{-\lambda_{\mathrm{arb}}}}{\left(\lambda_{\mathrm{arb}}+\xi_{k}\right)^{2}} d \lambda_{\mathrm{arb}}\right]
$$

The integral in (4.21) has the form of $J_{a, b, c}\left(\xi_{k}\right)$ defined in (4.8), where $a=$ $\mu+n-m+z-l, b=2$ and $c=1$. Following the solution provided in (4.10) yields the desired expression in (4.19), concluding the proof.

Theorem 4.3.2. For any $M_{k}, L_{k}$ where $M_{k} \leq L_{k}$ or $M_{k}>L_{k}$, where $\theta_{1}, \ldots, \theta_{n}$ are the eigenvalues of $\boldsymbol{R}_{k} \neq \boldsymbol{I}_{n}$, the expected value of $\sum_{i=1}^{m} \frac{\lambda_{i}^{\bar{\mu}}}{\left(\lambda_{i}+\xi_{k}\right)^{2}}$, 
over the eigenvalues of $\boldsymbol{G}_{\mathrm{dl}, k}^{\mathrm{H}} \boldsymbol{G}_{\mathrm{dl}, k}$ is given by

$$
\bar{S}_{k}^{\bar{\mu}}=\frac{1}{\prod_{i<j}^{n}\left(\theta_{j}-\theta_{i}\right)} \sum_{l=1}^{n} \sum_{k=n-m+1}^{n} \frac{\theta_{l}^{n-m-1} \mathcal{D}_{l, k}}{\Gamma(m-n+k)} J_{m+k-n-1+\bar{\mu}, 2, \theta_{l}}\left(\xi_{k}\right)
$$

where $\mathcal{D}_{l, k}$ is the $(l, k)$-th co-factor of a $n \times n$ matrix whose $(l, k)$-th entry is given by $\theta_{l}^{k-1}, \Gamma(m-n+k)=(m-n+k-1)$ ! is the Gamma function and $J_{m+k-n-1+\bar{\mu}, 2, \theta_{l}}\left(\xi_{k}\right)$ is as defined in (4.8), respectively.

Proof of Theorem 4.3.2. Substituting the arbitrary eigenvalue density, i.e., the density of $\lambda_{\mathrm{arb}, \mathrm{sc}}$, defined in Chapter 2, (2.27), into (4.19) results in

$$
\begin{aligned}
\bar{S}_{k}^{\bar{\mu}}= & m\left[\int_{0}^{\infty} \frac{\lambda_{\mathrm{arb}, \mathrm{sc}}^{\bar{\mu}}}{\left(\lambda_{\mathrm{arb}, \mathrm{sc}}+\xi_{k}\right)^{2}} \frac{1}{m \prod_{i<j}^{n}\left(\theta_{j}-\theta_{i}\right)} \sum_{l=1}^{n} \sum_{k=n-m+1}^{n} \lambda_{\mathrm{arb}, \mathrm{sc}}^{m+k-n-1}\right. \\
& \left.\times e^{-\lambda_{\mathrm{arb}, \mathrm{sc}} / \theta_{l}} \theta_{l}^{n-m-1} \frac{1}{\Gamma(m-n+k)} \mathcal{D}_{l, k} d \lambda_{\mathrm{arb}, \mathrm{sc}}\right]
\end{aligned}
$$

Some mathematical simplification allows (4.23) to be expressed as

$$
\begin{aligned}
\bar{S}_{k}^{\bar{\mu}}=\frac{1}{\prod_{i<j}^{n}\left(\theta_{j}-\theta_{i}\right)} & \sum_{l=1}^{n} \sum_{k=n-m+1}^{n} \frac{\theta_{l}^{n-m-1} \mathcal{D}_{l, k}}{\Gamma(m-n+k)} \\
& \times\left[\int_{0}^{\infty} \frac{\lambda_{\mathrm{arb}, \mathrm{sc}}^{m+k-n-1+\bar{\mu}} e^{-\lambda_{\mathrm{sc}} / \theta_{l}}}{\left(\lambda_{\mathrm{arb}, \mathrm{sc}}+\xi_{k}\right)^{2}} d \lambda_{\mathrm{arb}, \mathrm{sc}}\right],
\end{aligned}
$$

where the integral in (4.24) is of the form of $J_{a, b, c}(\xi)$ in (4.8), where $a=m+k-$ $n-1+\bar{\mu}, b=2$ and $c=\theta_{l}$, where $\theta_{l}$ is the $l$-th eigenvalue of $\boldsymbol{R}_{k}$. Substituting the result of (4.10) into the required integral yields the desired expression in (4.22).

Lemma 4.3.1. When $\boldsymbol{R}_{k}=\boldsymbol{I}_{m}$, the expected value of $\left(\sum_{i=1}^{m} \frac{\lambda_{i}}{\lambda_{i}+\xi_{k}}\right)^{2}$ is 
given by

$$
\begin{aligned}
Q_{k}= & S_{k}^{2}+\sum_{i=1}^{m} \sum_{\substack{j=1 \\
j \neq i}}^{m} \Phi\left[\left(\sum_{f=0}^{i-1} \sum_{\substack{z=0 \\
z \neq f}}^{i-1}(-1)^{f+z}\left(\kappa_{f}\right)\left(\kappa_{z}\right) \frac{1}{f ! z !} J_{n-m+1-f+z, 1,1}\left(\xi_{k}\right)\right)^{2}\right. \\
& \left.\times\left(\sum_{f=0}^{i-1} \sum_{\substack{z=0 \\
z \neq f}}^{i-1}(-1)^{f+z}\left(\kappa_{f}\right)\left(\kappa_{f}\right) \frac{1}{f ! z !} J_{n-m+1+f+z, 1,1}\left(\xi_{k}\right)\right)^{2}\right]
\end{aligned}
$$

where $\Phi=((i-1) !(j-1) !) /((i-1+n-m) !(j-1+n-m) !), \kappa_{f}=\left(\begin{array}{c}n-m+i-1 \\ i-1-f\end{array}\right)$, $\kappa_{z}=\left(\begin{array}{c}n-m+i-1 \\ i-1-z\end{array}\right)$ and $J_{n-m+1+f+z, 1,1}\left(\xi_{k}\right)$ is as defined in (4.8), respectively.

Proof of Lemma 4.3.1. Via first principles, one can recognize that

$\mathbb{E}\left[\left(\sum_{i=1}^{m} \frac{\lambda_{i}}{\lambda_{i}+\xi_{k}}\right)^{2}\right]$ can be written as

$$
\begin{aligned}
& Q_{k}=\mathbb{E}_{\lambda} {\left[\left(\sum_{l=1}^{m} \frac{\lambda_{l}}{\lambda_{l}+\xi_{k}}\right)^{2}\right]=\mathbb{E}_{\lambda}\left[\sum_{l=1}^{m}\left(\frac{\lambda_{l}}{\lambda_{l}+\xi_{k}}\right)^{2}\right] } \\
&+\mathbb{E}_{\lambda}\left[\sum_{\substack{a=1 \\
a=1}}^{m}\left(\frac{\lambda_{a}}{\lambda_{a}+\xi_{k}}\right)\left(\frac{\lambda_{b}}{\lambda_{b}+\xi_{k}}\right)\right] \\
&=m\left\{\mathbb{E}_{\lambda}\left[\frac{1}{m} \sum_{l=1}^{m}\left(\frac{\lambda_{l}}{\lambda_{l}+\xi_{k}}\right)^{2}\right]\right\}+m(m-1) \\
& \quad \times\left\{\mathbb{E}_{\lambda}\left[\frac{1}{m(m-1)} \sum_{\substack{a=1 \\
a=1}}^{m} \sum_{\substack{b=1 \\
b \neq 1}}^{m}\left(\frac{\lambda_{a}}{\lambda_{a}+\xi_{k}}\right)\left(\frac{\lambda_{b}}{\lambda_{b}+\xi_{k}}\right)\right]\right\},
\end{aligned}
$$

where $\mathbb{E}_{\lambda}[\cdot]$ denotes expectation over the eigenvalues and $\lambda_{a}, \lambda_{b}$ denote an arbitrary pair of eigenvalues. Via the result derived in Theorem 4.3.1, the first term of (4.26) can be evaluated. By denoting the joint density of two arbitrary eigenvalues $\left(\lambda_{a}, \lambda_{b}\right)$ as $f_{\mathrm{o}}\left(\lambda_{a}, \lambda_{b}\right)$, one can write (4.26) as

$$
Q_{k}=S_{k}^{2}+m(m-1)\left[\int_{0}^{\infty} \int_{0}^{\infty}\left(\frac{\lambda_{a}}{\lambda_{a}+\xi_{k}}\right)\left(\frac{\lambda_{b}}{\lambda_{b}+\xi_{k}}\right) f_{0}\left(\lambda_{a}, \lambda_{b}\right) d \lambda_{b} d \lambda_{a}\right]
$$

Using the joint density, $f_{\mathrm{o}}\left(\lambda_{a}, \lambda_{b}\right)$, presented in (2.24) of Chapter 2 allows one 
to write $(4.27)$ as

$$
\begin{aligned}
Q_{k} & =S_{k}^{2}+m(m-1)\left\{\int_{0}^{\infty} \int_{0}^{\infty}\left(\frac{\lambda_{a}}{\lambda_{a}+\xi_{k}}\right)\left(\frac{\lambda_{b}}{\lambda_{b}+\xi_{k}}\right) \frac{1}{m(m-1)} \sum_{i=1}^{m} \sum_{\substack{j=1 \\
j \neq i}}^{m}\left(\lambda_{a} \lambda_{b}\right)^{n-m}\right. \\
& \times e^{-\left(\lambda_{a}+\lambda_{b}\right)} \Phi\left[\kappa_{i-1}^{(n-m)}\left(\lambda_{a}\right)^{2} \kappa_{j-1}^{(n-m)}\left(\lambda_{b}\right)^{2}-\kappa_{i-1}^{(n-m)}\left(\lambda_{a}\right) \kappa_{j-1}^{(n-m)}\left(\lambda_{a}\right) \kappa_{i-1}^{(n-m)}\left(\lambda_{b}\right)\right. \\
& \left.\left.\times \kappa_{j-1}^{(n-m)}\left(\lambda_{b}\right)\right] d \lambda_{b} d \lambda_{a}\right\} .
\end{aligned}
$$

After some simplifications, (4.28) can be written as

$$
\begin{aligned}
Q_{k}= & S_{k}^{2}+\sum_{i=1}^{m} \sum_{\substack{j=1 \\
j \neq i}}^{m} \Phi\left\{\int _ { 0 } ^ { \infty } \int _ { 0 } ^ { \infty } \frac { ( \lambda _ { a } \lambda _ { b } ) ^ { n - m + 1 } e ^ { - ( \lambda _ { a } + \lambda _ { b } ) } } { ( \lambda _ { a } + \xi _ { k } ) ( \lambda _ { b } + \xi _ { k } ) } \left[\kappa_{i-1}^{(n-m)}\left(\lambda_{a}\right)^{2} \kappa_{j-1}^{(n-m)}\left(\lambda_{b}\right)^{2}\right.\right. \\
& \left.\left.-\kappa_{i-1}^{(n-m)}\left(\lambda_{a}\right) \kappa_{j-1}^{(n-m)}\left(\lambda_{a}\right) \kappa_{i-1}^{(n-m)}\left(\lambda_{b}\right) \kappa_{j-1}^{(n-m)}\left(\lambda_{b}\right)\right] d \lambda_{b} d \lambda_{a}\right\}
\end{aligned}
$$

where $\Phi$ is defined after (4.25). The integrals in (4.29) can be split into two parts, such that

$$
\begin{aligned}
Q_{k} & =S_{k}^{2}+\sum_{i=1}^{m} \sum_{\substack{j=1 \\
j \neq i}}^{m} \Phi\left\{\int_{0}^{\infty} \int_{0}^{\infty}\left(\frac{e^{-\lambda_{a}} \lambda_{a}^{n-m+1}}{\lambda_{a}+\xi_{k}}\right)\left(\frac{e^{-\lambda_{a}} \lambda_{a}^{n-m+1}}{\lambda_{a}+\xi_{k}}\right)\right. \\
& \times\left[\kappa_{i-1}^{(n-m)}\left(\lambda_{a}\right)^{2} \kappa_{j-1}^{(n-m)}\left(\lambda_{b}\right)^{2}\right] d \lambda_{b} d \lambda_{a}-\int_{0}^{\infty} \int_{0}^{\infty}\left(\frac{e^{-\lambda_{a}} \lambda_{a}^{n-m+1}}{\lambda_{a}+\xi_{k}}\right)\left(\frac{e^{-\lambda_{b}} \lambda_{b}^{n-m+1}}{\lambda_{b}+\xi_{k}}\right) \\
& \left.\times\left[\kappa_{i-1}^{(n-m)}\left(\lambda_{a}\right) \kappa_{j-1}^{(n-m)}\left(\lambda_{a}\right) \kappa_{i-1}^{(n-m)\left(\lambda_{b}\right)} \kappa_{j-1}^{(n-m)}\left(\lambda_{b}\right)\right] d \lambda_{b} d \lambda_{a}\right\} .
\end{aligned}
$$

As the double integrals in (4.30) are of the same function with different variables, 
one can write (4.30) by squaring the result of a single integral, such that

$$
\begin{aligned}
& Q_{k}=S_{k}^{2}+\sum_{i=1}^{m} \sum_{\substack{j=1 \\
j \neq i}}^{m} \Phi\left\{\left[\sum_{f=0}^{i-1} \sum_{\substack{z=0 \\
z \neq f}}^{i-1}(-1)^{f+z}\left(\kappa_{f}\right)\left(\kappa_{z}\right) \frac{1}{f ! z !} \int_{0}^{\infty} \frac{e^{-\lambda} \lambda^{n-m+1+f+z}}{\lambda+\xi_{k}} d \lambda\right]^{2}\right. \\
& \left.-\left[\sum_{f=0}^{i-1} \sum_{\substack{z=0 \\
z \neq f}}^{i-1}(-1)^{f+z}\left(\kappa_{f}\right)\left(\kappa_{z}\right) \frac{1}{f ! z !} \int_{0}^{\infty} \frac{e^{-\lambda \lambda^{n-m+1+f+z}}}{\lambda+\xi_{k}} d \lambda\right]^{2}\right\} \text {. }
\end{aligned}
$$

Recognizing that the integrals in (4.31) have the same form as $J_{n-m+1+f+z, 1,1}\left(\xi_{k}\right)$ and substituting the solution of (4.8) in (4.31) yields the desired expression.

Lemma 4.3.2. Let $M_{k} \leq L_{k}$, where $\theta_{1}, \ldots, \theta_{m}$ are the $m$ eigenvalues of $\boldsymbol{R}_{k} \neq \boldsymbol{I}_{m}$. The expected value of $\left(\sum_{i=1}^{m} \frac{\lambda_{i}}{\lambda_{i}+\xi_{k}}\right)^{2}$ is given by

$$
\begin{aligned}
\bar{Q}_{k}= & \bar{S}_{k}^{2}+\hat{\chi} \sum_{i=0}^{m} \sum_{\substack{j=0 \\
j \neq i}}^{m} \sum_{k=1}^{m} \sum_{\substack{l=1 \\
l \neq k}}^{m}(-1)^{i+j-p(i, j)}(-1)^{k-1+l-p(l)} \operatorname{det}(\boldsymbol{\Xi})_{i, j ; k, l} \\
& \times J_{i+n-m+1,1, \theta_{k}}\left(\xi_{k}\right) J_{j+n-m+1,1, \theta_{l}}\left(\xi_{k}\right),
\end{aligned}
$$

where $\hat{\chi}=\chi(-1)^{\left\lfloor\frac{m}{2}\right\rfloor}(m-2)$ ! with

$$
\chi=\frac{1}{m ! \prod_{l=1}^{m}(n-l) ! \operatorname{det}\left(\boldsymbol{R}_{k}^{n}\right) \prod_{k<l}^{m}\left(\frac{1}{\theta_{l}}-\frac{1}{\theta_{k}}\right)}
$$

and the $(l, k)$-th element of $\boldsymbol{\Xi}$ is given by $e^{-\lambda_{l} / \theta_{k}}$. Moreover,

$$
p(i, j)=\left\{\begin{array}{l}
0 \quad \text { if } i>j \\
1 \quad \text { if } i \leq j
\end{array}\right.
$$

and

$$
p(l)=\left\{\begin{array}{l}
0 \quad \text { if } k>l \\
1 \quad \text { if } k \leq l
\end{array}\right.
$$

and the integrals $J_{i+n-m+1,1, \theta_{k}}\left(\xi_{k}\right)$ and $J_{j+n-m+1,1, \theta_{l}}\left(\xi_{k}\right)$ are defined in (4.8).

Proof of Lemma 4.3.2. To begin, one can substitute the result derived in (2.36) of Chapter 2 into (4.27) where $Q_{k}$ and $S_{k}$ are replaced by their correlated 
central counterparts in $\bar{Q}_{k}$ and $\bar{S}_{k}$. This would result in

$$
\begin{aligned}
\bar{Q}_{k} & =\bar{S}_{k}^{2}+m(m-1)\left[\int_{0}^{\infty} \int_{0}^{\infty}\left(\frac{\lambda_{a}}{\lambda_{a}+\xi_{k}}\right)\left(\frac{\lambda_{b}}{\lambda_{b}+\xi_{k}}\right) \hat{\chi} \sum_{i=0}^{m-1} \sum_{\substack{j=0 \\
j \neq i}}^{m-1}(-1)^{i+j-p(i, j)} \lambda_{1}^{i+n-m} \lambda_{2}^{j+n-m}\right. \\
& \left.\times \sum_{k=1}^{m} \sum_{\substack{l=1 \\
l \neq k}}^{m}(-1)^{k-1} e^{-\lambda_{a} / \theta_{k}}(-1)^{l-p(l)} e^{-\lambda_{2} / \theta_{l}} \operatorname{det}(\boldsymbol{\Xi})_{i, j ; k, l} d \lambda_{b} d \lambda_{a}\right]
\end{aligned}
$$

where $\hat{\chi}, p(i, j), p(l)$ and $\boldsymbol{\Xi}$ are as defined in (4.33) and (4.35). After some mathematical simplifications, one can re-write (4.36) as

$$
\begin{aligned}
\bar{Q}_{k}= & \bar{S}_{k}^{2}+\hat{\chi}\left\{\sum_{i=0}^{m-1} \sum_{\substack{j=0 \\
j \neq i}}^{m-1} \sum_{k=1}^{m} \sum_{\substack{l=1 \\
l \neq k}}^{m}(-1)^{i+j-p(i, j)}(-1)^{k-1+l-p(l)} \operatorname{det}(\boldsymbol{\Xi})_{i, j ; k, l}\right. \\
& \left.\times\left[\int_{0}^{\infty} \frac{e^{-\lambda_{a} / \theta_{k}} \lambda_{a}^{i+n-m+1}}{\lambda_{a}+\xi_{k}} d \lambda_{a} \int_{0}^{\infty} \frac{e^{-\lambda_{b} / \theta_{l}} \lambda_{b}^{j+n-m+1}}{\lambda_{b}+\xi_{k}} d \lambda_{b}\right]\right\}
\end{aligned}
$$

The integrals in (4.37) are of the same form, with varying powers of $i$ and $j$ for $\lambda_{a}$ and $\lambda_{b}$. Their general solution is presented in (4.8). Upon substituting the solution of the integrals in (4.37) yields the expression in Lemma 4.3.2.

Lemma 4.3.3. Let $M_{k}>L_{k}$, where $\theta_{1}, \ldots, \theta_{n}$ are the $n$ eigenvalues of $\boldsymbol{R}_{k} \neq \boldsymbol{I}_{n}$. The expected value of $\left(\sum_{i=1}^{m} \frac{\lambda_{i}}{\lambda_{i}+\xi_{k}}\right)^{2}$ is given by

$$
\begin{aligned}
\tilde{Q}_{k}= & \bar{S}_{k}^{2}+n(n-1) \chi(n-2) !\left\{\sum_{i=0}^{n-1} \sum_{\substack{l=0 \\
l \neq i}}^{n-1} \sum_{o=1}^{n} \sum_{\substack{p=1 \\
p \neq o}}^{n}(-1)^{i+1-p(i, l)}(-1)^{o-1+p-p(i, l)}(-1)^{o-1+p-p(o)}\right. \\
& \left.\times \theta_{o}^{n-m-1} \operatorname{det}\left(\boldsymbol{\Delta}_{n}\right)_{o, p} \operatorname{det}(\boldsymbol{\Xi})_{i, l ; o, p} J_{i+1,1, \theta_{o}}\left(\xi_{k}\right) J_{l+1,1, \theta_{p}}\left(\xi_{k}\right)\right\}
\end{aligned}
$$

The quantities $\chi, p(i, l)$ and $p(o)$ are as given in (4.33) and (4.35). Moreover, $\boldsymbol{\Delta}_{n}$ is an $n \times n$ Vandermonde matrix given by

$$
\boldsymbol{\Delta}_{n}=\left[\begin{array}{cccc}
1 & \theta_{1} & \ldots & \theta_{1}^{n-1} \\
\vdots & \vdots & \ddots & \vdots \\
1 & \theta_{n} & \ldots & \theta_{n}^{n-1}
\end{array}\right]
$$


and $\boldsymbol{\Xi}$ is defined after (4.33).

Proof of Lemma 4.3.3. Following the steps in the proof of Lemma 4.3 .2 and using the joint eigenvalue density derived in (2.46) of Chapter 2 yields the desired expression.

Remark 4.3.3. The generality of the results derived in Theorems 4.3.1, 4.3.2 and Lemmas 4.3.1, 4.3.2 and 4.3.3 is worth mentioning. The results are applicable for any system dimension, link SNR and spatial correlation level. The analysis methodology is also applicable to other channel models (so long as the necessary densities are known), such as i.i.d and semi-correlated Ricean fading. Although an application to two-tier small-cellular networks is considered, the derived results are equally as applicable to classical multi-cellular systems operating with conventional or large antenna arrays.

Remark 4.3.4. The results of Lemmas 4.3 .2 and 4.3 .3 also have further applications to analysis involving complex correlated central Wishart matrices, such as the analysis of second-order statistics of semi-correlated channels, leading to the variance of capacity for such channels.

Using the results derived in Theorems 4.3.1, 4.3.2 and Lemmas 4.3.1, 4.3.2 and 4.3.3, (4.16) can be expressed for i.i.d. and semi-correlated Rayleigh fading channels as

$$
\begin{aligned}
& \varrho_{l, k}^{\text {i.i.d. }}=\frac{Q_{k}+S_{k}^{(2)}}{m(m+1)} \\
& \varrho_{l, k}^{\text {s.c., } M_{k} \leq L_{k}}=\frac{\bar{Q}_{k}+\bar{S}_{k}^{(2)}}{m(m+1)} \\
& \varrho_{l, k}^{\text {s.c., } M_{k}>L_{k}}=\frac{\tilde{Q}_{k}+\bar{S}_{k}^{(2)}}{m(m+1)} .
\end{aligned}
$$

In the same manner, the expected value of the normalization parameter, $\tilde{\eta}_{k}$ for 
cell $k$ can also be stated for i.i.d. and semi-correlated Rayleigh fading as

$$
\begin{aligned}
& \tilde{\eta}_{k}^{\text {i.i.d. }}=\frac{S_{k}^{(1)}}{m} \\
& \tilde{\eta}_{k}^{\text {s.c., } M_{k} \leq L_{k}}=\frac{\bar{S}_{k}^{(1)}}{m} \\
& \tilde{\eta}_{k}^{\text {s.c., } M_{k}>L_{k}}=\frac{\bar{S}_{k}^{(1)}}{m} .
\end{aligned}
$$

Thus, the expected signal power, $\delta_{l, k}$, in (4.14) can be written as

$$
\begin{aligned}
& \delta_{l, k}^{\text {i.i.d. }}=\rho_{\mathrm{dl}, k} \frac{\beta_{k, l, k}}{\tilde{\eta}_{k}^{\text {i.i.d. }}} \varrho_{l, k}^{\text {i.i.d. }} \\
& \delta_{l, k}^{\text {s.c., } M_{k} \leq L_{k}}=\rho_{\mathrm{dl}, k} \frac{\beta_{k, l, k}}{\tilde{\eta}_{k}^{\text {s.c. }, M_{k} \leq L_{k}}} \varrho_{l, k}^{\text {s.c., }, M_{k} \leq L_{k}} \\
& \delta_{l, k}^{\text {s.c. }, M_{k}>L_{k}}=\rho_{\mathrm{dl}, k} \frac{\beta_{k, l, k}}{\tilde{\eta}_{k}^{\text {s.c. }, M_{k}>L_{k}}} \varrho_{l, k}^{\text {s.c. }, M_{k}>L_{k}},
\end{aligned}
$$

for the i.i.d. case and semi-correlated cases, respectively. In the sequel, the expected per-terminal interference power calculations are presented both for i.i.d. and semi-correlated Rayleigh fading.

\section{Expected Per-Terminal Interference Power Analysis}

From (4.12), the expected interference power at the $l$-th terminal in cell $k$ is given by

$\iota_{l, k}=\frac{\rho_{\mathrm{dl}, k} \beta_{k, l, k}}{\tilde{\eta}_{k}}\left\{\sum_{\substack{m=1 \\ m \neq l}}^{L_{k}} \mathbb{E}\left[\left|\boldsymbol{g}_{\mathrm{dl}, k, l, k}^{\mathrm{H}} \boldsymbol{w}_{m, k}\right|^{2}\right]\right\}+\sum_{\substack{j=1 \\ j \neq k}}^{K} \frac{\rho_{\mathrm{dl}, j} \beta_{j, l, k}}{\tilde{\eta}_{j}}\left\{\sum_{q=1}^{L_{j}} \mathbb{E}\left[\left|\boldsymbol{g}_{\mathrm{dl}, j, l, k}^{\mathrm{H}} \boldsymbol{w}_{q, j}\right|^{2}\right]\right\}$.

For network-wide BS coordination, following the methodology of [118], the expected interference power can be evaluated as the difference between the total power (signal and interference) and the desired signal power at the $l$-th terminal in cell $k$. The total power at terminal $l$ is given by

$$
\gamma_{l, k}=\mathbb{E}\left[\left\|\boldsymbol{G}_{\mathrm{dl}, k}^{\mathrm{H}} \boldsymbol{W}_{k}\right\|_{\mathrm{F}}^{2}\right]=\tau L_{k}\left\{\mathbb{E}_{\lambda}\left[\sum_{l=1}^{m}\left(\frac{\lambda_{l}}{\lambda_{l}+\xi_{k}}\right)^{2}\right]\right\}
$$


Subtracting the expected desired signal power in $\delta_{l, k}$ from $\gamma_{l, k}$ yields the interference power at the $l$-th terminal in cell $k$. More specifically, under i.i.d. Rayleigh fading, $\iota_{l, k}$ is given by

$$
\begin{aligned}
\iota_{l, k}^{\text {i.i.d. }}= & \rho_{\mathrm{d} l, k} \frac{\beta_{k, l, k}}{\tilde{\eta}_{k}^{\text {i.i.d. }}}\left[\left(\frac{L_{k}-1}{M_{k}-1}\right)\left(\gamma_{l, k}^{\text {i.i.d. }}-\varrho_{l, k}^{\text {i.i.d. }}\right)\right]+ \\
\rho_{\mathrm{dl}, j} & \sum_{\substack{j=1 \\
j \neq k}}^{K} \frac{\beta_{j, l, k}}{\tilde{\eta}_{j}^{\text {i.i.d. }}}\left[\left(\frac{L_{k}}{M_{k}-1}\right)\left(\gamma_{l, j}^{\text {i.i.d. }}-\varrho_{l, j}^{\text {i.i.j. }}\right)\right] .
\end{aligned}
$$

The equivalent expressions for semi-correlated scenarios, $\iota_{l, k}^{M_{k} \leq L_{k}}$ and $\iota_{l, k}^{M_{k}>L_{k}}$, can be obtained by replacing $\tilde{\eta}_{k}^{\text {i.i.d. }}, \tilde{\eta}_{j}^{\text {i.i.d. }}, \gamma_{l, k}^{\text {i.i.d. }}, \gamma_{l, j}^{\text {i.i.d. }}, \varrho_{l, k}^{\text {i.i.d. }}$ and $\varrho_{l, j}^{\text {i.i.d. }}$ with their semi-correlated counterparts for $M_{k} \leq L_{k}$ and $M_{k}>L_{k}$, respectively. With cellwide coordination, the above approach can be used to find the IUI (first term in (4.43)). However, as the RZF processor designed at BS $k$ is independent of the ICI channels, the second term in (4.43) must be evaluated separately, as demonstrated in the following theorem.

Theorem 4.3.3. In the presence of cell-wide coordination, the expected value of ICI (second term of (4.43)) for terminal $l$ in cell $k$ is given by

$$
\mathbb{E}\left[\left|\boldsymbol{g}_{\mathrm{dl}, j, l, k}^{\mathrm{H}} \boldsymbol{w}_{q, j}\right|^{2}\right]=\mathbb{E}\left[\boldsymbol{g}_{\mathrm{dl}, q, j} \boldsymbol{R}_{j} \boldsymbol{g}_{\mathrm{dl}, q, j}^{\mathrm{H}}\right]=\frac{1}{L_{j}} \sum_{i=1}^{M_{j}} \theta_{i}^{2} f_{i}^{2},
$$

where $\theta_{i}$ is the $i$-th eigenvalue of $\boldsymbol{R}_{j}$ and $f_{i}$ for $i=1, \ldots, M_{j}$ is derived further in the text.

Proof of Theorem 4.3.3. Firstly, one can recognize that $\mathbb{E}\left[\left|\boldsymbol{g}_{\mathrm{dl}, j, l, k}^{\mathrm{H}} \boldsymbol{w}_{q, j}\right|^{2}\right]=$ $\mathbb{E}\left[\boldsymbol{w}_{q, j} \boldsymbol{R}_{j} \boldsymbol{w}_{q, j}^{\mathrm{H}}\right]=\operatorname{tr}\left\{\boldsymbol{R}_{j} \mathbb{E}\left[\boldsymbol{w}_{q, j} \boldsymbol{w}_{q, j}^{\mathrm{H}}\right]\right\}$. Then, $\mathbb{E}\left[\boldsymbol{w}_{q, j} \boldsymbol{w}_{q, j}^{\mathrm{H}}\right]=\frac{1}{L_{j}} \mathbb{E}\left[\boldsymbol{W}_{j} \boldsymbol{W}_{j}^{\mathrm{H}}\right]$, as the columns of $\boldsymbol{W}_{j}$ are identically distributed. Expressing $\boldsymbol{W}_{j}=\boldsymbol{G}_{\mathrm{dl}, j}\left(\boldsymbol{G}_{\mathrm{dl}, j}^{\mathrm{H}} \boldsymbol{G}_{\mathrm{dl}, j}+\xi_{j} \boldsymbol{I}_{L_{j}}\right)^{-1}$, one can denote $\boldsymbol{V}_{j}=\boldsymbol{H}_{\mathrm{dl}, j} \boldsymbol{\Phi}_{j}$ and decompose $\boldsymbol{R}_{j}=\phi_{j} \boldsymbol{\theta}_{j} \boldsymbol{\phi}_{j}^{\mathrm{H}}$, where $\boldsymbol{\theta}_{j}=$ $\operatorname{diag}\left(\theta_{1}, \ldots, \theta_{M_{j}}\right)$ giving

$$
\mathbb{E}\left[\boldsymbol{w}_{q, j} \boldsymbol{R}_{j} \boldsymbol{w}_{q, j}^{\mathrm{H}}\right]=\frac{1}{L_{j}} \operatorname{tr}\left\{\boldsymbol{\phi}_{j}^{\mathrm{H}} \boldsymbol{R}_{j}^{2} \boldsymbol{\phi}_{j} \mathbb{E}\left[\boldsymbol{V}_{j}\left(\boldsymbol{V}_{j} \boldsymbol{\theta}_{j} \boldsymbol{V}_{j}^{\mathrm{H}}+\xi_{j} \boldsymbol{I}_{L_{j}}\right)^{-2} \boldsymbol{V}_{j}\right]\right\}
$$

Noting that the expectation in (4.47) results in a diagonal matrix containing 
$f_{1}, \ldots, f_{M_{j}}$ yields the desired expression in (4.46), where $f_{i}$ is derived in the sequel.

Calculation of $f_{i}$ in $(4.46)$

Denoting $\boldsymbol{V}_{j}=\left[\boldsymbol{v}_{1}, \ldots, \boldsymbol{v}_{M_{j}}\right]$, one can recognize that

$$
f_{i}=\mathbb{E}\left[\boldsymbol{v}_{i}\left(\boldsymbol{V}_{j} \boldsymbol{\theta}_{j} \boldsymbol{V}_{j}^{\mathrm{H}}+\xi_{j} \boldsymbol{I}_{L_{j}}\right)^{-2} \boldsymbol{v}_{i}\right]=-\frac{\partial}{\partial \xi_{j}} \mathbb{E}\left[\boldsymbol{v}_{i}^{\mathrm{H}}\left(\boldsymbol{V}_{j} \boldsymbol{\theta}_{j} \boldsymbol{V}_{j}^{\mathrm{H}}+\xi_{j} \boldsymbol{I}_{L_{j}}\right)^{-1} \boldsymbol{v}_{i}\right]
$$

using a known result from matrix differentiation. Invoking the rank-1 adjustment formula [18], one can obtain $f_{i}=-\frac{\partial}{\partial \xi_{j}} \mathbb{E}\left[\frac{\boldsymbol{X}_{i}}{1+\boldsymbol{X}_{i} \theta_{i}}\right]$, where

$$
\boldsymbol{X}_{i}=\boldsymbol{v}_{i}^{\mathrm{H}}\left[\left(\boldsymbol{V}_{j}\right)_{. ; i}(\boldsymbol{\theta})_{i ; i}\left(\left(\boldsymbol{V}_{j}\right)_{. ; i}\right)^{\mathrm{H}}\right]^{-1} \boldsymbol{v}_{i}
$$

Now, $\boldsymbol{X}_{i}$ is exactly the SINR of a MMSE combiner studied in [124]. Denoting $\bar{F}_{X_{i}}\left(x_{i}\right)$ as the complimentary CDF of $\boldsymbol{X}_{i}, f_{i}=-\frac{\partial}{\partial \xi_{j}} \int_{0}^{\infty} \frac{\bar{F}_{X_{i}}\left(x_{i}\right)}{1+\theta_{i} x_{i}} d x_{i}$. Since $\bar{F}_{X_{i}}\left(x_{i}\right)$ is given in [124], $f_{i}$ can be found by routine integration followed by differentiation w.r.t. $\xi_{j}$. Denoting $(\boldsymbol{\theta})_{i ; i}=\operatorname{diag}\left[\theta_{1}^{(i)}, \ldots, \theta_{M_{j}-1}^{(i)}\right]$, one can write

$f_{i}=\frac{\tilde{\tau}_{m_{1}} \xi_{j}^{m_{1}-1}}{\left(m_{1}-1\right) !} I_{i, m_{1}+1}\left(\xi_{j}\right)-\sum_{l=m_{1}+1}^{L_{j}} \frac{\tilde{\tau}_{l}}{(l-1) ! \operatorname{det}\left(\boldsymbol{\Delta}_{0}\right)}\left[(l-1) \xi_{j}^{l-2} \operatorname{det}\left(\boldsymbol{\Delta}_{1}\right)+\xi_{j}^{l-1} \operatorname{det}\left(\boldsymbol{\Delta}_{2}\right)\right]$,

where

$$
m_{1}=L_{j}-M_{j}, \tilde{\tau}_{l}= \begin{cases}1 & \text { if } l \geq 1 \\ 0 & \text { otherwise }\end{cases}
$$

and

$$
I_{i, m_{1}+1}\left(\xi_{j}\right)=e^{\xi_{j} / \theta_{i}} \sum_{s=0}^{m_{1}-1}\left(\begin{array}{c}
m_{1}-1 \\
s
\end{array}\right) \frac{(-1)^{m_{1}-1-s}}{\theta_{i}^{i+1-s} \xi_{j}^{s-1}} J^{(2)}\left(\frac{\xi_{j}}{\theta_{i}}\right)
$$

respectively. Here, $J^{(2)}\left(\frac{\xi_{j}}{\theta_{i}}\right)$ is as defined in (4.10). Moreover, when $r \neq M_{j}-$ $L_{j}+l-2$,

$$
\left(\boldsymbol{\Delta}_{0}\right)_{r, s}=\left(\theta_{s}^{(i)}\right)^{r-1} \text { and }\left(\boldsymbol{\Delta}_{1}\right)_{r, s}=\left(\boldsymbol{\Delta}_{2}\right)_{r, s}=\left(\boldsymbol{\Delta}_{0}\right)_{r, s}
$$


while when $r=M_{j}-L_{j}+l-2$,

$$
\left(\boldsymbol{\Delta}_{1}\right)_{r, s}=\left(\theta_{s}^{(i)}\right)^{r} \int_{0}^{\infty} \frac{x_{i}^{m_{1}-1} e^{-\xi_{j}} x_{i}}{\left(1+\theta_{i} x_{i}\right)^{2}\left(1+\theta_{s}^{(i)} x_{i}\right)} d x_{i}
$$

and

$$
\left(\boldsymbol{\Delta}_{2}\right)_{r, s}=-\left(\theta_{s}^{(i)}\right)^{r} \int_{0}^{\infty} \frac{x_{i}^{m_{1}} e^{-\xi_{j}} x_{i}}{\left(1+\theta_{i} x_{i}\right)^{2}\left(1+\theta_{s}^{(i)} x_{i}\right)} d x_{i} .
$$

Here, both the integrals have a closed-form solutions via partial fraction decomposition [63]. This concludes the proof.

\section{Expected Per-Terminal SINR and Ergodic Per-Cell Spectral Efficiency}

The expected SINR in (4.12) can now be written as a function of $\delta_{l, k}, \iota_{l, k}, \tilde{\eta}_{k}$ and $\tilde{\eta}_{j}$. That is,

$$
\begin{aligned}
& \mathbb{E}\left[\operatorname{SINR}_{\mathrm{dl}, l, k}\right] \approx \frac{\delta_{l, k}^{\text {i.i.d. }}}{\sigma_{\mathrm{dl}, l, k}^{2}+\iota_{l, k}^{\text {i.i.d. }}} \\
& \mathbb{E}\left[\operatorname{SINR}_{\mathrm{dl}, l, k}^{\text {s.c. } M_{k} \leq L_{k}}\right] \approx \frac{\delta_{l, k}^{\text {s.c. } M_{k} \leq L_{k}}}{\sigma_{\mathrm{dl} l, l, k}^{2}+\iota_{l, k}^{\text {s.c. } M_{k} \leq L_{k}}} \\
& \mathbb{E}\left[\operatorname{SINR}_{\mathrm{dl}, l, l, k}^{\text {s.c. } M_{k}>L_{k}}\right] \approx \frac{\delta_{l, k}^{\text {s.c. } M_{k}>L_{k}}}{\sigma_{\mathrm{dl}, l, k}^{2}+\iota_{l, k}^{\text {s.c. } M_{k}>L_{k}}},
\end{aligned}
$$

for the i.i.d. and semi-correlated cases, respectively. The expected SINRs can be translated into an approximation for the ergodic spectral efficiency of cell $k$ by following (4.13), giving

$$
\mathbb{E}\left[\mathrm{R}_{\mathrm{sum}, \mathrm{dl}, k}\right] \approx \sum_{l=1}^{L_{k}} \log _{2}\left(1+\mathbb{E}\left[\operatorname{SINR}_{\mathrm{dl}, l, k}\right]\right)
$$

for the i.i.d. and equivalently for the semi-correlated scenarios. Having derived the expected SINR and ergodic sum spectral efficiency approximations, their accuracy is evaluated with the BS coordination strategies discussed in Section 4.2.3, for a two-tier small-cellular network detailed in Section 4.5. 


\subsection{High SNR ZF Approximation}

In this section, new analytical results are derived for the expected per-terminal SINR and ergodic per-cell sum spectral efficiency considering unequal spatial correlation matrices for each terminal. More specifically, the high link SNR regime is considered, where the convergence of RZF precoding to ZF precoding is exploited. Here the expected RZF SINR and ergodic sum spectral efficiency is approximated with expected ZF SNR and ergodic sum spectral efficiency. Focusing on the case of network-wide coordination, the spatial correlation matrices follow the model in [120], which considers an exponential structure with a complex correlation co-efficient, $\varphi$, where $|\varphi|$ (magnitude of $\varphi$ ) captures the effects of inter-element spacing at the BS and a unique phase, assumed uniform on $[a, b]$, some subset of $[0,2 \pi]$ is used to differentiate the terminals. The channel from BS $k$ to terminal $l$ in cell $j$ with a terminal specific correlation matrix is given by $\boldsymbol{g}_{\mathrm{dl}, k, l, j}^{\mathrm{H}}=\boldsymbol{h}_{\mathrm{dl}, k, l, j}^{\mathrm{H}} \boldsymbol{R}_{k, l, j}^{\frac{1}{2}}$, as defined in Section 4.2.1.

With network-wide coordination, the $\sum_{i=1}^{K} L_{i} \times M_{k}$ composite channel matrix, $\boldsymbol{G}_{\mathrm{dl}, k}^{\mathrm{H}}=\left[\boldsymbol{Z}_{\mathrm{dl}, 1}, \boldsymbol{Z}_{\mathrm{dl}, 2}, \ldots, \boldsymbol{Z}_{\mathrm{dl}, K}\right]$, where $\boldsymbol{Z}_{\mathrm{dl}, k}$ denotes the downlink channel to all terminals in cell $k$, given by $\boldsymbol{Z}_{\mathrm{dl}, k}=\left[\boldsymbol{g}_{\mathrm{dl}, k, 1, k}, \ldots, \boldsymbol{g}_{\mathrm{dl}, k, L_{k}, k}\right]$. The corresponding $M_{k} \times L_{k}$ un-normalized ZF precoding matrix is defined as $\boldsymbol{W}_{k}=$ $\left[\boldsymbol{G}_{\mathrm{dl}, k}\left(\boldsymbol{G}_{\mathrm{dl}, k}^{\mathrm{H}} \boldsymbol{G}_{\mathrm{dl}, k}\right)^{-1}\right]_{X: Z}$ for the $L_{k}$ terminals located in cell $k$ with $X=\sum_{i=1}^{k-1} L_{i}+$ 1 and $Z=\sum_{i=1}^{k} L_{i}$. The notation $[\boldsymbol{A}]_{X: Z}$ denotes columns $X$ to $Z$ of $\boldsymbol{A}$. The ZF matrix for cell $k$ is normalized by $\eta_{k}=\frac{1}{L_{k}}\left\|\boldsymbol{W}_{k}\right\|_{\mathrm{F}}^{2}$. As $\boldsymbol{G}_{\mathrm{dl}, k}^{\mathrm{H}} \boldsymbol{W}_{k}=$ $\left[\boldsymbol{G}_{\mathrm{dl}, k}^{\mathrm{H}} \boldsymbol{G}_{\mathrm{dl}, k}\left(\boldsymbol{G}_{\mathrm{dl}, k}^{\mathrm{H}} \boldsymbol{G}_{\mathrm{dl}, k}\right)^{-1}\right]_{X: Z}=\left[0, \ldots, \boldsymbol{I}_{X: Z}, \ldots, 0\right]^{\mathrm{T}}$, perfect cancellation of IUI and ICI takes place allowing us to express the received signal at terminal $l$ in cell $k$ as

$$
r_{\mathrm{dl}, l, k}=\rho_{\mathrm{dl}, k}^{\frac{1}{2}}\left(\frac{\beta_{k, l, k}}{\eta_{k}}\right)^{\frac{1}{2}} s_{\mathrm{dl}, l, k}+n_{\mathrm{dl}, l, k},
$$

where $\beta_{k, l, k}, s_{\mathrm{dl}, l, k}$ and $n_{\mathrm{dl}, l, k}$ are as defined in (4.1) and (4.2), respectively. With uniform power allocation and recognizing that $\mathbb{E}\left[\left|s_{\mathrm{dl}, l, k}\right|^{2}\right]=1$, the received ZF 
SNR for terminal $l$ is given by [80]

$$
\mathrm{SNR}_{\mathrm{dl}, l, k}^{\mathrm{ZF}}=\frac{\rho_{\mathrm{dl}, k} \beta_{k, l, k}}{\sigma_{l, k}^{2} \eta_{k}}=\frac{\rho_{\mathrm{dl}, k} \beta_{k, l, k}}{\sigma_{\mathrm{dl}, l, k}^{2}\left\{\operatorname{tr}_{X: Z}\left[\left(\boldsymbol{G}_{\mathrm{dl}, k}^{\mathrm{H}} \boldsymbol{G}_{\mathrm{dl}, k}\right)^{-1}\right]\right\}}
$$

where $\operatorname{tr}_{X: Z}[\boldsymbol{A}]$ represents the trace of the diagonal block of $\boldsymbol{A}$ involving rows and columns $X$ to $Z$. The inverse in (4.58) can be approximated with a classical order $N$ Neumann series (NS), as shown in the analysis methodology of $[57,77$, 78]. Denoting $\boldsymbol{G}_{\mathrm{dl}, k}^{\mathrm{H}} \boldsymbol{G}_{\mathrm{dl}, k}=M_{k} \boldsymbol{I}_{\sum_{i=1}^{K} L_{i}}+\boldsymbol{\Delta}_{k}$, one can write $\boldsymbol{\Delta}_{k}=\boldsymbol{G}_{\mathrm{dl}, k}^{\mathrm{H}} \boldsymbol{G}_{\mathrm{dl}, k}-$ $M_{k} \boldsymbol{I}_{\sum_{i=1}^{K} L_{i}}$ with $\mathbb{E}\left[\boldsymbol{\Delta}_{k}\right]=\mathbf{0}_{L_{i}}$, allowing the inverse to be written as

$$
\left(\boldsymbol{G}_{\mathrm{dl}, k}^{\mathrm{H}} \boldsymbol{G}_{\mathrm{dl}, k}\right)^{-1} \approx \frac{1}{M_{k}} \sum_{p=0}^{N}(-1)^{p}\left(\frac{\boldsymbol{\Delta}_{k}}{M_{k}}\right)^{p}=\frac{1}{M_{k}} \sum_{p=0}^{N} \sum_{i=0}^{p}\left(\begin{array}{l}
p \\
i
\end{array}\right) \frac{(-1)^{i}}{\left(M_{k}\right)^{i}}\left(\boldsymbol{G}_{\mathrm{dl}, k}^{\mathrm{H}} \boldsymbol{G}_{\mathrm{dl}, k}\right)^{i}
$$

Substituting (4.59) into (4.58) yields

$$
\mathrm{SNR}_{\mathrm{dl}, l, k}^{\mathrm{ZF}} \approx \frac{\beta_{k, l, k}}{\sigma_{\mathrm{dl}, l, k}^{2}\left\{\operatorname{tr}_{X: Z}\left[\frac{1}{M_{k}} \sum_{p=0}^{N} \sum_{i=0}^{p}\left(\begin{array}{c}
p \\
i
\end{array}\right) \frac{(-1)^{i}}{\left(M_{k}\right)^{i}}\left(\boldsymbol{G}_{\mathrm{dl}, k}^{\mathrm{H}} \boldsymbol{G}_{\mathrm{dl}, k}\right)^{i}\right]\right\}}
$$

From (4.60), the expected SNR of terminal $l$ in cell $k$ can be approximated as

$$
\mathrm{SNR}_{\mathrm{dl}, l, k}^{\mathrm{ZF}} \approx \frac{\beta_{k, l, k}}{\sigma_{\mathrm{dl} l, l, k}^{2}\left\{\frac{1}{M_{k}} \sum_{p=0}^{n} \sum_{i=0}^{p}\left(\begin{array}{c}
p \\
i
\end{array}\right) \frac{(-1)^{i}}{M_{k}^{i}} \mathbb{E}\left[\operatorname{tr}_{X: Z}\left[\left(\boldsymbol{G}_{\mathrm{dl}, k}^{\mathrm{H}} \boldsymbol{G}_{\mathrm{dl}, k}\right)^{i}\right]\right]\right\}}
$$

using the univariate special case of the first-order Delta expansion motivated in Section 3.4.3 of Chapter 3. In what follows, with a two-term NS (i.e., $N=2$ ), a closed-form expression of (4.61) is derived.

Proposition 4.4.1. When $\boldsymbol{g}_{\mathrm{dl}, k, l, j}^{\mathrm{H}}=\boldsymbol{h}_{\mathrm{dl}, k, l, j}^{\mathrm{H}} \boldsymbol{R}_{k, l, j}^{\frac{1}{2}}$, where $\boldsymbol{h}_{\mathrm{dl}, k, l, j}^{\mathrm{H}} \sim \mathcal{C N}\left(0, \boldsymbol{I}_{M_{k}}\right)$ and $\boldsymbol{R}_{k, l, j}$ is a terminal specific transmit spatial correlation matrix, the RZF expected per-terminal SINR can be approximated with a ZF expected per-terminal SNR with the use of a two-term NS in the high link SNR regime. For the $l$-th 
terminal in cell $k$ being served by BS $k$, this is given by

$$
\mathbb{E}\left[\mathrm{SNR}_{\mathrm{dl}, l, k}^{\mathrm{ZF}}\right] \approx \frac{\beta_{k, l, k}\left(M_{k}\right)^{3}}{\sigma_{\mathrm{dl}, l, k}^{2}\left\{L_{k}\left(M_{k}\right)^{2}+\left\{L_{k} \sum_{k=1}^{K} L_{k} \operatorname{tr}_{X: Z}\left[\overline{\boldsymbol{R}} \overline{\boldsymbol{R}}_{k}\right]\right\}\right\}}
$$

where $\overline{\boldsymbol{R}}=\frac{\sum_{j=1}^{K} \sum_{l=1}^{L_{j}} \boldsymbol{R}_{k, l, j}}{\sum_{j=1}^{K} L_{j}}$ is the average correlation matrix of all terminals in the system and $\overline{\boldsymbol{R}}_{k}=\frac{\sum_{l=1}^{L_{k}} \boldsymbol{R}_{k, l, k}}{L_{k}}$ is the average correlation matrix for the terminals in cell $k$, respectively.

Method for Proposition 4.4.1. From (4.60), when $N=2$, one can write

$$
\begin{aligned}
& \left(\boldsymbol{G}_{\mathrm{dl}, k}^{\mathrm{H}} \boldsymbol{G}_{\mathrm{dl}, k}\right)^{-1}= \\
& \frac{1}{M_{k}}\left\{\boldsymbol{I}_{\sum_{i=1}^{K} L_{i}}-\frac{\boldsymbol{G}_{\mathrm{dl}, k}^{\mathrm{H}} \boldsymbol{G}_{\mathrm{dl}, k}}{M_{k}}+\boldsymbol{I}_{\sum_{i=1}^{K} L_{i}}+\frac{1}{\left(M_{k}\right)^{2}}\left[\left(\boldsymbol{G}_{\mathrm{dl}, k}^{\mathrm{H}} \boldsymbol{G}_{\mathrm{dl}, k}\right)^{2}-2 M_{k} \boldsymbol{G}_{\mathrm{dl}, k}^{\mathrm{H}} \boldsymbol{G}_{\mathrm{dl}, k}+M_{k}^{2} \boldsymbol{I}_{\sum_{i=1}^{K} L_{i}}\right]\right\} \\
& =\frac{1}{M_{k}}\left[3 \boldsymbol{I}_{\sum_{i=1}^{K} L_{i}}-\frac{3}{M_{k}} \boldsymbol{G}_{\mathrm{dl}, k}^{\mathrm{H}} \boldsymbol{G}_{\mathrm{dl}, k}+\frac{1}{\left(M_{k}\right)^{2}}\left(\boldsymbol{G}_{\mathrm{dl}, k}^{\mathrm{H}} \boldsymbol{G}_{\mathrm{dl}, k}\right)^{2}\right] .
\end{aligned}
$$

Taking the partial trace of (4.63) yields

$$
\operatorname{tr}_{X: Z}\left[\left(\boldsymbol{G}_{\mathrm{dl}, k}^{\mathrm{H}} \boldsymbol{G}_{\mathrm{dl}, k}\right)^{-1}\right] \approx \frac{1}{M_{k}}\left\{3 L_{k}-\frac{3}{M_{k}} \operatorname{tr}_{X: Z}\left[\boldsymbol{G}_{\mathrm{dl}, k}^{\mathrm{H}} \boldsymbol{G}_{\mathrm{dl}, k}\right]+\frac{1}{\left(M_{k}\right)^{2}} \operatorname{tr}_{X: Z}\left[\left(\boldsymbol{G}_{\mathrm{d} l, k}^{\mathrm{H}} \boldsymbol{G}_{\mathrm{dl}, k}\right)^{2}\right]\right\}
$$

After some simplifications, the expected value of (4.63) can be written as

$$
\begin{aligned}
\mathbb{E}\left[\operatorname{tr}_{X: Z}\left[\left(\boldsymbol{G}_{\mathrm{dl}, k}^{\mathrm{H}} \boldsymbol{G}_{\mathrm{dl}, k}\right)^{-1}\right]\right] \approx \\
\quad \frac{1}{M_{k}}\left\{3 L_{k}-\frac{3}{M_{k}}\left(L_{k} M_{k}\right)+\frac{1}{\left(M_{k}\right)^{2}}\left[L_{k}\left(M_{k}\right)^{2}+L_{k} \sum_{k=1}^{K} L_{k} \operatorname{tr}_{X: Z}\left[\overline{\boldsymbol{R}} \overline{\boldsymbol{R}}_{k}\right]\right]\right\} \\
=\frac{1}{\left(M_{k}\right)^{3}}\left\{L_{k}\left(M_{k}\right)^{2}+L_{k} \sum_{k=1}^{K} L_{k} \operatorname{tr}_{X: Z}\left[\overline{\boldsymbol{R}} \overline{\boldsymbol{R}}_{k}\right]\right\}
\end{aligned}
$$

where $\overline{\boldsymbol{R}}=\frac{\sum_{j=1}^{K} \sum_{l=1}^{L_{j}} \boldsymbol{R}_{k, l, j}}{\sum_{j=1}^{K} L_{j}}$ is the average correlation matrix of all terminals and $\overline{\boldsymbol{R}}_{k}=\frac{\sum_{l=1}^{L_{k}} \boldsymbol{R}_{k, l, k}}{L_{k}}$ is the average correlation matrix for terminals in cell $k$. Substituting (4.65) into (4.58) yields the desired expression in (4.62).

Following (4.56), the expected per-terminal SNR can be easily translated to 
the ergodic per-cell sum spectral efficiency. Thus,

$$
\mathbb{E}\left[\mathrm{R}_{\mathrm{sum}, \mathrm{dl}, k}^{\mathrm{ZF}}\right] \approx \sum_{l=1}^{L_{k}} \log _{2}\left(1+\mathbb{E}\left[\mathrm{SNR}_{\mathrm{dl}, l, k}^{\mathrm{ZF}}\right]\right)
$$

The accuracy of Proposition 4.4.1 and its translation into the ergodic sum spectral efficiency in (4.66) is evaluated in Section 4.5, where performance differences between equal and unequal correlation matrices are also presented.

Remark 4.4.1. Proposition 4.4.1 provides a closed-form approximation to an extremely complex situation where all terminals have unequal correlation matrices. The structure of Theorem 4.4.1 demonstrates the impact of unequal correlation matrices, primarily through $\operatorname{tr}_{X: Z}\left[\overline{\boldsymbol{R}} \overline{\boldsymbol{R}}_{k}\right]$. From here it is straight forward to show that this partial trace tends to maximize as $\overline{\boldsymbol{R}}_{k}$ approaches $\overline{\boldsymbol{R}}$, the case of equal spatial correlation matrices, maximizing the expected noise power, thereby reducing the expected ZF SNR. As a result of this, the SNR performance of equal correlation matrices tend to act as a lower bound on the performance of such system. This fact is proven later in the thesis in Chapter 7 . Moreover, fixing the partial trace in the denominator of Proposition 4.4.1, along with the other propagation parameters, the effects of increasing $M_{k}$ and $L_{k}$ can be readily observed from the expression. Firstly, increasing $M_{k}$ and $L_{k}$ fixed increases the expected signal power cubically, while increasing the expected noise power quadratically, resulting in a net increase in the expected signal power of order $M_{k}$. On the other hand, fixing $M_{k}$ and increasing $L_{k}$ leads to an exponential amplification of the expected noise power by order $1 /\left(L_{k}+K L_{k}^{2}\right)$, while the signal power remains unaltered. ${ }^{15}$

\subsection{Numerical Results and Discussion}

A two-tier small-cellular network is considered, where tier one composes of macro and tier two composes of microcell BSs in the form of small-cells. ${ }^{16}$. Unless otherwise specified, the simulation parameters were been obtained from [125].

\footnotetext{
${ }^{15}$ Further discussion and mathematical justification on the impact of unequal spatial correlation matrices is presented in Chapter 7 .

${ }^{16}$ From this point onward, a small-cellular BS is denoted as a microcell BS, without loss of generality.
} 
Net transmit powers of macrocell $k$ and microcell $j, \rho_{\mathrm{dl}, k}$ and $\rho_{\mathrm{dl}, j}$ are set to $46 \mathrm{dBm}$ and $30 \mathrm{dBm}$, respectively, unless otherwise stated to vary the link SNRs. Large-scale propagation effects of geometric attenuation and shadowfading follow the model in (4.2), where for $d_{0}=10 \mathrm{~m}, v=31.54 \mathrm{~dB}$ [15]. Attenuation exponent for the macrocell is $\alpha_{k}=3.7$ and for the microcell is $\alpha_{j}=3.4$, whilst the shadow-fading standard deviation, $\sigma_{\mathrm{sf}}=8.0 \mathrm{~dB}$. Circular cell radii of $1 \mathrm{~km}$ and $70 \mathrm{~m}$ are assumed for cells $k$ and $j$, respectively. The location of $\mathrm{BS} k$ remains fixed, whilst the location of $\mathrm{BS} j$ varies depending on the scenario considered (discussed later). The placement of BS $j$ is constrained outside a $70 \mathrm{~m}$ exclusion radius from BS $k$. The total number of single-antenna terminals being served by BSs $k$ and $j$ are $L_{k}=5$ and $L_{j}=3$, which are distributed uniformly over the area of the respective cells. The number of serving antennas at BSs $k$ and $j\left(M_{k}\right.$ and $\left.M_{j}\right)$ vary depending on the scenario considered $\left(M_{k}, M_{j}>L_{k}, L_{j}\right.$ or $\left.M_{k}, M_{j} \leq L_{k}, L_{j}\right)$. For the former, $M_{k}=8, M_{j}=4$ and for the latter, $M_{k}=4, M_{j}=2$, respectively. ${ }^{17}$ With equal correlation matrices, the presence of spatial correlation at BSs $k$ and $j$ is modeled with the classical Kronecker model, where $\left(\boldsymbol{R}_{k}\right)_{x, z}=\left(\boldsymbol{R}_{j}\right)_{x, z}=\varphi^{|x-z|}$ for $x, z \in 1, \ldots, M_{k}$, and $1, \ldots, M_{j}$ has an exponential structure, as in [46]. On the other hand, when each terminal is assigned an unequal correlation matrix, the model in [120, 121] is employed where each correlation matrix has a structure proportional to $\varphi^{|x-z|}$ modeling the inter-element spacing and an independent $[0,2 \pi]$ uniformly distributed phase component is multiplied modeling the terminal distribution in a cell. In what follows, the resulting performance of the system with equal and unequal correlation matrices is assessed.

\subsubsection{Simulation Settings}

An arbitrary network of 11 BSs comprising of 1 macro and 10 microcells is considered, unless otherwise specified. Uniform, cell-edge and cell-centric microcell

\footnotetext{
${ }^{17}$ With $M_{k} \leq L_{k}$, BS $k$ tries to serve more terminals than the number of transmit antennas. With such high user loading factors, in practice, cellular systems rely on user scheduling mechanisms to decide the suitable operating conditions.
} 

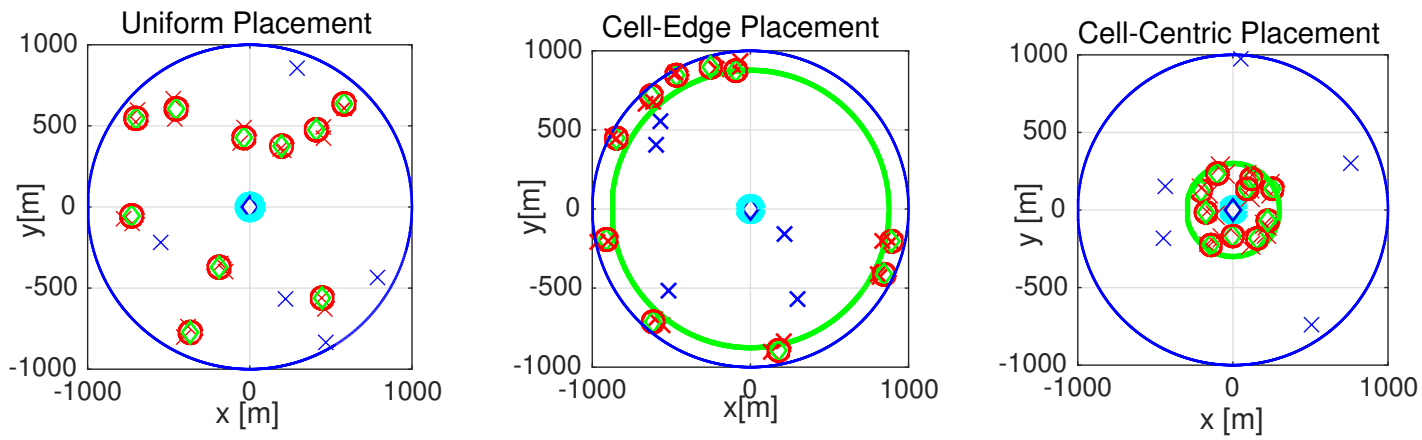

Figure 4.2: Variation in the microcell placement inside the macrocell coverage area.

placements within the macrocell is considered, as shown in Fig. 4.2. For celledge and cell-centric placements, the microcells are restricted such that they are placed outside the cell-edge and inside the cell-centric exclusion areas, marked with green circles in the middle and far-right sub figure of Fig. 4.2. These exclusion zones were numerically determined using the 10-th and 90-th percentiles of the cumulative distribution function (CDF) of the received SNR (defined later) for a typical user terminal associated with the macrocell BS. In particular, the cell-edge of a macrocell was identified as $877 \mathrm{~m}$ to $1000 \mathrm{~m}$ from the center and the cell-center was identified from the origin of the macrocell to $230 \mathrm{~m} .{ }^{18}$ The $70 \mathrm{~m}$ macro exclusion zones are marked with cyan circles in all sub figures of Fig. 4.2. The microcells are denoted with red circles with its associated terminals denoted with red crosses. Likewise, terminals associated to the macro BS are marked with blue crosses. Finally, macro and micro BSs are denoted with blue and green diamonds, respectively.

The results presented in Section 4.5.1 includes an evaluation of the numerically simulated and approximated expected SINR and ergodic sum spectral efficiency derived in (4.12), (4.13), (4.55) and (4.55) for i.i.d., as well as semi-correlated Rayleigh fading having equal correlation matrices. Section 4.5.1 presents numerical performance evaluation of expected SINR and ergodic sum spectral efficiency with unequal correlation matrices using the expressions derived in (4.62) and (4.66). Note that all numerical results are generated using $10^{4}$

\footnotetext{
${ }^{18}$ The cell-edge and cell-centric exclusion zones were calculated from a standard link budget and are sensitive to the chosen numerical parameters.
} 


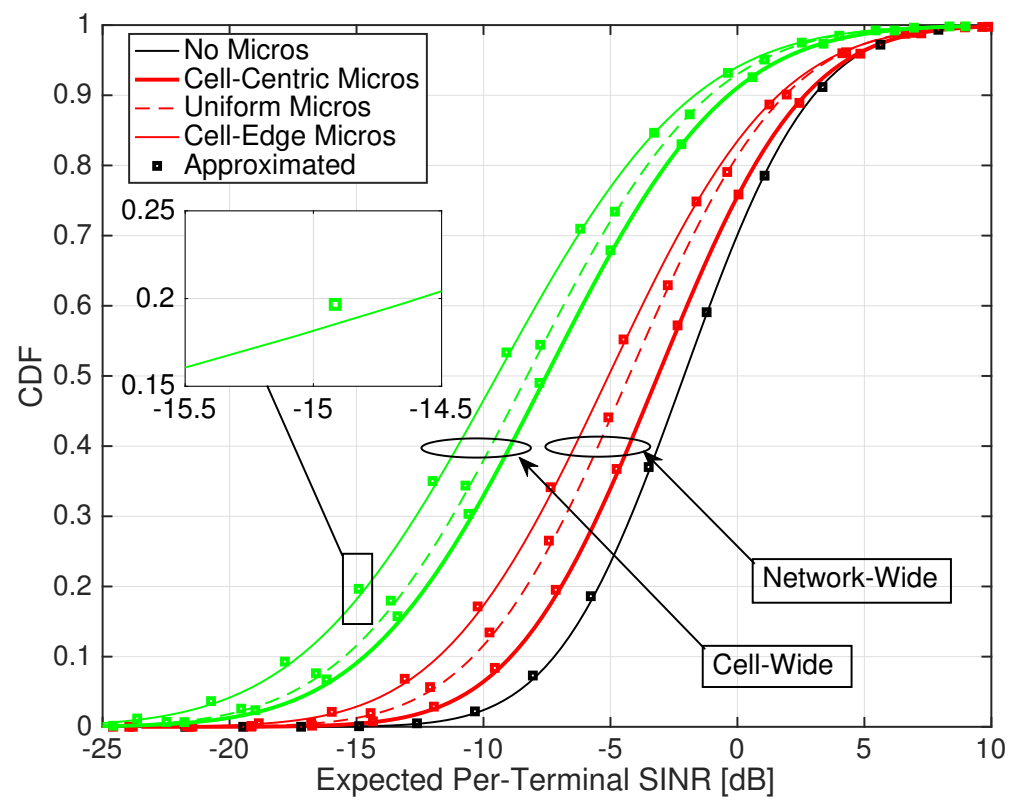

Figure 4.3: Expected per-terminal SINR CDF for a typical terminal associated with the macrocell BS with link $\mathrm{SNR}=10 \mathrm{~dB}, \varphi=0$ and $M \leq L$.

independent trails with the coordination strategies discussed in Section 4.2.3. For each numerical result, $\sigma_{\mathrm{dl} l, l, k}^{2}=\sigma_{\mathrm{dl}}^{2}=1, \forall l, k$.

\section{Tightness of Expected SINR and Ergodic Sum Spectral Efficiency}

Fig. 4.3 shows the expected SINR CDF of a macro terminal with cell-centric, cell-edge and uniform microcell placements at link $\mathrm{SNR}=10 \mathrm{~dB}$ with $\varphi=0$ and $M \leq L$. The expectation is performed over the fast-fading with the distribution representing the randomness in terminal position and shadow-fading. The case with no microcells is considered as a baseline. The cell-centric microcell placement results in the best macro user SINR performance, as the terminals are distributed uniformly over the macrocell coverage area and thus have higher probability of being further away from the micro BSs, resulting in less ICI. This is followed by uniform and cell-edge placements, which often result in closer proximity to a typical macro terminal, having an adverse effect on its SINR. The SINR gains of network-wide coordination relative to cell-wide coordination are more prominent in the lower half of the CDF $(<0.5)$, where the combined 


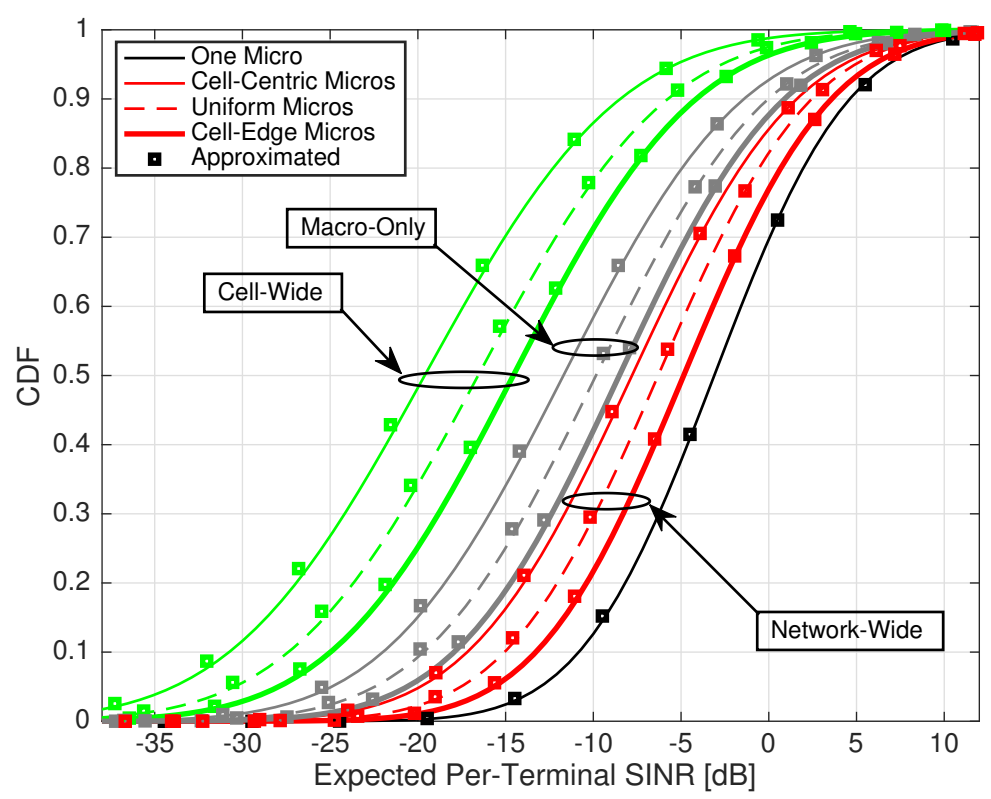

Figure 4.4: Expected per-terminal SINR CDF for a typical terminal associated with the microcell BS with link $\mathrm{SNR}=10 \mathrm{~dB}, \varphi=0$ and $M \leq L$.

effects of noise with IUI and ICI dominate ${ }^{19}$. In contrast, at higher probabilities $(>0.9)$, the spread between the extreme cases of cell-centric microcells with network-wide coordination and cell-edge microcells with cell-wide coordination becomes narrower, due to the reduction in IUI and ICI relative to the signal power. This in-turn suggests that the cell-edge rates of the system $(<0.1)$ may have higher variability than the peak-rates of the system $(>0.9)$ and will benefit more from coordination. It is also observed that in all cases, the derived SINR approximations closely follow the simulated responses over the entire probability range.

Fig. 4.4 shows an equivalent $\mathrm{CDF}$ of a typical micro terminal. Here, the case of a single microcell in the macro coverage area is considered as the baseline. Naturally, all three coordination strategies are applicable to the micro terminal. As expected, the opposite trend to that in Fig. 4.1 is seen, where the cell-edge microcell placement results in superior performance, followed by uniform and

\footnotetext{
${ }^{19}$ For the typical macro terminal, cell-wide and macro-only coordination result in equal performance due to the nature of the respective coordination strategies. Thus, for clarity, the expected SINR distributions with macro-only coordination are omitted.
} 


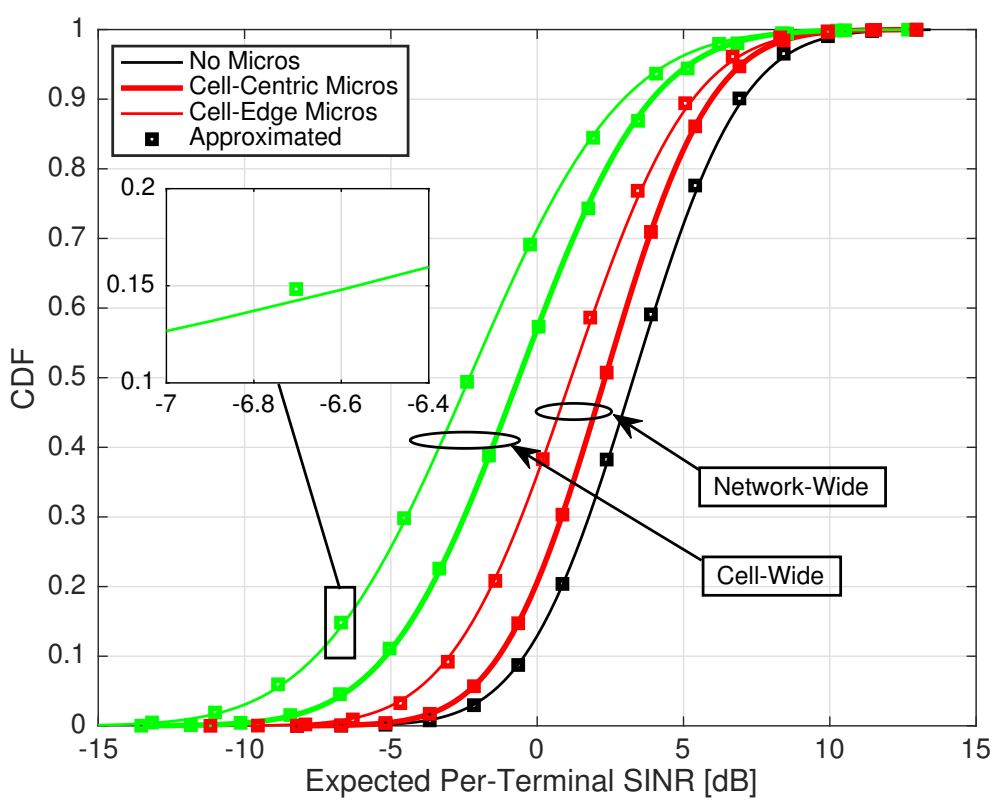

Figure 4.5: Expected per-terminal SINR CDF for a typical terminal associated with the macrocell BS with link SNR $=10 \mathrm{~dB}, \varphi=0$ and $M>L$.

cell-centric placements, where ICI from the macrocell is likely to be higher. At probability 0.5 , irrespective of the microcell placement, macro-only and cell-wide coordination strategies reduce the expected SINR by approximately $4 \mathrm{~dB}$ and 10 $\mathrm{dB}$ relative to network-wide coordination, as the CDFs exhibit parallel behavior. As before, higher variability in the SINRs can be seen at lower probabilities and the derived SINR approximations are seen to remain tight against the simulated equivalents. Now, the case of $M>L$ is considered, where the performance of macro and microcell user terminals is evaluated in Figs. 4.5 and 4.6, respectively. For comparison with Figs. 4.3 and 4.4, all propagation parameters are kept the same. An increase in the expected per-terminal SINR is observed with cellwide, macro-only and network-wide coordination strategies for both the macro and micro terminals ${ }^{20}$. This increase comes at the cost of increasing the spatial d.o.f. (a result of more serving antennas), which allows better ICI control via coordinated RZF. The improvement in the expected per-terminal SINR as a function of link SNR is demonstrated in Fig. 4.7 for a typical macro terminal.

\footnotetext{
${ }^{20}$ Since the trends resulting from the three types of microcell placements are identical for further results considered, to enhance legibility, the subsequent figures only include cell-edge and cell-centric placements.
} 


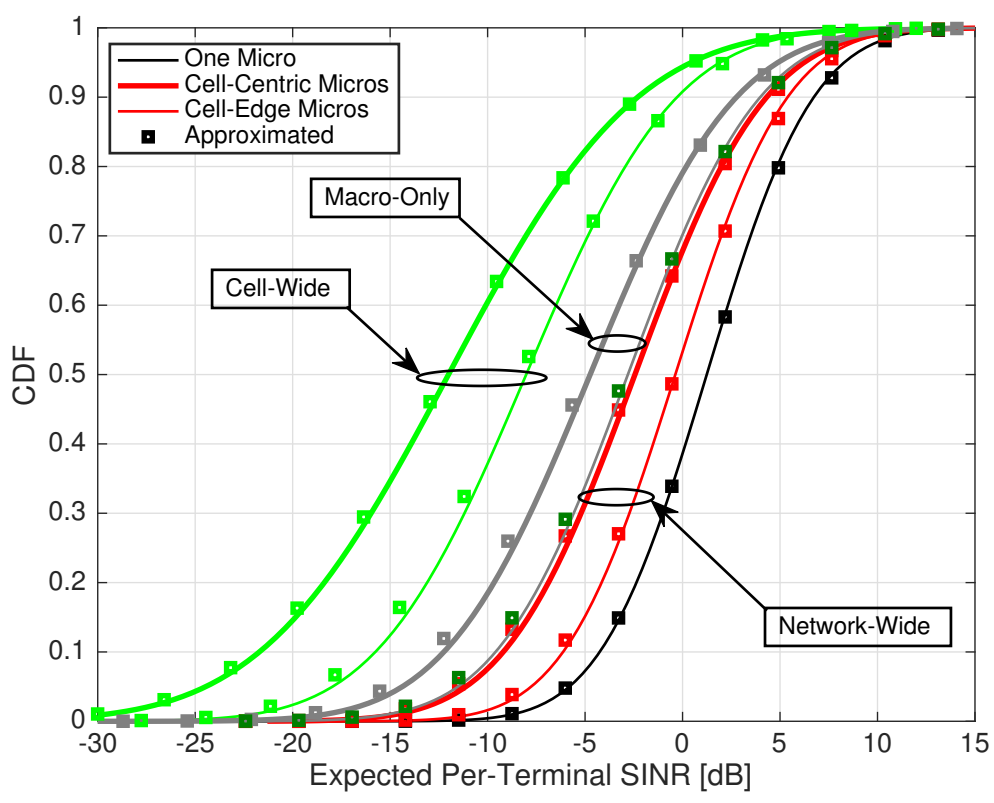

Figure 4.6: Expected per-terminal SINR CDF for a typical terminal associated with the microcell BS with link $\mathrm{SNR}=10 \mathrm{~dB}, \varphi=0$ and $M>L$.

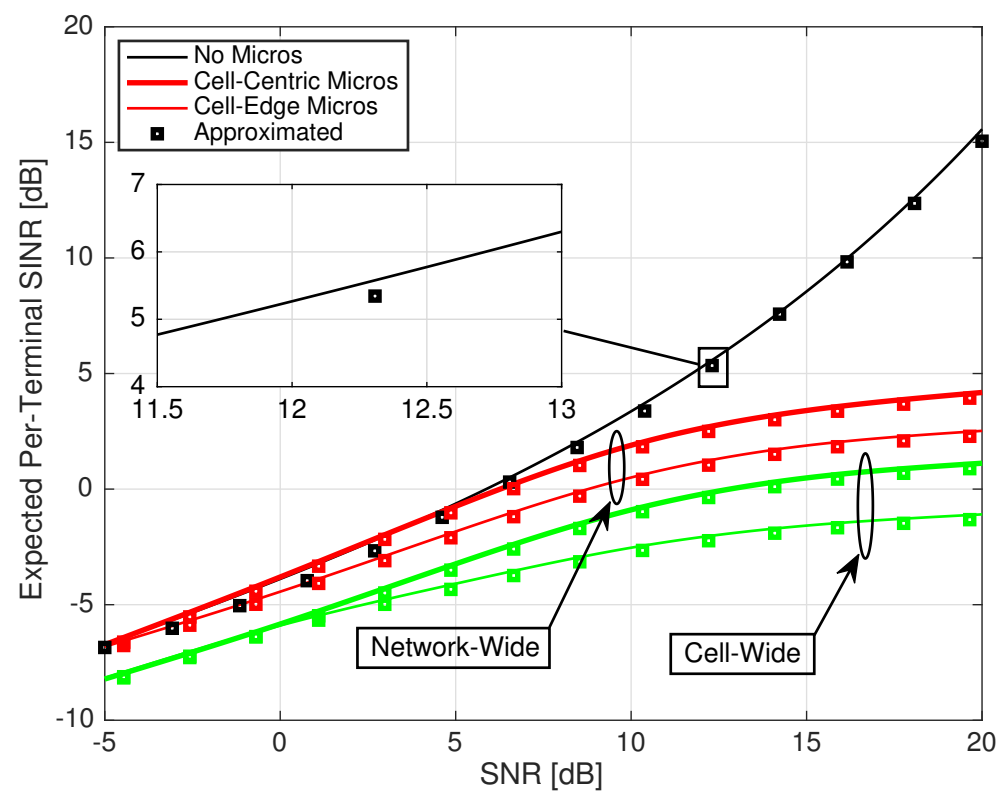

Figure 4.7: Expected per-terminal SINR vs. link SNR for a typical terminal associated with the macrocell BS. Note that $\varphi=0$ and $M>L$ and the expectation is performed globally over the link gains, as well as multipath fading.

The link SNRs are varied by increasing $\rho_{\mathrm{dl}, k}$ for BS $k$. Here, the averaging is performed globally over the link gains, as well as the multipath fading, denoted by $\mathbb{E}_{\beta, \boldsymbol{h}}[\cdot]$. The analytical expressions remain tight throughout the entire link 


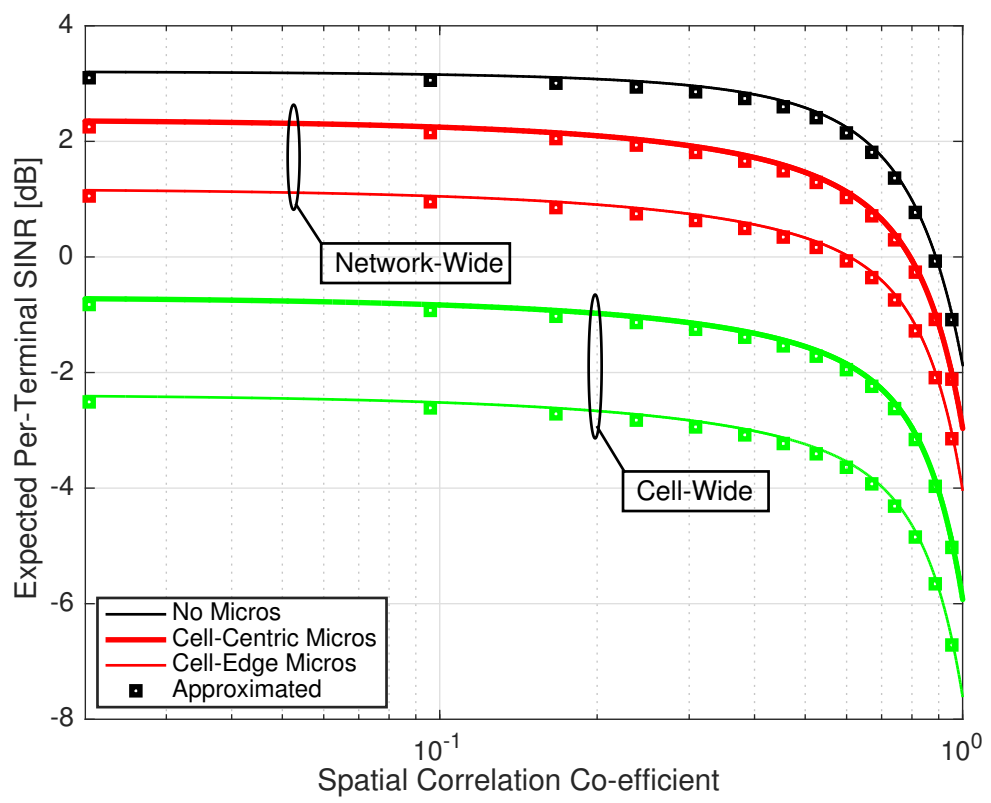

Figure 4.8: Expected per-terminal SINR vs. correlation co-efficient for a typical terminal associated with the macrocell BS. Note that link SNR $=10 \mathrm{~dB}$ and $M>L$. The expectation is performed globally over the link gains, as well as multipath fading.

SNR range considered. The baseline case of no microcells demonstrates a near linear increase in the expected SINR with increasing link SNR, as the serving antennas at the macro BS exceeds the total number of terminals, nulling the IUI. The remaining cases with cell-wide and network-wide coordination still suffer from ICI, saturating the SINR in the high link SNR regime.

Under semi-correlated Rayleigh fading, the expected per-terminal SINR is seen to degrade with increasing levels of spatial correlation at the BS. Fig. 4.8 shows the expected SINR of a macro terminal as a function of spatial correlation, $\varphi$. While varying $\varphi$ from $0-0.6$ has very little effect on the expected SINR, from $\varphi=0.7$ onwards, a heavy penalty in the expected SINR is paid due to a greater reduction in the usable spatial d.o.f. This trend is visible for all microcell placements irrespective of the coordination strategy. The reduction in the spatial d.o.f. can alternatively be interpreted as an increase in the IUI, thus decreasing the per-terminal SINR. Although not shown, the same trend in the degradation of expected per-user SINR can be observed for a micro terminal. 


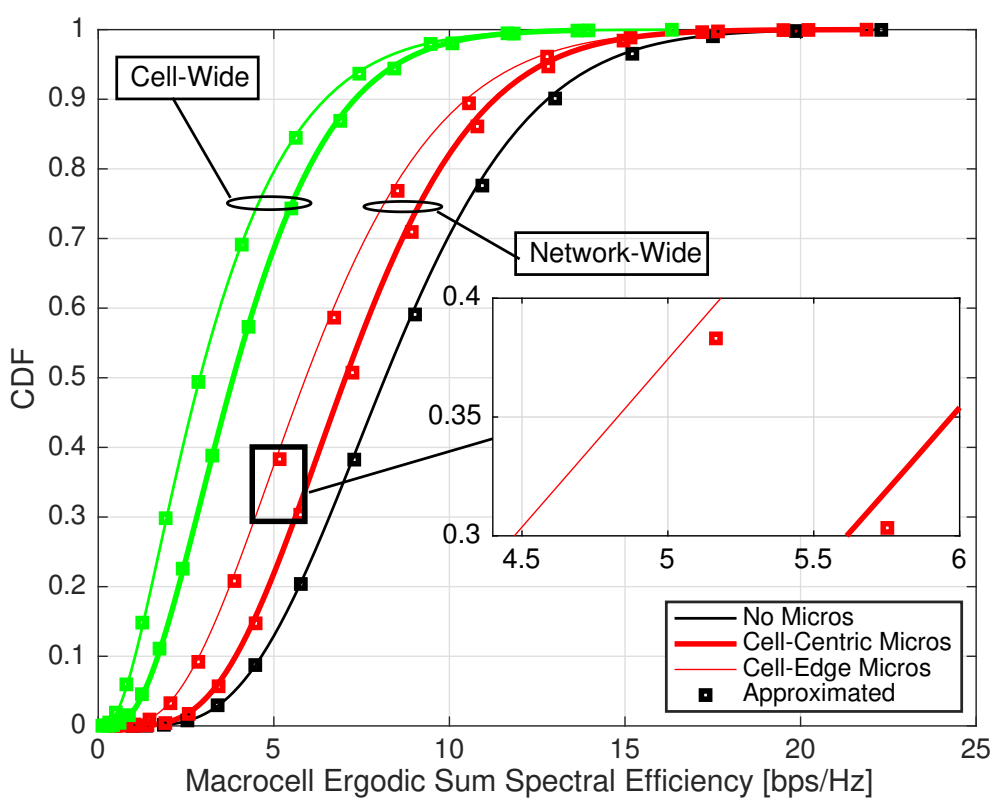

Figure 4.9: Ergodic per-cell sum spectral efficiency for the macrocell at link $\mathrm{SNR}=10 \mathrm{~dB}, M>L$ and $\varphi=0$.

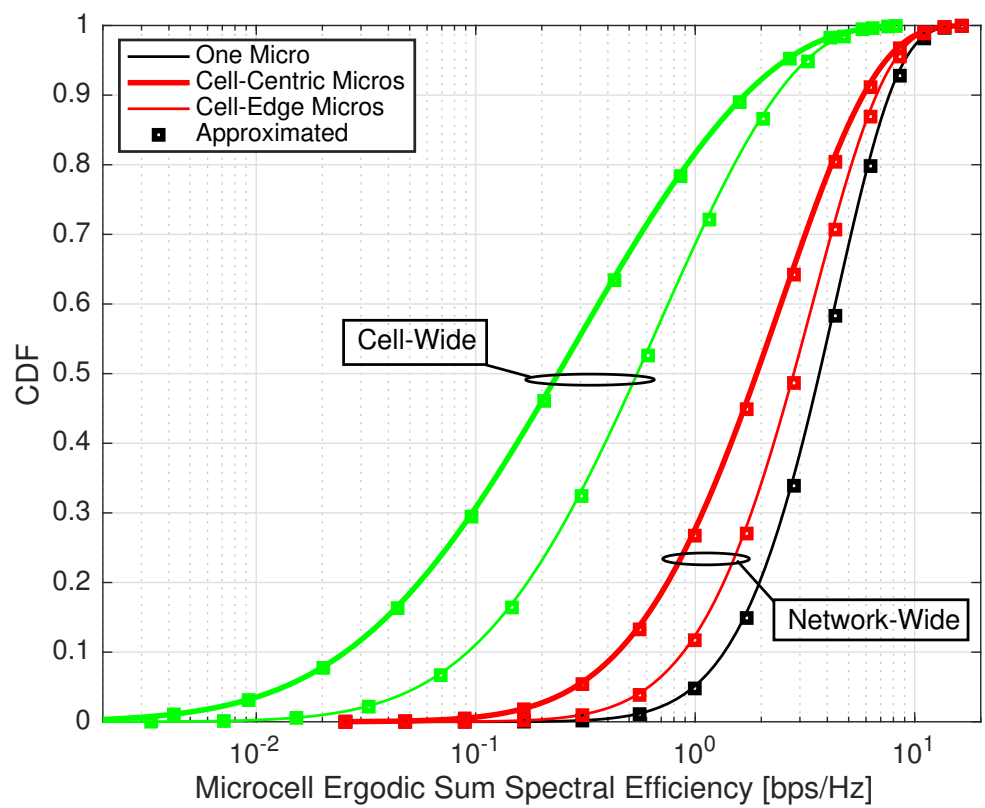

Figure 4.10: Ergodic per-cell sum spectral efficiency for a typical microcell at link $\mathrm{SNR}=10 \mathrm{~dB}, M>L$ and $\varphi=0$.

For all cases, our analytical approximations remain tight even for extremely high levels of channel correlation. The expected SINR distributions of the macro and microcell terminals in Figs. 4.5 and 4.6 were translated into distributions of 


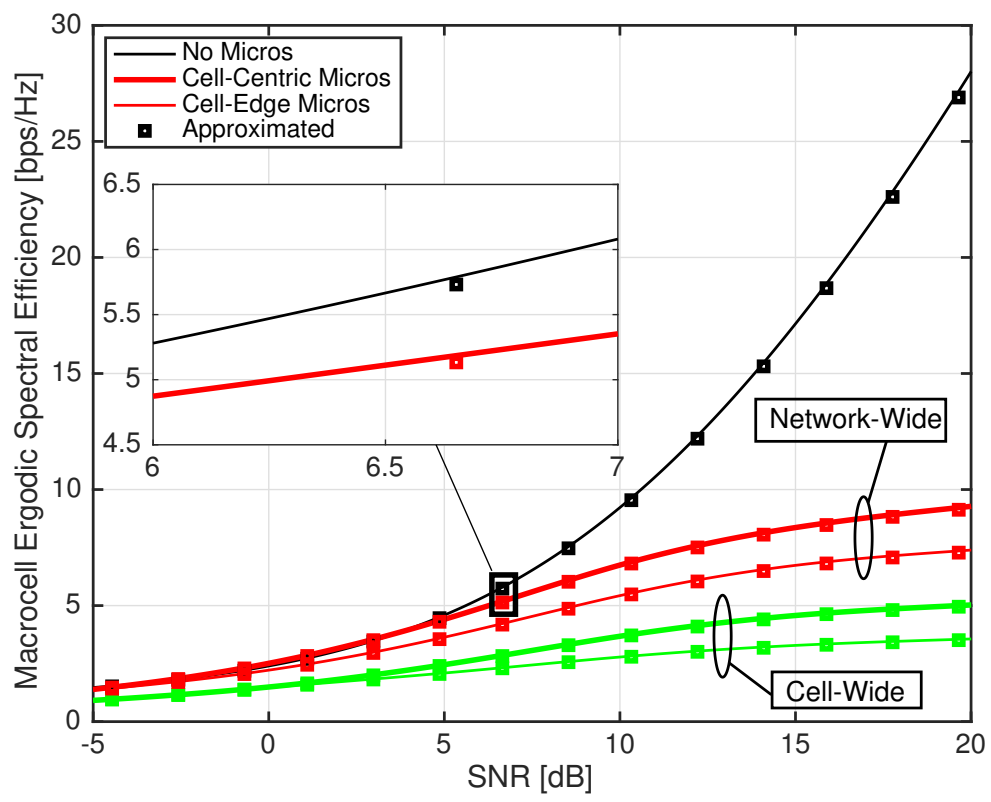

Figure 4.11: Ergodic sum spectral efficiency for a macrocell vs. link SNR $M>L$ and $\varphi=0$.

ergodic per-cell sum spectral efficiencies following (4.13) and (4.56). These are shown in Figs. 4.9 and 4.10 for the macro and a typical microcell, respectively. For the macrocell, higher variability in the peak rates is observed in comparison to cell-edge and median rates, as the combined effect of IUI and ICI impacts performance. Equivalently, for the typical microcell, higher variability in the cell-edge, median and peak rates demonstrates its sensitivity to the aggregate interference and its location within the macrocell. This suggests that the celledge, median and peak-rates will benefit from BS coordination. The macrocell ergodic sum spectral efficiency as a function of the link SNR is shown in Fig. 4.11. Similar trend to Fig. 4.7 is observed, where at high SNRs ICI causes the spectral efficiency to plateau for both the network and cell-wide coordination.

\section{Impact of Unequal Spatial Correlation}

A network of $4 \mathrm{BSs}$ composing of 1 macro and 3 overlaid microcells is considered. Keeping the same constraints in the microcell placements as in the previous subsection, the performance of the system is evaluated with networkwide coordination where each terminal is assigned an unequal correlation matrix 


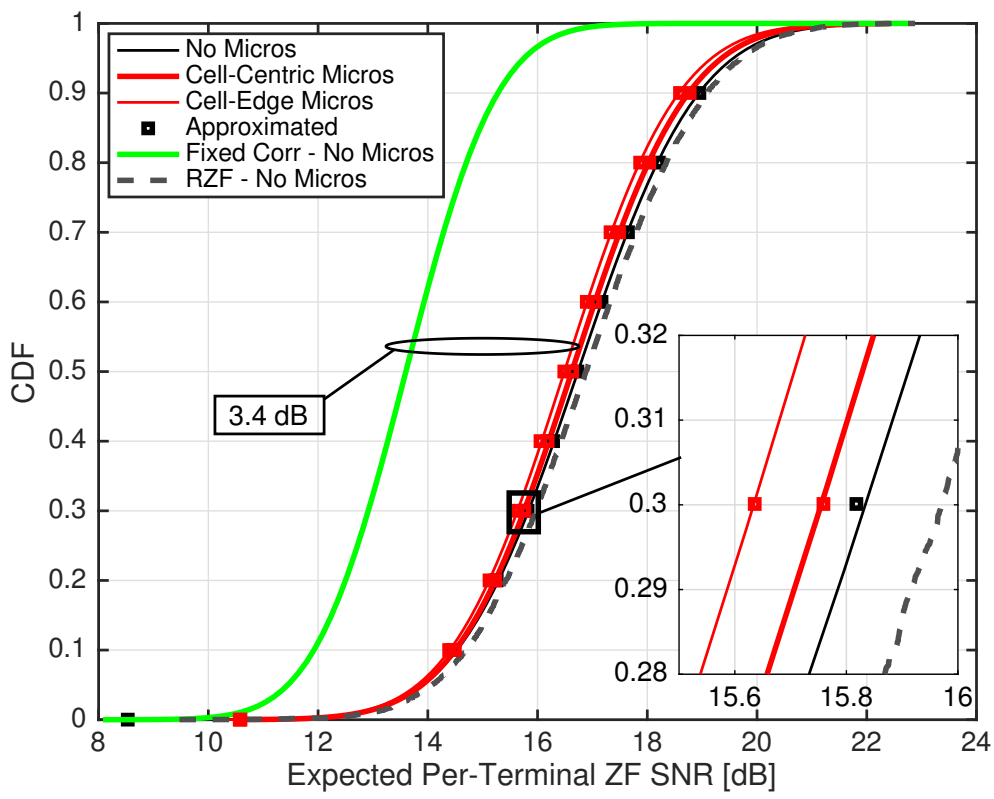

Figure 4.12: CDF of the expected ZF SNR for a typical macrocell terminal with unequal spatial correlation at link $\mathrm{SNR}=10 \mathrm{~dB}$.

capturing the effects of inter-element spacing at the BS and terminal locations in the cell. Following the model described in Section 4.4, for each subsequent result, $|\varphi|=0.9$ with uniformly distributed phase on $[0,2 \pi]$. As ZF precoding is employed, 16 and 10 transmit antennas are considered at macro and micro BSs, serving 3 (macro) and 2 (microcell) terminals. Figs. 4.12 and 4.13 show the expected per-terminal SNR for the typical macro and microcell terminals. Due to zero interference, and the relatively large number of serving antennas in comparison to the number of terminals, a significant increase in the macro and micro terminal SINRs can be observed. In such scenarios, where interference is not the performance limiting factor, the need for coordination is less convincing than in previous cases. For both the macro and micro terminals, varying the microcell placements has a very minor impact on their SINRs. The two-term NS approximations to the expected ZF per-terminal SNR is seen to remain tight for all coordination mechanisms in both Figs. 4.12 and 4.13, respectively. A comparison to the RZF expected per-terminal SINR is made in the baseline cases of no micros and one micro in Figs. 4.12 and 4.13, where ZF expected SNR is shown to closely match the RZF performance. Also, the expected SNR 


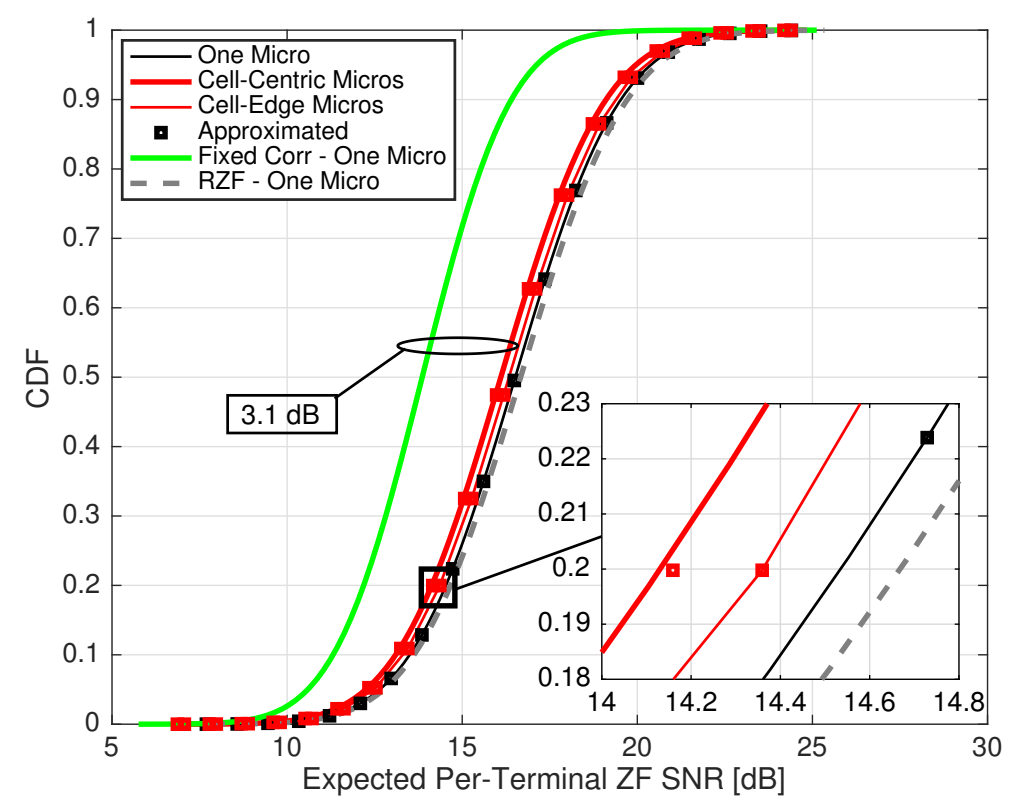

Figure 4.13: CDF of the expected ZF SNR for a typical microcell terminal with unequal spatial correlation at link $\mathrm{SNR}=10 \mathrm{~dB}$.

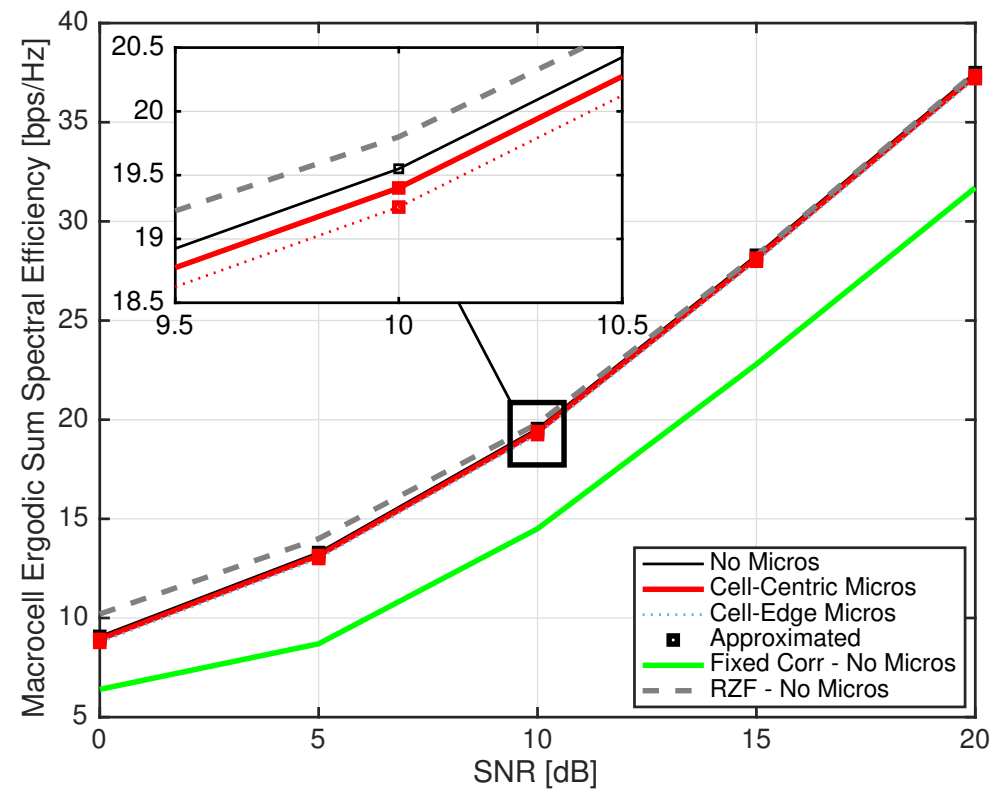

Figure 4.14: Macrocell ergodic sum spectral efficiency vs. link SNR with unequal spatial correlation matrices for each terminal.

performance with unequal correlation matrices is superior in Figs. 4.12 and 4.13 than the case where each terminal has an equal correlation matrix, due to the denominator of (4.62) increasing, as predicted earlier in Remark 4.4.1. This 
gap is seen to translate into the ergodic spectral efficiency for the macrocell and remains approximately constant across the link SNR range considered. This is shown in Fig. 4.14, where the derived approximations retain their tightness and are thus insensitive to changes in the link SNRs.

\subsection{Concluding Remarks}

A general analytical framework for characterizing the expected SINR and ergodic sum spectral efficiency of a multi-cellular system was presented. An application to two-tier small-cellular networks was considered with varying degrees of coordinated RZF processing. Assuming both i.i.d. and semi-correlated Rayleigh fading, with equal correlation matrices, the analytical expressions were averaged over the eigenvalue densities of the respective complex Wishart matrices. In the high link SNR regime, with ZF precoding, closed-form expressions were derived to approximate the RZF expected SINR and ergodic spectral efficiency with unequal correlation matrices. Numerical results demonstrated the tightness of analytical expressions over a wide range of link SNRs, spatial correlation levels and system dimensions. It was observed that the gains in the expected per-terminal SINR and ergodic spectral efficiencies were influenced by microcell locations and varying degrees of BS coordination, as they directly impacted the systems ability to suppress dominant ICI. Under semi-correlated fading, the expected SINR decreased with increasing levels of spatial correlation due to a loss in the usable spatial degrees of freedom. Expected SINR and ergodic spectral efficiencies with unequal correlation matrices were observed to be greater than the case with equal correlation matrices, as predicted mathematically. 


\section{Chapter 5}

\section{RZF Analysis in Ricean Fading}

Summary. With the emergence of small-cellular systems, a serving base station (BS) is more likely to be within the vicinity of a given user terminal. As a result of this, line-of-sight (LoS) in the propagation channel from the BS to the terminal is likely to dominate future wireless access. In this chapter, the impact of a dominant LoS direction on the terminal signal-to-interference-plusnoise-ratio (SINR) and system spectral efficiency is investigated. Statistically, a Ricean fading channel model is employed to model the presence of LoS along with many scattered components in the propagation channel. Considering a multiuser multiple-input multiple-output (MU-MIMO) system, analytical expressions which approximate the expected (average) per-terminal SINR and ergodic sum spectral efficiency are derived. The analysis assumes regularized zero-forcing precoding (RZF) on the downlink. The derived expressions are averaged with respect to the previously unknown arbitrary eigenvalue densities of the complex non-central Wishart distributed instantaneous channel correlation matrix. To aid the derivation of the expected SINR, analytical expressions derived in Chapter 2 for the joint density of two arbitrary eigenvalues of the complex non-central Wishart matrix are employed. Unlike previous studies, a unique Rice factor is modeled for each terminal, making the analysis and evaluation applicable to physical propagation channels with varying LoS strengths as a result of terrain variations across multiple terminals. Furthermore, in the high link signal-to-noise-ratio (SNR) regime, where RZF precoding converges 
to zero-forcing (ZF) precoding, an accurate approximation is developed for the distribution of the instantaneous ZF SNR of a given terminal. It is shown that the ZF SNR is well approximated by the gamma distribution, whose parameter estimates are derived. The presented numerical findings suggest that while the presence of dominant LoS has an adverse effect on the expected RZF SINR, RZF ergodic sum spectral efficiency and instantaneous ZF SNR, increasing the Rice factor variability tends to enhance the peak RZF ergodic sum spectral efficiency and ZF SNR. Furthermore, both the developed approximations are insensitive to changes in the system dimensions, link SNRs and unequal levels of LoS, respectively.

\subsection{Introduction}

With the focus on large antenna arrays at the base station (BS), for measured non-line-of-sight (NLoS) channels, linear pre-processing techniques such as zeroforcing (ZF) precoding have achieved $98 \%$ of optimal dirty paper coding capacity $[75,124]$. However, to overcome noise inflation in the low signal-to-noiseratio (SNR) regime, regularized zero-forcing (RZF) precoding was proposed [118]. Indeed, several works have theoretically characterized the terminal signalto-interference-plus-noise-ratio (SINR) and system spectral efficiency gains of downlink multiuser multiple-input multiple-output (MU-MIMO) systems with RZF precoding (see e.g., $[57,60,116,118]$, as well as the references therein).

However, most works tend to adopt the simple Rayleigh fading model, appropriate for modeling rich scattering environments, where a given user terminal is assumed to be in dense clutter, experiencing a 360 degree angular spread, with the electromagnetic radiation coming in equally from all directions. Nevertheless, Rayleigh fading fails to capture the presence of line-of-sight (LoS), which may be a dominant feature of future wireless systems operating with large antenna arrays in smaller cells. ${ }^{1}$ Therefore, understanding the performance of

\footnotetext{
${ }^{1}$ This could, perhaps, take place in the candidate millimeter-wave (mmWave) frequency bands, where the probability of a given terminal likely to experience LoS is much higher than at microwave frequencies, as demonstrated in [82, 109, 126-133].
} 
MU-MIMO systems operating with dominant LoS conditions is of growing importance. Limited numbers of works have considered the use of Ricean fading channels in the MU-MIMO context $[67,68,111,134,135]$, where the focus has largely been on characterizing the energy and sum spectral efficiency performance of the system. Moreover, for simplicity, [67,68, 134, 135] evaluate the system performance with a fixed Rice factor for each terminal, despite their different geographical locations leading to variations in the terrain across multiple terminals.

In contrast to previous studies (see e.g., [67,68,111,134,135] and references therein), in this chapter, a Ricean fading channel model is considered, where the impact of LoS propagation is examined on the expected per-terminal SINR and ergodic sum spectral efficiency of a MU-MIMO system with downlink RZF precoding. Furthermore, in the high link signal-to-noise-ratio (SNR) regime, when RZF precoding converges to ZF precoding, an approximation to the distribution of the instantaneous RZF SINR is derived with a ZF SNR, for an arbitrary user terminal. It is demonstrated that the ZF SNR is well approximated by the gamma distribution and the necessary shape and scale parameters are derived. Overall, considering both microwave and mmWave channel parameters, a general analysis methodology is developed that is scalable to any system dimension, link SNR level, and LoS heterogeneity. To the best of the author's knowledge, such general treatment of the instantaneous and expected RZF SINR, RZF ergodic sum spectral efficiency and instantaneous ZF SNR has not been carried out previously.

More specifically, the main contributions in the chapter are as follows:

1. Tight analytical expressions are derived to approximate the expected perterminal SINR and ergodic sum spectral efficiency of the system with Ricean fading channels. Expected signal and interference powers are derived by averaging over the appropriate eigenvalue densities of the complex non-central Wishart channel correlation matrix. To the best of the author's knowledge, such an analysis has not been carried out previously. 
2. In the high link SNR regime, a distributional approximation to the instantaneous per-terminal RZF SINR is derived. It is shown that the ZF SNR very accurately approximates the instantaneous RZF SINR with a gamma distribution. The necessary shape and scale parameters of the gamma distribution are derived.

3. The derived analytical expressions are robust to changes in system dimension and considers the important case where each terminal in the system has a unique Rice factor. Previously, this level of accuracy over such a wide range of scenarios has not been achieved. The presented numerical findings suggest that increasing the mean of the Rice factor in both the microwave and mmWave frequency bands has an adverse effect on the instantaneous ZF SNR, expected RZF SINR and ergodic sum spectral efficiency. Furthermore, with a fixed mean, the impact of increased Rice factor variability is demonstrated, where it is shown that the onset of low Rice factors tends to increase the peak performance of the system, as it results in a large occurrence of higher data rates. Interestingly, the opposite effect is observed at the cell-edge, as a highly variable Rice factor tends to draw large values of the Rice factor, degrading the cell-edge performance.

The remainder of the chapter is structured as follows: Section 5.2 details the system and channel model employed for the chapter with downlink RZF precoding. Section 5.3 presents the RZF expected per-terminal SINR and ergodic sum spectral efficiency approximations. Furthermore, Section 5.4 considers the high link SNR regime, and presents a distributional approximation to the instantaneous RZF SINR with a ZF SNR, for a given user terminal. Section 5.5 presents the numerical performance evaluation of the considered system with RZF and ZF precoding, and Section 5.6 presents the concluding remarks of the chapter. 


\subsection{System Model}

The downlink of a single-cell, MU-MIMO system operating in an urban microcell (UMi) environment is considered. The BS is equipped with $M$ transmit antennas configured in a uniform linear array (ULA) to serve $L$ non-cooperative single antenna terminals $(M \geq L)$ in the same time-frequency interval. No mechanical downtilt is assumed with an ideal radiation pattern. In the subsections which follow, the channel model along with the per-terminal SINR and ergodic sum spectral efficiency with RZF precoding is presented.

\subsubsection{Channel Model}

An uncorrelated Ricean fading channel is assumed, where the $1 \times M$ smallscale fading propagation channel between the BS and the $l$-th terminal can be expressed as

$$
\boldsymbol{h}_{\mathrm{dl}, l}^{\mathrm{H}}=\sqrt{\frac{K_{l}}{K_{l}+1}} \overline{\boldsymbol{h}}_{\mathrm{dl}, l}^{\mathrm{H}}+\sqrt{\frac{1}{K_{l}+1}} \widetilde{\boldsymbol{h}}_{\mathrm{dl}, l}^{\mathrm{H}} \text {. }
$$

The specular (LoS) and diffuse (scattered) components of the channel are denoted by $\overline{\boldsymbol{h}}_{\mathrm{dl}, l}^{\mathrm{H}}$ and $\widetilde{\boldsymbol{h}}_{\mathrm{dl}, l}^{\mathrm{H}}$, respectively. Note that $K_{l}$ is the unique Rice $(K)$ factor for the $l$-th terminal, denoting the ratio between the power of the specular and diffuse component in the propagation channel to terminal $l$ [14]. Moreover, $\widetilde{\boldsymbol{h}}_{\mathrm{dl}, l}^{\mathrm{H}} \sim \mathcal{C N}\left(0, \boldsymbol{I}_{M}\right)$, while the specular component of the channel is governed by the response of the ULA's transmit array steering vector [49]

$$
\overline{\boldsymbol{h}}_{\mathrm{d} 1, l}^{\mathrm{H}}=\left[1, e^{j 2 \pi d_{\mathrm{s}} \cos \left(\bar{\phi}_{l}\right)}, \ldots, e^{j 2 \pi d_{\mathrm{s}}(M-1) \cos \left(\bar{\phi}_{l}\right)}\right]
$$

Here, $d_{\mathrm{s}}$ is the equidistant antenna spacing normalized by the carrier wavelength and $\bar{\phi}_{l}$ is the direction-of-departure (DoD) of the specular component, for the l-th terminal. As uncorrelated downlink transmission is considered, the interelement spacing is set to a half-wavelength. Moreover, it is assumed that the $\bar{\phi}_{l}$ 's are uniformly distributed within the interval $[0,2 \pi)$. From the definition of the per-terminal channel in (5.1), a composite $L \times M$ small-scale fading channel matrix can be defined. This is given by $\boldsymbol{H}_{\mathrm{dl}}^{\mathrm{H}}=\left[\boldsymbol{h}_{\mathrm{dl}, 1}, \ldots, \boldsymbol{h}_{\mathrm{dl}, L}\right]^{\mathrm{H}}$. This can also 
be written as

$$
\boldsymbol{H}_{\mathrm{dl}}^{\mathrm{H}}=\Psi^{\frac{1}{2}}\left(\sqrt{\bar{K}_{\mathrm{av}}} \overline{\boldsymbol{H}}_{\mathrm{dl}}^{\mathrm{H}}+\widetilde{\boldsymbol{H}}_{\mathrm{dl}}^{\mathrm{H}}\right),
$$

where $\bar{K}_{\mathrm{av}}=(1 / L) \sum_{l=1}^{L} K_{l}, \boldsymbol{\Psi}=\operatorname{diag}\left(\frac{1}{K_{1}+1}, \ldots, \frac{1}{K_{L}+1}\right)$, the $L \times M$ composite specular channel matrix is denoted by $\overline{\boldsymbol{H}}_{\mathrm{dl}}^{\mathrm{H}}=\left[\sqrt{\frac{K_{1}}{\bar{K}_{\mathrm{av}}}} \overline{\boldsymbol{h}}_{\mathrm{dl}, 1}^{\mathrm{H}}, \ldots, \sqrt{\frac{K_{L}}{\bar{K}_{\mathrm{av}}}} \overline{\boldsymbol{h}}_{\mathrm{dl}, L}^{\mathrm{H}}\right]$ and $\widetilde{\boldsymbol{H}}_{\mathrm{dl}}^{\mathrm{H}}$ is the $L \times M$ composite diffuse channel matrix, respectively. The distribution of terminals in the cell is modeled as a uniform random variable with respect to the cell area. Furthermore, the link gain specific to terminal $l$ is given by

$$
\tilde{\beta}_{l}=v\left(\frac{d_{0}}{d_{l}}\right)^{\alpha} \zeta_{l}
$$

and is composed of the large-scale fading effects. ${ }^{2}$ In particular, $v$ is the unit-less constant for the geometric attenuation at a reference distance $d_{0}, d_{l}$ is the link distance between the BS and the $l$-th terminal, $\alpha$ is the attenuation exponent and $\zeta_{l}$ represents the effects of shadow fading which follows a log-normal distribution, i.e., $10 \log _{10}\left(\zeta_{l}\right) \sim \mathcal{N}\left(0, \sigma_{\text {sf }}^{2}\right)$. Since $\Psi^{\frac{1}{2}}$ simply scales the individual terminal channels, the overall channel can be defined as $\boldsymbol{G}_{\mathrm{dl}}^{\mathrm{H}}=\boldsymbol{D}^{\frac{1}{2}} \boldsymbol{H}_{\mathrm{dl}}^{\mathrm{H}}$, where $\boldsymbol{D}=$ $\operatorname{diag}\left(\beta_{1}, \ldots, \beta_{L}\right)$, is the $L \times L$ diagonal matrix of equivalent link gains, in which

$$
\beta_{l}=[\Psi]_{l, l} \tilde{\beta}_{l}=\tilde{\beta}_{l}\left(\frac{1}{K_{l}+1}\right)
$$

The above definition of the channel is used for the remainder of the chapter, which allows one to leverage previous analytical results on Ricean fading channels.

Conditioned on the cell size and the relative proximity of the terminals to the BS, a statistical model is employed, following $[82,84,136,137]$ to determine if a given terminal experiences LoS or NLoS propagation conditions. Naturally, the LoS and NLoS probabilities are a function of the link distance, from which the LoS and NLoS geometric attenuation and other link characteristics are obtained. As channel parameters from both the microwave and mmWave

\footnotetext{
${ }^{2}$ The link gain definition in (5.4) is consistent with the definitions in Chapters $3,4,6,7$ and 8 , respectively.
} 
frequency bands are to be considered, the propagation parameters are obtained from [136] for the former and [137] for the latter. For ease of notation and consistency, further discussion in relation to the above mentioned parameters is delayed to Section 5.5.

\subsubsection{RZF SINR and Ergodic Sum Spectral Efficiency}

Narrowband transmission is assumed with equal power allocation to each terminal. Perfect channel state information at the BS array is also assumed, in which case, the received signal at the $l$-th terminal can be written $\operatorname{as}^{3}$

$$
r_{\mathrm{dl}, l}=\rho_{\mathrm{dl}}^{\frac{1}{2}}\left(\frac{\beta_{l}}{\eta}\right)^{\frac{1}{2}} \boldsymbol{h}_{\mathrm{dl}, l}^{\mathrm{H}} \boldsymbol{w}_{l} s_{\mathrm{dl}, l}+\rho_{\mathrm{dl}}^{\frac{1}{2}}\left(\frac{\beta_{l}}{\eta}\right)^{\frac{1}{2}} \sum_{\substack{k=1 \\ k \neq l}}^{L} \boldsymbol{h}_{\mathrm{dl}, l}^{\mathrm{H}} \boldsymbol{w}_{k} s_{\mathrm{dl}, k}+n_{\mathrm{dl}, l} .
$$

Here, $\rho_{\mathrm{dl}}$ is the average downlink transmit power at the BS array, $\boldsymbol{w}_{l}$ is the unnormalized precoding vector from the BS to terminal $l$, and $s_{\mathrm{dl}, l}$ is the normalized data symbol desired for terminal $l$, such that $\mathbb{E}\left[\left|s_{l}\right|^{2}\right]=1$. Moreover, $\eta$ is the precoder normalization to ensure that the overall transmit power remains unchanged and $n_{\mathrm{dl}, l} \sim \mathcal{C N}\left(0, \sigma_{\mathrm{dl}, l}^{2}\right)$ models the effects of additive white Gaussian noise at $l$-th terminal. Throughout the remainder the chapter, it is assumed that $\sigma_{\mathrm{dl}}^{2}=\sigma_{\mathrm{dl}, 1}^{2}=, \ldots,=\sigma_{\mathrm{dl}, L}^{2}$. Thus, the link SNR, for an arbitrary link is defined as the ratio between the average downlink transmit power to the noise power at any given terminal, i.e., $\rho_{\mathrm{dl}} / \sigma_{\mathrm{dl}}^{2}$.

In this chapter, RZF precoding is considered to design the downlink precoding vectors, where $\boldsymbol{w}_{l}$ is the $l$-th column of the $M \times L$ precoding matrix, $\boldsymbol{W}$, defined as

$$
\boldsymbol{W}=\boldsymbol{H}_{\mathrm{dl}}\left(\boldsymbol{H}_{\mathrm{dl}}^{\mathrm{H}} \boldsymbol{H}_{\mathrm{dl}}+\xi \boldsymbol{I}_{L}\right)^{-1}
$$

Here, $\xi=L / \mathrm{SNR} \geq 0$ denotes the regularization parameter chosen from $[80,118]$ to maximize SINR at the terminal, where SNR refers to the link SNR. Following $[43,80,122]$, the RZF precoding matrix is normalized with $\eta=\|\boldsymbol{W}\|_{\mathrm{F}}^{2} / L$,

\footnotetext{
${ }^{3}$ The downlink system expressions are presented here for the ease of exposition and are consistent with the expressions in Chapters 3,4,6,7 and 8, respectively.
} 
ensuring the total average transmit power remains $\rho_{\mathrm{dl}}$. The received signal in (5.6) can be translated into a received SINR for terminal $l$, and expressed as

$$
\operatorname{SINR}_{\mathrm{dl}, l}=\frac{\rho_{\mathrm{dl}} \frac{\beta_{l}}{\eta}\left|\boldsymbol{h}_{\mathrm{dl}, l}^{\mathrm{H}} \boldsymbol{w}_{l}\right|^{2}}{\sigma_{\mathrm{dl}, l}^{2}+\rho_{\mathrm{dl}} \frac{\beta_{l}}{\eta} \sum_{\substack{i=1 \\ i \neq l}}^{L}\left|\boldsymbol{h}_{\mathrm{d} l, l}^{\mathrm{H}} \boldsymbol{w}_{i}\right|^{2}} .
$$

To this end, the instantaneous downlink spectral efficiency (measurable in bits/sec/Hz) at the $l$-th terminal can be computed as $\mathrm{R}_{\mathrm{dl}, l}=\log _{2}\left(1+\mathrm{SINR}_{\mathrm{dl}, l}\right)$. As such, the ergodic sum spectral efficiency is given by

$$
\mathbb{E}\left[\mathrm{R}_{\mathrm{dl}, \mathrm{sum}}\right]=\mathbb{E}\left[\sum_{l=1}^{L} \log _{2}\left(1+\operatorname{SINR}_{\mathrm{dl}, l}\right)\right]
$$

where the expectation is taken over the small-scale fading. In the following section, tight analytical expressions are derived to approximate the expected RZF SINR and ergodic sum spectral efficiency of the system.

\subsection{Expected SINR and Ergodic Sum Spectral Efficiency Approximations}

As motivated in Section 3.4.3 of Chapter 3, via the first-order delta method approximation, the expected SINR at the $l$-th user terminal can be approximated as $[68,80,84,86,118,138]$

$$
\mathbb{E}\left[\operatorname{SINR}_{\mathrm{dl}, l}\right] \approx \frac{\rho_{\mathrm{dl}} \frac{\beta_{l}}{\tilde{\eta}} \mathbb{E}\left[\left|\boldsymbol{h}_{\mathrm{dl}, l}^{\mathrm{H}} \boldsymbol{w}_{l}\right|^{2}\right]}{\sigma_{\mathrm{dl}, l}^{2}+\rho_{\mathrm{dl}} \frac{\beta_{l}}{\tilde{\eta}} \sum_{\substack{k=1 \\ k \neq l}}^{L} \mathbb{E}\left[\left|\boldsymbol{h}_{\mathrm{dl}, l}^{\mathrm{H}} \boldsymbol{w}_{k}\right|^{2}\right]},
$$

where $\tilde{\eta}=\mathbb{E}[\eta]$. In the sequel, the expectations in (5.10) for the respective signal and interference powers are derived separately, leading to the following subsections. 


\subsubsection{Computation of Expected Signal Power}

The expected signal power at the $l$-th terminal is given by

$$
\delta_{l}=\rho_{\mathrm{dl}} \frac{\beta_{l}}{\tilde{\eta}} \mathbb{E}\left[\left|\boldsymbol{h}_{\mathrm{dl}, l}^{\mathrm{H}} \boldsymbol{w}_{l}\right|^{2}\right]
$$

Now, via an eigenvalue decomposition, one can denote $\boldsymbol{H}_{\mathrm{dl}}^{\mathrm{H}} \boldsymbol{H}_{\mathrm{dl}}=\boldsymbol{U} \boldsymbol{\Lambda} \boldsymbol{U}^{\mathrm{H}}$. Then, from [118], the expected value of the desired signal power for terminal $l$ can be written as a function of the eigenvalues of $\boldsymbol{H}_{\mathrm{dl}}^{\mathrm{H}} \boldsymbol{H}_{\mathrm{dl}}$, an uncorrelated, non-central complex Wishart matrix. This is given by

$$
\varrho_{l}=\mathbb{E}\left[\left|\boldsymbol{h}_{\mathrm{d} 1, l}^{\mathrm{H}} \boldsymbol{w}_{l}\right|^{2}\right]=\mathbb{E}\left[\left(\sum_{l=1}^{L} \frac{\lambda_{l}}{\lambda_{l}+\xi}\left|u_{i, l}\right|^{2}\right)^{2}\right]
$$

Here, $\lambda_{l}$ is the $l$-th eigenvalue corresponding to the $l$-th diagonal entry in $\Lambda$ and $u_{i, l}$ denotes the entry of $\boldsymbol{U}$ corresponding to row $i$ and column $l$, respectively. Further taking the expectation over the entries of $\boldsymbol{U}$ allows one to express (5.12) as $[118]$

$$
\varrho_{l}=\frac{1}{L(L+1)}\left\{\mathbb{E}\left[\left(\sum_{l=1}^{L} \frac{\lambda_{l}}{\lambda_{l}+\xi}\right)^{2}\right]+\mathbb{E}\left[\sum_{l=1}^{m}\left(\frac{\lambda_{l}}{\lambda_{l}+\xi}\right)^{2}\right]\right\}
$$

Remark 5.3.1. It is to be noted that (5.13) relies on an isotropic distribution for $\boldsymbol{U}$, which does not hold for a fixed specular component in the propagation channel. However, if averaged over many random DoD values in the ULA's steering response vector, $\boldsymbol{U}$ retains its isotropic properties leading to (5.13). The expectations in (5.13) can be evaluated further over the eigenvalue densities of $\boldsymbol{H}_{\mathrm{dl}}^{\mathrm{H}} \boldsymbol{H}_{\mathrm{dl}}$, and are presented in the subsequent theorems.

Theorem 5.3.1. Let $\mu>0$ and $\phi_{1}, \ldots, \phi_{L}$ be the $L$ eigenvalues of $\bar{K}_{\mathrm{av}} \overline{\boldsymbol{H}}_{\mathrm{dl}}^{\mathrm{H}} \overline{\boldsymbol{H}}_{\mathrm{H}}$, then the expected value of $\sum_{l=1}^{L} \frac{\left(\lambda_{l}\right)^{\mu}}{\left(\lambda_{l}+\xi\right)^{2}}$ w.r.t. the eigenvalues of $\boldsymbol{H}_{\mathrm{dl}}^{\mathrm{H}} \boldsymbol{H}_{\mathrm{dl}}$, an un- 
correlated non-central complex Wishart matrix, is given by

$$
\begin{aligned}
S_{l}^{\mu}= & L\left[\Theta \sum_{j=1}^{L} \sum_{\substack{i=1 \\
i \neq j}}^{L} \sum_{p=0}^{\infty} \frac{\left(\bar{K}_{\mathrm{av}}+1\right)^{M-L+j}\left(\left(\bar{K}_{\mathrm{av}}+1\right) \phi_{i}\right)^{p} \mathcal{D}(i, j)}{p !(M-L+1)_{p}} \sum_{\gamma=0}^{\bar{\mu}}\left(\begin{array}{l}
\bar{\mu} \\
\gamma
\end{array}\right)(-\xi)^{\bar{\mu}-\gamma}\right. \\
& \left.\times e^{\xi\left(\bar{K}_{\mathrm{av}}+1\right)} \int_{\xi}^{\infty} x^{\gamma-2} e^{-x\left(\bar{K}_{\mathrm{av}}+1\right)} d x\right]
\end{aligned}
$$

Here, $\bar{\mu}=\mu-1+M-L+j$,

$$
\Theta=\frac{e^{-\sum_{l} \phi_{l}}}{L((M-L) !)^{L} \prod_{k<q}^{m}\left(\phi_{q}-\phi_{k}\right)}
$$

and $\mathcal{D}(i, j)$ is the $(i, j)$-co-factor of the $L \times L$ matrix $\boldsymbol{A}$, whose $(q, k)$-th entry is given by $(\boldsymbol{A})_{q, k}=(M-L+k-1) !{ }_{1} F_{1}\left(M-L+k, M-L+1, \phi_{q}\right)$, with ${ }_{1} F_{1}$ being a Kummer confluent hypergeometric function [18]. Moreover, $(M-L+1)_{p}=$ $\frac{(M-L+p) !}{(M-L) !}$ and

$$
\int_{\xi}^{\infty} x^{\gamma-2} e^{-x\left(\bar{K}_{\mathrm{av}}+1\right)} d x=\frac{1}{\left(\bar{K}_{\mathrm{av}}+1\right)^{\gamma-1}} \begin{cases}-\operatorname{Ei}(1, \tilde{\xi})+\frac{\mathrm{e}^{-\tilde{\xi}}}{\tilde{\xi}^{2}} & ; \gamma=0 \\ \operatorname{Ei}(1, \tilde{\xi}) & ; \gamma=1 \\ \Gamma(\gamma-1, \tilde{\xi}) & ; \gamma \geq 2\end{cases}
$$

where $\tilde{\xi}=\xi\left(\bar{K}_{\mathrm{av}}+1\right), \operatorname{Ei}(\cdot, \cdot)$ is the generalized exponential integral and $\Gamma(\cdot, \cdot)$ is the incomplete gamma function, respectively [80,139].

Proof of Theorem 5.3.1. One can begin by recognizing

$$
S_{l}^{\mu}=\mathbb{E}_{\lambda}\left[\sum_{l=1}^{L} \frac{\left(\lambda_{l}\right)^{\mu}}{\left(\lambda_{l}+\xi\right)^{2}}\right]=L\left\{\left[\int_{0}^{\infty} \frac{\left(\lambda_{\mathrm{arb}}\right)^{\mu}}{\left(\lambda_{\mathrm{arb}}+\xi\right)^{2}} f_{0}\left(\lambda_{\mathrm{arb}}\right) d \lambda_{\mathrm{arb}}\right]\right\}
$$

where $f_{0}\left(\lambda_{\mathrm{arb}}\right)$ is the density of an arbitrary eigenvalue selected from $\lambda_{1}, \ldots, \lambda_{L}$. 
Using $f_{0}\left(\lambda_{\text {arb }}\right)$ defined in (2.52) of Chapter 2 allows one to write (5.17) as

$$
\begin{aligned}
& S_{l}^{\mu}=\mathbb{E}_{\lambda}\left[\sum_{l=1}^{L} \frac{\left(\lambda_{l}\right)^{\mu}}{\left(\lambda_{l}+\xi\right)^{2}}\right]=L\left\{\left[\int_{0}^{\infty} \frac{\left(\lambda_{\mathrm{arb}}\right)^{\mu}}{\left(\lambda_{\mathrm{arb}}+\xi\right)^{2}} \frac{e^{-\sum_{l} \phi_{l}}}{L((M-L) !)^{L}} \frac{e^{-\lambda_{\mathrm{arb}}\left(\bar{K}_{\mathrm{av}}+1\right)}}{\lambda_{\mathrm{arb}}}\right.\right. \\
& \left.\left.\times \sum_{j=1}^{L}\left(\left(\bar{K}_{\mathrm{av}}+1\right) \lambda_{\mathrm{arb}}\right)^{M-L+j} \sum_{\substack{i=1 \\
i \neq j}}^{L} \sum_{p=0}^{\infty} \frac{\left(\left(K_{\mathrm{av}}+1\right) \phi_{i} \lambda_{\mathrm{arb}}\right)^{p} \mathcal{D}(i, j)}{p !(M-L+1)_{p}} /\left(\prod_{k<q}^{L} \phi_{q}-\phi_{k}\right) d \lambda_{\mathrm{arb}}\right]\right\} .
\end{aligned}
$$

Extracting the constants and simplifying (5.18) yields

$$
\begin{aligned}
S_{l}^{\mu} & =\mathbb{E}_{\lambda}\left[\sum_{l=1}^{L} \frac{\left(\lambda_{l}\right)^{\mu}}{\left(\lambda_{l}+\xi\right)^{2}}\right] \\
& =L\left\{\left[\frac{e^{-\sum_{l} \phi_{l}}}{L((M-L) !)^{L} \prod_{k<q}^{L}\left(\phi_{q}-\phi_{k}\right)} \sum_{\substack { j=1 \\
\begin{subarray}{c}{i=1 \\
i \neq j{ j = 1 \\
\begin{subarray} { c } { i = 1 \\
i \neq j } } \end{subarray} \sum_{p=0}^{L}\left(\bar{K}_{\mathrm{av}}+1\right)^{M-L+j}}\right.\right. \\
& \left.\left.\times \frac{\left(\left(\bar{K}_{\mathrm{av}}+1\right) \phi_{i}\right)^{p} \mathcal{D}(i, j)}{p !(M-L+1)_{p}} \int_{0}^{\infty} \frac{\lambda_{\mathrm{arb}}^{\mu-1+M-L+j+p} e^{-\lambda_{\mathrm{arb}}\left(\bar{K}_{\mathrm{av}}+1\right)}}{\left(\lambda_{\mathrm{arb}}+\xi\right)^{2}} d \lambda_{\mathrm{arb}}\right]\right\} .
\end{aligned}
$$

Letting $\bar{\mu}=\mu-1+M-L+j+p \geq 2$, the integral in (5.19) can be evaluated via a change of variables, such that $\lambda_{\text {arb }}=x-\xi$. This gives the integral in (5.19) as

$$
\sum_{\gamma=0}^{\bar{\mu}}\left(\begin{array}{l}
\bar{\mu} \\
\gamma
\end{array}\right)(-\xi)^{\bar{\mu}-\gamma} e^{\xi\left(\bar{K}_{\mathrm{av}}+1\right)} \int_{\xi}^{\infty} x^{\gamma-2} e^{-x\left(\bar{K}_{\mathrm{av}}+1\right)} d x
$$

where the integral in (5.20) is as defined in (5.16). Substituting (5.20) into (5.19) and letting $\Theta=\frac{e^{-\sum_{l} \phi_{l}}}{L((M-L) !)^{L} \prod_{k<q}^{L}\left(\phi_{q}-\phi_{k}\right)}$ yields the desired expression in (5.14).

Theorem 5.3.2. With $\phi_{1}, \ldots, \phi_{L}$ as the $L$ eigenvalues of $\bar{K}_{\mathrm{av}} \overline{\boldsymbol{H}}_{\mathrm{dl}}^{\mathrm{H}} \overline{\boldsymbol{H}}_{\mathrm{dl}}$, the expected value of $\left(\sum_{l=1}^{L} \frac{\lambda_{l}}{\lambda_{l}+\xi}\right)^{2}$ w.r.t. the eigenvalues of $\boldsymbol{H}_{\mathrm{dl}}^{\mathrm{H}} \boldsymbol{H}_{\mathrm{dl}}$ is given by

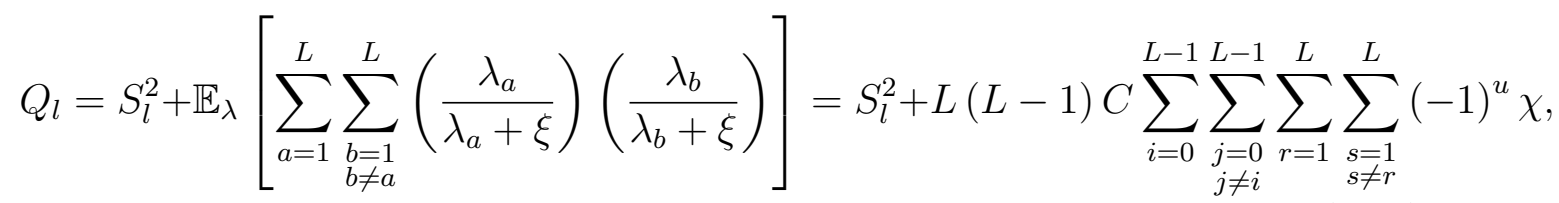

where $C=\Theta[(M-L) !]^{L}(-1)\left\lfloor\frac{L}{2}\right\rfloor(M-2) !$ and $u=i+j+r+s-p(i, j)-t(r, s)$ 
with

$$
p(i, j)=\left\{\begin{array}{l}
0 ; j \leq i \\
1 ; j>i
\end{array} \text { and } t(r, s)=\left\{\begin{array}{l}
0 ; s \leq r \\
1 ; s>r .
\end{array}\right.\right.
$$

Furthermore,

$$
\begin{aligned}
\chi & =\widetilde{\Xi}(r, s ; i, j) J_{r, i} J_{s, j} \\
& =\left(\phi_{r} \phi_{s}\right)^{-\frac{M-L}{2}} \boldsymbol{\Xi}(r, s ; i, j),
\end{aligned}
$$

where $\boldsymbol{\Xi}(r, s ; i, j)$ is a determinant with rows $r, s$ and columns $i, j$ removed, where the $d$-th entry of the $f$-th column is given by $\frac{\Gamma(M-L+f)}{\Gamma(M-L+1)}{ }_{1} F_{1}\left(M-L+f, M-L+1, \phi_{d}\right)$. Moreover,

$$
J_{a, b}=\sum_{\epsilon=0}^{\infty}\left[\frac{\phi_{a}^{\epsilon+\frac{M-L}{2}}}{\epsilon !(\epsilon+M-L) !}\right] \sum_{\gamma=0}^{\hat{\mu}}\left(\begin{array}{l}
\hat{\mu} \\
\gamma
\end{array}\right)(-\xi)^{\hat{\mu}-\gamma} e^{\xi} \int_{\xi}^{\infty} x^{\gamma-1} e^{-x} d x
$$

where $\hat{\mu}=\epsilon+M-L+b+1 \geq 2$ and the integral in (5.24) is a special case of the integral defined in (5.16).

Proof of Theorem 5.3.2. Using the joint density of a pair of arbitrary eigenvalues, say $\lambda_{1}$ and $\lambda_{2}$, derived in equation (2.54) of Chapter 2, one can begin by stating

$$
\begin{aligned}
Q_{l}= & S_{l}^{2}+L(L-1) \int_{0}^{\infty} \int_{0}^{\infty}\left(\frac{\lambda_{1}}{\lambda_{1}+\xi}\right)\left(\frac{\lambda_{2}}{\lambda_{2}+\xi}\right) C \sum_{i=0}^{L-1} \sum_{\substack{j=0 \\
j \neq i}}^{L-1} \sum_{r=1}^{L} \sum_{\substack{s=1 \\
s \neq r}}^{L}(-1)^{u} \tilde{\boldsymbol{\Xi}}(r, s ; i, j) \\
& \times g_{r, i}\left(\lambda_{1}\right) g_{s, j}\left(\lambda_{2}\right) d \lambda_{2} d \lambda_{1} .
\end{aligned}
$$

Recognizing that

$$
g_{a, b}(\lambda)=\lambda^{(M-L) / 2+b} e^{-\lambda} \mathrm{I}_{M-L}\left(2 \sqrt{\phi_{a} \lambda}\right)
$$

where $\mathrm{I}_{M-L}(\cdot)$ is the modified Bessel function of the first kind [63] and substi- 
tuting their respective definitions into (5.25) yields

$$
Q_{l}=S_{l}^{2}+L(L-1) \sum_{i=0}^{L-1} \sum_{\substack{j=0 \\ j \neq i}}^{L-1} \sum_{r=1}^{L} \sum_{\substack{s=1 \\ s \neq r}}^{L}(-1)^{u} \tilde{\Xi}(r, s ; i, j) J_{r, i} J_{s, j}
$$

for any two arbitrary eigenvalues $\left(\lambda_{1}, \lambda_{2}\right)$, where

$$
J_{a, b}=\int_{0}^{\infty} \frac{\lambda^{((M-L) / 2)+b+1} e^{-\lambda} \mathrm{I}_{M-L}\left(2 \sqrt{\phi_{a} \lambda}\right)}{\lambda+\xi} d \lambda,
$$

To evaluate the above integral, $\mathrm{I}_{M-L}\left(2 \sqrt{\phi_{a} \lambda}\right)$ has to be converted into its equivalent series form giving

$$
J_{a, b}=\sum_{\varepsilon=0}^{\infty}\left[\frac{\phi_{a}^{\varepsilon+\frac{M-L}{2}}}{\varepsilon !(\varepsilon+M-L) !}\right] \int_{0}^{\infty} \frac{\lambda^{\varepsilon+M-L+b+1} e^{-\lambda}}{\lambda+\xi} d \lambda .
$$

Denoting $\hat{\mu}=\varepsilon+M-L+b+1 \geq 2$, the integral in (5.29) can be evaluated with a change of variable, where $\lambda=x-\xi$. Upon doing this, after some algebraic simplifications, one can obtain

$$
\int_{0}^{\infty} \frac{\lambda^{\hat{\mu}} e^{-\lambda}}{\lambda+\xi} \lambda=\sum_{\gamma=0}^{\hat{\mu}}\left(\begin{array}{l}
\hat{\mu} \\
\gamma
\end{array}\right)(-\xi)^{\hat{\mu}-\gamma} e^{\xi} \int_{\xi}^{\infty} x^{\gamma-1} e^{-x} d x
$$

Note that the integral in (5.30) is a special case of the integral in (5.16). Substituting (5.30) into (5.29) and (5.29) into (5.27) yields the expression in Theorem 5.3 .2 .

Using the derived results in Theorem 5.3.1 and Theorem 5.3.2, the expression from (5.13) can be re-written as

$$
\varrho_{l}=\frac{Q_{l}+S_{l}^{2}}{L(L+1)}
$$

Therefore, the expected signal power for terminal $l$ from (5.11) can be written as

$$
\delta_{l}=\rho_{\mathrm{dl}} \frac{\beta_{l}}{\tilde{\eta}} \varrho_{l}
$$


The expected value of the precoder normalization parameter can also be extracted from the derived results such that

$$
\tilde{\eta}=\frac{1}{L}\left\{\mathbb{E}\left[\|\boldsymbol{W}\|_{\mathrm{F}}^{2}\right]\right\}=\frac{1}{L}\left\{\mathbb{E}_{\lambda}\left[\sum_{l=1}^{L} \frac{\lambda_{l}}{\left(\lambda_{l}+\xi\right)^{2}}\right]\right\}=\frac{1}{L} S_{l}^{1}
$$

\subsubsection{Computation of Expected Interference Power}

The total expected received power (desired and interfering powers) at the $l$-th terminal can be written as [118]

$$
\bar{\gamma}_{l}=\frac{1}{L}\left\{\mathbb{E}\left[\left\|\boldsymbol{H}_{\mathrm{dl}}^{\mathrm{H}} \boldsymbol{W}\right\|_{\mathrm{F}}^{2}\right]\right\}=\frac{1}{L}\left\{\mathbb{E}_{\lambda}\left[\sum_{l=1}^{m}\left(\frac{\lambda_{l}}{\lambda_{l}+\xi}\right)^{2}\right]\right\}=\frac{1}{L} S_{l}^{2} .
$$

Following $[80,118,139]$, the expected interference power at terminal $l$ is denoted by $\iota_{l}$, as the difference between the total expected received power and the expected signal power. The reason for computing the expected interference power was motivated in Remark 4.3.2 in Chapter 4. Therefore,

$$
\iota_{l}=\bar{\gamma}_{l}-\delta_{l}=\frac{S_{l}^{2}}{L}-\frac{Q_{l}+S_{l}^{(2)}}{L(L+1)}
$$

Using (5.31), (5.32) and (5.35), one can now write the expected SINR for the $l$-th terminal as a function of $\delta_{l}$ and $\tilde{\eta}$ and $\iota_{l}$ as

$$
\mathbb{E}\left[\mathrm{SINR}_{\mathrm{dl}, l}\right] \approx \frac{\rho_{\mathrm{dl}} \frac{\beta_{l}}{\tilde{\eta}} \delta_{l}}{\sigma_{\mathrm{d} l, l}^{2}+\rho_{\mathrm{dl}} \frac{\beta_{l}}{\tilde{\eta}}(L-1) \iota_{l}} .
$$

Remark 5.3.2. The generality of the results derived in Theorems 5.3.1 and 5.3.2 is worth mentioning. The theorems are applicable for any system dimension, link SNRs and hold for arbitrary rank LoS and NLoS channels [80]. The derived results can also be applied to other systems, such as small-cellular networks, where a hierarchy of BSs may be present. In such cases, with networkwide coordination, the additional presence of inter-cellular interference can be characterized in exactly the same manner as above [80].

The expected per-terminal SINR for terminal $l$ can be used to obtain the 
ergodic sum spectral efficiency over all $L$ terminals. This can now be approximated by

$$
\mathbb{E}\left[\mathrm{R}_{\mathrm{dl}, \mathrm{sum}}\right] \approx \sum_{l=1}^{L} \log _{2}\left(1+\mathbb{E}\left[\mathrm{SINR}_{\mathrm{dl}, 1]}\right]\right) .
$$

The accuracy of the expected per-terminal SINR and ergodic sum spectral efficiency approximations is demonstrated in Section 5.5 of the chapter. In what follows, at high link SNRs, a distributional approximation to the instantaneous RZF SINR is presented using ZF precoding.

\subsection{Approximation to the RZF SINR}

As mentioned earlier in the thesis in Chapters 3 and 4, the performance of RZF precoding converges to ZF precoding, as the link SNR is increased [80,118]. This is due to the that the regularization constant, $\xi=L /($ link SNR $) \rightarrow 0$, as $($ link SNR) $\rightarrow \infty$. The RZF SINR for terminal $l$ remains as defined earlier in (5.8), however, as ZF precoding completely eliminates multiuser interference, the received signal only composes of the desired signal power and additive white Gaussian noise. As a result, the received signal at terminal $l$ with ZF processing is given by

$$
r_{\mathrm{dl}, l}=\rho_{\mathrm{dl}}^{\frac{1}{2}}\left(\frac{\beta_{l}}{\eta}\right)^{\frac{1}{2}} \boldsymbol{h}_{\mathrm{dl}, l}^{\mathrm{H}} \boldsymbol{w}_{l} s_{\mathrm{dl}, l}+n_{\mathrm{dl}, l},
$$

where each parameter in (5.38) is defined after (5.6). Now, as ZF precoding is employed to design the downlink precoding vectors to each terminal, $\boldsymbol{w}_{l}$ forms

the $l$-th column of the $M \times L$ precoding matrix, $\boldsymbol{W}=\boldsymbol{H}_{\mathrm{dl}}\left(\boldsymbol{H}_{\mathrm{dl}}^{\mathrm{H}} \boldsymbol{H}_{\mathrm{dl}}\right)^{-1}$. The ZF precoding matrix is normalized by $\eta=\|\boldsymbol{W}\|_{\mathrm{F}}^{2} / L$, ensuring that the total average transmit power remains $\rho_{\mathrm{dl}}$. From (5.38), the ZF SNR for terminal $l$ 
can be defined as $^{4}$

$$
\mathrm{SNR}_{\mathrm{dl}, l}^{\mathrm{ZF}}=\frac{\rho_{\mathrm{dl}} \beta_{l}}{\sigma_{\mathrm{dl} l, l}^{2} \eta}=\frac{\rho_{\mathrm{dl}} \beta_{l}}{\sigma_{\mathrm{dl}, l}^{2} \operatorname{tr}\left\{\left(\boldsymbol{H}_{\mathrm{dl}}^{\mathrm{H}} \boldsymbol{H}_{\mathrm{dl}}\right)^{-1}\right\}} .
$$

Remark 5.4.1. The distribution of the ZF SNR in (5.39) is particularly difficult to analyze, since it is a random function of the uncorrelated complex non-standard, non-central Wishart matrix formed by $\boldsymbol{H}_{\mathrm{dl}}^{\mathrm{H}} \boldsymbol{H}_{\mathrm{dl}}$. Hence, analytical expressions for the probability density and cumulative distribution appear intractable. Therefore, the distribution of the ZF SNR (and in turn the distribution of the RZF SINR at high link SNRs) is approximated with the gamma distribution. The motivation for considering the gamma distribution comes from previous studies which have shown that in the case when no LoS is present (i.e., in classical Rayleigh fading), the ZF SNR follows the chi-squared distribution [140], a special case of the gamma distribution. More general studies with minimum-mean-squared-error (MMSE) receive combining under spatially correlated Rayleigh fading [141] and the presence of LoS (where the desired terminal is subject to Ricean fading, whilst the multiuser interference is subject to Rayleigh fading [142]) have shown that the MMSE SINR is well approximated by the gamma distribution. Here, with ZF precoding, the above results are further extended by considering the most general scenario where each terminal (desired or interfering) experiences Ricean fading with a unique Rice factor. In order to make such an approximation, the shape and scale parameters of the gamma distribution have to be derived, as demonstrated in the subsequent proposition.

Proposition 5.4.1. If the downlink channel to terminal $l$ follows a Ricean distribution, as specified in (5.1) and $\mathrm{SNR}_{\mathrm{dl}, l}^{\mathrm{ZF}}$ is modeled as a gamma random variable, then $\vartheta=\operatorname{tr}\left\{\left(\boldsymbol{H}_{\mathrm{dl}}^{\mathrm{H}} \boldsymbol{H}_{\mathrm{dl}}\right)^{-1}\right\}$ follows an inverse gamma distribution, denoted by $\Gamma^{\mathrm{d}}(\omega, \nu)^{-1}$, with the shape and scale parameters

$$
\omega=2+\frac{\mathbb{E}[\vartheta]^{2}}{\operatorname{Var}[\vartheta]} \text { and } \nu=\frac{\rho_{\mathrm{dl}} \beta_{k}}{\left\{1+\frac{\mathbb{E}[\vartheta]^{2}}{\operatorname{Var}[\vartheta]}\right\} \mathbb{E}[\vartheta]}
$$

\footnotetext{
${ }^{4}$ Explicit definition of the ZF SNR is presented here for the ease of exposition. The definition is consistent with that in Chapters 3 and 4 , respectively.
} 
Method for Proposition 5.4.1. Note that $\omega$ and $\nu$ are derived via the method of moments. If $\vartheta^{-1}$ is $\Gamma^{\mathrm{d}}(\omega, \nu)$, using its standard properties one can observe that

$$
\mathbb{E}\left[\vartheta^{-1}\right]=((\omega-1) \nu)^{-1}
$$

and

$$
\operatorname{Var}\left[\vartheta^{-1}\right]=\left((\omega-1)(\omega-2) \nu^{2}\right)^{-1}
$$

Note that re-arranging (5.41) and (5.42) allows us to derive (5.40). Moreover, $\vartheta=\operatorname{tr}\left\{\left(\boldsymbol{H}_{\mathrm{dl}}^{\mathrm{H}} \boldsymbol{H}_{\mathrm{dl}}\right)^{-1}\right\}=\sum_{i=1}^{L} \lambda_{i}^{-1}$, where $\lambda_{i}$ is the $i$-th eigenvalue of $\boldsymbol{H}_{\mathrm{dl}}^{\mathrm{H}} \boldsymbol{H}_{\mathrm{dl}}$. From here, it is straightforward to show that $\mathbb{E}[\vartheta]=L \mathbb{E}\left[\lambda_{\text {arb }}^{-1}\right]$, where $\lambda_{\text {arb }}$ is an arbitrary eigenvalue of $\boldsymbol{H}_{\mathrm{dl}}^{\mathrm{H}} \boldsymbol{H}_{\mathrm{dl}}$. Also, as

$$
\operatorname{Var}[\vartheta]=\mathbb{E}\left[\sum_{i=1}^{L} \sum_{j=1}^{L}\left(\lambda_{i} \lambda_{j}\right)^{-1}\right]-L^{2} \mathbb{E}\left[\lambda_{\text {arb }}^{-1}\right]^{2}
$$

the first term of $\operatorname{Var}[\vartheta]$ can be re-written as $Y=\mathbb{E}\left[\sum_{i=1}^{L} \lambda_{i}^{-2}+\sum_{i=1}^{L} \sum_{j=1, j \neq i}^{L} \lambda_{i}^{-1} \lambda_{j}^{-1}\right]=$ $L \mathbb{E}\left[\lambda_{\text {arb }}^{-2}\right]+L(L-1) \mathbb{E}\left[\lambda_{1}^{-1} \lambda_{2}^{-1}\right]$, where $\lambda_{1}$ and $\lambda_{2}$ are two distinct arbitrary eigenvalues of $\boldsymbol{H}_{\mathrm{dl}}^{\mathrm{H}} \boldsymbol{H}_{\mathrm{dl}}$. Hence, $\mathbb{E}\left[\lambda_{\text {arb }}^{-1}\right], \mathbb{E}\left[\lambda_{\text {arb }}^{-2}\right]$ and $\mathbb{E}\left[\lambda_{1}^{-1} \lambda_{2}^{-1}\right]$ govern $\mathbb{E}[\vartheta]$ and $\operatorname{Var}[\vartheta]$, which are demonstrated in the subsequent analysis.

\subsubsection{Calculation of $\mathbb{E}\left[\lambda_{\text {arb }}^{-1}\right]$}

By definition

$$
\mathbb{E}\left[\lambda_{\mathrm{arb}}^{-1}\right]=\int_{0}^{\infty} \frac{1}{\lambda_{\mathrm{arb}}} f_{\lambda_{\mathrm{arb}}}\left(\lambda_{\mathrm{arb}}\right) d \lambda_{\mathrm{arb}}
$$

where $f_{\lambda_{\text {arb }}}\left(\lambda_{\text {arb }}\right)$ is the arbitrary eigenvalue density given in (2.52), utilized earlier in (5.17). From here, (5.44) can be expressed as

$$
\begin{aligned}
\mathbb{E}\left[\lambda_{\mathrm{arb}}^{-1}\right]= & \int_{0}^{\infty} \lambda_{\mathrm{arb}}^{-1} \frac{e^{-\sum_{i=1}^{L} \phi_{i}}}{L((M-L) !)^{L}} \frac{e^{-\lambda_{\mathrm{arb}}}\left(\bar{K}_{\mathrm{av}}+1\right)}{\lambda_{\mathrm{arb}}} \sum_{j=1}^{L}\left(\left(\bar{K}_{\mathrm{av}}+1\right) \lambda_{\mathrm{arb}}\right)^{a} \times \\
& \sum_{\substack{i=1 \\
i \neq j}}^{L} \sum_{p=0}^{\infty} \frac{\left(\left(\bar{K}_{\mathrm{av}}+1\right) \phi_{i} \lambda_{\mathrm{arb}}\right)^{p} \mathcal{D}(i, j)}{p !(M-L+1)_{p}} /\left\{\prod_{k<q}^{L}\left(\phi_{q}-\phi_{k}\right)\right\} d \lambda_{\mathrm{arb}},
\end{aligned}
$$


where each term in (5.45) is defined after (5.3) and (5.14), respectively. Extracting the constants and simplifying (5.45) yields

$$
\begin{gathered}
\mathbb{E}\left[\lambda_{\mathrm{arb}}^{-1}\right]=\frac{e^{-\sum_{i=1}^{L} \phi_{i}}}{L((M-L) !)^{L}} \frac{1}{\prod_{k<q}^{L}\left(\phi_{q}-\phi_{k}\right)} \sum_{j=1}^{L} \sum_{i=1}^{L} \sum_{p=0}^{\infty}\left(\bar{K}_{\mathrm{av}}+1\right)^{a} \times \\
\frac{\left(\left(\bar{K}_{\mathrm{av}}+1\right) \phi_{i}\right)^{p} \mathcal{D}(i, j)}{p !(M-L+1)_{p}} \int_{0}^{\infty} \lambda_{\mathrm{arb}}^{M-L+j+p-2} e^{-\lambda_{\mathrm{arb}}\left(\bar{K}_{\mathrm{av}}+1\right)} d \lambda_{\mathrm{arb}} .
\end{gathered}
$$

Let $\mu=M-L+j+p-2$. It can be observed the integral in (5.46) can be solved in closed-form as

$$
\int_{0}^{\infty} \lambda_{\mathrm{arb}}^{\mu} e^{-\lambda_{\mathrm{arb}}\left(\bar{K}_{\mathrm{av}}+1\right)} d \lambda_{\mathrm{arb}}=\mu !\left(\bar{K}_{\mathrm{av}}+1\right)^{-\mu-1}
$$

This is consistent with (5.16) when $\gamma \geq 2$. Substituting the solution of the integral above into (5.46) and multiplying the resultant expression by $L$ yields an expression for $\mathbb{E}[\vartheta]$.

\subsubsection{Calculation of $\mathbb{E}\left[\lambda_{\text {arb }}^{-2}\right]$}

Following the methodology in the calculation of $\mathbb{E}\left[\lambda_{\mathrm{arb}}^{-1}\right], \mathbb{E}\left[\lambda_{\mathrm{arb}}^{-2}\right]$ has the exact same form as $\mathbb{E}\left[\lambda_{\text {arb }}^{-1}\right]$, where $\mu$ in (5.46) and (5.47) is replaced by $\tilde{\mu}=M-L+$ $j+p-3$.

\subsubsection{Calculation of $\mathbb{E}\left[\lambda_{1}^{-1} \lambda_{2}^{-1}\right]$}

In order to evaluate $Y$, the first term of $\operatorname{Var}[\vartheta]$, we require $\mathbb{E}\left[\lambda_{1}^{-1} \lambda_{2}^{-1}\right]$. By definition

$$
\mathbb{E}\left[\lambda_{1}^{-1} \lambda_{2}^{-1}\right]=\int_{0}^{\infty} \int_{0}^{\infty} \lambda_{1}^{-1} \lambda_{2}^{-1} f_{\lambda_{1}, \lambda_{2}}\left(\lambda_{1}, \lambda_{2}\right) d \lambda_{1} d \lambda_{2},
$$

where $f_{\lambda_{1}, \lambda_{2}}\left(\lambda_{1}, \lambda_{2}\right)$ is the joint density of two distinct arbitrary eigenvalues, $\left(\lambda_{1}, \lambda_{2}\right)$, a novel result presented in Chapter 2 of the thesis (see (2.54)). Hence, 
(5.48) can be written as

$$
\mathbb{E}\left[\lambda_{1}^{-1} \lambda_{2}^{-1}\right]=\int_{0}^{\infty} \int_{0}^{\infty} C \sum_{i=0}^{L-1} \sum_{j=0}^{L-1} \sum_{r=1}^{L} \sum_{s=1}^{L}(-1)^{u} \tilde{\Xi}(r, s ; i, j) g_{r, i}\left(\lambda_{1}\right) g_{s, j}\left(\lambda_{2}\right) d \lambda_{2} d \lambda_{1},
$$

where each term in (5.49) is defined after (5.21). Substituting the definition of $g_{r, i}\left(\lambda_{1}\right)$ and $g_{s, j}\left(\lambda_{2}\right)$ into (5.49), and performing some mathematical simplifications results in

$$
\mathbb{E}\left[\lambda^{-1} \lambda_{2}^{-1}\right]=C \sum_{i=0}^{L-1} \sum_{j=0}^{L-1} \sum_{r=1}^{L} \sum_{s=1}^{L}(-1)^{w} \tilde{\boldsymbol{\Xi}}(r, s ; i, j) J_{r, i}\left(\lambda_{1}\right) J_{s, j}\left(\lambda_{2}\right),
$$

where the solutions to $J_{r, j}$ and $J_{s, j}$ are presented in (5.29) and (5.30), respectively. From this, the first term of $\operatorname{Var}[\vartheta], Y$, can be expressed as

$$
\begin{aligned}
Y= & {\left[L\left\{\Theta \sum_{j=1}^{L} \sum_{i=1}^{L} \sum_{p=0}^{\infty} \frac{\left(\bar{K}_{\mathrm{av}}+1\right)^{a}\left(\left(\bar{K}_{\mathrm{av}}+1\right) \phi_{i}\right)^{p} \mathcal{D}(i, j)}{p !(M-L+1)_{p}} \tilde{\mu} !\left(\bar{K}_{\mathrm{av}}+1\right)^{-\tilde{\mu}-1}\right\}\right.} \\
& \left.+L(L-1)\left\{\mathbb{E}\left[\left(\lambda_{1} \lambda_{2}\right)^{-1}\right]\right\}\right] .
\end{aligned}
$$

Subtracting $L^{2} \mathbb{E}\left[\lambda_{\text {arb }}^{-1}\right]^{2}$ from (5.51) yields the expression for Var $[\vartheta]$, concluding the proof.

Remark 5.4.2. In the derivation of $\mathbb{E}[\vartheta]$ and $\operatorname{Var}[\vartheta]$, the eigenvalue densities, $f_{\lambda}(\lambda)$ and $f_{\lambda_{1}, \lambda_{2}}\left(\lambda_{1}, \lambda_{2}\right)$, of the instantaneous channel correlation matrix, $\boldsymbol{G}^{\mathrm{H}} \boldsymbol{G}$, were used, which has an uncorrelated complex non-central Wishart structure. This is in contrast to prior work where a further approximation is often introduced to approximate the non-central structure by its central counterpart via an adjustment of the covariance matrix (see e.g., $[67,68]$ ). Furthermore, the result derived in Proposition 5.4.1 also holds for any system dimension (number of service antennas at the BS and terminals in the system) and link SNR. The result also is robust to the level of LoS present in the system. In Section 5.5, it is shown to remain tight for Rayleigh fading channels, which exhibit no LoS effects. The analysis methodology can easily be extended to other system types, 
such as multicellular systems and distributed antenna arrays, due to the general structure of the ZF SNR in (5.39).

In what follows, the numerical accuracy of the approximations derived in Sections 5.3 and 5.4 is evaluated.

\subsection{Numerical Results and Discussion}

In this section, numerical results for a UMi scenario are presented with RZF and ZF precoding with the parameters specified in Table 5.1. Unless otherwise specified, parameters for the microwave and mmWave cases were selected from [136] and [137], respectively. For both cases, circular cell radius of $100 \mathrm{~m}$ is employed with a $10 \mathrm{~m}$ exclusion radius. ${ }^{5}$ Based on the link distance, $d_{l}$, a probabilistic approach is employed to determine whether a given user terminal experiences LoS or NLoS propagation conditions. For the microwave case, the probability of the l-th user terminal experiencing LoS is given by [136]

$$
P_{\mathrm{LoS}}\left(d_{l}\right)=\left(\min \left(18 / d_{l}, 1\right)\left(1-e^{-d_{l} / 36}\right)\right)+e^{-d_{l} / 36}
$$

Naturally, the probability of terminal $l$ experiencing NLoS is then determined by $P_{\mathrm{NLOS}}=1-P_{\mathrm{LoS}}$. Equivalently, for the mmWave case [137],

$$
P_{\mathrm{LoS}}\left(d_{l}\right)=\left(1-P_{\text {out }}\left(d_{l}\right)\right) e^{-\bar{\omega}_{\mathrm{LoS}} d_{l}},
$$

where $1 / \bar{\omega}_{\mathrm{LoS}}=67.1 \mathrm{~m}$ and $P_{\text {out }}$ is the outage probability, occurring when the attenuation in either the LoS or NLoS states is sufficiently large. For simplicity, in this chapter, $P_{\text {out }}=0$ for simplicity in determining the LoS and NLoS probabilities. Note that (5.53) is also applicable for performance evaluation at frequencies higher than $28 \mathrm{GHz}$, such as $73 \mathrm{GHz}$ as shown in [137]. Upon determining the link state of each terminal, the corresponding link parameters are selected to model the large-scale propagation effects of geometric attenuation and shadow fading, as specified in Table 5.1. Note that $\sigma_{\mathrm{dl}}^{2}=\sigma_{\mathrm{dl}, 1}^{2}=, \ldots, \sigma_{\mathrm{dl}, L}^{2}=1$ and

\footnotetext{
${ }^{5}$ Note that this is the reference distance, $d_{0}$, in (5.4).
} 


\begin{tabular}{ccc}
\hline \hline Parameter & \multicolumn{2}{c}{ Value } \\
\cline { 2 - 3 } & Microwave & mmWave \\
\hline Carrier frequency $[\mathrm{GHz}]$ & 2 & 28 \\
LoS unit-less attenuation constant $[v]$ & 28 & 61.4 \\
NLoS unit-less attenuation constant & 22.7 & 72 \\
LoS attenuation exponent $[\alpha]$ & 2.2 & 2 \\
NLoS attenuation exponent & 3.67 & 2.92 \\
LoS shadow fading standard deviation $\left[\sigma_{\text {sf }}\right]$ & 3 & 5.8 \\
NLoS shadow fading standard deviation & 4 & 8.7 \\
$K$-Factor mean $[\mathrm{dB}]$ & 9 & $12[129]$ \\
$K$-Factor standard deviation $[\mathrm{dB}]$ & 5 & $3[129]$ \\
\hline
\end{tabular}

Table 5.1: Parameters used for the generation of numerical results.

thus the link SNR, defined as $\rho_{\mathrm{dl}} / \sigma_{\mathrm{dl}}^{2}=\rho_{\mathrm{dl}}$, for the results presented in the section. In the presented numerical results, the link SNR is referred to as SNR. Following [136] and [129], a unique $K$-factor, $K_{l}$, is assigned to the $l$-th user terminal from a log-normal distribution with the mean and standard deviation specified in Table 5.1. This is refereed to as $K \sim \ln$ (mean, standard deviation). It is worth observing that the mean of $K$ increases, while its standard deviation decreases, as one transitions from microwave to mmWave frequency bands.

First, the accuracy of the proposed expected per-terminal RZF SINR in (5.36) is examined. Fig. 5.1 illustrates the expected SINR for a given terminal as a function of link SNR for a system with $M=10$ and $L=3$. In addition to the microwave and mmWave cases, the uncorrelated Rayleigh fading case is considered as a baseline for comparison purposes. Also for comparison purposes, the case where each terminal is assigned a fixed $K$-factor of $5 \mathrm{~dB}$ is considered. Two trends can be observed: First is the fact that increasing the mean of $K$ has an adverse effect on the expected SINR. This is due to the fact that an increase in the mean $K$ implies a stronger specular component in the channel, which reduces multipath diversity and in turn reduces the channel rank. Equivalently, this effect can be interpreted by an increase in the level of correlation in the channel, leading to lower usable spatial degrees of freedom. This leads to higher multiuser interference and in turn a lower SINR is observed at a given terminal. Secondly, 


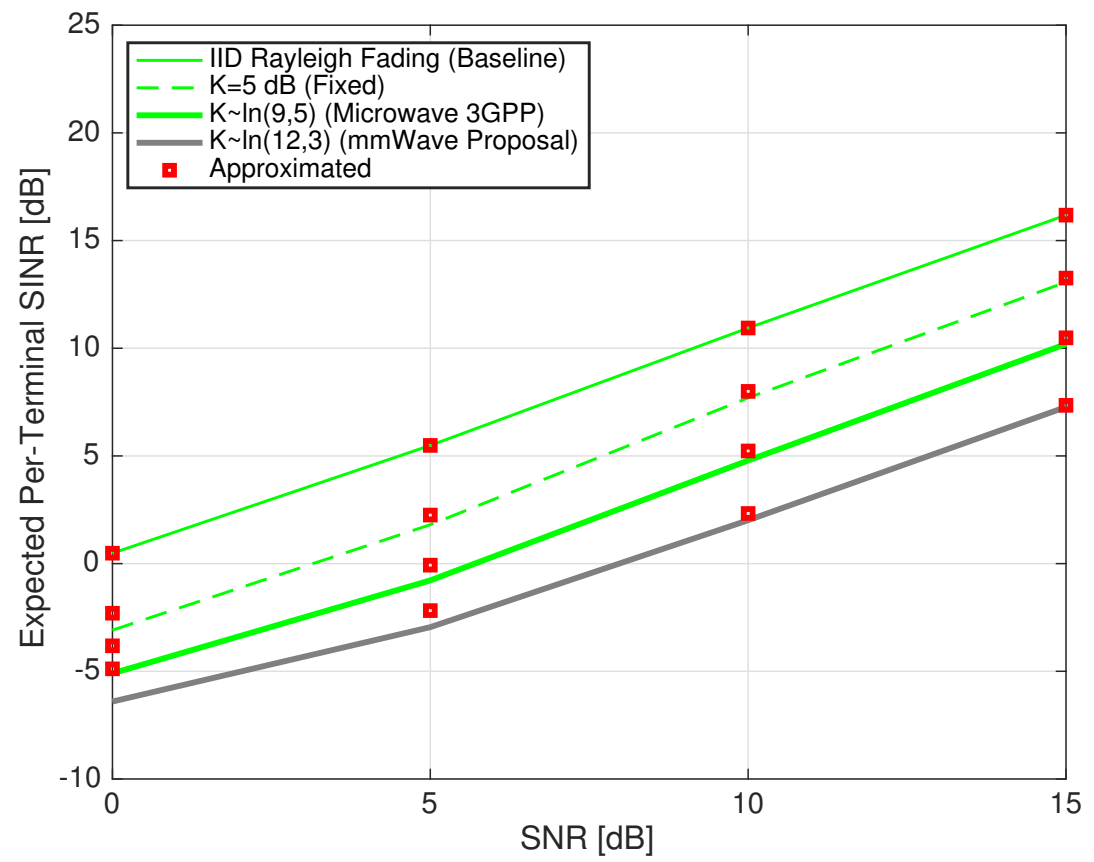

Figure 5.1: Expected per-terminal RZF SINR vs. link SNR with $M=10$ and $L=3$.

our proposed approximations are seen to remain sufficiently accurate for the entire link SNR range for all cases. It is worth mentioning that in most statistical channel models, the overall geometric attenuation (combined effect of distance based attenuation with shadow fading) for a given terminal is unrelated to its $K$ factor. In practice, LoS links are known to be associated with lower attenuation and multipath amplitude variation. In such cases, the resulting gains in the link budget may compensate for the loss of multipath diversity. Moreover, the results presented here are for the most commonly occurring scenarios, where the composite specular channel matrix may have a unit rank. It has been shown in [24] that for certain array geometries giving a full rank specular matrix, Ricean fading behaves like Rayleigh fading. Thus, it is likely that results for higher rank specular channels will be scenario dependent.

Now the impact of increasing $M$ on the expected per-terminal RZF SINR is studied with a fixed number of terminals in the system. Fig. 5.2 depicts the expected per-terminal SINR as a function of $M$ with $L=5$ at $\mathrm{SNR}=10$ dB. We observe that increasing $M$ naturally increases the expected per-user 


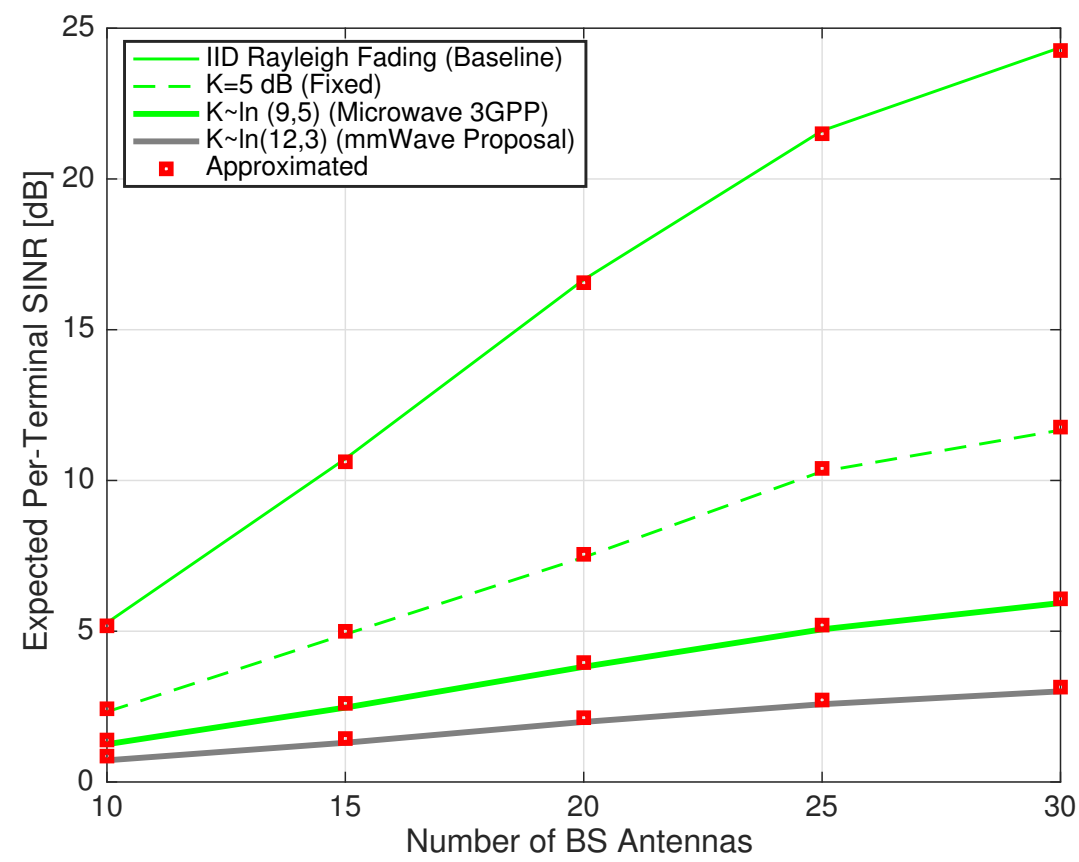

Figure 5.2: Expected per-terminal RZF SINR vs. $M$ with $L=5$ at link SNR $=$ $10 \mathrm{~dB}$.

SINR. However, the expected SINRs can be seen to slowly saturate with growing $M$. This is a result of channels to multiple terminals becoming asymptotically pairwise orthogonal, such that the inner product of any two channel vectors tends to zero. As mentioned earlier in the thesis, this effect has been famously coined as convergence to favorable propagation conditions in the large MUMIMO literature $[11,89]$. However, as uncorrelated downlink transmission is considered with a fixed inter-element spacing, the size of the ULA grows with increasing numbers of transmit antenna elements. Taking this into account, only up to $M=30$ transmit antennas are considered in the numerical result with an inter-element spacing of a half wavelength. It can also be observed that an increase in the mean of $K$ results in a slower growth in the expected per-terminal RZF SINR. For all cases, the derived approximations remain tight and are robust to changes in system size. This is consistent with Remark 5.3.2, presented earlier in the chapter.

Further to the above, the influence of LoS on the RZF ergodic sum spectral efficiency is now examined. Specifically, in Fig. 5.3, with $M=10$ and $L=3$ at 


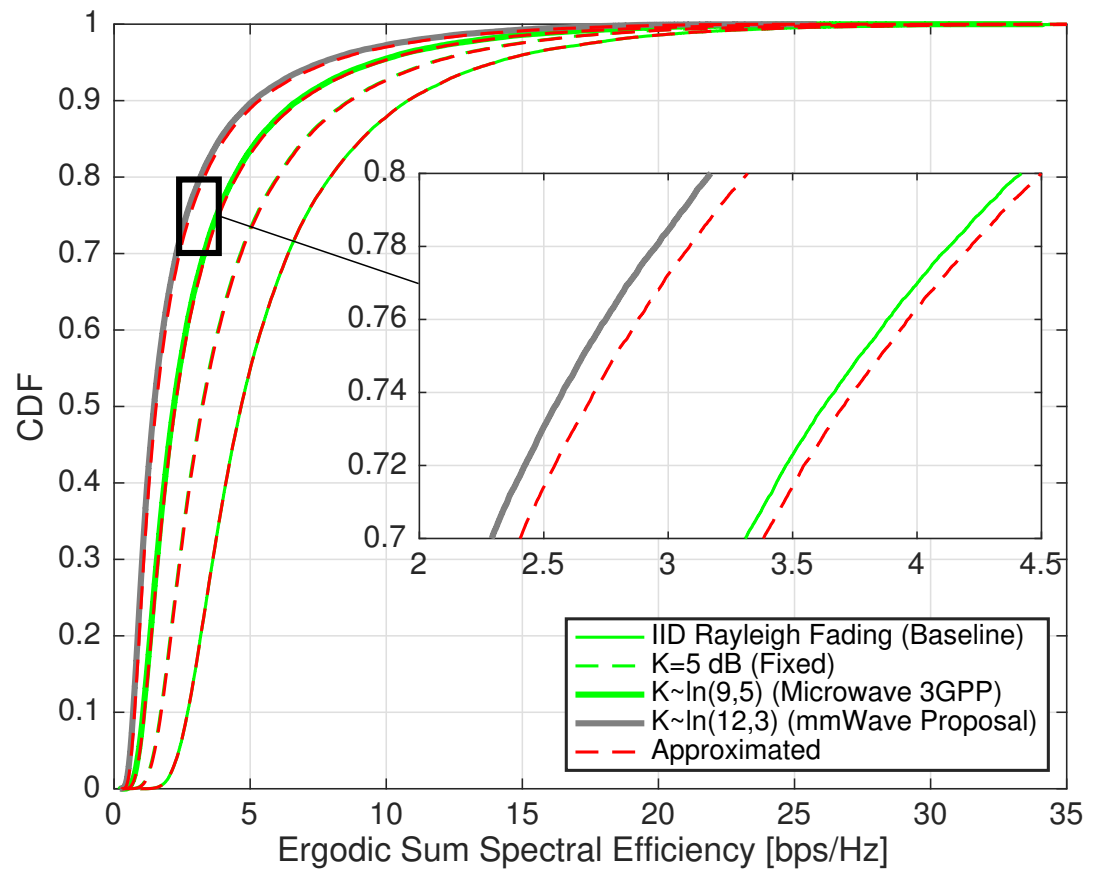

Figure 5.3: Ergodic sum-rate CDFs with $M=10, L=3$ at link SNR $=10 \mathrm{~dB}$.

link $\mathrm{SNR}=10 \mathrm{~dB}$, the cumulative distribution functions (CDFs) of the derived ergodic sum spectral efficiency approximation in (5.37) is compared with its simulated counterpart. The RZF ergodic sum spectral efficiency is obtained by averaging over fast-fading in the channel with the CDFs representing the variations in the link gains and the $K$ factors. It can be observed that although the approximations remain extremely tight for all considered cases, the ergodic sum spectral efficiencies achieved from (5.37) are marginally higher than the simulated responses. It is again observed that a stronger specular component has an adverse effect on the ergodic sum spectral efficiency, which is seen to degrade with increasing LoS powers.

As a further matter, an investigation is made into the impact of $K$-factor variability, for a fixed mean value, on the ergodic sum spectral efficiency. Fig. 5.4 depicts the RZF ergodic sum spectral efficiency at the CDF values of $0.1,0.5$ and 0.9 , respectively. It can again be observed that increasing the variability of $K$ at the CDF values of 0.1 and 0.5 leads to a degradation in the ergodic sum spectral efficiency. More interestingly, in contrast to this, at the upper end of 


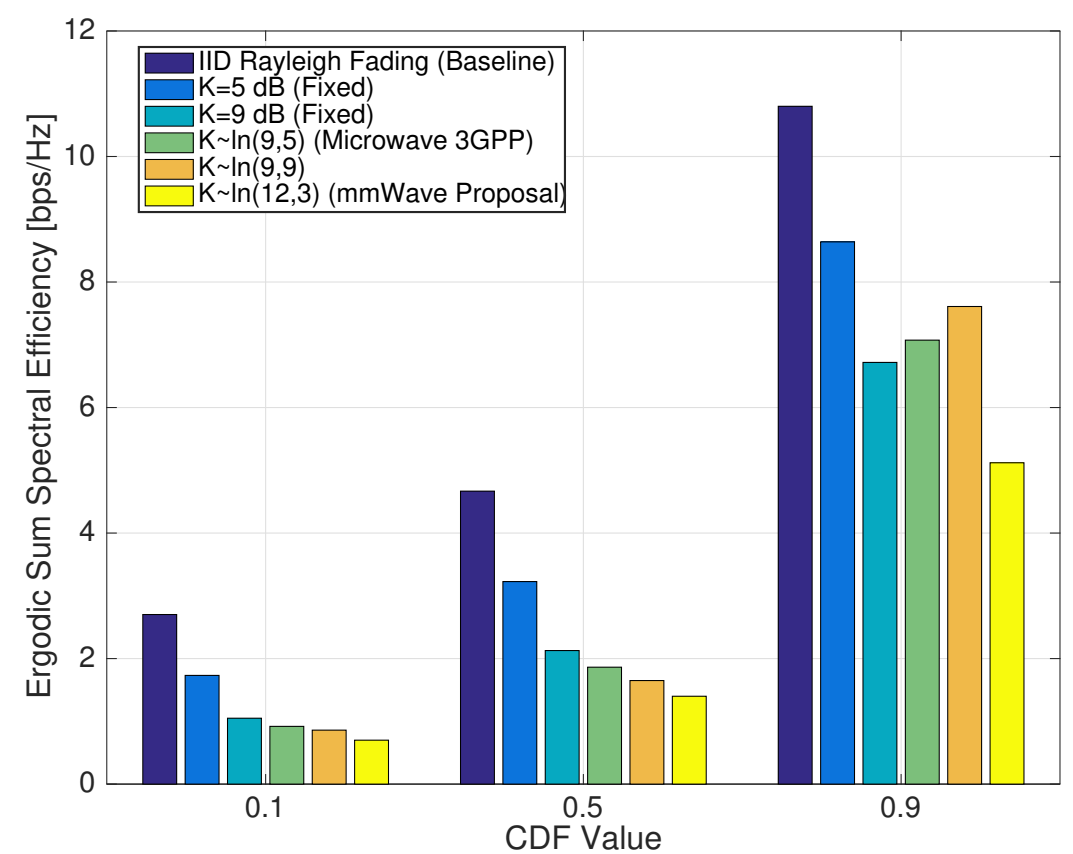

Figure 5.4: Ergodic sum spectral efficiency comparison at CDF values of 0.1, 0.5 and 0.9 with $M=10, L=3$ at link $\mathrm{SNR}=10 \mathrm{~dB}$.

the CDF (the peak ergodic sum spectral efficiency scenario with $\mathrm{CDF}=0.9$ ), increasing the variability of the $K$-factor improves the ergodic sum spectral efficiency. This behavior is related to the log-normal nature of the $K$-factor distribution. Increasing the standard deviation amplifies the range of $K$-factors from zero to very large values. Hence, at the lower end of the sum spectral efficiency $\mathrm{CDF}$, the increased occurrence of large $K$-factor values tends to reduce performance. This can be observed in Fig. 5.4 at CDF probabilities of 0.1 and 0.5. However, at the upper end of the CDF, where the ergodic sum spectral efficiency is already high, the increased variability of the $K$-factor helps, as more $K$-factor values close to zero are generated. Essentially, the increased variability in the $K$-factor increases the variability in the ergodic sum spectral efficiency. As a result, correspondingly the cell-edge spectral efficiencies are lowered, while the peak spectral efficiencies are enhanced.

Considering the same cases presented in the above figures, Fig. 5.5 evaluates the tightness of the gamma distributional approximation to the instantaneous RZF SINR, with ZF precoding at high link SNRs. With $M=30$ and $L=3$, 
for multiple link SNR levels, it can be observed that the ZF SNR tightly approximates the RZF SINR. ${ }^{6}$ Furthermore, it can be observed that the proposed gamma distributional approximations are shown to be extremely tight and are insensitive to changes in the link SNRs, where only the most marginal deviation is observed between the approximated and the simulated responses for all cases. This is consistent with Remark 5.4.2, earlier in the thesis. The impact of increasing the Rice factor variability is also investigated on the ZF SNR. In this regard, Fig. 5.6 evaluates the accuracy of the distributional approximation with $M=10$ and $M=60$ service antennas at the ULA, communicating with $L=3$ terminals at a link SNR of $10 \mathrm{~dB}$. Naturally, increasing the number of service antennas leads to higher terminal SNRs/SINRs. More critically though, increasing the variability of the $K$-factor from $K=9 \mathrm{~dB}$ fixed for all terminals to a variable $K$-factor with a mean of $9 \mathrm{~dB}$ and a standard deviation of $5 \mathrm{~dB}$ enhances the peak ZF SNR, for the same reason as in Fig. 5.4. The cell-edge and the median $\mathrm{ZF}$ can be seen to reduce, as the magnified $K$-factor variability also produces large $K$-factor values, leading to performance deterioration.

\subsection{Concluding Remarks}

The chapter investigated the impact of LoS on downlink MU-MIMO systems with linear precoding techniques. More specifically, the chapter presented tight analytical approximations to the expected per-terminal SINR and resulting ergodic sum spectral efficiency of a downlink MU-MIMO system with RZF precoding under Ricean fading channels. The analysis is robust to changes in system size, link SNR levels and can be applied to both LoS and NLoS channels. The density of an arbitrary eigenvalue and a joint density of a pair of arbitrary eigenvalues of the complex non-central Wishart matrix were shown to be fundamental to the analysis. With both the microwave and mmWave propagation parameters, the presented numerical results suggested that increasing

\footnotetext{
${ }^{6}$ For the subsequent figures, in order not to clutter the numerical results and information presented, only one example of the RZF SINR is demonstrated for the microwave case where $K \sim \ln (9,5)$.
} 


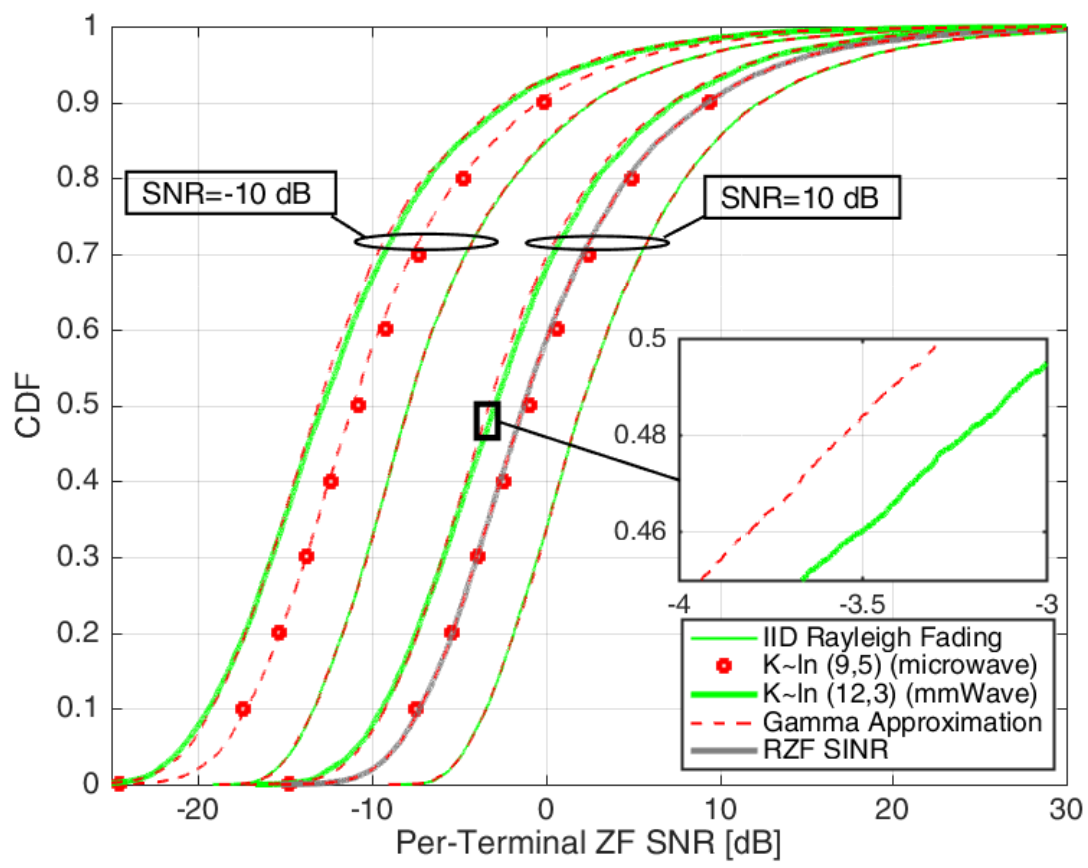

Figure 5.5: Per-terminal ZF SNR distribution with $M=30, L=3$ at link SNR levels of -10 and $10 \mathrm{~dB}$.

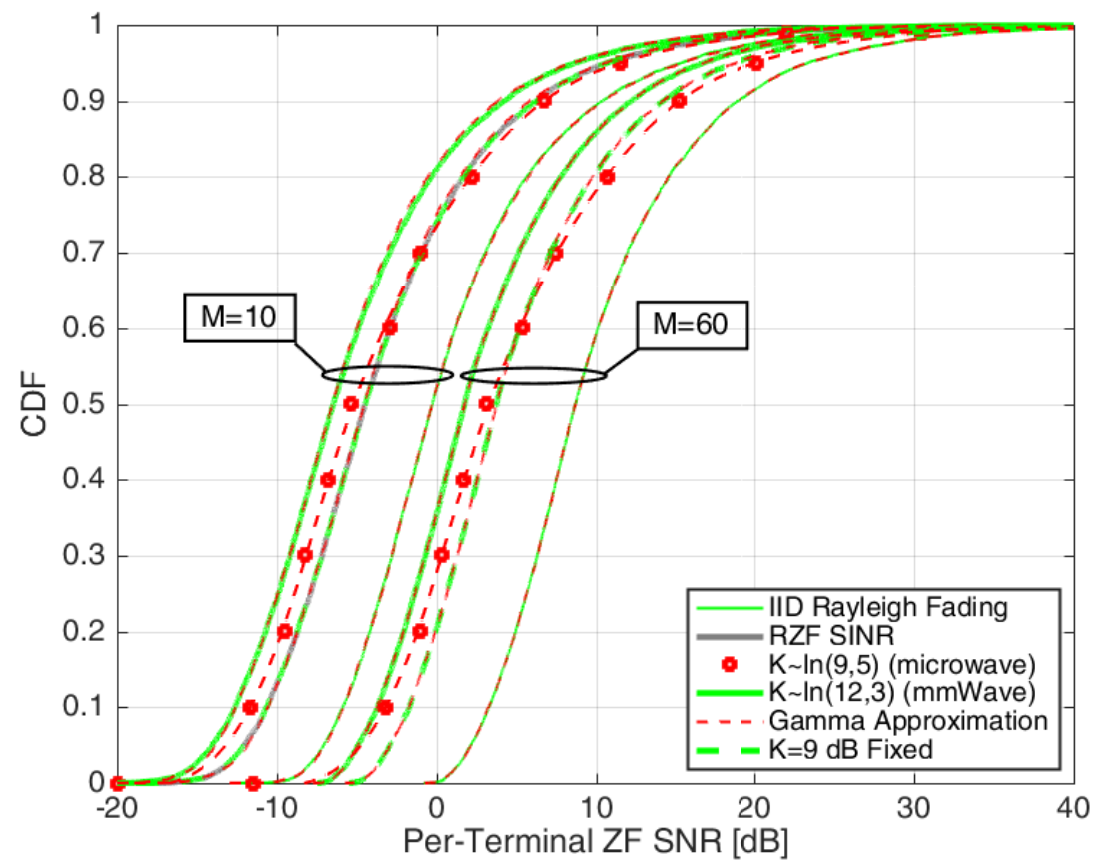

Figure 5.6: Per-terminal ZF SNR distribution with $M=10$ and 60 and $L=3$ at link $\mathrm{SNR}=10 \mathrm{~dB}$. 
the specular component of the propagation channel has an adverse effect on the expected SINR and ergodic sum spectral efficiency. Furthermore, increasing the variability of $K$-factor enhances the peak ergodic sum spectral efficiency, while reducing the median and cell-edge spectral efficiencies. To the best of the author's knowledge, the evaluation of the variability of $K$-factors is novel and can help to identify the sensitivities in multiuser system performance. The same conclusions held in the high SNR regime, where the instantaneous RZF SINR was approximated with a ZF SNR for a given terminal. It was shown that the ZF SNR approximately followed a gamma distribution, and the gamma parameters were derived. The distributional approximation was also robust to changes in system size, link SNR levels and arbitrary LoS levels. Such heterogeneity in the channel has not been handled well by previous studies. 


\section{Chapter 6}

\section{Simplified Performance Analysis With Linear Transceivers}

Summary. There is now a consensus amongst the cellular communications community that large antenna arrays will be required for handling the growing spectral efficiency demands of future cellular systems. While this is likely to push conventional multiuser multiple-input multiple-output (MU-MIMO) systems to an extreme, in the near future, to retain wide area coverage capability in cellular networks operating at microwave frequencies, the scenario with moderate numbers of service antennas (between 10 and 100) at a cellular base station is of considerable interest. It is therefore important to analyze the performance of MU-MIMO systems with moderate numbers of BS antennas. The analysis should be insightful, simple and adaptable to a wide range scenarios and MUMIMO signal processing techniques. Traditionally, with linear signal processing at the transmit and receive ends of the link, the focus has been on exact analysis of conventional MU-MIMO systems, which lead to complex solutions that do not scale well with growing numbers of service antennas and terminals. On the other hand, the freedom to assume favorable propagation conditions with large antenna arrays have led wireless researchers to discover the fundamental limits of key performance metrics such as the terminal signal-to-interference-plus-noiseratio (SINR) and system spectral efficiency. Motivated by this, under independent and identically distributed Rayleigh fading, in this chapter, a simpli- 
fied analysis methodology is developed for a general class of linear transceivers. More specifically, the instantaneous and expected (average) per-terminal SINR, signal-to-noise-ratio (SNR), as well as the instantaneous and ergodic sum spectral efficiency is analyzed with matched filter and zero-forcing (ZF) precoding on the downlink and maximum-ratio combining, ZF and minimum-mean-squarederror-combining on the uplink. The approximation methodology is based on the identification of specific components in the above mentioned performance metrics which tend to stabilize for moderate numbers of BS antennas, and replacing only these specific components by their expected values. In general, the resulting expressions allows for clear insights to be drawn into the factors which most contribute to the above mentioned performance metrics. Numerical evaluation of the approximations demonstrate they are extremely accurate and stable for moderate numbers of BS antennas. Moreover, the approximation methodology is robust to the type of fading environment assumed, and easily extended to suit other statistical channel models, such as spatially correlated Ricean fading.

\subsection{Introduction}

Most visions of the future involve a migration from conventional multiuser multiple-input multiple-output (MU-MIMO) (which is defined as $M \leq 10$, where $M$ is the number of serving antennas at the base station (BS) array) to large MU-MIMO (which is defined as $M \geq 100$ ), where the BS is able to serve tens of user terminals simultaneously in the same time-frequency resource $[9,11,57]$. However, in the middle ground, the scenario with moderate numbers of service antennas $(10<M<100)$ is also very important, both for the medium-term future and for retaining wide area coverage in the microwave frequency bands. Here the antenna array sizes required at the BS might preclude those for large MU-MIMO. As the wireless infrastructure changes to handle higher spectral and energy efficiency demands, it is timely to consider performance analysis approaches which are simple yet accurate, adaptable and are able to handle varied deployments, featuring moderate numbers of service antennas at a BS. This is 
the focus of the chapter.

With conventional MU-MIMO systems, the focus has largely been on exact analysis of key performance metrics, such as the per-terminal signal-tointerference-plus-noise-ratio (SINR) and system spectral efficiency in independent and identically distributed (i.i.d.) Rayleigh fading channels (see $[22,23,110$, 124, 143-145] and the references therein). However, such types of analyses often lead to complex, non-insightful results. Moreover, much of the analysis remains analytically intractable, especially for non i.i.d. Rayleigh fading channels. In addition to this, such analyses are often difficult to generalize to any number of transmit and receive antennas, due to numerical stability ${ }^{1}$ issues, limiting their applicability $[76,124]$. In stark contrast to this, for large MU-MIMO systems, in the limit of an indefinite number of BS antennas, deterministic equivalents of the SINR and sum spectral efficiency have helped simplify the analysis with linear processing at the transmitter and the receiver, using random matrix asymptotics [59-61].

Motivated by the above, in this chapter, novel simplified approximations to the instantaneous and expected per-terminal SINR, signal-to-noise-ratio (SNR), instantaneous sum spectral efficiency, as well as the ergodic sum spectral efficiency of MU-MIMO systems are derived, with moderate numbers of BS antennas serving a multiplicity of single-antenna terminals. More specifically, on the downlink, matched filter (MF) and zero-forcing ( $\mathrm{ZF})$ precoding are analyzed, while on the uplink, maximum-ratio combining (MRC), ZF and minimum-meansquared-error (MMSE) combining methods are analyzed. The analysis methodology is built on identifying components of the performance metrics which tend to be stable for moderate numbers of service antennas and replacing only these components by their expected values. Hence, limiting (large MU-MIMO) results are selectively applied, such that the complexity of an exact analysis is avoided, while the most important variation in the performance metrics is maintained.

Keeping this in mind, the specific contributions of the chapter are as follows:

\footnotetext{
${ }^{1}$ Note that here the term "numerical stability" is used to indicate numerical accuracy problems using straightforward programming in numerical software.
} 
1. Under i.i.d. Rayleigh fading and a unique link gain for each terminal, simplified approximations are derived to analyze the downlink and uplink performance of moderate MU-MIMO systems for the general class of linear transceivers, mentioned above. All derived approximations are shown to be numerically stable for moderate numbers of service antennas.

2. The derived approximations allow us to draw direct insights into the most influential system and propagation parameters contributing to the perterminal and system performance. For most uplink and downlink performance metrics, these are predominantly a function of the link SNR (defined in Section 6.2), the link gain of a particular terminal, the number of BS antennas and the total number of terminals present in the system.

3. To demonstrate the accuracy of the derived analysis, numerical results are presented with both cell-wide and instantaneous performance indicators. More specifically, in cell-wide performance, each trial corresponds to a different channel drop, in contrast to the instantaneous performance, where one channel drop is considered, and the variation is averaged with many realizations of the instantaneous fast-fading. Even though the analysis methodology is simple, it is shown to be easily adaptable to other types of statistical channel models, amplifying the generality of the approximation approach.

The remainder of the chapter is organized as follows: Section 6.2 presents the downlink and uplink MU-MIMO system models with the necessary performance metrics. Considering MF and ZF precoding, section 6.3 presents the instantaneous and expected SINR, SNR, as well as the instantaneous and ergodic sum spectral efficiency analysis for downlink MU-MIMO systems. Section 6.4 presents the equivalent uplink MU-MIMO performance analysis with MRC, ZF and MMSE detectors. Section 6.5 evaluates the tightness of the simplified approximations and demonstrates ways in which the analysis methodology can be modified to suit other types and fading channels. Finally, section 6.6 presents the concluding remarks of the chapter. 


\subsection{Downlink and Uplink System Models}

A single-cell MU-MIMO system is considered, where the BS is equipped with an array of $M$ omni-directional transmit antennas with an ideal radiation pattern and no mechanical downtilt. The BS array communicates with $L$ single-antenna user terminals (with $M \geq L$ ), in the same time-frequency resource. Transmission over i.i.d. Rayleigh fading channels is considered, where each terminal experiences a unique link gain, composing of the large-scale fading effects of geometric attenuation and shadow-fading. Assuming perfect CSI at the BS array with no uplink and downlink power control, the $1 \times M$ downlink channel vector to terminal $l$ from the BS array is given by

$$
\boldsymbol{g}_{\mathrm{dl}, l}^{\mathrm{H}}=\beta_{l}^{\frac{1}{2}} \boldsymbol{h}_{\mathrm{dl}, l}^{\mathrm{H}},
$$

where $\boldsymbol{h}_{\mathrm{dl}, l}^{\mathrm{H}} \sim \mathcal{C N}\left(\mathbf{0}, \boldsymbol{I}_{M}\right)$ is the fast-fading channel vector with zero mean and unit variance entries, and

$$
\beta_{l}=v\left(\frac{d_{0}}{d_{l}}\right)^{\alpha} \zeta_{l},
$$

is the link gain, composed of distance based geometric attenuation and lognormal shadow-fading. More specifically, $v$ is the unit-less constant for geometric attenuation at a given reference distance, $d_{0}$, assuming perfect far-field transmit antennas, $d_{l}$ is the link distance from the BS to the $l$-th terminal. Furthermore, $\alpha$ is the geometric attenuation exponent and $10 \log _{10}\left(\zeta_{l}\right) \sim \mathcal{N}\left(0, \sigma_{\text {sf }}^{2}\right)$ with $\sigma_{\text {sf }}$ denoting the shadow-fading standard deviation. From the definition in (6.1), the $L \times M$ composite channel matrix is denoted as $\boldsymbol{G}_{\mathrm{dl}}^{\mathrm{H}}=\boldsymbol{D}^{\frac{1}{2}} \boldsymbol{H}_{\mathrm{dl}}^{\mathrm{H}}$, where $\boldsymbol{G}_{\mathrm{dl}}^{\mathrm{H}}=\left[\boldsymbol{g}_{\mathrm{dl}, 1}^{\mathrm{H}}, \ldots, \boldsymbol{g}_{\mathrm{dl}, L}^{\mathrm{H}}\right]$ contains the individual channels to each terminal, $\boldsymbol{D}^{\frac{1}{2}}=\operatorname{diag}\left(\beta_{1}^{\frac{1}{2}}, \ldots, \beta_{L}^{\frac{1}{2}}\right)$ is the $L \times L$ diagonal matrix of link gains for all $L$ terminals, and $\boldsymbol{H}_{\mathrm{dl}}^{\mathrm{H}}=\left[\boldsymbol{h}_{\mathrm{dl}, 1}^{\mathrm{H}}, \ldots, \boldsymbol{h}_{\mathrm{dl}, L}^{\mathrm{H}}\right]$ is the $L \times M$ normalized fast-fading channel to all $L$ terminals from the BS. ${ }^{2}$

\footnotetext{
${ }^{2}$ Note that the channel and the link gain models are explicitly stated here for ease of exposition.
} 


\subsubsection{Downlink Linear Precoding}

For any given $M \times L$ downlink linear precoder, $\boldsymbol{W}=\left[\boldsymbol{w}_{1}, \ldots, \boldsymbol{w}_{L}\right]$, the received signal at the $l$-th terminal is given by

$$
r_{\mathrm{dl}, l}=\rho_{\mathrm{dl}}^{\frac{1}{2}} \boldsymbol{g}_{\mathrm{dl}, l}^{\mathrm{H}} \boldsymbol{W} \boldsymbol{s}_{\mathrm{dl}}+n_{\mathrm{dl}, l}=\rho_{\mathrm{dl}}^{\frac{1}{2}} \boldsymbol{g}_{\mathrm{dl}, l}^{\mathrm{H}} \boldsymbol{w}_{l} s_{\mathrm{dl}, l}+\rho_{\mathrm{dl}}^{\frac{1}{2}} \sum_{\substack{k=1 \\ k \neq l}}^{L} \boldsymbol{g}_{\mathrm{dl}, l}^{\mathrm{H}} \boldsymbol{w}_{k} s_{\mathrm{dl}, k}+n_{\mathrm{dl}, l},
$$

where $\rho_{\mathrm{dl}}$ denotes the total average transmit power at the BS array, $\boldsymbol{w}_{l}$ represents the normalized precoding vector corresponding to the the $l$-th terminal, $\boldsymbol{s}_{\mathrm{dl}}=$ $\left[s_{\mathrm{dl}, 1}, \ldots, s_{\mathrm{dl}, L}\right]^{\mathrm{T}}$ is an $L \times 1$ vector of pay load data on the downlink intended for the $L$ terminals. The data symbol desired for terminal $l$ is constrained to have unit average power, hence $\mathbb{E}\left[\left|s_{\mathrm{dl}, l}\right|^{2}\right]=1$, for $l=1,2, \ldots, L$. Moreover, $n_{\mathrm{dl}, l} \sim \mathcal{C N}\left(0, \sigma_{\mathrm{dl}, l}^{2}\right)$ models the effects of additive white Gaussian noise at the input of terminal $l$. It is assumed that $\sigma_{1}^{2}=, \ldots,=\sigma_{L}^{2}=\sigma_{\mathrm{dl}}^{2}$. Therefore, the link SNR for any given link from the BS array to a given terminal is defined as the ratio of the total average transmit power to noise power at terminal $l$, i.e., $\rho_{\mathrm{dl}} / \sigma_{\mathrm{dl}}^{2} \cdot{ }^{3}$

From (6.3), the instantaneous SINR for terminal $l$ can be written as

$$
\operatorname{SINR}_{\mathrm{dl}, l}=\frac{\rho_{\mathrm{dl}}\left|\boldsymbol{g}_{\mathrm{dl}, l}^{\mathrm{H}} \boldsymbol{w}_{l}\right|^{2}}{\sigma_{\mathrm{dl}, l}^{2}+\rho_{\mathrm{dl}} \sum_{\substack{k=1 \\ k \neq l}}^{L}\left|\boldsymbol{g}_{\mathrm{dl}, l}^{\mathrm{H}} \boldsymbol{w}_{k}\right|^{2}} .
$$

As such, the downlink spectral efficiency (measurable in bits/sec/Hz) for terminal $l$ can be written as $\mathrm{R}_{\mathrm{dl}, l}=\log _{2}\left(1+\mathrm{SINR}_{\mathrm{dl}, l}\right)$, leading to the downlink ergodic sum spectral efficiency given by

$$
\mathbb{E}\left[\mathrm{R}_{\mathrm{dl}, \mathrm{sum}}^{\boldsymbol{W}}\right]=\mathbb{E}\left[\sum_{l=1}^{L} \mathrm{R}_{\mathrm{dl}, l}\right]
$$

with the use of a specific linear processor, $\boldsymbol{W}$. The expectation here is performed over small-scale fading in the channel. Note that $\boldsymbol{W}$ can be a MF or a ZF

\footnotetext{
${ }^{3}$ Note that the definition of the link SNR is consistent with the definitions in Chapters 3 , 4, 5, 7 and 8 .
} 
precoder, in which case, (6.5) will be denoted as $\mathbb{E}\left[\mathrm{R}_{\mathrm{dl}, \text { sum }}^{\mathrm{MF}}\right]$ and $\mathbb{E}\left[\mathrm{R}_{\mathrm{dl}, \text { sum }}^{\mathrm{ZF}}\right]$, respectively. ${ }^{4}$

\subsubsection{Uplink Linear Combining}

Equivalently, on the uplink, the composite $M \times 1$ received signal at the BS can be written as

$$
\boldsymbol{y}_{\mathrm{ul}}=\rho_{\mathrm{ul}}^{\frac{1}{2}} \boldsymbol{G}_{\mathrm{ul}} \boldsymbol{s}_{\mathrm{ul}}+\boldsymbol{n}_{\mathrm{ul}}
$$

where $\rho_{\mathrm{ul}}$ is the average uplink transmit power from each terminal, $\boldsymbol{G}_{\mathrm{ul}}$ denotes the composite $M \times L$ fast-fading from $L$ terminals to $M$ BS antennas, including the link gains for all terminals, $\boldsymbol{s}_{\mathrm{ul}}=\left[s_{1, \mathrm{ul}}, s_{2, \mathrm{ul}}, \ldots, s_{L, \mathrm{ul}}\right]$ is the $L \times 1$ is the vector of data symbols on the uplink, such that the data symbol intended for terminal $l$ has unit energy, i.e., $\mathbb{E}\left[\left|s_{\mathrm{ul}, l}\right|^{2}\right]=1$. Moreover, $\boldsymbol{n}_{\mathrm{ul}}=\left[n_{\mathrm{ul}, 1}, n_{\mathrm{ul}, 2}, \ldots, n_{\mathrm{ul}, M}\right]^{\mathrm{T}}$ is the $M \times 1$ vector of additive white Gaussian noise at the BS antennas, where $n_{\mathrm{ul}, m} \sim \mathcal{C N}\left(0, \sigma_{\mathrm{ul}, m}^{2}\right)$. Note that $\sigma_{\mathrm{ul}, 1}^{2}=, \ldots,=\sigma_{\mathrm{ul}, M}^{2}=\sigma_{\mathrm{ul}}^{2}$ is assumed and the link SNR for an arbitrary link from a given terminal to the BS is denoted by the ratio of the average uplink transmit power to the noise power at the BS array, i.e., $\rho_{\mathrm{ul}} / \sigma_{\mathrm{ul}}^{2}{ }^{5}$ After linear combining, $\boldsymbol{y}_{\mathrm{ul}}$ is separated into $L$ streams with a $L \times M$ combining matrix, $\boldsymbol{T}=\left[\boldsymbol{t}_{1}, \ldots, \boldsymbol{t}_{M}\right]$, such that

$$
\boldsymbol{r}_{\mathrm{ul}}=\boldsymbol{T} \boldsymbol{y}_{\mathrm{ul}}=\rho_{\mathrm{ul}}^{\frac{1}{2}} \boldsymbol{T} \boldsymbol{G}_{\mathrm{ul}} \boldsymbol{s}_{\mathrm{ul}}+\boldsymbol{T} \boldsymbol{n}_{\mathrm{ul}}
$$

Thus, the linearly combined signal from terminal $l$ is given by

$$
r_{\mathrm{ul}, l}=\rho_{\mathrm{ul}}^{\frac{1}{2}} \boldsymbol{t}_{l} \boldsymbol{g}_{\mathrm{ul}, l} s_{\mathrm{ul}, l}+\rho_{\mathrm{ul}}^{\frac{1}{2}} \sum_{\substack{k=1 \\ k \neq l}}^{L} \boldsymbol{t}_{l} \boldsymbol{g}_{\mathrm{ul}, k} s_{\mathrm{ul}, k}+\boldsymbol{t}_{l} \boldsymbol{n}_{\mathrm{ul}},
$$

\footnotetext{
${ }^{4}$ The downlink SINR, spectral efficiency and ergodic sum spectral efficiency definitions are presented here primarily for ease of exposition. The definition is consistent with that in Chapters 3, 4 and 5 .

${ }^{5}$ Note that the definition of the link SNR here is also consistent with the definitions in Chapters 3 and 8 .
} 
resulting in the corresponding uplink SINR for terminal $l$ given by

$$
\operatorname{SINR}_{\mathrm{ul}, l}=\frac{\rho_{\mathrm{ul}}\left|\boldsymbol{t}_{l} \boldsymbol{g}_{\mathrm{ul}, l}\right|^{2}}{\sigma_{\mathrm{ul}, l}^{2}|| \boldsymbol{t}_{l}||^{2}+\rho_{\mathrm{ul}} \sum_{\substack{k=1 \\ k \neq l}}^{L}\left|\boldsymbol{t}_{l} \boldsymbol{g}_{\mathrm{ul}, k}\right|^{2}} .
$$

Equivalently, the uplink spectral efficiency for terminal $l$ can be written as $\mathrm{R}_{\mathrm{ul}, l}=$ $\log _{2}\left(1+\operatorname{SINR}_{\mathrm{ul}, l}\right)$. As such, the uplink ergodic sum spectral efficiency given by

$$
\mathbb{E}\left[\mathrm{R}_{\mathrm{ul}, \text { sum }}^{\boldsymbol{T}}\right]=\mathbb{E}\left[\sum_{l=1}^{L} \mathrm{R}_{\mathrm{ul}, l}\right]
$$

for any given type of linear combiner in $\boldsymbol{T}$, where the expectation is evaluated over the small-scale fading in the channel.

In the sections which follow, simplified closed-form approximations to the above performance metrics are presented with downlink and uplink signal processing techniques. In particular, $\mathrm{MF}$ and $\mathrm{ZF}$ precoding are considered on the downlink and MRC, ZF and MMSE combiners are considered on the uplink.

\subsection{Downlink Performance Analysis}

\subsubsection{MF Precoding Analysis}

With column-wise normalization, the MF precoding matrix, $\boldsymbol{W}_{\mathrm{MF}}$ is a scaled version of the conjugate transpose of the composite $L \times M$ downlink channel, $\boldsymbol{G}_{\mathrm{dl}}^{\mathrm{H}}$, from $M$ BS antennas to $L$ terminals. Hence, $\boldsymbol{W}_{\mathrm{MF}}=\boldsymbol{G}_{\mathrm{dl}} \boldsymbol{\eta}_{\mathrm{MF}}$, where $\boldsymbol{\eta}_{\mathrm{MF}}=\operatorname{diag}\left(\frac{1}{\left\|\boldsymbol{g}_{\mathrm{dl}, 1}\right\|}, \ldots, \frac{1}{\left\|\boldsymbol{g}_{\mathrm{dl}, L}\right\|}\right)$ is a diagonal matrix which ensures that the downlink precoding vector to each terminal (each column of $\boldsymbol{W}_{\mathrm{MF}}$ ) has unit norm. Considering this, the downlink MF SINR for terminal $l$ from (6.4) can be expressed as [146]

$$
\operatorname{SINR}_{\mathrm{dl}, l}^{\mathrm{MF}}=\frac{\rho_{\mathrm{dl}}\left\|\boldsymbol{g}_{\mathrm{dl}, l}\right\|^{2}}{\sigma_{\mathrm{dl}, l}^{2}+\boldsymbol{g}_{\mathrm{dl}, l}^{\mathrm{H}}\left(\rho_{\mathrm{dl}} \sum_{\substack{k=1 \\ k \neq l}}^{L} \frac{\boldsymbol{g}_{\mathrm{dl}, k} \boldsymbol{g}_{\mathrm{dl}, k}^{\mathrm{H}}}{\left\|\boldsymbol{g}_{\mathrm{d}, k}\right\|^{2}}\right) \boldsymbol{g}_{\mathrm{dl}, l}} .
$$


It is noteworthy that with a fixed number of user terminals and a moderately large number of BS antennas, $\sum_{\substack{k=1 \\ k \neq l}}^{L}\left(\boldsymbol{g}_{\mathrm{dl}, k} \boldsymbol{g}_{\mathrm{dl}, k}^{\mathrm{H}}\right) /\left\|\boldsymbol{g}_{\mathrm{dl}, k}\right\|^{2}$ in the denominator of (6.11) tends to stabilize numerically, and one can approximate this term with its expected value, given by $\frac{L-1}{M} \boldsymbol{I}_{L}$. Knowing this, (6.11) can now be written as

$$
\operatorname{SINR}_{\mathrm{dl}, l}^{\mathrm{MF}} \approx \frac{\rho_{\mathrm{dl}}\left\|\boldsymbol{g}_{\mathrm{dl}, l}\right\|^{2}}{\sigma_{\mathrm{dl}, l}^{2}+\rho_{\mathrm{dl}} \frac{(L-1)}{M}\left\|\boldsymbol{g}_{\mathrm{dl}, l}\right\|^{2}}=\frac{\rho_{\mathrm{dl}} \beta_{l} \chi_{M}^{2}}{\sigma_{\mathrm{dl}, l}^{2}+\rho_{\mathrm{dl}} \frac{(L-1)}{M} \beta_{l} \chi_{M}^{2}},
$$

where $\chi_{M}^{2}$ denotes a chi-squared random variable with $M$ complex degrees of freedom [147]. The expected per-terminal SINR can then be approximated via the classical first-order delta method approximation motivated in Section 3.4.3, where the expectations of numerator and denominator of (6.12) are evaluated separately. This leads to the following proposition.

Proposition 6.3.1. With MF processing and column-wise power normalization at the BS, the expected per-terminal SINR of terminal $l$, undergoing i.i.d. Rayleigh fading is approximated by

$$
\mathbb{E}\left[\mathrm{SINR}_{\mathrm{dl}, l}^{\mathrm{MF}}\right] \approx \frac{\rho_{\mathrm{dl}} \beta_{l} M}{\sigma_{\mathrm{dl}, l}^{2}+\rho_{\mathrm{dl}} \beta_{l}(L-1)}
$$

Method for Proposition 6.3.1. Taking the expected value of the numerator and denominator of (6.12) yields

$$
\begin{aligned}
\mathbb{E}\left[\operatorname{SINR}_{l}^{\mathrm{MF}}\right] \approx \frac{\mathbb{E}\left[\rho_{\mathrm{dl}} \beta_{l} \chi_{M}^{2}\right]}{\sigma_{\mathrm{dl}, l}^{2}+\mathbb{E}\left[\rho_{\mathrm{dl}} \frac{(L-1)}{M} \beta_{l} \chi_{M}^{2}\right]} \stackrel{\stackrel{(a)}{=} \frac{\rho_{\mathrm{dl}} \beta_{l} \mathbb{E}\left[\chi_{M}^{2}\right]}{\sigma_{\mathrm{dl}, l}^{2}+\rho_{\mathrm{dl}}\left[\frac{(L-1)}{M} \beta_{l}\right] \mathbb{E}\left[\chi_{M}^{2}\right]}}{ } \\
\stackrel{(b)}{=} \frac{\rho_{\mathrm{dl}} \beta_{l} M}{\sigma_{\mathrm{dl}, l}^{2}+\rho_{\mathrm{dl}} \beta_{l}(L-1)},
\end{aligned}
$$

where $(a)$ is obtained by extracting the relevant constants and $(b)$ is obtained by recognizing that $\mathbb{E}\left[\chi_{M}^{2}\right]=M$ from the standard properties of the chi-squared random variable [147], concluding the proof.

Remark 6.3.1. The derived result in (6.13) presents a remarkably simple and insightful approximation to the expected per-terminal SINR with MF processing at the BS. By inspection, it can be readily observed that with a fixed link gain at terminal $l, \beta_{l}$, the expected signal power grows with the number of 
service antennas, $M$, at the BS, while with a fixed link SNR (i.e., fixed $\rho_{\mathrm{dl}} / \sigma_{\mathrm{dl}}^{2}$ ), the expected interference power increases proportional to the total number of terminals, $L$, in the system. Moreover, it is worth noting that in the limit when $M$ and $L \rightarrow \infty$, the ratio converges to a deterministic limit, as both $M$ and $L$ grow without bound at the same rate.

The first-order delta approximation employed in (6.13) can be further improved by considering higher order terms, as mentioned in [87]. Hence, a thirdorder delta approximation is further considered here for the ratio of random variables in (6.12). This leads to the following proposition.

Proposition 6.3.2. Under the same conditions as Proposition 6.3.1, with a third-order delta approximation, the downlink expected per-terminal MF SINR for terminal $l$ is given by

$$
\mathbb{E}\left[\mathrm{SINR}_{\mathrm{dl}, l}^{\mathrm{MF}}\right] \approx \frac{\rho_{\mathrm{dl}} \beta_{l} M}{\sigma_{\mathrm{dl}, l}^{2}+\rho_{\mathrm{dl}} \beta_{l}(L-1)}-\frac{\rho_{\mathrm{dl}} \beta_{l}^{2}(L-1)}{\left[\sigma_{\mathrm{dl}, l}^{2}+\rho_{\mathrm{dl}} \beta_{l}(L-1)\right]^{3}} .
$$

Method for Proposition 6.3.2. One can begin by denoting the numerator and the denominator of (6.12) as $X$ and $Y$, respectively. Then from first principles, the $n$-th order Taylor series approximation for the function $f(x, y)$ around $\left(x_{0}, y_{0}\right)$ is given by

$$
f(x, y) \approx \sum_{i+j=0}^{n} \frac{1}{i ! j !} N^{i, j}\left(x_{0}, y_{0}\right)\left(x-x_{0}\right)^{i}\left(y-y_{0}\right)^{j}
$$

where

$$
N^{i, j}\left(x_{0}, y_{0}\right)=\left[\frac{\partial^{i+j}}{\partial x^{i} \partial y^{j}} f(x, y)\right]_{(x, y)=\left(x_{0}, y_{0}\right)} .
$$

Replacing $(x, y)$ with $(X, Y)$ and $\left(x_{0}, y_{0}\right)$ with $\left(\mu_{X}, \mu_{Y}\right)$, where $\mu_{X}=\mathbb{E}[X]$ and $\mu_{Y}=\mathbb{E}[Y]$ and performing the expectation of (6.16) results in the delta approximation to the mean of a function of two random variables. This is given by

$$
\mathbb{E}[f(X, Y)] \approx \sum_{i+j=0}^{n} \frac{1}{i ! j !} N^{i, j}(i, j)\left(\mu_{X}, \mu_{Y}\right) \mathbb{E}\left[\left(X-\mu_{X}\right)^{i}\left(Y-\mu_{Y}\right)^{j}\right]
$$


For a ratio of two random variables, $f(X, Y)=\frac{X}{Y}$, computation of $N^{i, j}\left(\mu_{X}, \mu_{Y}\right)$ gives

$$
\begin{aligned}
\mathbb{E}\left[\frac{X}{Y}\right] \approx & \frac{\mu_{X}}{\mu_{Y}}+\sum_{i=2}^{n} \frac{(-1)^{i-1}}{\left(\mu_{Y}\right)^{i}} \mathbb{E}\left[\left(X-\mu_{X}\right)\left(Y-\mu_{Y}\right)^{i-1}\right]+ \\
& \sum_{i=2}^{n} \frac{(-1)^{i}}{\left(\mu_{Y}\right)^{i+1}} \mu_{X} \mathbb{E}\left[\left(Y-\mu_{Y}\right)^{i}\right] .
\end{aligned}
$$

From (6.19), it can observed that when $n=0$, one can get the first-order delta approximation of $\mathbb{E}\left[\frac{X}{Y}\right] \approx \frac{\mathbb{E}[X]}{\mathbb{E}[Y]}$. Moreover, one can rather interestingly observe that the $n=1$ term has no effect as the correction terms involve either $\mathbb{E}\left[X-\mu_{X}\right]$ or $\mathbb{E}\left[Y-\mu_{Y}\right]$, which are zero. Furthermore, when $n=2$, one can arrive at the third-order approximation, given by

$$
\begin{aligned}
\mathbb{E}\left[\frac{X}{Y}\right] & \approx \frac{\mu_{X}}{\mu_{Y}}-\frac{\mathbb{E}\left[\left(X-\mu_{X}\right)\left(Y-\mu_{Y}\right)\right]}{\left(\mu_{Y}\right)^{2}}+\frac{\mathbb{E}\left[\left(Y-\mu_{Y}\right)^{2}\right]}{\left(\mu_{Y}\right)^{3}} \\
& =\frac{\mathbb{E}[X]}{\mathbb{E}[Y]}-\frac{\operatorname{cov}[X, Y]}{\mathbb{E}[Y]^{2}}+\frac{\mathbb{E}[X] \operatorname{var}[Y]}{\mathbb{E}[Y]^{3}}
\end{aligned}
$$

In (6.12), $X=\rho_{\mathrm{dl}} \beta_{l} \chi_{M}^{2}$ and $Y=\sigma_{\mathrm{dl}, l}^{2}+\rho_{\mathrm{dl}} \frac{(L-1)}{M} \beta_{l} \chi_{M}^{2}$. Then, using the known moments of the chi-squared random variable, one can find the necessary terms required in (6.20):

$$
\begin{gathered}
\mathbb{E}[X]=\rho_{\mathrm{dl}} \beta_{l} M, \\
\mathbb{E}[Y]=\rho_{\mathrm{dl}} \beta_{l}(L-1)+\sigma_{\mathrm{dl}, l}^{2}, \\
\operatorname{cov}[X, Y]=\rho_{\mathrm{dl}} \beta_{l}^{2}(L-1),
\end{gathered}
$$

and

$$
\operatorname{var}[Y]=\frac{\rho_{\mathrm{dl}} \beta_{l}^{2}(L-1)^{2}}{M}
$$

Substituting (6.21)-(6.24) into (6.20) and simplifying yields the desired result in (6.15).

Remark 6.3.2. Some important insights can be obtained from the presented analysis above. For instance, from (6.20) and (6.24), it is worth noting that with a fixed $L$, the variance of $Y \rightarrow 0$ as $M \rightarrow \infty$. This suggests that the approximation in (6.20) will become an equality, as $M \rightarrow \infty$ and the variance 
vanishes. This is anticipated to be particularly applicable to MU-MIMO systems with a large number of antenna elements BS. Moreover, it can be observed that with a fixed $\beta_{l}, \rho_{\mathrm{dl}}$ and $\sigma_{\mathrm{dl}, l}^{2}$, the denominator of the second order term decays quadratically as a function of $(L-1)^{2}$, whilst the numerator of the second order term grows as a function of $L-1$. A similar trend can be observed whilst inspecting the third-order term, where the numerator seems to grow as a function of $(L-1)^{2}$, in comparison to the denominator which decays cubically, proportional to $(L-1)^{3}$. The higher order terms provide a correction to the first-order approximation, particularly when the variance of $Y$ in the first-order approximation does not reduce sufficiently.

While the accuracy of (6.12), (6.13) and (6.15) is investigated in Section 6.5, from (6.12), the instantaneous cumulative distribution function (CDF) of the MF per-terminal SINR can be approximated by

$$
\begin{aligned}
\mathbb{P}\left[\operatorname{SINR}_{\mathrm{dl}, l}^{\mathrm{MF}}<x\right] & \approx \mathbb{P}\left[\frac{\rho_{\mathrm{dl}} \beta_{l} \chi_{M}^{2}}{\sigma_{\mathrm{dl}, l}^{2}+\rho_{\mathrm{dl}} \beta_{l} \chi_{M}^{2} \frac{1}{M}(L-1)}<x\right] \\
& =\mathbb{P}\left[\chi_{M}^{2}<\frac{x M \sigma_{\mathrm{dl}, l}^{2} /\left(\rho_{\mathrm{dl}} \beta_{l}\right)}{M-(L-1) x}\right] \\
& =F_{M}\left[\frac{x M \sigma_{\mathrm{dl}, l}^{2} /\left(\rho_{\mathrm{dl}} \beta_{l}\right)}{M-(L-1) x}\right]
\end{aligned}
$$

where $F_{M}[\cdot]$ is the CDF of $\chi_{M}^{2}$ given by [147]

$$
F_{M}[x]=1-e^{-x} \sum_{i=0}^{M-1} \frac{x^{i}}{i !} .
$$

Both the first and third order approximations in (6.13) and (6.15) can be translated into approximations of the ergodic sum spectral efficiency via

$$
\mathbb{E}\left[\mathrm{R}_{\mathrm{dl}, \mathrm{sum}}^{\mathrm{MF}}\right] \approx \sum_{l=1}^{L} \log _{2}\left(1+\mathbb{E}\left[\mathrm{SINR}_{\mathrm{dl}, l}^{\mathrm{MF}}\right]\right)
$$

The accuracy of the above approximation will also be numerically evaluated in Section 6.5 of the chapter. In the sequel, an equivalent the simplified analysis methodology with ZF precoding is presented. 


\subsubsection{Zero-Forcing Precoding Analysis}

Unlike MF precoding, ZF precoding ensures complete cancellation of multiuser interference, given that the number of service antennas at the BS are greater than or equal to the total number of terminals. The $M \times L \mathrm{ZF}$ precoding matrix has the form, $\boldsymbol{W}_{\mathrm{ZF}}=\boldsymbol{G}_{\mathrm{dl}}\left(\boldsymbol{G}_{\mathrm{dl}}^{\mathrm{H}} \boldsymbol{G}_{\mathrm{dl}}\right)^{-1} \boldsymbol{\eta}_{\mathrm{ZF}}$, where $\boldsymbol{\eta}_{\mathrm{ZF}}$ ensures that each column of $\boldsymbol{W}_{\mathrm{ZF}}$ has a unit norm. As ZF precoding removes multiuser interference, the per-terminal SINR now becomes the per-terminal SNR, given for terminal $l$ by $[148]^{6}$

$$
\mathrm{SNR}_{\mathrm{dl}, l}^{\mathrm{ZF}}=\frac{\rho_{\mathrm{dl}}}{\sigma_{\mathrm{dl}, l}^{2}\left[\left(\boldsymbol{G}_{\mathrm{dl}}^{\mathrm{H}} \boldsymbol{G}_{\mathrm{dl}}\right)^{-1}\right]_{l, l}},
$$

where $[\boldsymbol{A}]_{l, l}$ denotes the $(l, l)$-th entry of the matrix $\boldsymbol{A}$. The expected perterminal SNR for terminal $l$ can then be written as

$$
\mathbb{E}\left[\mathrm{SNR}_{\mathrm{dl}, l}^{\mathrm{ZF}}\right]=\mathbb{E}\left[\frac{\rho_{\mathrm{dl}}}{\sigma_{\mathrm{dl}, l}^{2}\left[\left(\boldsymbol{G}_{\mathrm{dl}}^{\mathrm{H}} \boldsymbol{G}_{\mathrm{dl}}\right)^{-1}\right]_{l, l}}\right] .
$$

In the sequel, a closed-form approximation to (6.29) is derived.

Proposition 6.3.3. With ZF precoding and column-wise power normalization at the BS, the expected per-terminal SNR of terminal $l$ undergoing i.i.d. Rayleigh fading is given by

$$
\mathbb{E}\left[\mathrm{SNR}_{\mathrm{d} l, l}^{\mathrm{ZF}}\right] \approx \frac{\rho_{\mathrm{dl}}}{\sigma_{\mathrm{dl}, l}^{2}} \beta_{l}(M-L)
$$

Method for Proposition 6.3.3. One can begin by applying the univariate special case of the first-order delta expansion. This gives,

$$
\mathrm{SNR}_{\mathrm{dl}, l}^{\mathrm{ZF}} \approx \frac{\rho_{\mathrm{dl}}}{\sigma_{\mathrm{dl}, l}^{2} \mathbb{E}\left[\left[\left(\boldsymbol{G}_{\mathrm{dl}}^{\mathrm{H}} \boldsymbol{G}_{\mathrm{dl}}\right)^{-1}\right]_{l, l}\right]}
$$

\footnotetext{
${ }^{6} \mathrm{ZF} \mathrm{SNR}$ is also defined previously in Chapters 3,4 and 5, however, in those chapters, due to the matrix normalization the dependence of the ZF SNR on the inverse of the $(l, l)$-th element disappears and gets replaced by $\operatorname{tr}\left[\left(\boldsymbol{G}_{\mathrm{dl}}^{\mathrm{H}} \boldsymbol{G}_{\mathrm{dl}}\right)^{-1}\right]$.
} 
From here, one can recognize that

$$
\begin{aligned}
\mathbb{E}\left[\left[\left(\boldsymbol{G}_{\mathrm{dl}}^{\mathrm{H}} \boldsymbol{G}_{\mathrm{dl}}\right)^{-1}\right]_{l, l}\right] & =\frac{1}{\beta_{l}} \mathbb{E}\left[\left[\left(\boldsymbol{H}_{\mathrm{dl}}^{\mathrm{H}} \boldsymbol{H}_{\mathrm{dl}}\right)^{-1}\right]_{l, l}\right] \\
& =\frac{1}{\beta_{l} L} \mathbb{E}\left[\operatorname{tr}\left[\left(\boldsymbol{H}_{\mathrm{dl}}^{\mathrm{H}} \boldsymbol{H}_{\mathrm{dl}}\right)^{-1}\right]\right] \\
& \stackrel{(a)}{=} \frac{1}{\beta_{l}(M-L)},
\end{aligned}
$$

for $M \geq L+1$, where $(a)$ is obtained by using the popular result on uncorrelated central Wishart matrices, which states [62]

$$
\mathbb{E}\left[\operatorname{tr}\left[\left(\boldsymbol{H}_{\mathrm{dl}}^{\mathrm{H}} \boldsymbol{H}_{\mathrm{dl}}\right)^{-1}\right]\right]=\frac{L}{M-L}
$$

Substituting the result from (6.32) into (6.31) yields the desired expression in (6.30), concluding the proof.

Remark 6.3.3. A number of insights can be obtained from (6.30). For instance, if the operating SNR is fixed, then the expected ZF SNR for terminal $l$ is a function of $\beta_{l}$, and the difference between $M$ and $L$. Further fixing $L$, in the limit of $M \rightarrow \infty$, then the expected ZF SNR can also be seen to grow without bound, due to the absence of multiuser interference. With a fixed $M$, growing $L$ (while satisfying $M \geq L+1$ ) decreases the expected ZF SNR to $\frac{\rho_{\mathrm{dl}}}{\sigma_{\mathrm{d} 1, l}} \beta_{l}$. This is due to growth of $\mathbb{E}\left[\left[\left(\boldsymbol{G}_{\mathrm{dl}}^{\mathrm{H}} \boldsymbol{G}_{\mathrm{dl}}\right)^{-1}\right]_{l, l}\right]$, which leads to an amplification of the expected noise power in (6.31) and in turn (6.30), respectively. The accuracy of (6.30) is numerically evaluated in Section 7.5.

Moreover, the instantaneous CDF of the ZF per-terminal SNR can now be approximated by

$$
\mathbb{P}\left[\mathrm{SNR}_{\mathrm{dl}, l}^{\mathrm{ZF}}<x\right] \approx F_{M}\left[\frac{x M}{\beta_{l}\left(\rho_{\mathrm{dl}} / \sigma_{\mathrm{dl}, l}^{2}\right)(M-L)}\right]
$$

This is consistent with similar results derived in $[144,149]$. The first-order approximation in (6.30) can be translated into an ergodic sum spectral efficiency 
over $L$ terminals by stating

$$
\mathbb{E}\left[\mathrm{R}_{\mathrm{dl}, \mathrm{sum}}^{\mathrm{ZF}}\right] \approx \sum_{l=1}^{L} \log _{2}\left(1+\mathbb{E}\left[\mathrm{SNR}_{\mathrm{dl}, l]}^{\mathrm{ZF}}\right]\right)
$$

The accuracy of the above approximation will also be presented in Section 7.5 of the chapter. In the following section, analysis for uplink MU-MIMO systems are presented with MRC, ZF and MMSE combining.

\subsection{Uplink Analysis With I.I.D. Rayleigh Fad- ing}

\subsubsection{Maximum-Ratio Combining Analysis}

Although some exact results are known for uplink MU-MIMO systems with MRC processing, they have been shown to have high analytical complexity and do not lend themselves to practical insights (see e.g., [87]). Moreover, such solutions are also numerically unstable for moderate numbers of service antennas. As a result, a simpler approach to evaluate the symbol error rate with MRC was proposed in [150]. Here, analytically friendly and numerically stable approximations are developed to evaluate the per-terminal and cell-wide performance of the system. With MRC processing, the uplink combiner is given by $\boldsymbol{T}_{\mathrm{MRC}}=\boldsymbol{G}_{\mathrm{ul}}^{\mathrm{T}}$, and the instantaneous uplink SINR for terminal $l$ can be written as [59]

$$
\mathrm{SINR}_{\mathrm{ul}, l}^{\mathrm{MRC}}=\frac{\rho_{\mathrm{ul}}\left|\boldsymbol{g}_{\mathrm{ul}, l}^{\mathrm{T}} \boldsymbol{g}_{\mathrm{ul}, l}^{*}\right|^{2}}{\sigma_{\mathrm{ul}, l}^{2} \boldsymbol{g}_{\mathrm{ul}, l}^{\mathrm{T}} \boldsymbol{g}_{\mathrm{ul}, l}^{*}+\rho_{\mathrm{ul}} \sum_{\substack{k=1 \\ k \neq l}}^{L}\left|\boldsymbol{g}_{\mathrm{ul}, k}^{\mathrm{T}} \boldsymbol{g}_{\mathrm{ul}, l}^{*}\right|^{2}} .
$$

After some algebraic manipulation, (6.36) can be written as

$$
\mathrm{SINR}_{\mathrm{ul}, l}^{\mathrm{MRC}}=\psi_{l} \boldsymbol{g}_{\mathrm{ul}, l}^{\mathrm{T}} \boldsymbol{g}_{\mathrm{ul}, l}^{*},
$$


where

$$
\psi_{l}=\frac{\rho_{\mathrm{ul}} \boldsymbol{g}_{\mathrm{ul}, l}^{\mathrm{T}} \boldsymbol{g}_{\mathrm{ul}, l}^{*}}{\boldsymbol{g}_{\mathrm{ul}, l}^{\mathrm{T}}\left(\sigma_{\mathrm{ul}, l}^{2} \boldsymbol{I}_{M}+\rho_{\mathrm{ul}} \sum_{\substack{k=1 \\ k \neq l}}^{L} \boldsymbol{g}_{\mathrm{ul}, k}^{*} \boldsymbol{g}_{\mathrm{ul}, k}^{\mathrm{T}}\right) \boldsymbol{g}_{\mathrm{ul}, l}^{*}} .
$$

The expected per-terminal MRC SINR can then be approximated by evaluating the expected value of (6.38) and $\mathbb{E}\left[\boldsymbol{g}_{\mathrm{ul}, l}^{\mathrm{T}} \boldsymbol{g}_{\mathrm{ul}, l}^{*}\right]$ separately. The result of this is expressed in the following proposition.

Proposition 6.4.1. With MRC processing at the BS, the expected uplink SINR for terminal $l$ undergoing i.i.d. Rayleigh fading can be approximated by

$$
\mathbb{E}\left[\mathrm{SINR}_{\mathrm{ul}, l}^{\mathrm{MRC}}\right] \approx \frac{\rho_{\mathrm{ul}} \beta_{l} M}{\sigma_{\mathrm{ul}, l}^{2}+\rho_{\mathrm{ul}} \sum_{\substack{k=1 \\ k \neq l}}^{L} \beta_{k}}
$$

Method for Proposition 6.4.1. One can begin by denoting the numerator and denominator of (6.38) by $\widetilde{X}$ and $\widetilde{Y}$, respectively. An approximation to the instantaneous SINR for terminal $l$ can be made by replacing $\psi_{l}$ with its expected value, i.e., $\mathbb{E}\left[\psi_{l}\right]$. The author is motivated to do this as $\frac{\widetilde{X}}{\widetilde{Y}}=\left(\frac{\widetilde{X}}{M}\right)\left(\frac{\widetilde{Y}}{M}\right)^{-1}$, where both $\left(\frac{\widetilde{X}}{M}\right)$ and $\left(\frac{\widetilde{Y}}{M}\right)$ converge to constants as $M \rightarrow \infty$, by the weak law of large numbers. Following the first-order approach outlined in (6.13), one can approximate $\mathbb{E}\left[\psi_{l}\right]$ as

$$
\mathbb{E}\left[\psi_{l}\right] \approx \frac{\rho_{\mathrm{ul}} \beta_{l} M}{\beta_{l}\left\{\operatorname{tr}\left[\sigma_{\mathrm{ul}, l}^{2} \boldsymbol{I}_{M}+\rho_{\mathrm{ul}} \sum_{\substack{k=1 \\ k \neq l}}^{L} \beta_{k} \boldsymbol{I}_{M}\right]\right\}}=\frac{\rho_{\mathrm{ul}}}{\sigma_{\mathrm{ul}, l}^{2}+\rho_{\mathrm{ul}} \sum_{\substack{k=1 \\ k \neq l}}^{L} \beta_{k}}
$$

Thus, the SINR in (6.37) can now be approximated as

$$
\mathrm{SINR}_{\mathrm{ul}, l}^{\mathrm{MRC}} \approx \mathbb{E}\left[\psi_{l}\right] \boldsymbol{g}_{\mathrm{ul}, l}^{\mathrm{T}} \boldsymbol{g}_{\mathrm{ul}, l}^{*}=\frac{\rho_{\mathrm{ul}} \beta_{l}}{\sigma_{\mathrm{ul}, l}^{2}+\rho_{\mathrm{ul}} \sum_{\substack{k=1 \\ k \neq l}}^{L} \beta_{k}} \chi_{M}^{2}
$$


As a result, the expected SINR for terminal $l$ can be approximated by

$$
\mathbb{E}\left[\mathrm{SINR}_{\mathrm{ul}, l}^{\mathrm{MRC}}\right] \approx \frac{\rho_{\mathrm{ul}} \beta_{l} M}{\sigma_{\mathrm{ul}, l}^{2}+\rho_{\mathrm{ul}} \sum_{\substack{k=1 \\ k \neq l}}^{L} \beta_{k}}
$$

yielding the desired expression. This concludes the proof.

Remark 6.4.1. The simple result in (6.42) allows one to draw some useful insights: Fixing $\rho_{\mathrm{ul}}$ and $\sigma_{l, \mathrm{ul}}^{2}$, it can be seen from (6.39) that the expected signal power (the numerator of (6.39)) grows linearly with the number of receive antennas at the BS array, $M$, while the expected interference power (the denominator of (6.39)) is governed by the sum of the interfering link gains in $\beta_{k}$, where $k=1, \ldots, L$ and $k \neq l$. Furthermore, fixing all other parameters, in the limit as $M \rightarrow \infty$, the expected MRC SINR can be seen to grow without bound.

Following from the expected SINR for terminal $l$, the uplink ergodic sum spectral efficiency over all $L$ terminals can be approximated by

$$
\mathbb{E}\left[\mathrm{R}_{\mathrm{ul}, \mathrm{sum}}^{\mathrm{MRC}}\right] \approx \sum_{l=1}^{L} \log _{2}\left(1+\mathbb{E}\left[\mathrm{SINR}_{\mathrm{ul}, l}^{\mathrm{MRC}}\right]\right)
$$

while the instantaneous CDF can be approximated by

$$
\mathbb{P}\left[\mathrm{SINR}_{\mathrm{ul}, l}^{\mathrm{MRC}}<x\right] \approx F_{M}\left[x\left(\sigma_{\mathrm{ul}, l}^{2}+\rho_{\mathrm{ul}} \sum_{\substack{k=1 \\ k \neq l}}^{L} \beta_{k}\right) / \rho_{\mathrm{ul}} \beta_{l}\right] .
$$

In the subsections which follow, a simplified analysis with ZF and MMSE detectors is presented.

\subsubsection{Zero-Forcing Combining Analysis}

The uplink and downlink performance metrics for ZF processing are the identical, with the exception that $\boldsymbol{G}_{\mathrm{ul}}^{\mathrm{H}}$ is the composite downlink channel, whereas $\boldsymbol{G}_{\mathrm{ul}}$ is the uplink composite channel. Hence, the analysis methodology outlined in Section 6.3.2 can be re-used, where the same results and conclusions hold. To prove this, numerical results in Section 6.5 demonstrate the accuracy of the 
downlink and uplink ZF processing, respectively.

\subsubsection{Minimum-Mean-Squared-Error Combining Analysis}

Now, an MMSE combiner is considered at the BS array, where minimizing the mean-squared-error between the true transmitted symbol, $s_{\mathrm{ul}, l}$, for $l=1, \ldots, L$, and the output of the MMSE detector leads to the combining matrix, $\boldsymbol{T}_{\mathrm{MMSE}}=$ $\left(\boldsymbol{G}_{\mathrm{ul}}^{\mathrm{T}} \boldsymbol{G}_{\mathrm{ul}}^{*}+\frac{\sigma_{\mathrm{ul}}^{2}}{\rho_{\mathrm{ul}}} \boldsymbol{I}_{L}\right)^{-1} \boldsymbol{G}_{\mathrm{ul}}^{\mathrm{T}}$, assuming $\sigma_{\mathrm{ul}}^{2}=\sigma_{\mathrm{ul}, l}^{2}=\ldots=\sigma_{\mathrm{ul}, M}^{2}$. Following the methodology in [151], the SINR for terminal $l$ with MMSE processing can be expressed as

$$
\mathrm{SINR}_{\mathrm{ul}, l}^{\mathrm{MMSE}}=\boldsymbol{g}_{\mathrm{ul}, l}^{\mathrm{T}}\left(\frac{\sigma_{\mathrm{ul}, l}^{2}}{\rho_{\mathrm{ul}}} \boldsymbol{I}_{M}+\boldsymbol{G}_{\mathrm{ul}, l}^{*} \boldsymbol{G}_{\mathrm{ul}, l}^{\mathrm{T}}\right)^{-1} \boldsymbol{g}_{\mathrm{ul}, l}^{*},
$$

where $\boldsymbol{G}_{\mathrm{ul}, l}^{\mathrm{T}}=\left[\boldsymbol{g}_{\mathrm{ul}, 1}^{\mathrm{T}}, \ldots, \boldsymbol{g}_{\mathrm{ul}, l-1}^{\mathrm{T}}, \boldsymbol{g}_{\mathrm{ul}, l+1}^{\mathrm{T}}, \ldots, \boldsymbol{g}_{\mathrm{ul}, L}^{\mathrm{T}}\right]$ is the $(L-1) \times M$ composite matrix of all uplink channels excluding the channel from the $l$-th terminal to the BS array. In the sequel, a simplified approximation to the expected per-terminal SINR with MMSE combining is presented.

Proposition 6.4.2. With MMSE processing at the BS, the expected uplink SINR for terminal $l$ undergoing i.i.d. Rayleigh fading can be approximated by

$$
\mathbb{E}\left[\mathrm{SINR}_{\mathrm{ul}, l}^{\mathrm{MMSE}}\right] \approx c M \beta_{l}
$$

where

$$
c=\frac{\sigma_{\mathrm{ul}}^{2}}{\rho_{\mathrm{ul}} M}\left[(M-L+1)+\sum_{\substack{k=1 \\ k \neq l}}^{L}\left(1+M \beta_{k}\right)^{-1}\right] .
$$

Method for Proposition 6.4.2. One can begin by recognizing that the expected value of the inverse in (6.45) can be written as

$$
\mathbb{E}\left[\left(\frac{\sigma_{\mathrm{ul}}^{2}}{\rho_{\mathrm{ul}}} \boldsymbol{I}_{M}+\boldsymbol{G}_{\mathrm{ul}, l}^{*} \boldsymbol{G}_{\mathrm{ul}, l}^{\mathrm{T}}\right)^{-1}\right]=c \boldsymbol{I}_{M},
$$

where $c$ is a constant derived in the sequel. Taking the trace of the equality in 
(6.48) yields

$$
c M=\mathbb{E}\left[\operatorname{tr}\left[\left(\frac{\sigma_{\mathrm{ul}}^{2}}{\rho_{\mathrm{ul}}} \boldsymbol{I}_{M}+\boldsymbol{G}_{\mathrm{ul}, l}^{*} \boldsymbol{G}_{\mathrm{ul}, l}^{\mathrm{T}}\right)^{-1}\right]\right]=\frac{\sigma_{\mathrm{ul}}^{2}}{\rho_{\mathrm{ul}}}(M-(L-1))+\mathbb{E}\left[\sum_{k=1}^{L-1} \lambda_{k}^{-1}\right]
$$

since $M-(L-1)$ eigenvalues of $\frac{\sigma_{\mathrm{ul}}^{2}}{\rho_{\mathrm{ul}}} \boldsymbol{I}_{M}+\boldsymbol{G}_{\mathrm{ul}, l}^{*} \boldsymbol{G}_{\mathrm{ul}, l}^{\mathrm{T}}$ are $\frac{\sigma_{\mathrm{ul}}^{2}}{\rho_{\mathrm{ul}}}$ and the largest $L-1$ eigenvalues are denoted by $\lambda_{1}, \lambda_{2}, \ldots, \lambda_{L-1}$. However, as $\lambda_{1}, \lambda_{2}, \ldots, \lambda_{L-1}$ are also the eigenvalues of $\frac{\sigma_{\mathrm{ul}}^{2}}{\rho_{\mathrm{ul}}} \boldsymbol{I}_{L-1}+\boldsymbol{G}_{\mathrm{ul}, l}^{\mathrm{T}} \boldsymbol{G}_{\mathrm{ul}, l}^{*}$, we can express (6.49) as

$$
c M=\frac{\sigma_{\mathrm{ul}}^{2}}{\rho_{\mathrm{ul}}}(M-L+1)+\mathbb{E}\left[\operatorname{tr}\left[\left(\frac{\sigma_{\mathrm{ul}}^{2}}{\rho_{\mathrm{ul}}} \boldsymbol{I}_{L-1}+\boldsymbol{G}_{\mathrm{ul}, l}^{*} \boldsymbol{G}_{\mathrm{ul}, l}^{\mathrm{T}}\right)^{-1}\right]\right] .
$$

It is worth noting that $\frac{1}{M} \boldsymbol{G}_{\mathrm{ul}, l}^{*} \boldsymbol{G}_{\mathrm{ul}, l}^{\mathrm{T}}$ converges to $\operatorname{diag}\left(\beta_{1}, \ldots, \beta_{l-1}, \beta_{l+1}, \ldots, \beta_{L}\right)$, an $(L-1) \times(L-1)$ diagonal matrix of link gains, as $M \rightarrow \infty$. Using this fact in $(6.50)$ results in

$$
c=\frac{\sigma_{\mathrm{ul}}^{2}}{\rho_{\mathrm{ul}}}\left[(M-L+1)+\sum_{\substack{k=1 \\ k \neq l}}^{L}\left(1+M \beta_{k}\right)^{-1}\right] .
$$

Hence, replacing the inverse in (6.45) by $c \boldsymbol{I}_{M}$, where $c$ is as given in (6.51) allows one to express the MMSE SINR for terminal $l$ as

$$
\mathrm{SINR}_{\mathrm{ul}, l}^{\mathrm{MMSE}} \approx c \beta_{l} \chi_{M}^{2}
$$

As the density of $\chi_{M}^{2}$ is known in closed-form, the instantaneous CDF can also be approximated as

$$
\mathbb{P}\left[\mathrm{SINR}_{\mathrm{ul}, l}^{\mathrm{MMSE}}<x\right] \approx F_{M}\left[\frac{x}{c \beta_{l}}\right]
$$

Evaluating the expected value of (6.53) yields the desired result in (6.46).

Remark 6.4.2. To the best of the author's knowledge, the result derived in (6.46) is the simplest approximation available in the literature on uplink MU-MIMO systems with MMSE processing. The authors in [59] present a distributional approximation to the MMSE SINR in (6.45), where it was shown 
to be approximated via the gamma distribution, where its parameters are derived. The authors in $[124,151]$ present exact closed-form analysis of the MMSE SINR. However, the complexity of the analysis methodology and the resulting expressions may limit their use in performance analysis and evaluation of uplink MU-MIMO systems with moderate numbers of BS antennas. Naturally, the numerical evaluation of the derived expressions in $[59,124,151]$ will yield similar results, so long as the numerical parameters in each case are consistent.

The ergodic sum spectral efficiency over $L$ terminals with MMSE processing can now be approximated by

$$
\mathbb{E}\left[\mathrm{R}_{\mathrm{ul}, \mathrm{sum}}^{\mathrm{MMSE}}\right] \approx \sum_{l=1}^{L} \log _{2}\left(1+\mathbb{E}\left[\mathrm{SINR}_{\mathrm{ul}, l}^{\mathrm{MMSE}}\right]\right)
$$

\subsubsection{Extensions to More General Fading Channels}

It is worth noticing that the simplified analysis methodology, although presented for i.i.d Rayleigh fading channels, has wider applicability to other types of statistical fading channels. As an example, consider the uplink MRC SINR for terminal $l$, approximated by $\mathbb{E}\left[\psi_{l}\right] \boldsymbol{g}_{\mathrm{ul}, l}^{\mathrm{T}} \boldsymbol{g}_{\mathrm{ul}, l}^{*}$, as shown in (6.41). In the presence of spatially correlated Ricean fading channels, the approximate SINR with MRC processing is proportional to $\boldsymbol{g}_{\mathrm{ul}, l}^{\mathrm{T}} \boldsymbol{g}_{\mathrm{ul}, l}^{*}$, which is a non-central complex quadratic form in Gaussian random variables [152]. Moreover, $\psi_{l}$ in (6.37) depends on moments of similar random variables which can be computed. Therefore, the same approximation approach can be applied to the above mentioned channels, where exact analytical results are usually intractable. This approach is shown to give excellent numerical results for a spatially correlated Ricean fading channel with equal correlation matrices for each terminal. These numerical results are presented in Section 6.5 of the chapter. Furthermore, it is worth mentioning that an important topic for future work is to extend the simplified analysis methodology to more complex, Saleh-Valenzuela type channel models, which better capture finite numbers of propagation paths and scattering clusters in the channel, especially at higher carrier frequencies. Due to the generality of 
such models, for a given antenna topology, they consider the transmit and receive far-field array steering responses in both the azimuth and the elevation domains. Here, the analysis of uplink and downlink MU-MIMO performance metrics will be extremely cumbersome, if not intractable. The simplified approximation methodology may have a role in reducing the complexity of the resulting expressions for the performance metrics described above in Sections 6.3 and 6.4 .

The subsequent section presents the numerical results from the derived approximations against those resulting from simulations for both uplink and downlink MU-MIMO systems.

\subsection{Numerical Results and Discussion}

A circular cell of radius $100 \mathrm{~m}$ is considered, in which $L$ terminals were uniformly distributed with respect to the cell area. The BS is located at the origin of the circular cell and a $10 \mathrm{~m}$ exclusion radius is designed. ${ }^{7}$ Unless otherwise specified, all terminals experience uncorrelated i.i.d. Rayleigh fading. The large-scale fading effects are modeled as shown in (6.2), where the attenuation exponent $\alpha=3.7$ and the shadow-fading standard deviation $\sigma_{\mathrm{sf}}=8 \mathrm{~dB}$ [15]. To evaluate the accuracy of the simplified expressions, the simulations considered comprise of the evaluation of cell-wide (where each trial corresponds to a different drop) and instantaneous (where one drop is considered and the variation is over fastfading only) per-terminal SINRs, as well as downlink ergodic sum-rates for $10^{4}$ independent trials. For both downlink and uplink performance metrics, unit noise power is assumed at the receive end of a particular link, i.e., $\sigma_{\mathrm{dl}}^{2}=\sigma_{\mathrm{ul}}^{2}=$ $\sigma_{\mathrm{dl}, l}^{2}=\sigma_{\mathrm{ul}, m}^{2}=1$, for all $l=1, \ldots, L$ and $m=1, \ldots, M$. Note that the parameter $v$ is chosen such that the fifth percentile value of the instantaneous downlink and uplink SINR equals $0 \mathrm{~dB}$, post-processing, for the baseline system dimensions of $M=30$ and $L=3$. This is chosen as the baseline case as it satisfies $\frac{M}{L}=10$, a common scenario quoted in the recent literature. Furthermore,

\footnotetext{
${ }^{7}$ Note that this is the reference distance, $d_{0}$ in (6.2).
} 


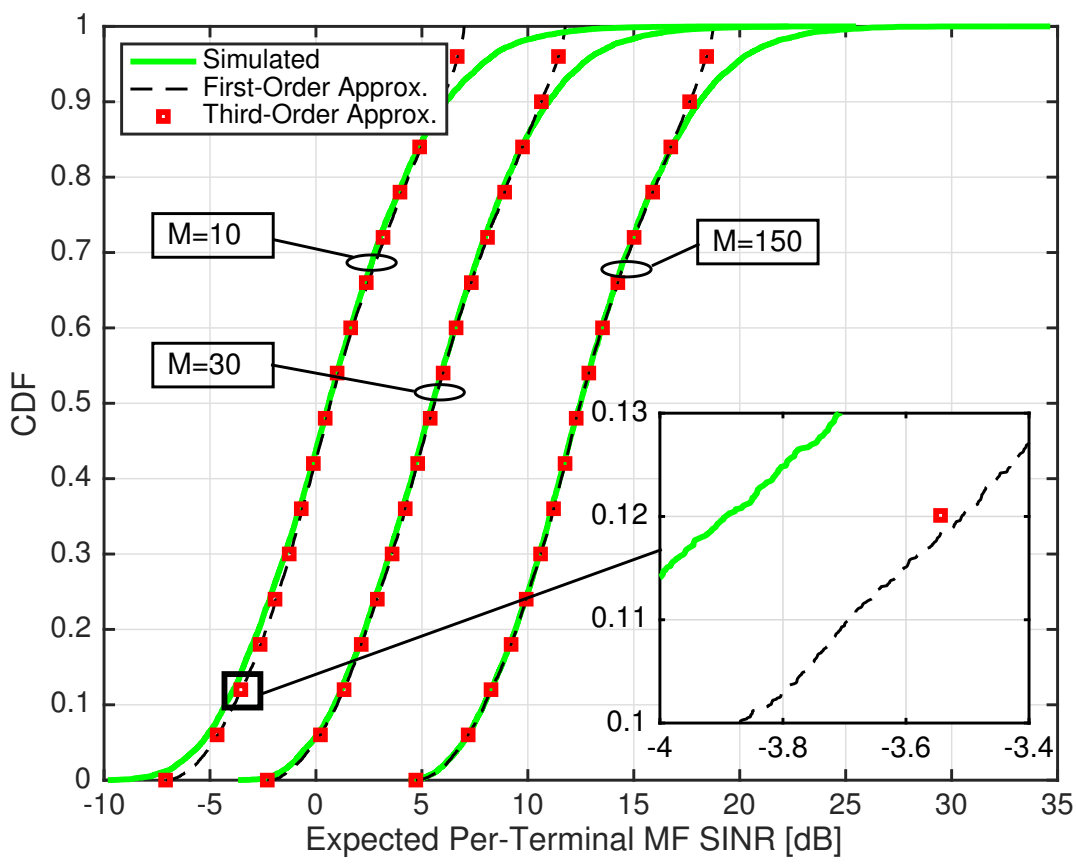

Figure 6.1: Downlink cell-wide MF expected per-terminal SINR for $M=$ 10,30, 150 with $L=3$ at link $\mathrm{SNR}=0 \mathrm{~dB}$.

30 service antennas at the BS is a moderate number, well short of large MUMIMO system dimensions. Unless otherwise specified, the link SNRs on the uplink and downlink are chosen to be $0 \mathrm{~dB}$. For ease of notation, link SNRs in the subsequent numerical results are referred to as SNRs.

Fig. 6.1 demonstrates the cell-wide downlink expected per-terminal CDF with MF precoding, where $M=10,30,150$ and $K=3$. The numerical simulations were generated using (6.11), while the approximations were generated using (6.13) and (6.15). Naturally, an increase in the expected per-terminal SINR is observed when $M$ is scaled up from 10 to 150 with $L$ remaining fixed. The third-order approximation is shown to perform better than the simple firstorder approximation for all system dimensions. For smaller system dimensions, such as the $M=10, L=3$ case, the error in the approximations and their simulated counterparts is larger at lower probabilities. However, the analytical expressions are tighter as $M$ increases. At high probabilities (above 0.9), divergence from the simulated results is observed for all cases. This is due to replacing the sum in the denominator of (6.13) by its mean value. High SINRs 


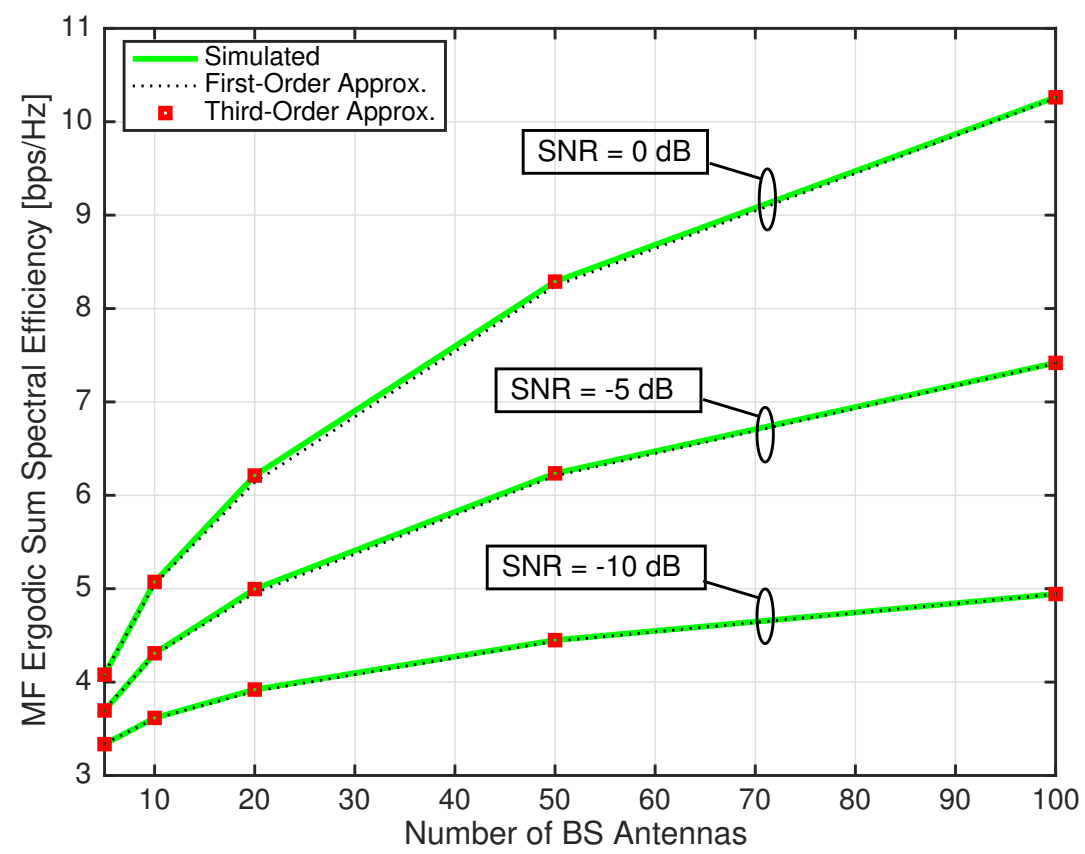

Figure 6.2: Downlink cell-wide MF ergodic sum spectral efficiency for $M=30$ and $L=3$.

occur when the denominator (interference plus noise) is small and the use of the mean value prevents some of the variation in the interference term which can lead to high SINRs. Improving the approximation at high SINRs is a topic for future research. Hence, an extremely useful estimate of cell-wide performance is obtained from the remarkably simple approach of generating multiple drops whilst for each drop using the mean SINR in (6.13). Note that the case of $M=150$ was chosen as an upper bound to analyze the approximation accuracy and strictly belongs in the large MU-MIMO category.

The expected per-terminal MF SINR was translated to an ergodic sum spectral efficiency over all terminals present in the system, using (6.10). This is demonstrated in Fig. 6.2. The approximated ergodic sum spectral efficiency approximation was evaluated from (6.27). It can be readily observed, that across all link SNR levels considered, the derived approximations remain extremely tight and numerically stable. Moreover, it is worth noting that the logarithm in the ergodic sum spectral efficiency expression helps to provide extra averaging, such that the simplified approximations are tight even for lower numbers 


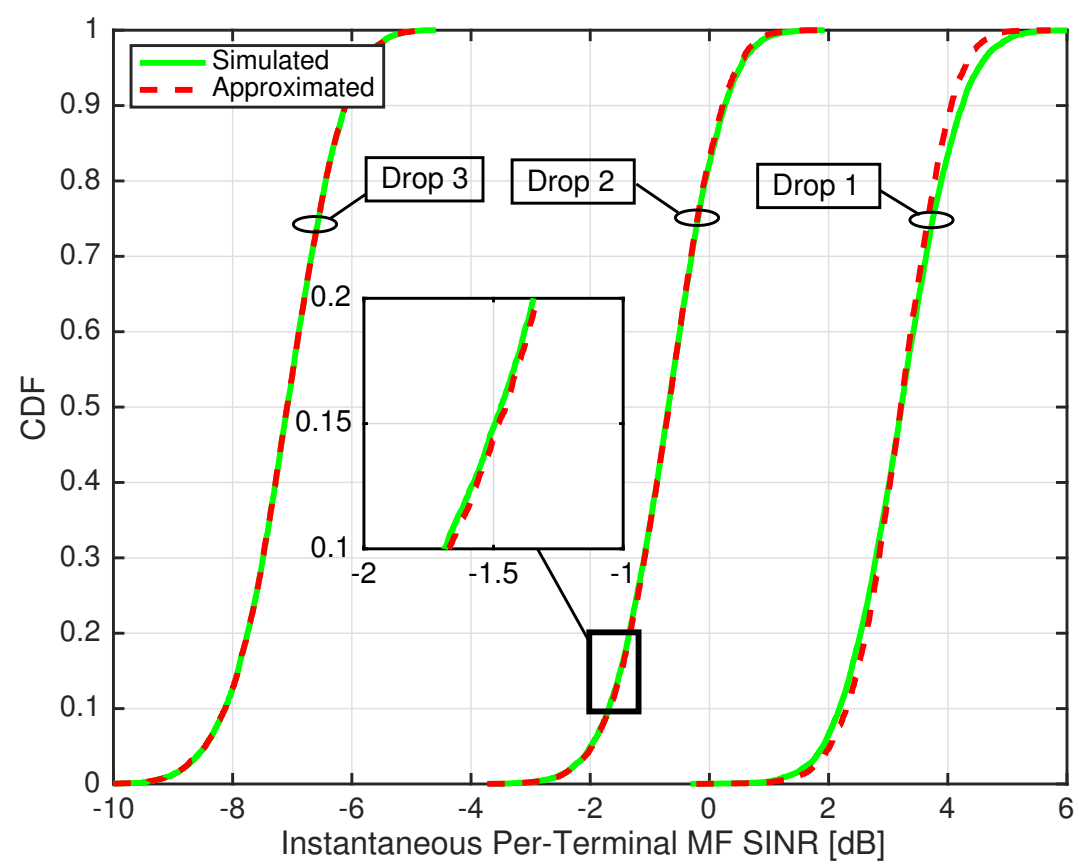

Figure 6.3: Downlink instantaneous MF SINR performance for $M=30$ and $L=3$ with link SNR $=0 \mathrm{~dB}$.

of service antennas. In contrast to the cell-wide performance, the instantaneous SINR performance with the derived CDF approximation in (6.25) is presented in Fig. 6.3 for $M=30$ and $L=3$. Here, three different drops are considered, and hence drop 2 and drop 3 result in lower instantaneous SINR performance than drop 1. One can observe that the chi-squared distributional approximation is numerically stable and accurate across all random drops of the terminals.

The ZF downlink precoding performance is now investigated. As the downlink and uplink ZF performance metrics are identical, the subsequent results are also valid for the uplink MU-MIMO equivalent, as indicated on the results themselves. Fig. 6.4 presents the cell-wide expected ZF SNR with $M=10,30$ and 150 with a fixed number of user terminals, $L=3$. The simulated cell-wide performance was evaluated by taking the expected value of (6.28), while the approximated performance was evaluated with (6.30). As $M$ transitions from being small to moderate to large, the derived uplink and downlink approximations are seen to get tighter, especially at the lower tail of the CDFs (below 0.1 probability). Overall, an excellent agreement between the approximated 


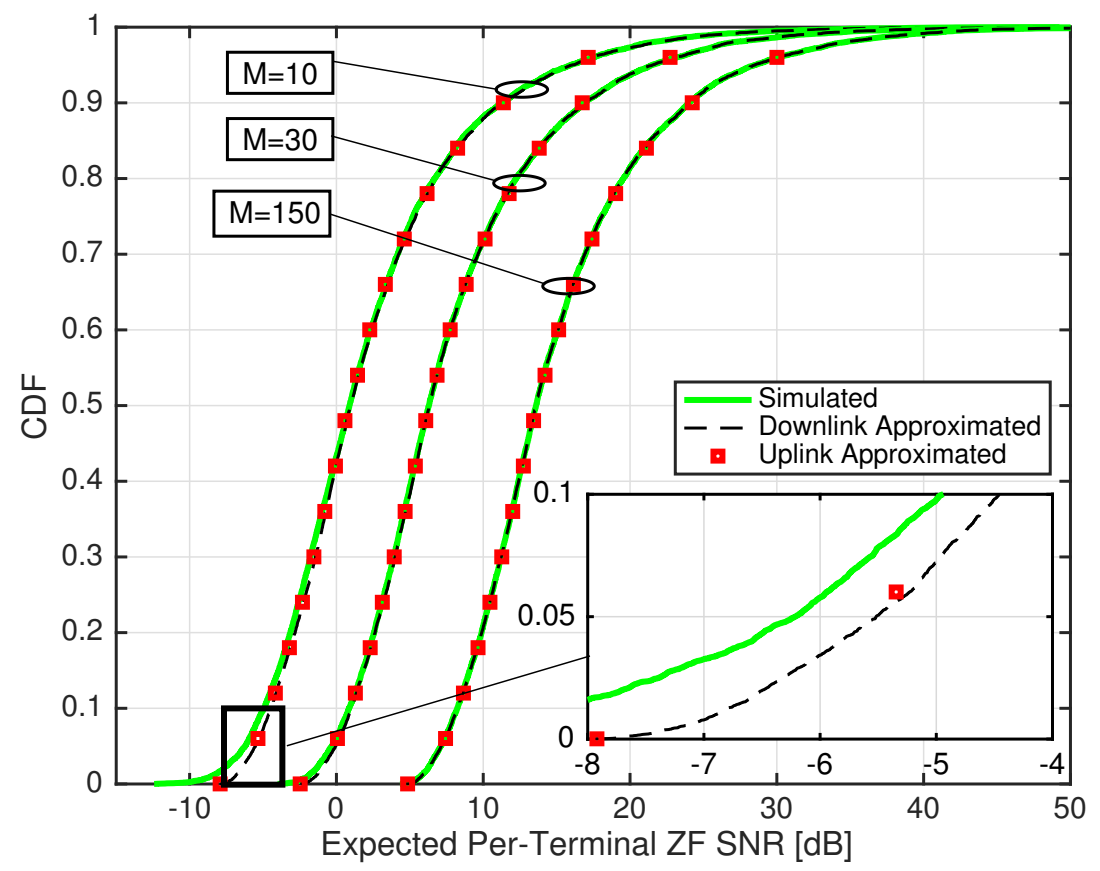

Figure 6.4: Downlink cell-wide ZF expected per-terminal SNR for $M=$ 10,30, 150 with $L=3$ at link $\mathrm{SNR}=0 \mathrm{~dB}$.

and simulated performance is observed, that is numerically stable for moderate number of antennas, for both the uplink and downlink cases, respectively. The approximated expected per-terminal ZF can be translated into a ZF ergodic sum spectral efficiency via (6.34) across all $L$ terminals. This accuracy of ergodic sum spectral efficiency across low and high link SNR levels is depicted in Fig. 6.5. In addition to this, the instantaneous SNR performance approximated in (6.33) is demonstrated in Fig. 6.6 across three different drops. As can be seen from the figure, once again the chi-squared distributional approximation seems to be extremely tight for $M=30 \mathrm{BS}$ and $L=3$ terminals, for both the uplink and downlink cases.

Now the accuracy of the uplink MRC SINR results is evaluated. Fig. 6.7 depicts the cell-wide expected per-terminal SINR performance for MRC with $M=30$ and $L=3$. As is readily observed, the proposed approximation in (6.42) is very accurate for i.i.d. Rayleigh fading. Furthermore, the generality of the approximation method is explored for semi-correlated Ricean fading with a fixed Rice factor of $5 \mathrm{~dB}$ to all terminals, for simplicity. The specular component of the 


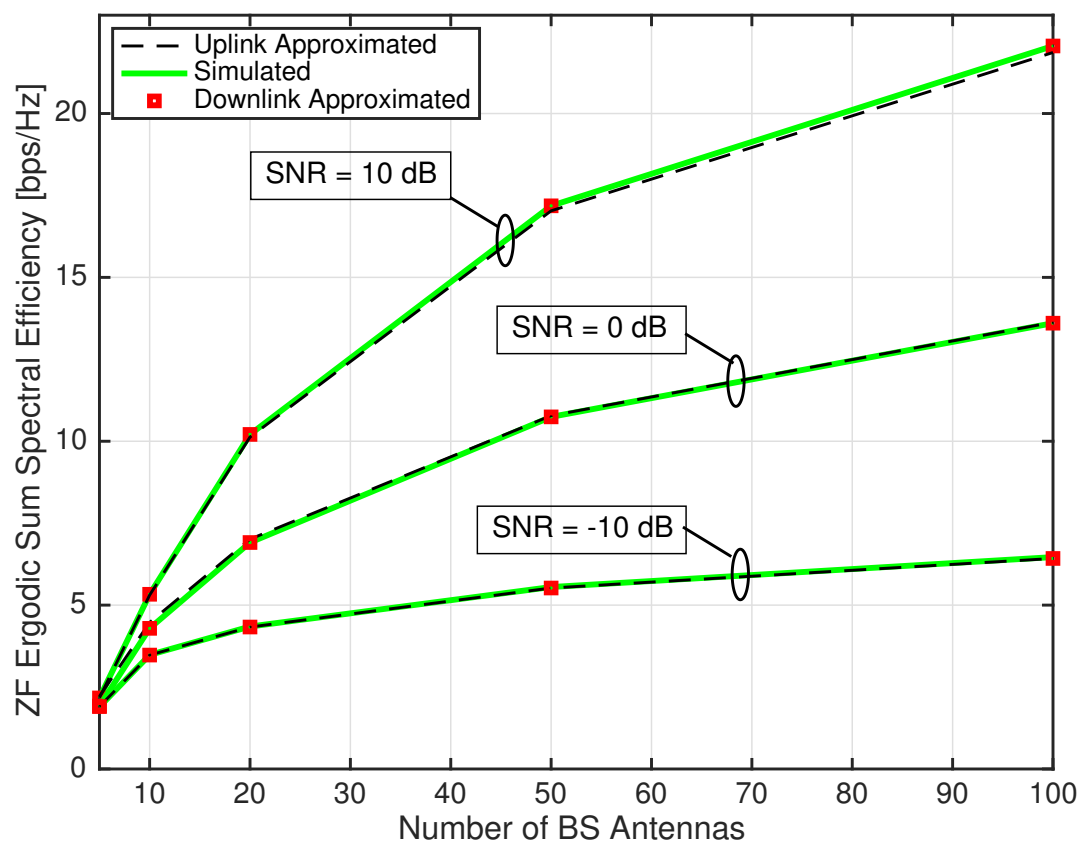

Figure 6.5: Downlink cell-wide ZF ergodic sum spectral efficiency for $M=30$ and $L=3$.

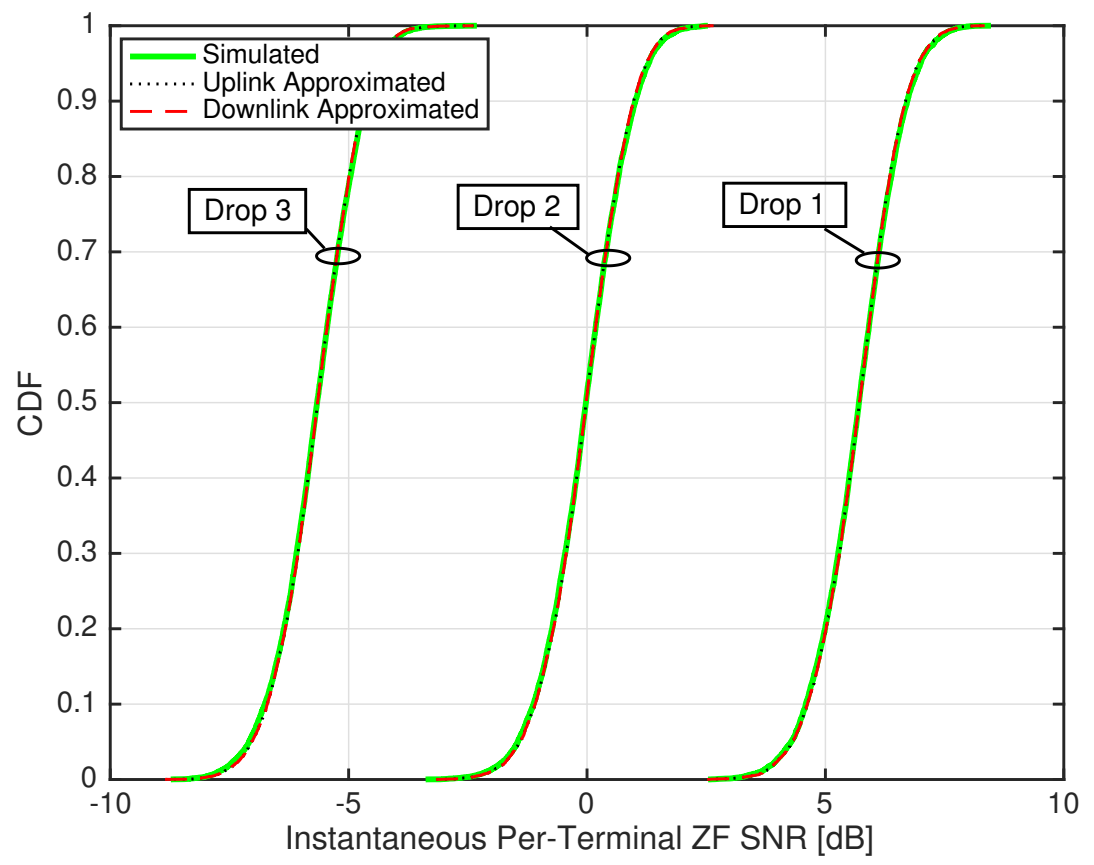

Figure 6.6: Downlink instantaneous ZF SNR performance for $M=30$ and $L=3$ with link $\mathrm{SNR}=0 \mathrm{~dB}$.

channel is governed by a uniform linear array transmit steering vector response, while a fixed transmit correlation matrix is assumed for each terminal following 


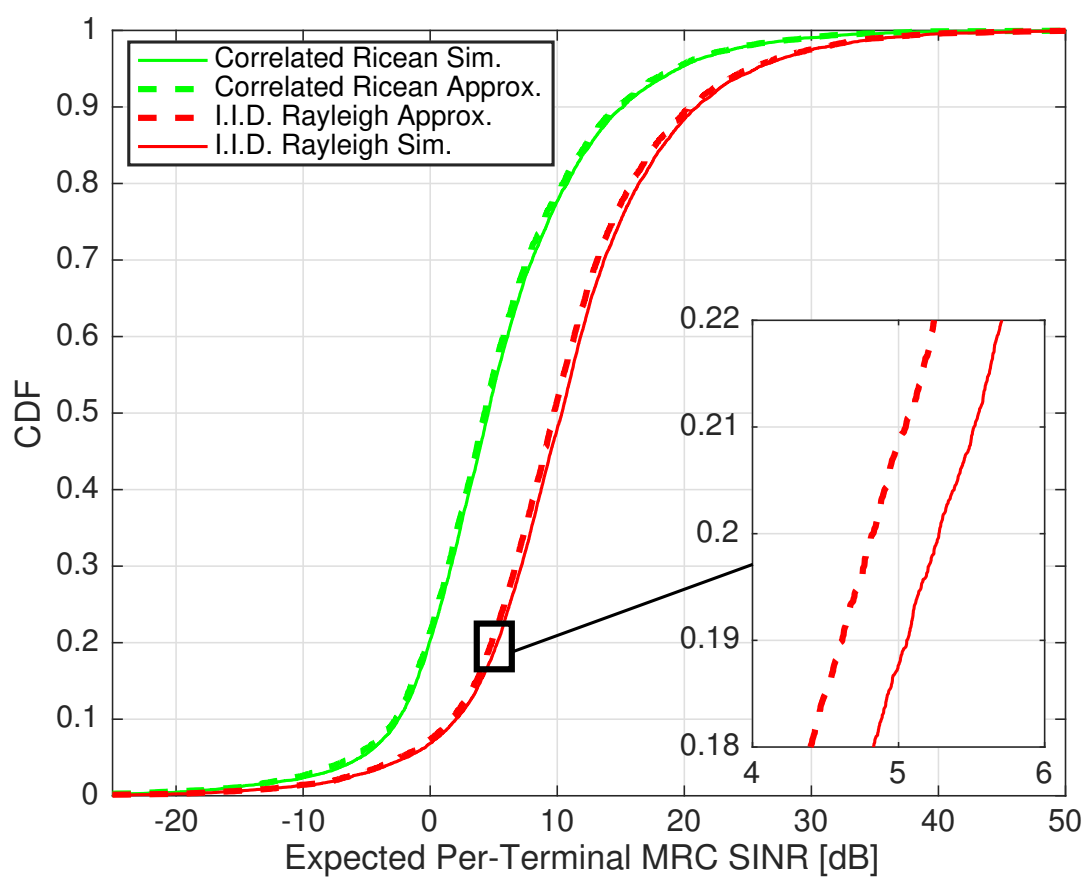

Figure 6.7: Uplink cell-wide expected per-terminal MRC SINR for $M=30$ and $L=3$ at link $\mathrm{SNR}=0 \mathrm{~dB}$.

the classical exponential model, where the spatial correlation co-efficient was chosen to be $0.9[46,80]$. The approximation is seen to remain tight even in the presence of strong correlation and LoS, demonstrating its robustness to other propagation models.

The instantaneous and expected per-terminal SINR performance was also evaluated for the MMSE combiner, as shown in Fig. 6.8 and 6.9 using (6.46) and (6.52), respectively. The accuracy of the approximation is seen to increase with increasing $M$. This has a direct relation to the fact that $\frac{1}{M}\left[\boldsymbol{G}_{\mathrm{ul}, l}^{*} \boldsymbol{G}_{\mathrm{ul}, l}^{\mathrm{T}}\right]$ tends to stabilize as $M$ gets larger. Hence, cell-wide performance to a complex metric like the MMSE detector can be investigated by the remarkably simple result in (6.46), which gives straightforward insights into the impact of system and propagation parameters. The instantaneous CDF approximation in (6.52) is shown in Fig. 6.9 for three drops, where the simulated per-terminal SINR closely follows the approximated SINR. Hence, not only can accurate cell-wide approximations be developed, but simple instantaneous SINR distributions for a single drop can also be accurately approximated for a complex performance 


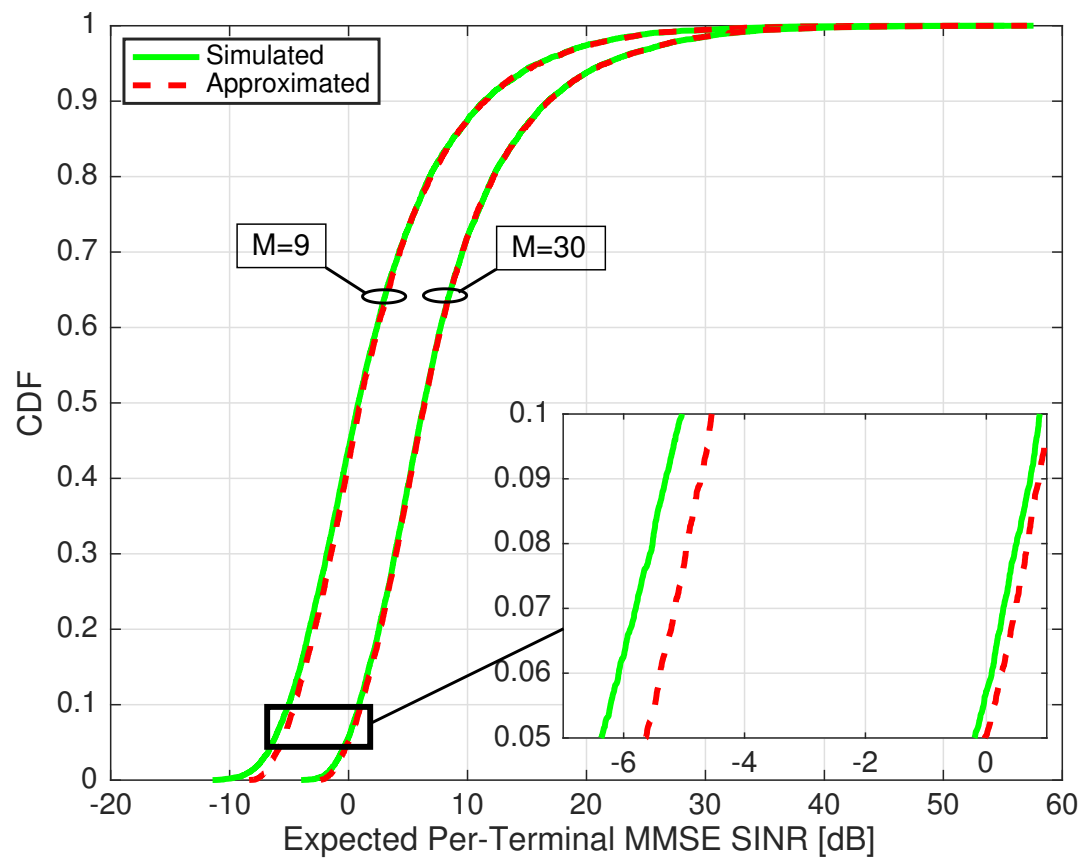

Figure 6.8: Uplink cell-wide expected per-terminal MMSE SINR for $M=9,30$ and $L=3$ at link SNR $=0 \mathrm{~dB}$.

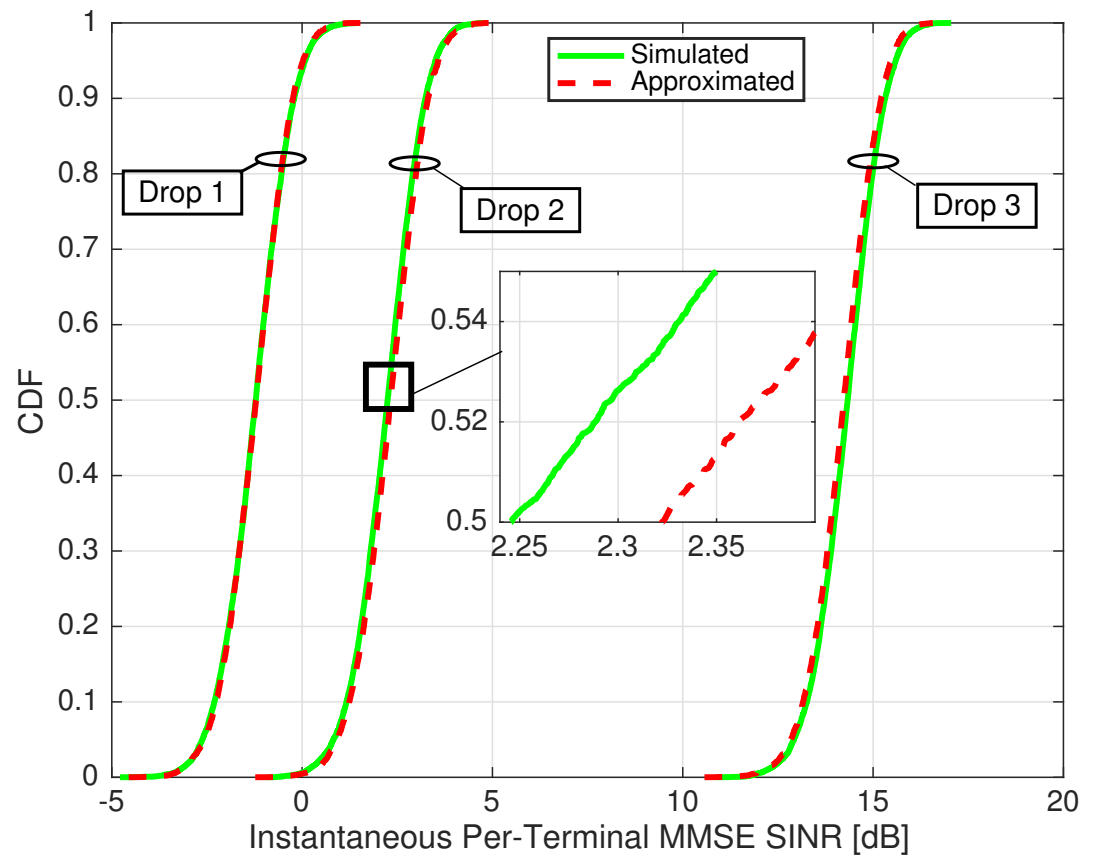

Figure 6.9: Uplink instantaneous MMSE SINR performance for $M=30$ and $L=3$ with link SNR $=0 \mathrm{~dB}$.

metric. 


\subsection{Concluding Remarks}

In this chapter, simplified approximations to the instantaneous and expected per-terminal SINR and SNR were derived for uplink and downlink MU-MIMO systems and a general class of linear transceivers operating with moderate numbers of BS antennas. The instantaneous and expected SINR approximations were translated to instantaneous and ergodic sum spectral efficiencies for the range of performance metrics considered. It was demonstrated that the cellwide and single-drop performance for a wide range of metrics can be predicted with practically useful accuracy using the remarkably simple approximations. The possibility of such general and simple MU-MIMO performance analysis is largely missing in the literature. The results are also shown to remain reasonably accurate even for conventional MU-MIMO dimensions. The approximation methodology is easily adaptable to other types of statistical fading channel models. An example of this was demonstrated with uplink MRC processing, where the expected per-terminal cell-wide SINR was extended to spatially correlated Ricean fading channels and was shown to maintain good accuracy. 


\section{Chapter 7}

\section{Linear Precoding Analysis With Unequal Spatial Correlation}

Summary. It is well known that spatial correlation across an antenna array is detrimental to the terminal signal-to-interference-plus-noise-ratio (SINR), as well as system spectral efficiency. For a multiuser multiple-input multiple-output (MU-MIMO) system, it is mathematically proven that the widely used, yet overly simplified, spatial correlation models with common correlation structures for each terminal underestimate the expected SINR and ergodic sum spectral efficiency. As a result, such models act as a lower bound on the resulting performance. In stark contrast to this, the use of more complex, yet physically motivated, remote scattering models results in unequal spatial correlation structures for each terminal, leading to higher performance. Assuming semi-correlated Rayleigh fading, with matched-filter (MF) and zero-forcing (ZF) transmit precoding, closed-form approximations to the expected SINR, expected signal-tonoise-ratio (SNR) and ergodic sum spectral efficiency of a MU-MIMO system are derived. The derived expressions provide clear insights into the impact of unequal spatial correlation matrices. The expressions are robust to changes in the system dimensions, link SNRs and unequal spatial correlation levels. In addition to the above, the derived approximations remain accurate for all nonphysical and physical correlation models considered. Overall, the results from this chapter demonstrate the sensitivity of the achievable performance using 
different spatial correlation models. Such a sensitivity analysis is useful in providing a cautionary tale of its impact to potential analysts, who may not have access to expensive empirical channel measurements.

\subsection{Introduction}

The focus of this chapter is on the downlink of a multiuser multiple-input multiple-output (MU-MIMO) system, where a base station (BS) equipped with a transmit antenna array simultaneously serves multiple single antenna user terminals in the same time-frequency interval $[9,57]$. In contemporary cellular geometries, the BS is typically located on a tower or on a relatively tall building. In this case, propagation between the BS and a given terminal usually occurs via a large number of scattering clusters [137]. ${ }^{1}$ These clusters are usually seen from the BS with a narrow angular spread, and such types of propagation channels can be statistically characterized by correlated Gaussian random variables [41]. ${ }^{2}$ Having said this, propagation channel vectors of different terminals, which are physically separated by many multiples of the carrier wavelength ${ }^{3}$ are typically mutually independent in a statistical sense [41].

Indeed, spatially correlated channels with multiple transmit and receive antennas have been well characterized for a variety of transmit correlation models (see for example the seminal work of the authors in [21,154-158]). In a MU-MIMO context, it is well known that transmit correlation is a detrimental source to the per-terminal signal-to-interference-plus-noise-ratio (SINR) and

\footnotetext{
${ }^{1}$ Note that this is a general remark. Naturally, the propagation between the BS and terminals depends on many other physical factors. For instance, the operating carrier frequency, which ultimately governs how far the electromagnetic waves can propagate through the environment. Operating a cellular system at very high carrier frequencies, such as in those in the millimeter-wave (mmWave) frequency bands, may not allow the BS to "see" a large number of scattering clusters, in comparison to lower frequency bands $[137,153]$, due to the increase in the level of sparsity in the radio channel. However, it is to be noted that experimental verification of this fact is rather limited, since the rotating horn antennas typically used for channel measurements have a coarse angular resolution, so that the determination of the number of multipath components is not easily possible.

${ }^{2}$ It should be emphasized for the sake of clarity that this comment particularly applies for radio channels with a large number of scattering clusters.

${ }^{3}$ In the microwave frequency band, for a typical carrier frequency between $2-5 \mathrm{GHz}$, the channel wavelength is between 15 and $6 \mathrm{~cm}$, respectively.
} 
system spectral efficiency, causing power loss, mostly in the high link signalto-noise-ratio (SNR) regime, as demonstrated in $[65,80,89,93,112,159]$. This finding is typically reported when all terminals in the system have a common correlation structure (i.e., identical spatial correlation matrices) [80]. In contrast to this, a different line of investigation has identified that transmit correlation can in fact improve MU-MIMO system performance (see for example $[42,117,120,160-163])$. The critical observation from these studies is that in a multiuser environment, when the variations in the local terrain (scattering) around multiple user terminals are considered, there exists a diverse range of spatial correlation patterns. This means that in addition to the close proximity of serving antenna elements at the BS, each terminal's correlation matrix will be significantly influenced by the specific terrain geometry around the terminal.

Overall, differences in the transmit correlation patterns indicate differences in the large-scale (or long-term) dominant (preferential) directions of the propagation channel to each terminal. This difference is largely a function of two physical quantities: (1) the geometry of the local scattering environment around the terminals' geographical location and ${ }^{4}(2)$ inter-element spacing between successive serving antenna elements at the transmit array [14, 40, 43, 117]. Hence, the diverse nature of the transmit correlation patterns can be investigated in the MU-MIMO framework to explore the differences on the terminal SINR and system spectral efficiency, in comparison to when each terminal has a common correlation structure. In order to fully exploit the above mentioned physical phenomena, remote scattering models have been proposed for modeling spatial correlation structures. For a given propagation environment, these models are generally parameterized in terms of the central azimuth direction-ofarrival (DoA) to the terminal, angular spread in the azimuth domain, and interelement spacing at the serving array. Typical examples of such models are the one-ring correlation model with a uniform DoA distribution in the azimuth domain [43] and the Laplacian DoA correlation model [117]. These models

\footnotetext{
${ }^{4}$ The assumption here is that this also encapsulates any dominant line-of-sight (LoS) direction in the propagation channel. Due to this reason, the LoS direction is not explicitly stated as a key contributor to unequal correlation.
} 
are a result of extensive channel measurements in urban propagation environments, both in the microwave and millimeter-wave (mmWave) frequency bands $[128,130,151,164,165]$.

In addition to the above, with a large number of serving antennas at the $\mathrm{BS}$, the work of [41-43] imposes a tall unitary structure on the correlation matrices, resulting in the joint spatial division and multiplexing (JSDM) precoding approach. Furthermore, for time-division duplex multicellular systems, the groundbreaking work of the authors in $[37,166,167]$ has reported that the use of remote scattering models with disjoint (non-overlapping) angular support sets eliminates the fundamental impairment of pilot contamination, associated with the reuse of uplink pilot sequences across multiple cells. ${ }^{5}$ Nevertheless, it remains to be seen just how much performance gain diversity in the correlation matrices across multiple terminals offer, when considering different remote scattering models. Currently, there exists no mathematical quantification for this difference. Moreover, there also does not exist a rigorous performance comparison between the most common remote scattering models and the simple, yet non-physical equal correlation models. Hence, having an intuitive understanding of the performance sensitivity using different spatial correlation models is important, as it allows researchers to select correlation models in a more informed manner, especially in multiuser systems. This is the focus of the chapter.

Assuming perfect channel state information at the serving array with matchedfilter (MF) and zero-forcing (ZF) precoding, the expected per-terminal SINR, expected per-terminal SNR, as well as ergodic sum spectral efficiency gains of the MU-MIMO system with unequal correlation matrices is explored. Closedform approximations of the above mentioned performance metrics are derived. It is worth mentioning that with large numbers of BS antennas, limiting approximations for the terminal SINR and system spectral efficiency with ZF and MF precoding and terminal specific correlation (unequal) matrices have been

\footnotetext{
${ }^{5}$ Ever since the breakthrough of large antenna arrays by Marzetta (in [9]), it was believed that pilot contamination is an impairment which will not vanish in the limit of an unlimited number of service antennas. However, the authors in the above mentioned studies have shown that this belief is incorrect, and it is an artifact from using simplistic channel models, such as the uncorrelated, independent and identically distributed (i.i.d.) Rayleigh fading.
} 
analyzed in $[43,60,116]$. However, the majority of the analytically derived expressions with $\mathrm{ZF}$ and MF are left as numerical solutions in terms of fixed point algorithms, making it extremely difficult to gain any practical insights and intuition into the behavior of unequal correlation matrices. On this basis, developing an insightful analysis of linear precoded MU-MIMO systems with unequal correlation matrices is also an open problem, which is tackled in this chapter. With clear insights into the contributing network parameters, our closed-form expressions demonstrate the impact of unequal correlation matrices on the above performance metrics. Moreover, the analysis is capable of handling a variety of commonly used physical and non-physical transmit correlation structures (discussed further in the text and in Section 7.5.1) in the channel.

More specifically, the contributions of this chapter are as follows:

1. Tight closed-form approximations to the expected per-terminal SINR, expected per-terminal SNR and the ergodic sum spectral efficiency with MF and ZF precoding are derived. Considering spatially correlated Rayleigh fading channels with transmit correlation, the closed-form expressions provide clear insights into the impact of various network parameters, such as the number of serving antennas, the number of user terminals, link SNR and unequal correlation matrices, respectively.

2. For both MF and ZF, it is mathematically proven that for a fixed average correlation matrix across all terminals, equal correlation matrices increase the total interference power in comparison to the case where each terminal has a unique correlation matrix. Due to this reason, equal correlation matrices provide a useful lower limit in the achievable performance of such systems.

3. The numerical findings suggest that for both MF and ZF precoding, the choice of a particular correlation model has a significant impact on the expected SINR, expected SNR and ergodic sum spectral efficiency. Physically motivated models, such as the one-ring [41,43] and Laplacian [117] give enhanced performance in comparison to the non-physical models, such 
as the exponential $[46,80]$ and the Clerckx $[120,121]$ correlation models. Moreover, the closed-form expressions are robust to changes in system dimensions, link SNR and remain tight with a all considered unequal correlation models. For ease of reference, the one-ring model is referred to as (O.R.) and the Laplacian model is referred to as (Lap.) for the remainder of the chapter.

The rest of the chapter is organized as follows: Section 7.2 presents a further discussion on the typical spatial correlation scenarios for multiuser systems. Section 7.3 presents the system model and performance metrics used throughout the chapter with MF and ZF downlink precoding. Section 7.4 demonstrates the expected per-terminal MF SINR, expected per-terminal ZF SNR and ergodic sum spectral efficiency approximations and discusses the implications of these results. Section 7.5 presents the numerical results and evaluates the accuracy of the derived results in Section 7.4. Note that Section 7.5 also presents discussion on the various spatial correlation models considered in the chapter and the thesis. Section 7.6 outlines the concluding remarks of the chapter.

\subsection{Typical Spatial Correlation Scenarios}

In the case where the serving array is able to see the same set of scattering clusters for all terminals, a constant correlation matrix is valid [47]. To illustrate this, one can take an example of the classical Jakes scattering, where the serving array is surrounded by a uniform ring of scatterers. Then, the resultant correlation matrices are simply governed by a zeroth-order Bessel function of the first kind [47]. ${ }^{6}$ Naturally, this is a more sensible model on the terminal side of the link, which may be in dense clutter [41,43,117].

In contrast to this, if the serving array illuminates a distinct (unique) set of scatterers, as captured by the remote scattering models, unequal correlation matrices occur with wide variations in the correlation patterns for different terminals. Here, differences in the terminal locations relative to the serving array

\footnotetext{
${ }^{6}$ The argument of the Bessel function contains $2 \pi d$, where $d$ is the inter-element spacing between two successive antenna elements normalized by the carrier wavelength.
} 
cause differences in the level of local scattering around each terminal. Nevertheless, the discussion thus far only captures the two extreme cases, where the serving array is able to see either the same or disjoint (unique) set of scattering clusters for each terminal. In reality, it is likely that some scatterers may be common to a subset of user terminals, and distinct to others. This scenario is depicted in Fig. 7.1, where the BS is equipped with a $M$ element uniform linear array (ULA) and is shown to simultaneously serve two terminals. ${ }^{7}$ The terminal closer to the broadside of the array may experience a different level of scattering in comparison to the terminal closer the endfire of the array. Irrespective of this fact, in both cases, distinct scatterers may be illuminated within the vicinity of both terminals' physical locations, as depicted in the green dots. This further creates a power variations, known as angular spread, demonstrated with red circles around the terminals. ${ }^{8}$ These contribute to the inequality of the correlation structures for the two terminals. In addition to this, a common scatterer (in the form of a building) is shared amongst the two terminals, increasing the level of similarity in the correlation matrices between the terminals. This is demonstrated via the dotted green lines.

This line of thinking raises the following fundamental question: Assuming a fixed propagation environment, how far apart do two terminals have to be to see distinct scatterers being illuminated? Answering this question requires extensive channel measurements, which is beyond the scope of this chapter and the thesis. Nevertheless, some experimental validation to the above discussion is presented in a recent study carried out by the authors of [75]. In this study, results of a mmWave MU-MIMO channel measurement campaign are presented at $60 \mathrm{GHz}$ in downtown Helsinki, Finland. It is reported that with a $8 \times 8$ uniform planar array, where the inter-element spacing does not exceed $\lambda_{c}$, the wavelength at the operating carrier frequency, on average only 2 scattering clusters out of

\footnotetext{
${ }^{7}$ In order not to cluter the diagram, the concept is illustrated with ray optics, a rather simplistic model for electromagnetic propagation, from a reference antenna element $m$. In reality, there are electromagnetic waves which radiate in wavefronts from each antenna elements.

${ }^{8}$ The author wishes to provide another cautionary tale here in that the literature quotes many definitions of the angular spread. For the sake of this chapter (and the thesis), the author refers to the term angular spread as the intra-cluster angular spread in the azimuth domain.
} 


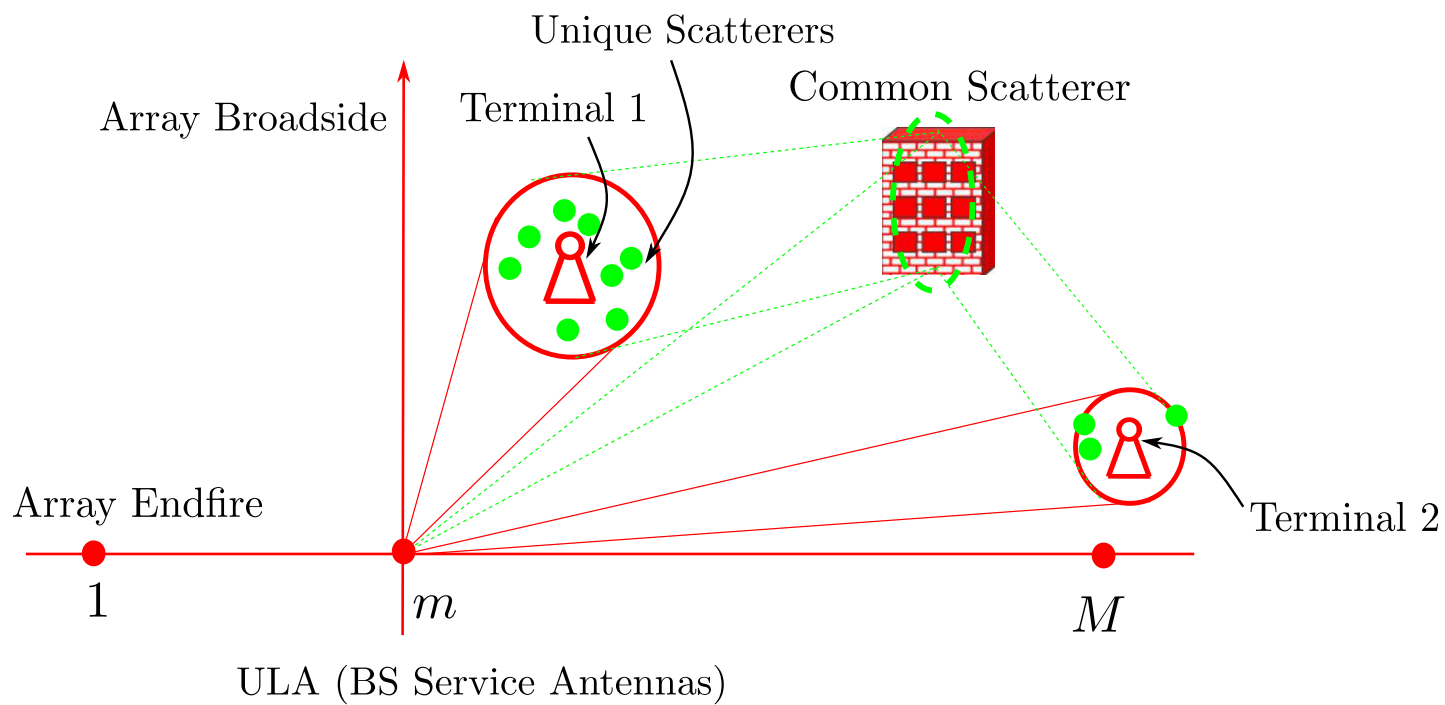

Figure 7.1: A birds eye view of a downlink MU-MIMO propagation scenario.

a total of 10 were shared between two single-antenna terminals spaced $4 \mathrm{~m}$ apart. This is in comparison to when the terminals are $1 \mathrm{~m}$ apart, nearly 8 scattering clusters were observed to be common. To achieve completely different correlation patterns at the two terminals, inter-user distance of approximately 20 $\mathrm{m}$ is reported. Moreover, the average number of common scattering clusters as a function of the inter-user separation was reported to decrease exponentially, and increasing the array size to $16 \times 16$ with the same inter-element spacing further reduced the level of correlation in the propagation channel, due to increased spatial diversity. The above mentioned scenarios are explored in this chapter and analytical results are derived considering MF and ZF downlink precoding. The following section presents the MU-MIMO system model considered in the chapter.

\subsection{System Model}

\subsubsection{Signal Model}

The downlink of a MU-MIMO system is considered. The BS is equipped with a ULA consisting of $M$ ideal, omnidirectional transmit antenna elements, simultaneously serving $L$ non-cooperative, single-antenna terminals $(M \geq L)$ in 
the same time-frequency interval. No mechanical downtilt at the array is assumed. Narrow-band transmission with equal power allocation to each terminal is assumed. With channel knowledge at the BS, the received signal at the $l$-th terminal can be written as ${ }^{9}$

$$
r_{\mathrm{dl}, l}=\rho_{\mathrm{dl}}^{\frac{1}{2}}\left(\frac{\beta_{l}}{\eta}\right)^{\frac{1}{2}} \boldsymbol{g}_{\mathrm{dl}, l}^{\mathrm{H}} \boldsymbol{w}_{l} s_{\mathrm{dl}, l}+\rho_{\mathrm{dl}}^{\frac{1}{2}} \sum_{\substack{k=1 \\ k \neq l}}^{L}\left(\frac{\beta_{l}}{\eta}\right)^{\frac{1}{2}} \boldsymbol{g}_{\mathrm{dl}, l}^{\mathrm{H}} \boldsymbol{w}_{k} s_{\mathrm{dl}, k}+n_{\mathrm{dl}, l}
$$

where $\rho_{\mathrm{dl}}$ is the average downlink transmit power at the BS, $\beta_{l}$ is the link gain of the $l$-th terminal (discussed in the next paragraph). The $1 \times M$ downlink small-scale fading channel to terminal $l$ is denoted by $\boldsymbol{g}_{\mathrm{dl}, l}^{\mathrm{H}}$. As this chapter considers semi-correlated Rayleigh fading with spatial correlation at the BS array, $\boldsymbol{g}_{\mathrm{dl}, l}^{\mathrm{H}}=\boldsymbol{h}_{\mathrm{d} 1, l}^{\mathrm{H}} \boldsymbol{R}_{l}^{\frac{1}{2}}$, where $\boldsymbol{h}_{\mathrm{dl}, l}^{\mathrm{H}} \sim \mathcal{C N}\left(0, \boldsymbol{I}_{M}\right)$ and $\boldsymbol{R}_{l}$ is the $M \times M$ transmit spatial correlation matrix specific to terminal $l$. While the discussion of the particular structure of $\boldsymbol{R}_{l}$ is postponed to to Section 7.5, the presented channel model allows one to analyze any type of antenna correlation structure in $\boldsymbol{R}_{l}$. Furthermore, $\boldsymbol{w}_{l}$ is the $M \times 1$ un-normalized downlink precoding vector from the BS to terminal $l$, obtained from the $l$-th column of $\boldsymbol{W}$, the $M \times L$ composite un-normalized precoding matrix. Note that $s_{\mathrm{dl}, l}$ is the downlink normalized data symbol desired for the $l$-th terminal, such that $\mathbb{E}\left[\left|s_{\mathrm{d} l, l}\right|^{2}\right]=1$. Moreover, $n_{\mathrm{dl}, l} \sim \mathcal{C N}\left(0, \sigma_{\mathrm{dl}, l}^{2}\right)$ models the effects of additive white Gaussian noise at the $l$-th terminal with noise power $\sigma_{\mathrm{dl}, l}^{2}$. Following [80], $\eta=\|\boldsymbol{W}\|_{\mathrm{F}}^{2} / L$ is the normalization parameter for downlink precoding, such that the overall transmit power remains unchanged (discussed in the following section).

The link gain for terminal $l$ takes the form of

$$
\beta_{l}=v\left(\frac{d_{0}}{d_{l}}\right)^{\alpha} \zeta_{l}
$$

and composes of the large-scale fading effects of geometric attenuation and shadow-fading, specific to terminal $l .{ }^{10}$ In particular, $v$ is the constant for

\footnotetext{
${ }^{9}$ For ease of exposition, the key system equations and performance metrics are repeated here and are consistent with Chapters 3,5 and 6 .

${ }^{10}$ This is also stated for ease of exposition and is consistent with Chapters 3,4,5 and 6 .
} 
geometric attenuation at a reference distance $d_{0}, d_{l}$ is the link distance between the BS array and terminal $l, \alpha$ is the attenuation exponent and $\zeta_{l}$ models the effects of shadow-fading with a log-normal density, i.e., $10 \log _{10}\left(\zeta_{k}\right) \sim \mathcal{N}\left(0, \sigma_{\text {sf }}^{2}\right)$, where $\sigma_{\mathrm{sf}}$ is the shadow-fading standard deviation. The corresponding value of each parameter is defined in Section 7.5. For the remainder of the chapter, the link SNR is defined as the ratio of $\rho_{\mathrm{dl}}$ with $\sigma_{\mathrm{dl}}^{2}$ for any arbitrary link, i.e. $\rho_{\mathrm{dl}} / \sigma_{\mathrm{dl}}^{2}$, where $\sigma_{\mathrm{dl}}^{2}=\sigma_{\mathrm{dl}, 1}^{2}=\ldots=\sigma_{\mathrm{dl}, L}^{2}$. For simplicity, it is assumed that $\sigma_{\mathrm{dl}}^{2}=1$.

Next, the MF SINR, ZF SNR and ergodic sum spectral efficiency performance metrics are introduced.

\subsubsection{MF SINR, ZF SNR and Ergodic Sum Spectral Efficiency}

With MF precoding, $\boldsymbol{w}_{l}$, the $l$-th column of the composite $M \times L$ precoding matrix, $\boldsymbol{W}^{\mathrm{MF}}=\boldsymbol{G}_{\mathrm{dl}}$ is chosen to maximize the desired signal power at the l-th terminal. Here, $\boldsymbol{G}_{\mathrm{dl}}^{\mathrm{H}}=\left[\boldsymbol{g}_{\mathrm{dl}, 1}, \ldots, \boldsymbol{g}_{\mathrm{dl}, L}\right]^{\mathrm{H}}$ is the $L \times M$ composite matrix containing downlink channels for all $L$ terminals in the system. From (7.1), the SINR for $l$-th terminal can be written as

$$
\operatorname{SINR}_{\mathrm{dl}, l}^{\mathrm{MF}}=\frac{\rho_{\mathrm{dl}} \frac{\beta_{l}}{\eta^{\mathrm{MF}}}\left|\boldsymbol{g}_{\mathrm{dl}, l}^{\mathrm{H}} \boldsymbol{w}_{l}\right|^{2}}{\sigma_{\mathrm{dl}, l}^{2}+\rho_{\mathrm{dl}} \frac{\beta_{l}}{\eta^{\mathrm{MF}}} \sum_{\substack{k=1 \\ k \neq l}}^{L}\left|\boldsymbol{g}_{\mathrm{dl}, l}^{\mathrm{H}} \boldsymbol{w}_{k}\right|^{2}},
$$

where $\eta^{\mathrm{MF}}=\left\|\boldsymbol{W}^{\mathrm{MF}}\right\|_{\mathrm{F}}^{2} / L=\operatorname{tr}\left[\boldsymbol{G}_{\mathrm{dl}} \boldsymbol{G}_{\mathrm{dl}}^{\mathrm{H}}\right] / L$. On the other hand, with ZF precoding, the per-terminal SINR translates into a per-terminal SNR due to its ability to null multiuser interference. Here, $\boldsymbol{w}_{l}$ forms the $l$-th column of the $M \times L$ precoding matrix $\boldsymbol{W}^{\mathrm{ZF}}=\boldsymbol{G}_{\mathrm{dl}}\left(\boldsymbol{G}_{\mathrm{dl}}^{\mathrm{H}} \boldsymbol{G}_{\mathrm{dl}}\right)^{-1}$. Upon recognizing that $\boldsymbol{G}_{\mathrm{dl}}^{\mathrm{H}} \boldsymbol{W}^{\mathrm{ZF}}=\boldsymbol{G}_{\mathrm{dl}}^{\mathrm{H}} \boldsymbol{G}_{\mathrm{dl}}\left(\boldsymbol{G}_{\mathrm{dl}}^{\mathrm{H}} \boldsymbol{G}_{\mathrm{dl}}\right)^{-1}=\boldsymbol{I}_{L}$, the ZF SNR at the $l$-th terminal is given by

$$
\mathrm{SNR}_{\mathrm{dl}, l}^{\mathrm{ZF}}=\frac{\rho_{\mathrm{dl}} \beta_{l}}{\sigma_{\mathrm{dl} l, l}^{2} \eta^{\mathrm{ZF}}}=\frac{\rho_{\mathrm{dl}} \beta_{l}}{\sigma_{\mathrm{dl}, l}^{2}\left\{\frac{1}{L}\left\{\operatorname{tr}\left[\left(\boldsymbol{G}_{\mathrm{dl}}^{\mathrm{H}} \boldsymbol{G}_{\mathrm{dl}}\right)^{-1}\right]\right\}\right\}}
$$


with $\eta^{\mathrm{ZF}}=\left\|\boldsymbol{W}^{\mathrm{ZF}}\right\|_{\mathrm{F}}^{2} / L=\operatorname{tr}\left[\left(\boldsymbol{G}_{\mathrm{dl}}^{\mathrm{H}} \boldsymbol{G}_{\mathrm{dl}}\right)^{-1}\right] / L$. The received SINR with MF precoding in (7.3) can be translated into the an ergodic sum spectral efficiency (measurable in bits/seconds/Hz) of the system for all $L$ terminals. This is given by

$$
\mathbb{E}\left[\mathrm{R}_{\text {sum,dl }}^{\mathrm{MF}}\right]=\mathbb{E}\left[\sum_{l=1}^{L} \log _{2}\left(1+\operatorname{SINR}_{\mathrm{dl}, l}^{\mathrm{MF}}\right)\right] .
$$

Replacing $\mathrm{SINR}_{\mathrm{dl}, l}^{\mathrm{MF}}$ with $\mathrm{SNR}_{\mathrm{dl}, l}^{\mathrm{ZF}}$ yields the ergodic sum spectral efficiency, $\mathbb{E}\left[\mathrm{R}_{\mathrm{sum}, \mathrm{dl}}^{\mathrm{ZF}}\right]$, with ZF precoding. Note that for both $\mathbb{E}\left[\mathrm{R}_{\mathrm{sum}, \mathrm{dl}}^{\mathrm{MF}}\right]$ and $\mathbb{E}\left[\mathrm{R}_{\mathrm{sum}, \mathrm{dl}}^{\mathrm{ZF}}\right]$, the statistical expectation is performed over the small-scale fading in the downlink channel.

In the following section, tight analytical approximations to the expected perterminal SINR, expected per-terminal SNR and ergodic sum spectral efficiency are derived for both MF and ZF precoding.

\subsection{Analysis Methodology and Implications}

\subsubsection{Expected MF SINR and Ergodic Sum Spectral Ef- ficiency}

From (7.3), the expected SINR for terminal $l$ can be obtained by performing $\mathbb{E}\left[\mathrm{SINR}_{\mathrm{dl}, l}^{\mathrm{MF}}\right]$. As motivated in Chapter 3, Section 3.4.3, the exact evaluation of $\mathbb{E}\left[\mathrm{SINR}_{\mathrm{dl}, l}^{\mathrm{MF}}\right]$ is extremely challenging $[68,80,86,87,93]$. Hence, a first-order delta expansion is again employed, as shown in the analysis methodology of the above references. This allows one to decouple the numerator and the denominator of (7.3), such that the expected MF SINR for terminal $l$ can be approximated by

$$
\mathbb{E}\left[\mathrm{SINR}_{\mathrm{dl}, l}^{\mathrm{MF}}\right] \approx \frac{\rho_{\mathrm{dl}} \frac{\beta_{l}}{\tilde{\eta}^{\mathrm{MF}}} \mathbb{E}\left[\left|\boldsymbol{g}_{\mathrm{dl}, l}^{\mathrm{H}} \boldsymbol{w}_{l}\right|^{2}\right]}{\sigma_{\mathrm{dl}, l}^{2}+\rho_{\mathrm{dl}} \frac{\beta_{l}}{\tilde{\eta}^{\mathrm{MF}}} \sum_{\substack{k=1 \\ k \neq l}}^{L} \mathbb{E}\left[\left|\boldsymbol{g}_{\mathrm{dl}, l}^{\mathrm{H}} \boldsymbol{w}_{k}\right|^{2}\right]},
$$

where $\tilde{\eta}^{\mathrm{MF}}=\mathbb{E}\left[\eta^{\mathrm{MF}}\right]$. In the following lemmas, we derive the expectations in the numerator and denominator of (7.6).

Lemma 7.4.1. When $\boldsymbol{g}_{\mathrm{dl}, l}^{\mathrm{H}}=\boldsymbol{h}_{\mathrm{dl}, l}^{\mathrm{H}} \boldsymbol{R}_{l}^{\frac{1}{2}}$, where $\boldsymbol{h}_{\mathrm{dl}, l}^{\mathrm{H}} \sim \mathcal{C N}\left(0, \boldsymbol{I}_{M}\right)$ and $\boldsymbol{R}_{l}$ is a 
spatial correlation matrix specific to terminal $l$, then

$$
\mathbb{E}\left[\left|\boldsymbol{g}_{\mathrm{d} l, l}^{\mathrm{H}} \boldsymbol{w}_{l}\right|^{2}\right]=M^{2}+\left\|\boldsymbol{R}_{l}\right\|_{\mathrm{F}}^{2}
$$

Proof of Lemma 7.4.1. One can begin by recognizing that

$$
\mathbb{E}\left[\left|\boldsymbol{g}_{\mathrm{dl}, l}^{\mathrm{H}} \boldsymbol{w}_{l}\right|^{2}\right]=\mathbb{E}\left[\left|\boldsymbol{g}_{\mathrm{dl}, l}^{\mathrm{H}} \boldsymbol{g}_{\mathrm{dl}, l}\right|^{2}\right]=\mathbb{E}\left[\left|\boldsymbol{h}_{\mathrm{dl}, l}^{\mathrm{H}} \boldsymbol{R}_{l} \boldsymbol{h}_{\mathrm{dl}, l}\right|^{2}\right]
$$

Invoking Lemma 2 of [168] and performing the expectation over the small-scale fading allows one to state

$$
\begin{aligned}
\mathbb{E}\left[\left|\boldsymbol{h}_{\mathrm{d} 1, l}^{\mathrm{H}} \boldsymbol{R}_{l} \boldsymbol{h}_{\mathrm{dl}, l}\right|^{2}\right] & =\left|\operatorname{tr}\left[\boldsymbol{I}_{M} \boldsymbol{R}_{l}\right]\right|^{2}+\operatorname{tr}\left[\boldsymbol{I}_{M} \boldsymbol{R}_{l}^{\mathrm{H}} \boldsymbol{I}_{M} \boldsymbol{R}_{l}\right] \\
& =M^{2}+\sum_{m=1}^{M} \sum_{\substack{n=1 \\
n \neq m}}^{M}\left|\boldsymbol{R}_{l ; m, n}\right|^{2} \stackrel{(a)}{=} M^{2}+\left\|\boldsymbol{R}_{l}\right\|_{\mathrm{F}}^{2},
\end{aligned}
$$

where $(a)$ is obtained by recognizing that $\left|\operatorname{tr}\left[\boldsymbol{I}_{M} \boldsymbol{R}_{l}\right]\right|^{2}=M^{2}$ and $\sum_{m=1}^{M} \sum_{\substack{n=1 \\ n \neq m}}^{M}\left|\left[\boldsymbol{R}_{l}\right]_{m, n}\right|^{2}=$ $\left\|\boldsymbol{R}_{l}\right\|_{\mathrm{F}}^{2}$, with $\left[\boldsymbol{R}_{l}\right]_{m, n}$ being the $(m, n)$-th entry of $\boldsymbol{R}_{l}$. This yields the desired result in (7.7).

Lemma 7.4.2. With $\boldsymbol{g}_{\mathrm{dl}, l}^{\mathrm{H}}=\boldsymbol{h}_{\mathrm{dl}, l}^{\mathrm{H}} \boldsymbol{R}_{l}^{\frac{1}{2}}$, the expected interference power for terminal $l$ in (7.6) is given by

$$
\sum_{\substack{k=1 \\ k \neq l}}^{L} \mathbb{E}\left[\left|\boldsymbol{g}_{\mathrm{dl}, l}^{\mathrm{H}} \boldsymbol{w}_{k}\right|^{2}\right]=(L-1)\left\{\operatorname{tr}\left[\boldsymbol{R}_{l} \overline{\boldsymbol{R}}_{-l}\right]\right\}
$$

where $\overline{\boldsymbol{R}}_{-l}=\frac{\sum_{k=1, k \neq l}^{L} \boldsymbol{R}_{l}}{L-1}$ is the average correlation matrix of all interfering terminals from $k=1, \ldots, L$ with $k \neq l$.

Proof of Lemma 7.4.2. With some algebraic manipulation, one can show that

$$
\mathbb{E}\left[\left|\boldsymbol{g}_{\mathrm{d} l, l}^{\mathrm{H}} \boldsymbol{w}_{k}\right|^{2}\right]=\mathbb{E}\left[\left|\boldsymbol{g}_{\mathrm{dl}, l}^{\mathrm{H}} \boldsymbol{g}_{\mathrm{dl}, k}\right|^{2}\right]=\mathbb{E}\left[\left|\boldsymbol{h}_{\mathrm{dl}, l}^{\mathrm{H}} \boldsymbol{Z} \boldsymbol{h}_{\mathrm{dl}, k}\right|^{2}\right]
$$


where $\boldsymbol{Z}=\boldsymbol{R}_{l}^{\frac{1}{2}} \boldsymbol{R}_{k}^{\frac{1}{2}}$ and

$$
\mathbb{E}\left[\left|\boldsymbol{h}_{\mathrm{dl}, l}^{\mathrm{H}} \boldsymbol{Z} \boldsymbol{h}_{\mathrm{dl}, k}\right|^{2}\right]=\mathbb{E}\left[\boldsymbol{h}_{\mathrm{dl}, l}^{\mathrm{H}} \boldsymbol{Z}^{\mathrm{H}} \boldsymbol{h}_{\mathrm{dl}, k} \boldsymbol{h}_{\mathrm{dl}, k}^{\mathrm{H}} \boldsymbol{Z} \boldsymbol{h}_{\mathrm{dl}, l}\right]
$$

Consider the eigen-decomposition of $\boldsymbol{Z}^{\mathrm{H}} \boldsymbol{h}_{\mathrm{dl}, k} \boldsymbol{h}_{\mathrm{dl}, k}^{\mathrm{H}} \boldsymbol{Z} \boldsymbol{Q} \boldsymbol{\Lambda} \boldsymbol{Q}^{\mathrm{H}}$, where $\boldsymbol{\Lambda}$ is the diagonal matrix of eigenvalues. It is noteworthy that $\boldsymbol{\Lambda}$ has a unit-rank, i.e., it only contains one non-zero eigenvalue, which is denoted as $\lambda_{1}$. From here, it is straightforward to show that $\lambda_{1}=\boldsymbol{h}_{\mathrm{dl}, k}^{\mathrm{H}} \boldsymbol{Z} \boldsymbol{Z}^{\mathrm{H}} \boldsymbol{h}_{\mathrm{dl}, k}$. Hence,

$$
\mathbb{E}\left[\boldsymbol{h}_{\mathrm{dl}, l}^{\mathrm{H}} \boldsymbol{Z}^{\mathrm{H}} \boldsymbol{h}_{\mathrm{dl}, k} \boldsymbol{h}_{\mathrm{dl}, k}^{\mathrm{H}} \boldsymbol{Z} \boldsymbol{h}_{\mathrm{dl}, l}\right] \stackrel{(a)}{=} \operatorname{tr}\left[\boldsymbol{R}_{k}^{\frac{1}{2}} \boldsymbol{R}_{l} \boldsymbol{R}_{k}^{\frac{1}{2}}\right] \stackrel{(b)}{=} \operatorname{tr}\left[\boldsymbol{R}_{l} \boldsymbol{R}_{k}\right],
$$

where $(a)$ is obtained by performing the expectation over $\boldsymbol{h}_{\mathrm{d} l, k}$ and recognizing that $\mathbb{E}\left[\boldsymbol{h}_{\mathrm{dl}, k} \boldsymbol{h}_{\mathrm{d} l, k}^{\mathrm{H}}\right]=\boldsymbol{I}_{M}$. Furthermore, (b) is obtained by applying the cyclic permutation property of the trace, allowing one to manipulate the order of the elements. Keeping this in mind, the total expected interference power at terminal $l$ in (7.6) is given by

$$
\begin{aligned}
\sum_{\substack{k=1 \\
k \neq l}}^{L} \mathbb{E}\left[\left|\boldsymbol{g}_{\mathrm{d}, l}^{\mathrm{H}} \boldsymbol{g}_{\mathrm{dl}, k}\right|^{2}\right] & =\sum_{\substack{k=1 \\
k \neq l}}^{L} \operatorname{tr}\left[\boldsymbol{R}_{l} \boldsymbol{R}_{k}\right]=\operatorname{tr}\left[\boldsymbol{R}_{l} \sum_{\substack{k=1 \\
k \neq l}}^{L} \boldsymbol{R}_{k}\right] \\
& =(L-1)\left\{\operatorname{tr}\left[\boldsymbol{R}_{l} \overline{\boldsymbol{R}}_{-l}\right]\right\} .
\end{aligned}
$$

This completes the proof.

Lemma 7.4.3. With $\boldsymbol{g}_{\mathrm{dl}, l}^{\mathrm{H}}=\boldsymbol{h}_{\mathrm{dl}, l}^{\mathrm{H}} \boldsymbol{R}_{l}^{\frac{1}{2}}$, the expected value of the normalization parameter, $\tilde{\eta}^{\mathrm{MF}}$, is given by

$$
\tilde{\eta}^{\mathrm{MF}}=\mathbb{E}\left[\eta^{\mathrm{MF}}\right]=M
$$

Proof of Lemma 7.4.3. The proof follows from the definition of $\eta^{\mathrm{MF}}$ 
allowing one to state

$$
\begin{aligned}
\tilde{\eta}^{\mathrm{MF}}=\frac{1}{L}\left\{\mathbb{E}\left[\operatorname{tr}\left[\boldsymbol{G}_{\mathrm{dl}} \boldsymbol{G}_{\mathrm{dl}}^{\mathrm{H}}\right]\right]\right\}= & \frac{1}{L}\left\{\mathbb{E}\left[\operatorname{tr}\left[\sum_{l=1}^{L} \boldsymbol{g}_{\mathrm{dl}, l} \boldsymbol{g}_{\mathrm{dl}, l}^{\mathrm{H}}\right]\right]\right\} \\
& =\frac{1}{L} \sum_{l=1}^{L} \mathbb{E}\left[\operatorname{tr}\left[\boldsymbol{g}_{\mathrm{dl}, l} \boldsymbol{g}_{\mathrm{d} l, l}^{\mathrm{H}}\right]\right] \\
& =\frac{1}{L} \sum_{l=1}^{L} \operatorname{tr}\left[\boldsymbol{R}_{l}\right]=M .
\end{aligned}
$$

This yields the desired result, leading to the following proposition.

Proposition 7.4.1 With MF precoding on the downlink, when $\boldsymbol{g}_{\mathrm{dl}, l}^{\mathrm{H}}=$ $\boldsymbol{h}_{\mathrm{dl}, l}^{\mathrm{H}} \boldsymbol{R}_{l}^{\frac{1}{2}}$ with $\boldsymbol{h}_{\mathrm{dl}, l}^{\mathrm{H}} \sim \mathcal{C N}\left(0, \boldsymbol{I}_{M}\right)$ and $\boldsymbol{R}_{l}$ being the correlation matrix specific to terminal $l$, the expected SINR for terminal $l$ in (7.6) can be approximated as

$$
\mathbb{E}\left[\mathrm{SINR}_{\mathrm{dl}, l}^{\mathrm{MF}}\right] \approx \frac{\rho_{\mathrm{dl}} \frac{\beta_{l}}{M}\left(M^{2}+\left\|\boldsymbol{R}_{l}\right\|_{\mathrm{F}}^{2}\right)}{\sigma_{\mathrm{dl}, l}^{2}+\rho_{\mathrm{dl}} \frac{\beta_{l}}{M}(L-1)\left\{\operatorname{tr}\left[\boldsymbol{R}_{l} \overline{\boldsymbol{R}}_{-l}\right]\right\}},
$$

where $\overline{\boldsymbol{R}}_{-l}$ is defined after and (7.10).

Method for Proposition 7.4.1. Substituting the results from Lemmas 7.4.1, 7.4.2 and 7.4.3 gives the desired expression in (7.17).

Remark 7.4.1. Proposition 7.4.1 presents a simple approximation to a complex situation where each terminal has a unique correlation matrix. With a fixed link gain, $\beta_{l}$, and a fixed noise power, $\sigma_{l}^{2}$, for terminal $l$, it is clear that both the numerator and the denominator of (7.17) are proportional to the number of service antennas, $M$, at the ULA, as well as the size of the spatial correlation matrix, $\boldsymbol{R}_{l}$, for terminal $l$. The denominator is also proportional to $L$, the total number of terminals served by the ULA. With all other parameters held constant, increasing $M$ increases the expected signal power quadratically, while increasing the expected interference power linearly, resulting in a net increase of order $M$ to the expected SINR for the $l$-th terminal. In contrast to this, fixing $M$ along with all other propagation parameters while increasing $L$ leads to a linear increase in the expected interference power proportional $L-1$. Moreover, fixing all other parameters, it can be observed that as $\operatorname{tr}\left[\boldsymbol{R}_{l} \overline{\boldsymbol{R}}_{-l}\right]$ increases, the total 
expected interference power at terminal $l$ also increases. As it directly influences the expected interference power, a more fundamental question is: When does $\operatorname{tr}\left[\boldsymbol{R}_{l} \overline{\boldsymbol{R}}_{-1}\right]$ reach its maximum value? To answer this question, the impact of variation in the correlation matrices on the expected MF SINR is considered. It is to be noted that the unequal correlation case results in $\boldsymbol{R}_{l} \neq \overline{\boldsymbol{R}}_{-l}$. In order to make a fair comparison with these distinct values of $\boldsymbol{R}_{l}$ and $\overline{\boldsymbol{R}}_{-l}$, for the equal correlation case, a common value given by $\frac{1}{2}\left(\boldsymbol{R}_{l}+\overline{\boldsymbol{R}}_{l}\right)$ is assumed for both correlation matrices. Therefore, the interference term relies on $\operatorname{tr}\left[\boldsymbol{R}_{l} \overline{\boldsymbol{R}}_{-l}\right]$ for the unequal correlation case and $\operatorname{tr}\left[\left(\frac{1}{2}\left(\boldsymbol{R}_{l}+\overline{\boldsymbol{R}}_{-l}\right)\right)^{2}\right]$ for the equal correlation case. Beginning with the fact that $\operatorname{tr}\left[\boldsymbol{A}^{2}\right] \geq 0$ for any Hermitian matrix $\boldsymbol{A}$, one can write ${ }^{11}$

$$
\begin{gathered}
\operatorname{tr}\left[\left(\frac{\boldsymbol{R}_{l}-\overline{\boldsymbol{R}}_{-l}}{2}\right)^{2}\right] \geq 0, \\
\operatorname{tr}\left[\frac{\boldsymbol{R}_{l}^{2}}{4}+\frac{\overline{\boldsymbol{R}}_{-l}^{2}}{4}-\frac{\boldsymbol{R}_{l} \overline{\boldsymbol{R}}_{-l}}{4}-\frac{\overline{\boldsymbol{R}}_{-l} \boldsymbol{R}_{l}}{4}\right] \geq 0, \\
\operatorname{tr}\left[\frac{\boldsymbol{R}_{l}^{2}}{4}+\frac{\overline{\boldsymbol{R}}_{-l}^{2}}{4}+\frac{1}{4} \boldsymbol{R}_{l} \overline{\boldsymbol{R}}_{-l}+\frac{1}{4} \overline{\boldsymbol{R}}_{-l} \boldsymbol{R}_{l}-\boldsymbol{R}_{l} \overline{\boldsymbol{R}}_{-l}\right] \geq 0, \\
\operatorname{tr}\left[\left(\frac{\boldsymbol{R}_{l}+\overline{\boldsymbol{R}}_{-l}}{2}\right)^{2}\right] \geq \operatorname{tr}\left[\boldsymbol{R}_{l} \overline{\boldsymbol{R}}_{-l}\right] .
\end{gathered}
$$

Hence, if $\boldsymbol{R}_{l}=\overline{\boldsymbol{R}}_{-l}$, the case for equal correlation matrices, terminal $l$ will have a higher total expected interference power in comparison to $\boldsymbol{R}_{l} \neq \overline{\boldsymbol{R}}_{-l}$, the case of unequal spatial correlation. Since this holds across all terminals, it can be concluded that conclude that equal correlation matrices result in the lowest SINR, assuming a fixed overall average correlation matrix. Therefore, the equal correlation matrices provide a useful lower bound for analysis of such systems.

Note that (7.17) can be further translated to approximate the ergodic sum

\footnotetext{
${ }^{11}$ Note that real eigenvalues are needed for this to hold, and thus the $\boldsymbol{A}$ has to be Hermitian. Since $\boldsymbol{R}_{1}, \ldots, \boldsymbol{R}_{L}$ are Hermitian matrices, it follows that $\overline{\boldsymbol{R}}_{-l}$ and $\boldsymbol{R}_{l} \overline{\boldsymbol{R}}_{-l}$ are also Hermitian.
} 
spectral efficiency of the system by

$$
\mathbb{E}\left[\mathrm{R}_{\mathrm{sum}, \mathrm{dl}}^{\mathrm{MF}}\right] \approx \sum_{l=1}^{L} \log _{2}\left(1+\mathbb{E}\left[\mathrm{SINR}_{\mathrm{dl}, l}^{\mathrm{MF}}\right]\right)
$$

Consistent with Chapters 3,4,5 and 6 of the thesis, it is also to be noted that (7.22) is an approximation to (7.5), as $\mathbb{E}\left[\mathrm{SINR}_{\mathrm{dl}, l}^{\mathrm{MF}}\right]$ itself is an approximation.

In the following subsection, an analysis with ZF precoding is carried out with unequal spatial correlation matrices.

\subsubsection{Expected ZF SNR and Ergodic Sum Spectral Efficiency}

The denominator of the ZF SNR for terminal $l$ in (7.4) contains $\operatorname{tr}\left[\left(\boldsymbol{G}_{\mathrm{dl}}^{\mathrm{H}} \boldsymbol{G}_{\mathrm{dl}}\right)^{-1}\right]$. Finding the exact moments of this term is an extremely challenging task, except for the simplest case of uncorrelated i.i.d. Rayleigh fading [140]. Hence, we approximate the inverse in (7.4) by a finite Neumann series (NS) expansion, as demonstrated in Chapter $4[77,78] .{ }^{12}$ To do this, $\boldsymbol{G}_{\mathrm{dl}}^{\mathrm{H}} \boldsymbol{G}_{\mathrm{dl}}$ is separated into its expected diagonal component and correction terms, such that

$$
\boldsymbol{G}_{\mathrm{dl}}^{\mathrm{H}} \boldsymbol{G}_{\mathrm{dl}}=M \boldsymbol{I}_{L}+\boldsymbol{\Delta},
$$

where

$$
\boldsymbol{\Delta}=\boldsymbol{G}_{\mathrm{dl}}^{\mathrm{H}} \boldsymbol{G}_{\mathrm{dl}}-M \boldsymbol{I}_{L},
$$

and $\mathbb{E}[\boldsymbol{\Delta}]=\mathbf{0}$. As a result, with an order $N$ NS, one can approximate the inverse of $\boldsymbol{G}_{\mathrm{dl}}^{\mathrm{H}} \boldsymbol{G}_{\mathrm{dl}}$ as

$$
\left(\boldsymbol{G}_{\mathrm{dl}}^{\mathrm{H}} \boldsymbol{G}_{\mathrm{dl}}\right)^{-1} \approx \frac{1}{M} \sum_{p=0}^{N}(-1)^{p}\left(\frac{\boldsymbol{\Delta}}{M}\right)^{p}
$$

\footnotetext{
${ }^{12}$ This approach was mentioned briefly in Chapter 4, where the effects of unequal correlation matrices were first analyzed.
} 
Substituting the definition of $\boldsymbol{\Delta}$ yields

$$
\left(\boldsymbol{G}_{\mathrm{dl}}^{\mathrm{H}} \boldsymbol{G}_{\mathrm{dl}}\right)^{-1} \approx \frac{1}{M} \sum_{p=0}^{N} \frac{(-1)^{p}}{(M)^{p}}\left(\boldsymbol{G}_{\mathrm{dl}}^{\mathrm{H}} \boldsymbol{G}_{\mathrm{dl}}-M \boldsymbol{I}_{L}\right)^{p}
$$

Further expansion and simplification results in

$$
\begin{aligned}
\left(\boldsymbol{G}_{\mathrm{dl}}^{\mathrm{H}} \boldsymbol{G}_{\mathrm{dl}}\right)^{-1} & \approx \frac{1}{M} \sum_{p=0}^{N} \frac{(-1)^{p}}{(M)^{p}} \sum_{q=0}^{p}\left(\begin{array}{l}
p \\
q
\end{array}\right)\left(\boldsymbol{G}_{\mathrm{dl}}^{\mathrm{H}} \boldsymbol{G}_{\mathrm{dl}}\right)^{q}(-M)^{p-q} \\
& =\frac{1}{M} \sum_{p=0}^{N} \sum_{q=0}^{p}\left(\begin{array}{l}
p \\
q
\end{array}\right) \frac{(-1)^{q}}{(M)^{q}}\left(\boldsymbol{G}_{\mathrm{dl}}^{\mathrm{H}} \boldsymbol{G}_{\mathrm{dl}}\right)^{q}
\end{aligned}
$$

Substituting (7.27) allows us to approximate $\mathrm{SNR}_{\mathrm{dl}, l}^{\mathrm{ZF}}$ in (7.4) as

$$
\mathrm{SNR}_{\mathrm{dl}, l}^{\mathrm{ZF}} \approx \frac{\rho_{\mathrm{dl}} \beta_{l}}{\sigma_{\mathrm{dl}, l}^{2}\left\{\operatorname{tr}\left[\frac{1}{M} \sum_{p=0}^{N} \sum_{q=0}^{p}\left(\begin{array}{l}
p \\
q
\end{array}\right) \frac{(-1)^{q}}{(M)^{q}}\left(\boldsymbol{G}_{\mathrm{dl}}^{\mathrm{H}} \boldsymbol{G}_{\mathrm{dl}}\right)^{q}\right]\right\}}
$$

To evaluate (7.28), the univariate special case of the first-order delta expansion motivated in Chapter 3, Section 3.4.3 is employed. After some algebraic manipulation, one can express the expected ZF SNR for the $l$-th terminal as

$$
\mathbb{E}\left[\mathrm{SNR}_{\mathrm{dl}, l]}^{\mathrm{ZF}}\right] \approx \frac{\rho_{\mathrm{dl}} \beta_{l}}{\sigma_{\mathrm{dl}, l}^{2}\left\{\frac{1}{M} \sum_{p=0}^{N} \sum_{q=0}^{p}\left(\begin{array}{l}
p \\
q
\end{array}\right) \frac{(-1)^{q}}{(M)^{q}} \mathbb{E}\left[\operatorname{tr}\left[\left(\boldsymbol{G}_{\mathrm{dl}}^{\mathrm{H}} \boldsymbol{G}_{\mathrm{dl}}\right)^{q}\right]\right]\right\}}
$$

In the following proposition, with a two-term NS (i.e., $N=2$ ), a closed-form solution to (7.29) is presented for the general case where each terminal has an unequal spatial correlation matrix.

Proposition 7.4.2. When $\boldsymbol{g}_{\mathrm{dl}, l}^{\mathrm{H}}=\boldsymbol{h}_{\mathrm{dl}, l}^{\mathrm{H}} \boldsymbol{R}_{l}^{\frac{1}{2}}$, where $\boldsymbol{h}_{\mathrm{dl}, l}^{\mathrm{H}} \sim \mathcal{C N}\left(0, \boldsymbol{I}_{M}\right)$ and $\boldsymbol{R}_{l}$ is a terminal specific transmit spatial correlation matrix, the expected perterminal SNR at the $l$-th terminal with ZF precoding in (7.4) can be approximated by

$$
\mathbb{E}\left[\mathrm{SNR}_{\mathrm{dl}, l]}^{\mathrm{ZF}}\right] \approx \frac{\rho_{\mathrm{dl}} \beta_{l} M^{3}}{\sigma_{\mathrm{d} l, l}^{2}\left\{L\left(M^{2}+L\left\{\operatorname{tr}\left[\overline{\boldsymbol{R}}^{2}\right]\right\}\right)\right\}}
$$

where $\overline{\boldsymbol{R}}=\frac{\sum_{j=1}^{L} \boldsymbol{R}_{j}}{L}$ is the average correlation matrix of all terminals in the 
system. ${ }^{13}$

Method for Proposition 7.4.2. From (7.28), when $N=2$, one can write

$$
\begin{aligned}
\left(\boldsymbol{G}_{\mathrm{dl}}^{\mathrm{H}} \boldsymbol{G}_{\mathrm{dl}}\right)^{-1} & \approx \frac{1}{M}\left[\boldsymbol{I}_{L}-\frac{\boldsymbol{G}_{\mathrm{dl}}^{\mathrm{H}} \boldsymbol{G}_{\mathrm{dl}}}{M}+\boldsymbol{I}_{L}+\frac{\left(\boldsymbol{G}_{\mathrm{dl}}^{\mathrm{H}} \boldsymbol{G}_{\mathrm{dl}}\right)^{2}}{(M)^{2}}-\frac{2}{M} \boldsymbol{G}_{\mathrm{dl}}^{\mathrm{H}} \boldsymbol{G}_{\mathrm{dl}}+\boldsymbol{I}_{L}\right] \\
& =\frac{1}{M}\left[3 \boldsymbol{I}_{L}-\frac{3}{M} \boldsymbol{G}_{\mathrm{dl}}^{\mathrm{H}} \boldsymbol{G}_{\mathrm{dl}}+\frac{1}{M^{2}}\left(\boldsymbol{G}_{\mathrm{dl}}^{\mathrm{H}} \boldsymbol{G}_{\mathrm{dl}}\right)^{2}\right] .
\end{aligned}
$$

Taking the trace of (7.31) yields

$$
\operatorname{tr}\left[\left(\boldsymbol{G}_{\mathrm{dl}}^{\mathrm{H}} \boldsymbol{G}_{\mathrm{dl}}\right)^{-1}\right] \approx \frac{1}{M}\left\{3 L-\frac{3}{M} \operatorname{tr}\left[\boldsymbol{G}^{\mathrm{H}} \boldsymbol{G}\right]+\frac{1}{M^{2}} \operatorname{tr}\left[\left(\boldsymbol{G}_{\mathrm{dl}}^{\mathrm{H}} \boldsymbol{G}_{\mathrm{dl}}\right)^{2}\right]\right\} .
$$

The subtraction of the first two terms on the right-hand side of (7.32) results in a constant (discussed prior to (7.35)). This allows one to primarily focus on the trace in the final term of $(7.32)$, which can be re-written as

$$
\operatorname{tr}\left[\left(\boldsymbol{G}_{\mathrm{dl}}^{\mathrm{H}} \boldsymbol{G}_{\mathrm{dl}}\right)^{2}\right]=\sum_{i=1}^{L} \sum_{j=1}^{L} \boldsymbol{g}_{\mathrm{dl}, i}^{\mathrm{H}} \boldsymbol{g}_{\mathrm{dl}, j} \boldsymbol{g}_{\mathrm{dl}, j}^{\mathrm{H}} \boldsymbol{g}_{\mathrm{dl}, i}
$$

Performing the expectation of (7.33) over the fast-fading yields

$$
\begin{aligned}
\mathbb{E}\left[\operatorname{tr}\left[\left(\boldsymbol{G}_{\mathrm{dl}}^{\mathrm{H}} \boldsymbol{G}_{\mathrm{dl}}\right)^{2}\right]\right] & =\mathbb{E}\left[\sum_{i=1}^{L}\left(\boldsymbol{g}_{\mathrm{dl}, i}^{\mathrm{H}} \boldsymbol{g}_{\mathrm{dl}, i}\right)^{2}+\sum_{i=1}^{L} \sum_{\substack{j=1 \\
j \neq i}}^{L} \boldsymbol{g}_{\mathrm{dl}, i}^{\mathrm{H}} \boldsymbol{g}_{\mathrm{dl}, j} \boldsymbol{g}_{\mathrm{dl}, j}^{\mathrm{H}} \boldsymbol{g}_{\mathrm{dl}, i}\right] \\
& =\mathbb{E}\left[\sum_{i=1}^{L}\left\{M^{2}+\operatorname{tr}\left[\boldsymbol{R}_{i}^{2}\right]\right\}+\sum_{i=1}^{L} \sum_{\substack{j=1 \\
j \neq i}}^{L} \operatorname{tr}\left[\boldsymbol{R}_{i} \boldsymbol{R}_{j}\right]\right] \\
& =L M^{2}+\sum_{i=1}^{L} \sum_{j=1}^{L} \operatorname{tr}\left[\boldsymbol{R}_{i} \boldsymbol{R}_{j}\right] \\
& =L M^{2}+\operatorname{tr}\left[\sum_{i=1}^{L} \boldsymbol{R}_{i} \sum_{j=1}^{L} \boldsymbol{R}_{j}\right] \\
& =L\left(M^{2}+L\left\{\operatorname{tr}\left[\overline{\boldsymbol{R}}^{2}\right]\right\}\right) .
\end{aligned}
$$

${ }^{13}$ This is in contrast with the expected MF SINR result in (7.17), whose denominator composed of $\operatorname{tr}\left[\boldsymbol{R}_{l} \overline{\boldsymbol{R}}_{-l}\right]$, where $\overline{\boldsymbol{R}}_{-l}$ was the average correlation matrix of all interfering terminals, instead of all terminals in the system. 
Substituting (7.34) into (7.32) and simplifying using the fact that $\mathbb{E}\left[\operatorname{tr}\left[\left(\boldsymbol{G}_{\mathrm{dl}}^{\mathrm{H}} \boldsymbol{G}_{\mathrm{dl}}\right)\right]\right]=$ $M L$, yields

$$
\mathbb{E}\left[\operatorname{tr}\left[\left(\boldsymbol{G}_{\mathrm{dl}}^{\mathrm{H}} \boldsymbol{G}_{\mathrm{dl}}\right)^{-1}\right]\right] \approx \frac{1}{M^{3}} L\left\{M^{2}+L \operatorname{tr}\left[\overline{\boldsymbol{R}}^{2}\right]\right\}
$$

where $\overline{\boldsymbol{R}}$ is defined in (7.30). Substituting (7.35) into the denominator of (7.29) and performing some routine algebra yields the desired expression. This concludes the proof.

Remark 7.4.2. The result in Proposition 7.4.2 also provides a closed-form solution to a complex scenario, where each terminal experiences unequal levels of spatial correlation. The remarkably simple structure of (7.30) readily demonstrates the impact of unequal correlation matrices, through the $\operatorname{tr}\left[\overline{\boldsymbol{R}}^{2}\right]$ term in the denominator of (7.30). Unlike the MF case, with the global power normalization of the ZF precoding matrix, the expected SNR is the same for each terminal, with the exception of the link gain, $\beta_{l}$, which is dependent on terminal l. Holding all other propagation and system parameters fixed and increasing the number of serving antennas, $M$, will indefinitely increase the expected SINR at the rate of order $M$ due to the numerator of the expected SINR containing a $M^{3}$ and the denominator containing a $M^{2}$ term. In contrast to this, holding all other parameters constant and increasing $L$ without bound will lead to an increase in the expected noise power proportional to $L^{2}$. Such insights are missing at large from more complex solutions derived in the literature (see e.g., $[43,44,60,61,116]$ and the references therein) which require a linked set of equations, even in the large system regime, where $M$ and/or $L$ can be assumed to be pushed to infinity.

Remark 7.4.3. The generality of the results presented in Propositions 7.4.1 and 7.4.2 is worth highlighting. The results are applicable for any system dimension, link SNR level and are capable of handling any type of spatial correlation structure. The derived results can also be applied to conventional multicellular systems, distributed antenna arrays, as well as small-cellular systems, where the additional effects of inter cellular interference can be characterized in the 
exact same way. ${ }^{14}$ In addition to this, the ergodic sum spectral efficiency of the system with ZF precoding can be approximated in the same manner as for MF precoding, such that

$$
\mathbb{E}\left[\mathrm{R}_{\mathrm{sum}, \mathrm{dl}}^{\mathrm{ZF}}\right] \approx \sum_{l=1}^{L} \log _{2}\left(1+\mathbb{E}\left[\mathrm{SNR}_{\mathrm{dl}, l}^{\mathrm{ZF}}\right]\right)
$$

Numerical evaluation of the derived results in Propositions 7.4.1 and 7.4.2, along with their respective ergodic sum spectral efficiency approximations is presented in the subsequent section.

\subsection{Numerical Results}

Unless otherwise specified, parameters for the numerical evaluation are as follows: A circular cell with a radius of $100 \mathrm{~m}$ is considered, with terminals which are uniformly distributed over the cell coverage area. A reference distance of $d_{0}=10 \mathrm{~m}$ is assumed with the unit-less geometric attenuation constant, $v$, where $v$ is chosen for the subsequent numerical results such that the instantaneous SINR with processing is $0 \mathrm{~dB}, 95 \%$ of the time. The attenuation exponent and shadow-fading standard deviation are $\alpha=3.7$ and $\sigma_{\mathrm{sf}}=8 \mathrm{~dB}$, respectively [15]. The noise power for each terminal is set to $\sigma_{\mathrm{dl}}^{2}=1$ and for each subsequent result, $10^{4}$ Monte-Carlo trails were carried out. An inter-element spacing of $0.5 \lambda$ is assumed between adjacent elements in the ULA. Prior to presenting the numerical results, details for the spatial correlation models under investigation is presented.

\subsubsection{Spatial Correlation Models}

Considering a ULA, as a baseline case, equal correlation for each terminal is modeled with the widely used non-physical exponential correlation model, where the correlation matrix for terminal $l$ is given by $\left[\boldsymbol{R}_{l}\right]_{i, j}=\varphi^{|i-j|}, \forall l=1, \ldots, L$

\footnotetext{
${ }^{14}$ This is merely pointed out to demonstrate the true generality of the derived results, hence, no numerical evaluation of the performance of such systems is presented.
} 
for $i, j \in 1, \ldots, M[46]$ with $0 \leq \varphi \leq 1$. The advantage of this model lies in its simplicity, as it is controlled by a single-parameter, $\varphi$, common to each terminal. On the other hand, when each terminal is assigned an unequal correlation matrix, three models in the Clerckx [120], one-ring (O.R.) $[43,117]$ and Laplacian (Lap.) $[14,117]$ are employed. While the former is non-physical, the two latter models are in the category of remote scattering models and are physically motivated by channel measurements [14]. For the Clerckx correlation model, terminal l's correlation matrix is given by $\left[\boldsymbol{R}_{l}\right]_{i, j}=\varphi_{c}^{|i-j|}$, where $\rho_{c}=|\varphi| e^{j \phi_{l}}$. Here, $|\varphi|=\varphi$ in the exponential model and is the same for each terminal, as it captures the effects of inter-element spacing at the ULA. Moreover, a unique phase $\phi_{l} \sim \mathcal{U}[a, b]$, a subset of $[0,2 \pi]$ is used to differentiate the terminal locations in the azimuth domain. In the subsequent figures, the Clerckx model is refereed to as C. Corr and the exponential model is refereed to as Exp. Corr., respectively. In contrast to the above, the O.R. model with a ULA for terminal $l$ states

$$
\left[\boldsymbol{R}_{l}\right]_{i, j}=\frac{1}{2 \Delta} \int_{-\Delta+\phi_{0}^{l}}^{\Delta+\phi_{0}^{l}} e^{-j 2 \pi d_{s}(i, j) \sin \left(\phi_{l}\right)} d \phi_{l}
$$

where $\Delta$ denotes the angular spread in the azimuth domain, $\phi_{0}^{l}$ denotes the central azimuth angle seen from the serving array, $\phi_{l}$ is the actual DoA and $d_{s}(i, j)$ captures the inter-element spacing normalized by the carrier wavelength between the $i$-th and $j$-th antenna elements. Note that the O.R. model assumes a uniform distribution of received power from planar waves impinging on the serving antennas. Alternatively, modeling the correlation with a Laplacian power azimuth spectrum results in

$$
\left[\boldsymbol{R}_{l}\right]_{i, j}=\frac{1}{\gamma} \int_{\phi_{0}^{l}-\pi}^{\phi_{0}^{l}+\pi} e^{-\frac{\sqrt{2}}{\theta}\left|\phi_{l}-\phi_{0}^{l}\right|-j 2 \pi d_{s}(i, j) \sin \left(\phi_{l}\right)} d \phi_{l}
$$

where $\gamma=1 / \sqrt{2} \theta\left(1-e^{\frac{-\sqrt{2} \pi}{\theta}}\right)$ is the normalization constant [169]. For a fair comparison between the O.R. and Lap. correlation models, both the models were ensured to have the same root mean square (rms) angular spread, denoted 
as $\sigma_{\phi^{l}}$. Hence, for the O.R. model with a uniform power azimuth spectrum, $\Delta=\sqrt{3} \sigma_{\phi^{l}}$ and for the Lap. case, $\theta$ is the numerical solution to

$$
\sigma_{\phi}^{l}=\sqrt{\gamma\left[\frac{-\pi^{2} \delta}{\sqrt{2} / \theta}-\frac{2 \pi \delta}{(\sqrt{2} / \theta)^{2}}-\frac{2 \delta}{(\sqrt{2} / \theta)^{3}}+\frac{2}{(\sqrt{2} / \theta)^{3}}\right]},
$$

respectively, where $\delta=e^{-\frac{\sqrt{2}}{\theta}} \pi[169]$.

In the following subsection, numerical results for the analytical approximations derived in (7.17), (7.22), (7.30) and (7.36) with above mentioned correlation structures are evaluated for a MU-MIMO systen.

\subsubsection{Performance of Unequal vs. Equal Spatial Correlation}

Fig. 7.2 illustrates the expected per-terminal SINR with ZF precoding as a function of the link SNR with $M=8$ and $L=3$. In addition to the exponential (baseline) and Clerckx correlation models, the uncorrelated i.i.d. Rayleigh fading case is also considered as an upper bound for performance comparison purposes.

Three trends can be observed from the figure: Firstly, with unequal correlation matrices from the Clerckx model, the larger the spread of the random phases in $\phi_{l}$, the higher the expected SINRs. In spite of the correlation magnitude being as high as $\varphi=0.99$, increasing the spread of $\phi_{l}$ to $\mathcal{U}[0, \pi]$ and $\mathcal{U}[0,2 \pi]$ in comparison to fixing $\phi_{l}$ (equal correlation) yields a 6 and $9 \mathrm{~dB}$ gain respectively in the expected SINR at any given link SNR level. ${ }^{15}$ This tremendous performance difference is owed due to the fact that increasing the spread of $\phi_{l}$ increases the amount of spatial diversity induced between the channels, allowing the composite channel rank to increase. This results in an increase in the spatially usable degrees of freedom giving the superior performance. Such a significant performance difference demonstrates the sensitivity of MU-MIMO

\footnotetext{
${ }^{15}$ Note that a slight discrepancy can be observed in the numerical results, where the symbol " $u[a, b]$ " is used to signify $\mathcal{U}[a, b]$, a uniform distribution on $[a, b]$.
} 


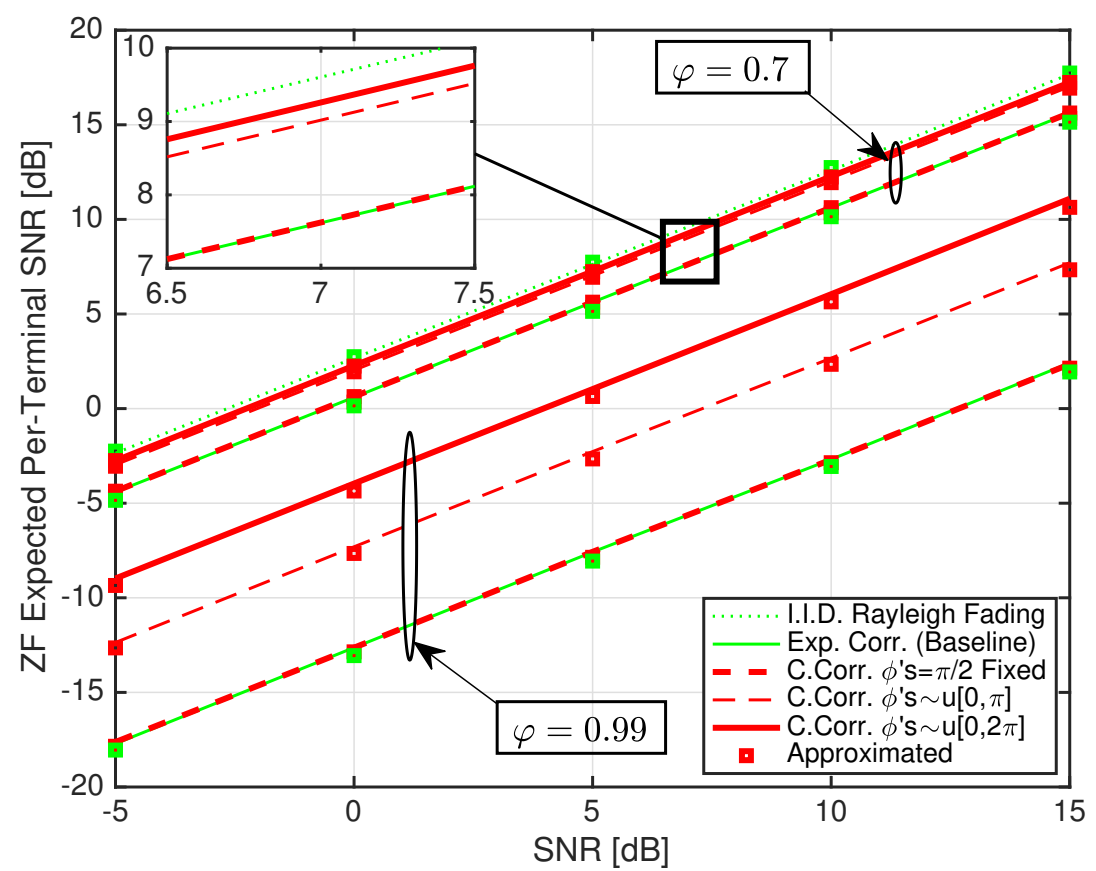

Figure 7.2: Expected ZF SNR vs. link SNR with $M=8$ and $L=3$.

systems to changes in the phase of the correlation matrices between terminals. On the other hand, fixing $\phi_{l}^{\prime} s$ to a particular value diminishes the diversity of the phases in the correlation matrices, resulting in equal (exponential) correlation structures, yielding lower expected SINRs. Secondly, decreasing $\varphi$ from 0.99 to 0.7 also provides a large enhancement in the expected SINRs both with equal (exponential) and unequal (Clerckx) correlation matrices. A decrease in the level of correlation causes higher diversity to be leveraged in the channel, leading to lower noise noise enhancement in the ZF precoder. As the correlation magnitude is relatively small, one can observe a lesser difference between the uncorrelated i.i.d. and correlated cases. Thirdly, the derived approximations are sufficiently accurate for the entire range of link SNR for all cases considered. The above discussion is in agreement with Remarks 7.4 .1 and 7.4.2, where it was analytically predicted that equal correlation structures would result in lower performance in comparison to unequal correlation.

Fig. 7.3 demonstrates the expected MF SINR vs. link SNR for the same system parameters. Here, similar trends as in Fig. 7.2 can be observed for both 


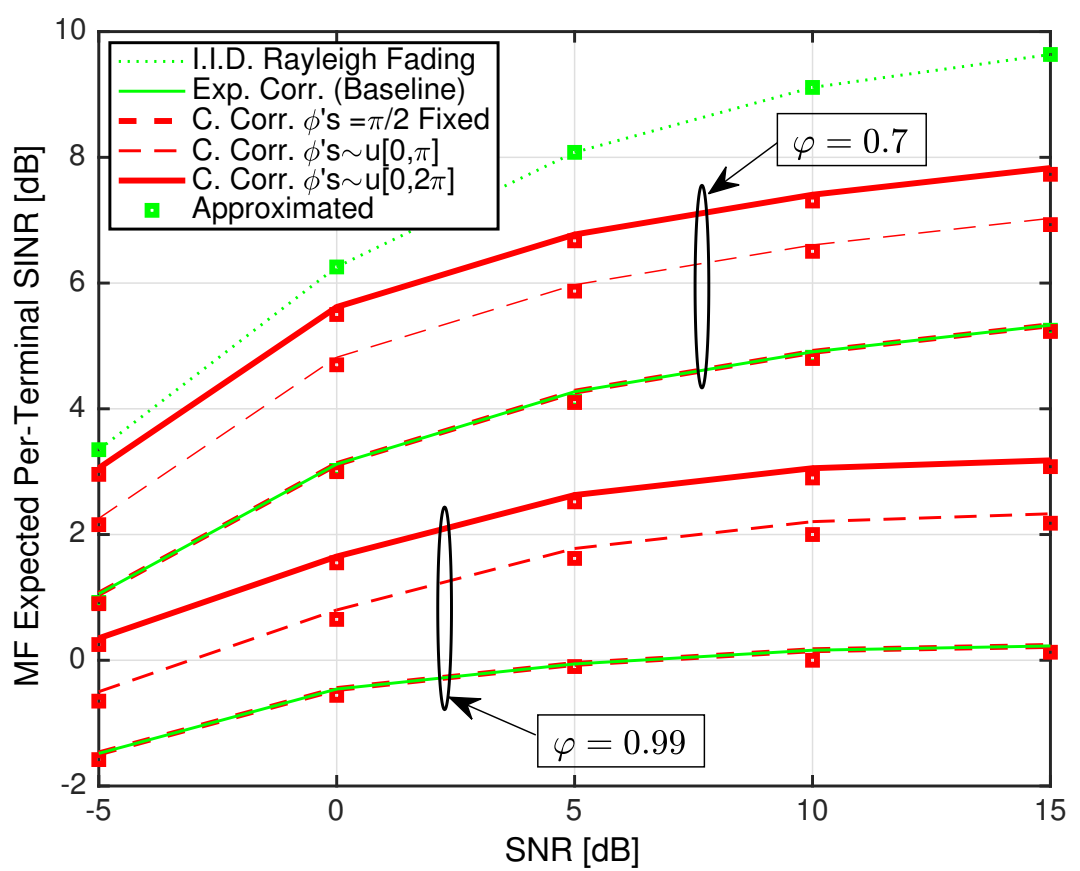

Figure 7.3: Expected MF SINR vs. link SNR with $M=8$ and $L=3$.

$\varphi=0.7$ and 0.99. It is noteworthy that for both $\varphi=0.7$ and $\varphi=0.99$, the gain obtained from increasing the spread of $\varphi_{l}$ (unequal correlation matrices) from $\mathcal{U}[0, \pi]$ to $\mathcal{U}[0,2 \pi]$ is not as prominent as in the ZF case. This is due to the MF precoder's inability to mitigate the presence of multiuser interference. Thus, the expected SINRs are seen to reach a plateau at higher link SNRs $(>10$ $\mathrm{dB})$. For lower values of link SNRs $(<0 \mathrm{~dB})$, the performance of MF precoding gives significantly better performance due to its ability to maximize the desired signal power whilst being insusceptible to the strong receiver noise presence at such low SNRs. The derived expressions for the expected SINR with increasing link SNRs are observed to be tight.

Now the influence of increasing the number of serving antennas with a fixed number of terminals is investigated on the ergodic sum spectral efficiency with unequal and equal correlation matrices, considering MF and ZF precoding. Fig. 7.4 depicts the ergodic sum spectral efficiency as a function of link SNR. Increasing $M$ from 8 to 30 (transitioning from top to the bottom subfigure) naturally increases the ergodic sum spectral efficiency for both the exponential 


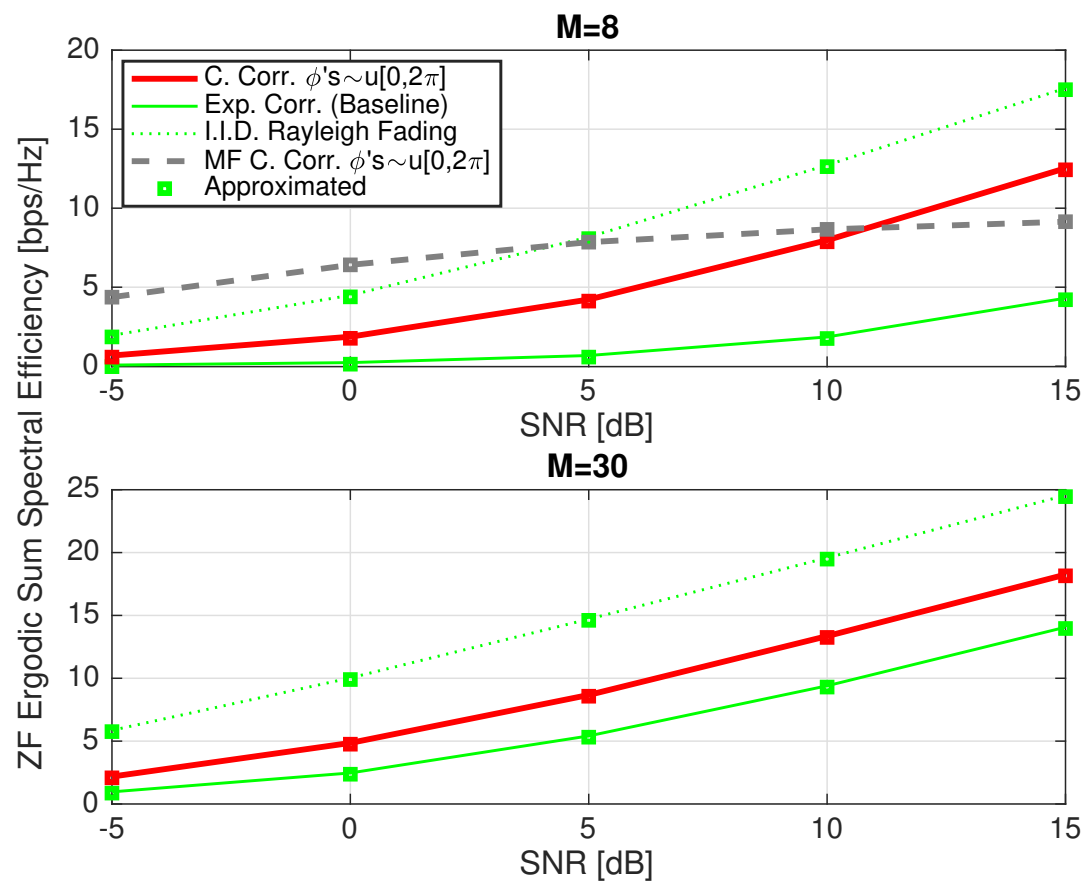

Figure 7.4: ZF (and MF where explicitly stated) ergodic sum spectral efficiency vs. link SNR with $M=8,30$ and $L=3$.

(equal) and Clerckx (unequal) correlation models. When $M=8$, the ergodic sum spectral efficiency with MF precoding saturates (in the top subfigure) due to the remaining interference and crosses its equivalent ZF counterpart at a link SNR level of $11 \mathrm{~dB}$. For both $M=8$ and 30 (i.e., in both the subfigures), it can be readily seen that the derived approximations remain tight over the range of link SNRs considered, consistent with Remark 7.4.3.

The expected ZF SNR as a function of the link SNR is now compared with O.R. and Lap. unequal correlation models for $M=8$ and $K=3$. Fig. 7.5 demonstrates two trends: First, with a wide rms angular spread, $\sigma_{\phi_{l}}=70^{\circ}$, performance of both the O.R. and Lap. correlation models is equivalent to the uncorrelated i.i.d. Rayleigh case. This is owed to the increased spatial diversity in the propagation channel due to the larger angular spreads. As a further consequence of this, one can also observe that the expected SNR is insensitive to changes in the terminal's central azimuth angle, where a marginal change in the expected ZF SNR can be observed when serving each terminal broadside to the ULA. Nevertheless, the O.R. model with its uniform power azimuth spectrum 


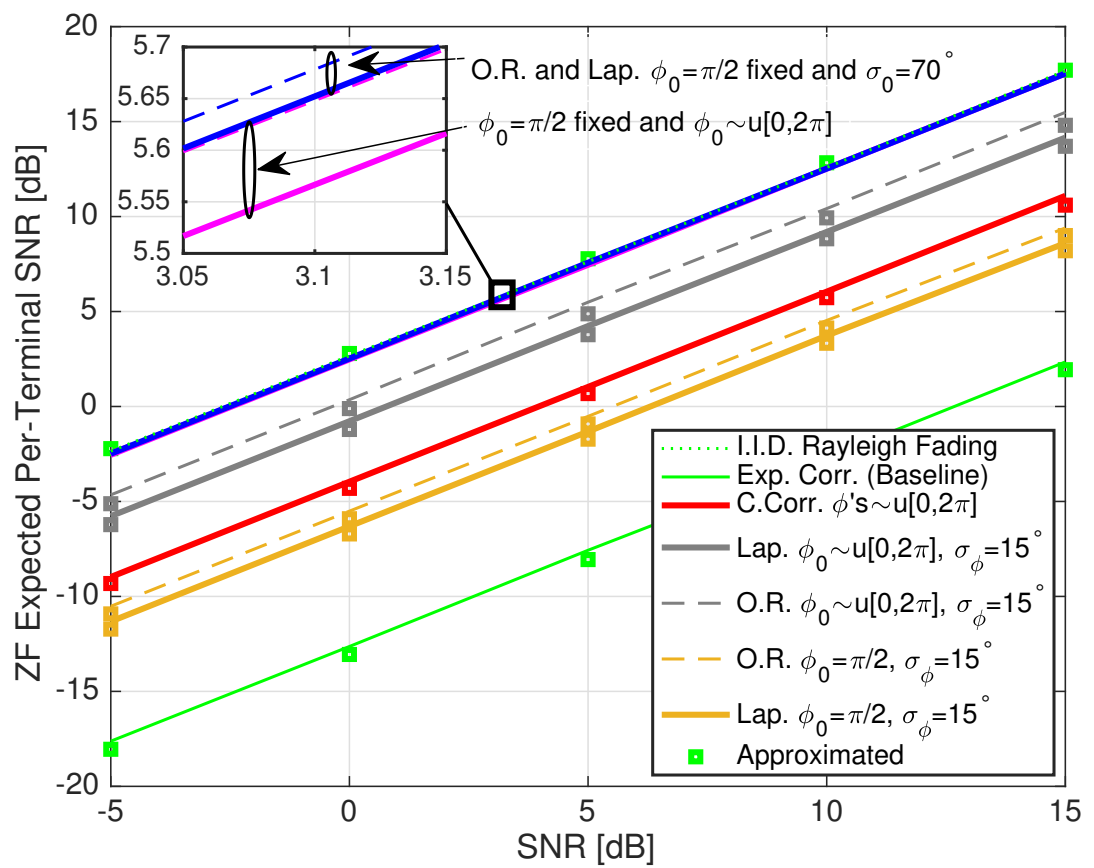

Figure 7.5: Expected ZF SNR vs. link SNR with $M=8$ and $L=3$.

still outperforms the Lap. model, which has a narrow peak in its power azimuth spectrum. Secondly, for narrow rms angular spreads, the gap between the O.R. and Lap. models widens, where the expected ZF SNR with the O.R. model is higher than the Lap. case with both fixed and variable central azimuth angles. Here, the expected SNR is sensitive to the variability in the central azimuth angles. We observe an expected SNR difference of approximately $5 \mathrm{~dB}$ going from fixed to variable central DoA. Moreover, for smaller rms angular spreads, both O.R. and Lap. give higher expected SINRs than the Clerckx correlation model. This may be due to the fact that in addition to the unique phase components, the correlation magnitudes in the O.R. and Lap. models are also unique for each terminal. As a result, their combined effect induces an even higher degree of spatial diversity, further improving the per-terminal performance. Once again one can observe that equal (exponential) correlation matrices yield a much lower performance and thus is an effective baseline performance measure, as shown in Remarks 7.4.1 and 7.4.2.

In Fig. 7.6, a similar trend to Fig. 7.3 can be observed for MF precoding 


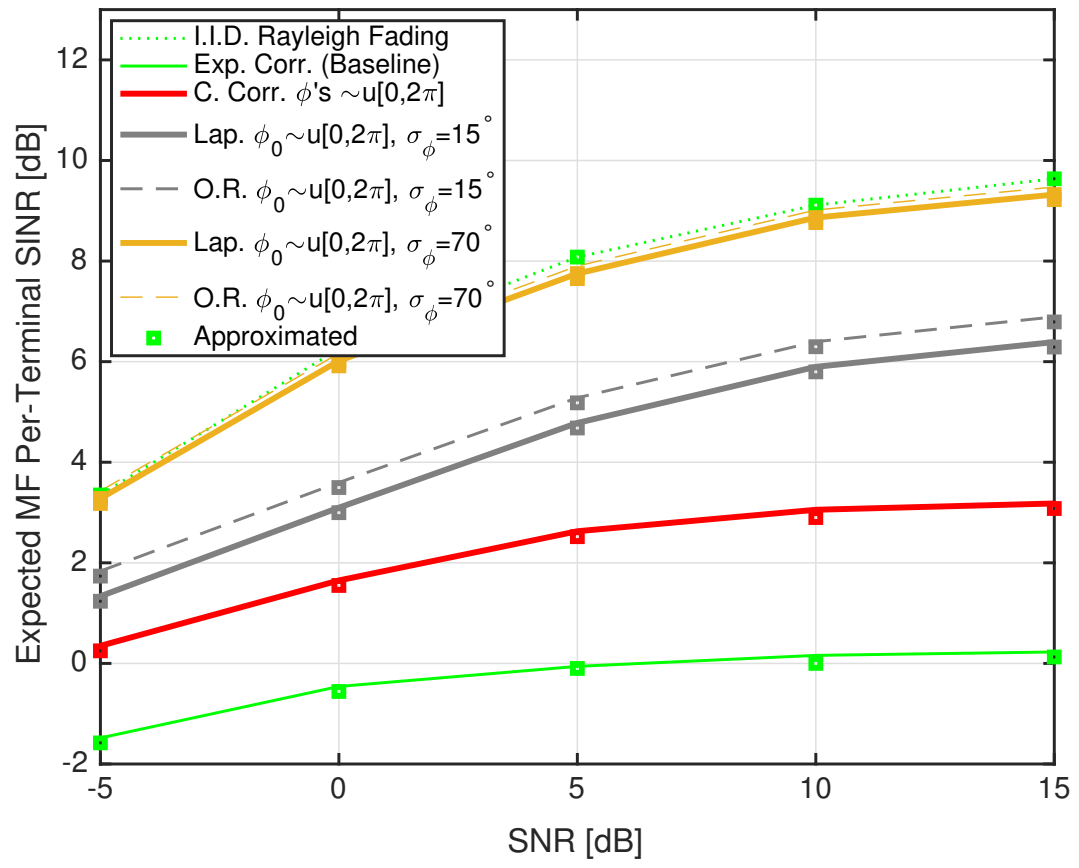

Figure 7.6: Expected MF SINR vs. link SNR with $M=8$ and $L=3$.

where the O.R. model is again seen to give higher SINRs than the Lap. for both narrow and wide rms angular spreads. Both models give higher SINRs than the Clerckx model, whilst all simulated and analytical responses are seen to saturate due to the large presence of interference at high link SNRs. In Fig. 7.7, with $M=30$ and $K=3$, the ergodic sum spectral efficiency of both MF and ZF precoders was evaluated for narrow rms angular spreads. It can be seen that at low SNRs, MF performance dominates ZF, while at link SNR $>12 \mathrm{~dB}$, ZF outperforms MF. In both Figs. 7.7 and 7.6, the analytical expressions are again seen to retain their accuracy.

\subsection{Conclusion}

This chapter presented closed-form approximations of expected per-terminal SINR, expected per-terminal SNR and ergodic sum spectral efficiency of a MUMIMO system with MF and ZF precoding. Considering spatially correlated Rayleigh fading with unequal correlation matrices, the analysis is robust to changes in the system size, link SNR levels and various physical and non-physical 


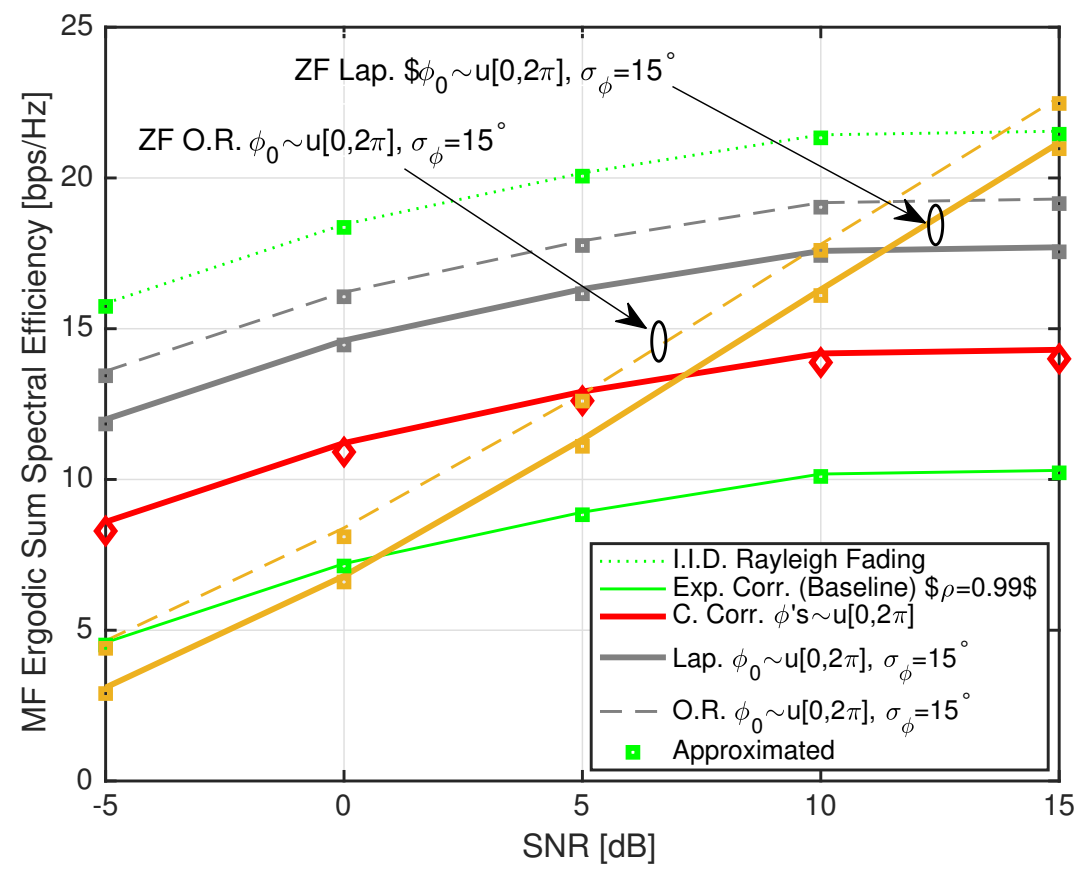

Figure 7.7: MF (and ZF where explicitly stated) ergodic sum spectral efficiency vs. link SNR with $M=30$ and $L=3$.

correlation models. With both MF and ZF precoding, it is found that the total expected interference and noise terms tend to increase with equal correlation matrices for each terminal, resulting in smaller expected SINR and SNR, respectively. More physically motivated remote scattering models such as O.R. and Lap., which consider unique correlation magnitudes and phases per-terminal tend to predict higher performance than the exponential and Clerckx correlation models, which consider fixed correlation magnitudes for each terminal. To the best of the author's knowledge, such a performance analysis and evaluation of a MU-MIMO system is unique and emphasizes that fact that the performance of the system is ultimately governed by the respective spatial correlation model in use. Moreover, it also emphasizes the fact that unique levels of scattering around each terminal contributes to greater spatial diversity in the channel. Such an important detail is missing from models which design a fixed correlation matrix for each terminal, yet is present in remote scattering models which design unique correlation matrices for each terminal. 


\section{Chapter 8}

\section{Uplink MRC Analysis With Fixed Physical Array Sizes}

Summary. The unprecedented spectral and energy efficiency gains on offer with large antenna arrays are commonly based on the existence of favorable propagation. That is, as the number of service antennas at a cellular base station (BS) grows without bound, propagation vectors to and from two distinct terminals become mutually orthogonal, removing multiuser interference. In contrast to many prior studies which consider fixed inter-element spacing between two adjacent antennas, and therefore an increasing array aperture with growing number of elements, fixed physical array sizes are considered in this chapter. Here, an increase in the number of antennas in a fixed space imposes an inversely proportional decrease in the inter-element spacing. More specifically, the chapter analyzes the uplink performance of large multiuser multiple-input multiple-output systems, where the BS antennas are configured in a uniform linear array (ULA) with a fixed total space. An investigation into the combined effects of two contradicting phenomena is carried out: (1) the reduction of spatial diversity due to reducing the spatial separation between the BS elements, and (2) the increase in receive diversity by increasing the sheer number of elements. In this context, with maximum-ratio combining (MRC) at the BS, the chapter presents a general analytical framework for approximating the ergodic sum spectral efficiency, by approximating the expected (average) per-terminal 
signal-to-interference-plus-noise-ratio (SINR). Furthermore, the chapter extends and generalizes a prior physical space-constrained (SC) channel model, by including the effects of unequal levels of receive spatial correlation and unequal levels of line-of-sight (LoS) for each terminal. In addition to the above, deterministic limits of the SINR and sum spectral efficiency are also analyzed when the number of service antennas is increased indefinitely with a fixed number of terminals. The derived analytical expressions lead to numerous useful insights into the behavior of the expected SINR and ergodic sum spectral efficiency with variations in the system and propagation parameters. Supporting numerical results demonstrate that a SC ULA deployment with MRC processing causes saturation of the expected SINR and ergodic sum spectral efficiency with increasing link signal-to-noise-ratio (SNR) and serving antennas. Several special cases of the derived expressions are also presented when only non LoS components are present with equal and unequal spatial correlation matrices, as well as when each terminal having LoS components has equal correlation matrices.

\subsection{Introduction}

The emergence of large-scale antenna arrays has posed new engineering challenges, which must be overcome before its rapid expansion on a scale commensurate with its true potential. One of the critical issues is accommodating a large number of antenna elements in constrained physical spaces at the BS $[9,57,89,90,170]$. This tends to increase the level of spatial correlation and antenna coupling, as successive elements are placed in close proximity with interelement spacings less than the desired half-a-wavelength $[81,84,89,156,171]$. In the multiuser context, this is known to cause a detrimental impact on the terminal signal-to-interference-plus-noise-ratio (SINR) and system spectral efficiency, as the radio channel is unable to provide sufficient decorrelation between the different closely spaced antennas. It is thus important to rigorously analyze and evaluate the performance of large multiuser multiple-input multiple-output (MU-MIMO) systems with space-constrained (SC) antenna arrays. 
With the knowledge of channel state information at both the transmitter and the receiver, a number of works have investigated the impact of SC antenna arrays on the performance of large MU-MIMO systems. For instance, the effects of fitting a large number of antennas in size-constrained structures have been studied in [59, 89-93, 159]. More specifically, [89] analyzed the ergodic sum spectral efficiency of large MU-MIMO systems with fixed array dimensions. The authors of [90] demonstrated that multiuser interference does not vanish in large SC MU-MIMO systems with growing numbers of antennas. The uplink performance with maximum-ratio combining (MRC), zero-forcing and minimummean-squared-error receivers has been analyzed in $[59,93]$ where the authors derive upper and lower bounds on the ergodic sum spectral efficiency. Moreover, $[91,92,159]$ investigated the energy efficiency performance of SC systems with various large-scale antenna array topologies considering antenna coupling.

However, almost none of the above mentioned studies consider the effects of line-of-sight (LoS) components ${ }^{1}$, which may be a dominant feature in future wireless systems with the rise of smaller cell sizes, potentially operating in the millimeter-wave (mmWave) frequency bands [80,109]. Hence, understanding the performance of large MU-MIMO systems with SC arrays and LoS presence, i.e., with Ricean fading is of particular importance [82]. It is also worth noting that the respective channel models in $[59,93,159]$ assume that all terminals are seen by the BS array via the same set of incident directions, resulting in equal spatial correlation structures for each terminal. However, in reality, as each terminal is located in a unique geographical location, differences in local scattering around each terminal can give rise to wide variations in the correlation patterns across their local terrain [40]. In addition to the smaller inter-element spacings, this further influences the level of correlation in the channel, impacting the terminal SINR and system spectral efficiency. Therefore, to more accurately capture the correlation and LoS differences in multiple user channels, this chapter considers unequal correlation matrices and unequal LoS levels, unique to each terminal.

\footnotetext{
${ }^{1}$ An exception is made for [90], which considers pure LoS channels. This is an extreme case, which in general may not be realizable in practice even at mmWave frequencies, where at least 1-3 scattering clusters are anticipated on average in the propagation channel [137].
} 
Motivated by the aforementioned considerations, with a SC uniform linear array (ULA), the chapter presents a general analytical framework for the analysis of expected per-terminal SINR and ergodic sum spectral efficiency of large uplink MU-MIMO systems with MRC receiver processing at the BS. ${ }^{2}$

More specifically, the notable contributions of this chapter are listed as follows:

1. The performance of uplink MU-MIMO systems with SC ULAs under spatially correlated Ricean fading is analyzed. In doing so, the chapter extends and generalizes the physical space-constrained channel model presented in $[59,89-93,159]$ to cater for unequal correlation matrices and LoS levels with an unequal Rice factor for each terminal. To the best of the authors' knowledge, such generality in the channel model has not previously been considered.

2. With MRC at the BS array, tight closed-form approximations to the expected per-terminal SINR and ergodic sum spectral efficiency are derived. It is observed that the SC antenna deployment causes a saturation of the expected SINR, as the number of BS antennas and link signal-tonoise-ratio (SNR) is increased. The derived expressions lend themselves to important insights into the behavior of the expected SINR and ergodic sum spectral efficiency as a function of various system and propagation parameters.

3. With a fixed number of user terminals, as the number of antenna elements in the ULA increases without bound in a fixed physical space, novel closed-form expressions are derived to analyze the deterministic limits of the expected SINR and ergodic sum spectral efficiency. The limiting expressions demonstrate the convergence behavior of large SC MU-MIMO systems and emphasize their inability to leverage favorable propagation conditions.

\footnotetext{
${ }^{2}$ Though not shown, the analytical approach presented in the chapter can be extended to other SC arrays, such as the uniform rectangular and cylindrical arrays.
} 
4. In addition to the above, special cases of the derived analytical expressions are presented when considering both LoS and NLoS with equal correlation, and additionally considering NLoS with unequal correlation, respectively.

The rest of the chapter is structured as follows: Section 8.2 details the SC system model, performance metrics of interest and presents the proposed channel model for SC ULAs. Section 8.3 analyzes the expected per-terminal SINR and ergodic sum spectral efficiency with spatially correlated Ricean fading channels. Section 8.4 presents the special cases of the expected SINR approximation derived in Section 8.3, under the conditions of equal and unequal spatial correlation structures in Rayleigh fading channels, as well as for Ricean fading channels having equal correlation structures for all terminals. Section 8.5 analyzes the deterministic limits of the expected SINR and ergodic sum spectral efficiency, as the number of receive antennas at the ULA are increased indefinitely with a fixed number of terminals. Section 8.6 presents the numerical performance evaluation of the derived approximations, while Section 8.7 presents the concluding remarks of the chapter.

\subsection{System Model}

\subsubsection{Signal Model}

The uplink of a large MU-MIMO system operating in an urban microcellular (UMi) environment is considered. Here, $L$ non-cooperative single-antenna user terminals transmit data to $M$ receive antennas at the $\mathrm{BS}(M \gg L)$ in the same time-frequency interval. The BS comprises of a ULA with equispaced, omnidirectional antennas. No mechanical downtilt is assumed at the BS array. Channel knowledge at the BS is assumed with narrow-band transmission and no uplink power control. The composite $M \times 1$ received signal at the BS array can be written as

$$
\boldsymbol{y}_{\mathrm{ul}}=\rho_{\mathrm{ul}}^{\frac{1}{2}} \boldsymbol{G}_{\mathrm{ul}} \boldsymbol{D}^{\frac{1}{2}} \boldsymbol{s}_{\mathrm{ul}}+\boldsymbol{n}_{\mathrm{ul}}
$$


where $\rho_{\mathrm{ul}}$ is the average transmit power of each terminal, $\boldsymbol{G}_{\mathrm{ul}}$ denotes the normalized $M \times L$ fast-fading uplink channel matrix between $M$ BS antennas and $L$ terminals (discussed further in Section 8.2.2), $\boldsymbol{D}$ is an $L \times L$ diagonal matrix of link gains for the $L$ terminals in the system, such that $[\boldsymbol{D}]_{l, l}=\beta_{l}$. The large-scale fading effects for terminal $l$ are captured

$$
\beta_{l}=v\left(\frac{d_{0}}{d_{l}}\right)^{\alpha} \zeta_{l}
$$

In particular, $v$ denotes the unit-less constant for geometric attenuation at a reference distance $d_{0}, d_{l}$ denotes the link distance between the BS array and terminal $l, \alpha$ denotes the attenuation exponent and $\zeta_{l}$ models the effects of shadow-fading following a log-normal density, i.e., $10 \log _{10}\left(\zeta_{l}\right) \sim \mathcal{N}\left(0, \sigma_{\text {sf }}^{2}\right)$, with $\sigma_{\text {sf }}$ denoting the shadow-fading standard deviation. ${ }^{3}$ Numerical values for the above are tabulated in Section 8.6. The $L \times 1$ vector of uplink data symbols from the $L$ terminals is given by $\boldsymbol{s}_{\mathrm{ul}}$, such that the $l$-th entry of $\boldsymbol{s}_{\mathrm{ul}}, s_{l}$ has $\mathbb{E}\left[\left|s_{\mathrm{ul}, l}\right|^{2}\right]=$ 1. Additive white Gaussian noise entries at the $M$ BS antennas are given by the $M \times 1$ vector $\boldsymbol{n}_{\mathrm{ul}}$, such that the $m$-th entry of $\boldsymbol{n}_{\mathrm{ul}}, n_{\mathrm{ul}, m} \sim \mathcal{C N}\left(0, \sigma_{\mathrm{ul}, m}^{2}\right)$. For the remainder of the chapter, it is assumed that $\sigma_{\mathrm{ul}}^{2}=\sigma_{\mathrm{ul}, m}^{2}=1$, for all $m=1, \ldots, M$. Hence, the link SNR is defined as $\rho_{\mathrm{ul}} / \sigma_{\mathrm{ul}}^{2}=\rho_{\mathrm{ul}}$.

\subsubsection{Proposed Space-Constrained Channel Model}

Previous studies (see e.g., $[59,93,159])$ on large SC MU-MIMO systems consider a physical channel model based on full NLoS propagation conditions, where the BS sees the same set of scattered directions from each terminal. Such a model is extended here to cater for the presence of LoS in the propagation channel, as well as a unique set of scattered directions from each terminal taking into account differences in the local scattering around each terminal. Specifically, $\boldsymbol{G}_{\mathrm{ul}}=\left[\boldsymbol{g}_{\mathrm{ul}, 1}, \ldots, \boldsymbol{g}_{\mathrm{ul}, L}\right]$, where $\boldsymbol{g}_{\mathrm{ul}, l}$, the $l$-th column of $\boldsymbol{G}_{\mathrm{ul}}$ contains the $M \times 1$

\footnotetext{
${ }^{3}$ Note that the classical large-scale fading model is employed here from the earlier chapters in the thesis. As the uplink of a MU-MIMO system is considered, the received signal in (8.1) is scaled by the average uplink transmit power, assumed equal for each terminal. Due to this reason, the expression in (8.2) is not scaled by the average uplink transmit power.
} 
uplink channel vector from terminal $l$ to the BS array given by

$$
\boldsymbol{g}_{\mathrm{ul}, l}=\left(\sqrt{\frac{1}{1+K_{l}}} \frac{1}{\sqrt{P}}\right) \boldsymbol{A}_{l} \boldsymbol{h}_{\mathrm{ul}, l}+\sqrt{\frac{K_{l}}{K_{l}+1}} \overline{\boldsymbol{h}}_{\mathrm{ul}, l}
$$

In the above, $\sqrt{1 /\left(1+K_{l}\right)}$ and $\sqrt{K_{l} /\left(K_{l}+1\right)}$ balance the amount of power present in the diffuse and specular components of the channel according to the Ricean $K$-factor, $K_{l}$, specific to terminal $l[14]$. Moreover, $\sqrt{1 /\left(1+K_{l}\right)}$ is further scaled by a factor of $\frac{1}{\sqrt{P}}$ to normalize the steering vectors in $\boldsymbol{A}_{l}$, the $M \times P$ transmit steering matrix associated with the diffuse components of the channel. Here, $P$ denotes a large yet finite number of diffuse wavefronts. For ULAs

$$
\boldsymbol{A}_{l}=\left[\boldsymbol{a}\left(\phi_{l, 1}\right), \boldsymbol{a}\left(\phi_{l, 2}\right), \ldots, \boldsymbol{a}\left(\phi_{l, P}\right)\right]
$$

where each vector in (8.4) is given by

$$
\boldsymbol{a}\left(\phi_{l, i}\right)=\left[1, e^{j 2 \pi d \sin \left(\phi_{l, i}\right)}, \ldots, e^{j 2 \pi(M-1) d \sin \left(\phi_{l, i}\right)}\right]
$$

Note that $i \in 1, \ldots, P$, with $d$ denoting the equidistant inter-element spacing normalized by the carrier wavelength, $\lambda_{c} ; \phi_{l, i} \in[-\Delta / 2, \Delta / 2]$ denotes the $i$ th direction-of-arrival (DOA) from terminal $l$ to the BS array and $\Delta$ is the angular spread in the azimuth domain. With such a model, different degrees of receive correlations can be modeled by varying the angular spread. Moreover, $\boldsymbol{h}_{\mathrm{ul}, l} \sim \mathcal{C N}\left(0, \boldsymbol{I}_{P}\right)$ is the $P \times 1$ vector of diffuse channel gains, whilst $\overline{\boldsymbol{h}}_{\mathrm{ul}, l}$ is the $M \times 1$ vector denoting the specular component of the channel and is governed by the ULA's steering response with a LoS DoA, $\bar{\phi}_{l}$ for terminal $l$, such that

$$
\overline{\boldsymbol{h}}_{\mathrm{ul}, l}=\left[1, e^{j 2 \pi d \sin \left(\bar{\phi}_{l}\right)}, \ldots, e^{j 2 \pi(M-1) d \sin \left(\bar{\phi}_{l}\right)}\right]
$$

Remark 8.2.1. For both $\boldsymbol{a}\left(\phi_{l, i}\right)$ and $\overline{\boldsymbol{h}}_{l}$, it is to be noted that the normalized total array length, $\bar{d}_{0}$, is fixed at the BS, such that the inter-element spacing between two successive antenna elements is given by $d=\frac{\bar{d}_{0}}{M-1} \lambda_{\mathrm{c}}$. Since the physical dimensions of the BS array are predetermined, the above model ac- 
curately allows us to capture the correlation due to close proximity of adjacent antenna elements positioned at the array. This, along with the unique correlation matrices for each terminal created by the $\boldsymbol{A}_{l}$ for $l \in 1, \ldots, L$, constitutes our focus in the following sections.

Remark 8.2.2. Furthermore, it is to be noted that when two successive elements receiving (or transmitting) electromagnetic waves are placed in extremely close proximity, some of the energy which is primarily intended for one particular element is seen at other elements $[91,92,172]$. More specifically, the electric and the magnetic field of a particular element impacts the current distribution of the adjacent elements, which leads to perturbation in the radiation pattern and input impedance of each antenna element [173]. This is known as mutual coupling. Indeed, one would expect the performance of such systems to be influenced by this physical artifact. However, it has been shown in [174] (and quoted in [91]) that the effects of mutual coupling can be reduced significantly with impedance matching approaches. In line with these previous studies, the performance of space-constrained arrays is studied without considering the effects of mutual coupling.

To determine the level of LoS and NLoS present in the simulated propagation channel from a given terminal to the BS, a probability based approach is employed in line with $[81,84]$. Both LoS and NLoS probabilities are a function of the link distance, from which the LoS and NLoS geometric attenuation, as well as other link characteristics are obtained. Propagation parameters from both microwave [136] and mmWave [137] frequency bands are considered. For notational clarity, the discussion of the above mentioned parameters is deferred to Section 8.6.

\subsubsection{SINR and Ergodic Sum Spectral Efficiency}

As linear signal processing techniques perform near optimally for large MUMIMO systems $[9,57,74]$, a linear receiver in the form of a MRC is considered at the BS. The $L \times M$ MRC matrix, $\boldsymbol{T}=\boldsymbol{G}_{\mathrm{ul}}^{\mathrm{H}}$, is used to separate $\boldsymbol{y}_{\mathrm{ul}}$ into $L$ 
streams by ${ }^{4}$

$$
\boldsymbol{r}_{\mathrm{ul}}=\boldsymbol{G}_{\mathrm{ul}}^{\mathrm{H}} \boldsymbol{y}_{\mathrm{ul}}=\rho_{\mathrm{ul}}^{\frac{1}{2}} \boldsymbol{G}_{\mathrm{ul}}^{\mathrm{H}} \boldsymbol{G}_{\mathrm{ul}} \boldsymbol{D}^{\frac{1}{2}} \boldsymbol{s}_{\mathrm{ul}}+\boldsymbol{G}_{\mathrm{ul}}^{\mathrm{H}} \boldsymbol{n}_{\mathrm{ul}} \cdot
$$

Thus, the linearly combined signal from terminal $l$ is given by

$$
r_{\mathrm{ul}, l}=\rho_{\mathrm{ul}}^{\frac{1}{2}} \beta_{l}^{\frac{1}{2}} \boldsymbol{g}_{\mathrm{ul}, l}^{\mathrm{H}} \boldsymbol{g}_{\mathrm{ul}, l} s_{\mathrm{ul}, l}+\rho_{\mathrm{ul}}^{\frac{1}{2}} \sum_{\substack{k=1 \\ k \neq l}}^{L} \beta_{k}^{\frac{1}{2}} \boldsymbol{g}_{\mathrm{ul}, l}^{\mathrm{H}} \boldsymbol{g}_{\mathrm{ul}, k} s_{\mathrm{ul}, k}+\boldsymbol{g}_{\mathrm{ul}, l}^{\mathrm{H}} \boldsymbol{n}_{\mathrm{ul}},
$$

resulting in the corresponding SINR given by

$$
\mathrm{SINR}_{\mathrm{ul}, l}=\frac{\rho_{\mathrm{ul}} \beta_{l}\left\|\boldsymbol{g}_{\mathrm{ul}, l}\right\|^{4}}{\left\|\boldsymbol{g}_{\mathrm{ul}, l}\right\|^{2}+\rho_{\mathrm{ul}} \sum_{\substack{k=1 \\ k \neq l}}^{L} \beta_{k}\left|\boldsymbol{g}_{\mathrm{ul}, l}^{\mathrm{H}} \boldsymbol{g}_{\mathrm{ul}, k}\right|^{2}}
$$

Hence, the instantaneous achievable uplink spectral efficiency for terminal $l$ (measured in bits/sec/Hz) can be computed as $\mathrm{R}_{\mathrm{ul}, l}=\log _{2}\left(1+\mathrm{SINR}_{\mathrm{ul}, l}\right)$. As such, the ergodic sum spectral efficiency over all $L$ terminals is given by

$$
\mathbb{E}\left[\mathrm{R}_{\text {sum }, \mathrm{ul}}\right]=\mathbb{E}\left[\sum_{l=1}^{L} \mathrm{R}_{\mathrm{ul}, l}\right]
$$

where the expectation is performed over the small-scale fading. In the following section, tight analytical expressions are derived to approximate the expected value of (8.9) and (8.10), respectively.

\subsection{Expected SINR and Ergodic Sum Spectral Efficiency Analysis}

As motivated in Chapter 3, Section 3.4.3, the expected SINR for terminal $l$ can be obtained by taking the expectation of the ratio in (8.9). This gives

\footnotetext{
${ }^{4}$ For ease of exposition, the key system equations are repeated here.
} 


$$
\mathbb{E}\left[\mathrm{SINR}_{\mathrm{ul}, l}\right] \approx \frac{\rho_{\mathrm{ul}} \beta_{l} \mathbb{E}\left[\|\left.\boldsymbol{g}_{\mathrm{ul}, l}\right|^{4}\right]}{\mathbb{E}\left[\|\left.\boldsymbol{g}_{\mathrm{ul}, l}\right|^{2}\right]+\rho_{\mathrm{ul}} \sum_{\substack{k=1 \\ k \neq l}}^{L} \beta_{k} \mathbb{E}\left[\left|\boldsymbol{g}_{\mathrm{ul}, l}^{\mathrm{H}} \boldsymbol{g}_{\mathrm{ul}, k}\right|^{2}\right]}
$$

In the sequel, Lemmas 8.3.1, 8.3.2 and 8.2.3 derive the expectations in the numerator and denominator of (8.11). For the ease of reference, the remainder of the chapter denotes $\varsigma_{l}=\sqrt{\frac{1}{1+K_{l}}}, \overline{\varsigma_{l}}=\sqrt{\frac{K_{l}}{K_{l}+1}}$ and $\varsigma_{l}^{\prime}=\varsigma_{l} \frac{1}{\sqrt{P}}$.

Lemma 8.3.1. For a ULA with $M$ receive antennas in a fixed physical space at the BS, considering a correlated Ricean fading uplink channel, $\boldsymbol{g}_{\mathrm{ul}, l}$, from terminal $l$ to the BS array

$$
\begin{aligned}
\delta_{l}=\mathbb{E}\left[\left\|\boldsymbol{g}_{\mathrm{ul}, l}\right\|^{4}\right]= & \left(\varsigma_{l}^{\prime}\right)^{4}\left\{P^{2} M^{2}+\operatorname{tr}\left[\left(\boldsymbol{A}_{l}^{\mathrm{H}} \boldsymbol{A}_{l}\right)^{2}\right]\right\}+2 P M^{2}\left(\varsigma_{l}^{\prime}\right)^{2}\left(\bar{\varsigma}_{l}\right)^{2} \\
& +2\left(\varsigma_{l}^{\prime}\right)^{2}\left(\bar{\varsigma}_{l}\right)^{2} \overline{\boldsymbol{h}}_{\mathrm{ul}, l}^{\mathrm{H}} \boldsymbol{A}_{l} \boldsymbol{A}_{l}^{\mathrm{H}} \overline{\boldsymbol{h}}_{\mathrm{ul}, l}+\left(\bar{\varsigma}_{l}\right)^{4} M^{2} .
\end{aligned}
$$

Proof of Lemma 8.3.1. Via its definition, $\delta_{l}=\mathbb{E}\left[\| \boldsymbol{g}_{\mathrm{ul}, l}||^{4}\right]=\mathbb{E}\left[\left(\left\|\boldsymbol{g}_{\mathrm{ul}, l}\right\|^{2}\right)^{2}\right]$. Furthermore, substituting the definition of $\boldsymbol{g}_{\mathrm{ul}, l}$ and denoting $\boldsymbol{v}_{l}=\varsigma_{l}^{\prime} \boldsymbol{A}_{l} \boldsymbol{h}_{\mathrm{ul}, l}$ and $\boldsymbol{q}_{l}=\bar{\varsigma}_{l} \overline{\boldsymbol{h}}_{\mathrm{ul}, l}$ allows us to state

$$
\delta_{l}=\mathbb{E}\left[\left(\left\|\boldsymbol{g}_{\mathrm{ul}, l}\right\|^{2}\right)^{2}\right]=\mathbb{E}\left[\left(\boldsymbol{v}_{l}^{\mathrm{H}} \boldsymbol{v}_{l}+\boldsymbol{v}_{l}^{\mathrm{H}} \boldsymbol{q}_{l}+\boldsymbol{q}_{l}^{\mathrm{H}} \boldsymbol{v}_{l}+\boldsymbol{q}_{l}^{\mathrm{H}} \boldsymbol{q}_{l}\right)^{2}\right]
$$

Expanding (8.13) and simplifying allows us to state

$$
\begin{aligned}
\delta_{l}=\mathbb{E}\left[\left(\left\|\boldsymbol{g}_{\mathrm{ul}, l}\right\|^{2}\right)^{2}\right] & =\mathbb{E}\left[\left(\boldsymbol{v}_{l}^{\mathrm{H}} \boldsymbol{v}_{l}\right)^{2}\right]+\mathbb{E}\left[2\left(\boldsymbol{v}_{l}^{\mathrm{H}} \boldsymbol{v}_{l}\right)\left(\boldsymbol{q}_{l}^{\mathrm{H}} \boldsymbol{q}_{l}\right)\right]+\mathbb{E}\left[\boldsymbol{v}_{l}^{\mathrm{H}} \boldsymbol{q}_{l} \boldsymbol{q}_{l}^{\mathrm{H}} \boldsymbol{v}_{l}\right] \\
& +\mathbb{E}\left[\boldsymbol{q}_{l}^{\mathrm{H}} \boldsymbol{v}_{l} \boldsymbol{v}_{l}^{\mathrm{H}} \boldsymbol{q}_{l}\right]+\mathbb{E}\left[\left(\boldsymbol{q}_{l}^{\mathrm{H}} \boldsymbol{q}_{l}\right)^{2}\right] .
\end{aligned}
$$

Furthermore,

$$
\begin{aligned}
\mathbb{E}\left[\boldsymbol{v}_{l}^{\mathrm{H}} \boldsymbol{v}_{l}\right] \stackrel{(a)}{=} \mathbb{E}\left[\varsigma_{l}^{\prime} \boldsymbol{h}_{\mathrm{ul}, l}^{\mathrm{H}} \boldsymbol{A}_{l}^{\mathrm{H}} \varsigma_{l}^{\prime} \boldsymbol{A}_{l} \boldsymbol{h}_{\mathrm{ul}, l}\right] \\
=\left(\varsigma_{l}^{\prime}\right)^{2} \mathbb{E}\left[\boldsymbol{h}_{\mathrm{ul}, l}^{\mathrm{H}} \boldsymbol{A}_{l}^{\mathrm{H}} \boldsymbol{A}_{l} \boldsymbol{h}_{\mathrm{ul}, l}\right] \\
=\left(\varsigma_{l}^{\prime}\right)^{2} \mathbb{E}\left[\operatorname{tr}\left[\boldsymbol{h}_{\mathrm{ul}, l}^{\mathrm{H}} \boldsymbol{A}_{l}^{\mathrm{H}} \boldsymbol{A}_{l} \boldsymbol{h}_{\mathrm{ul}, l}\right]\right] \\
\stackrel{(b)}{=}\left(\varsigma_{l}^{\prime}\right)^{2} \operatorname{tr}\left[\boldsymbol{A}_{l}^{\mathrm{H}} \boldsymbol{A}_{l}\right]=\left(\varsigma_{l}^{\prime}\right)^{2} M P,
\end{aligned}
$$


where (a) is obtained by substituting the definition of $\boldsymbol{v}_{l}$ and (b) is obtained by performing the expectation over $\boldsymbol{h}_{\mathrm{ul}, l}$. This allows one to express (8.14) as

$$
\delta_{l}=\mathbb{E}\left[\left(\left\|\boldsymbol{g}_{\mathrm{ul}, l}\right\|^{2}\right)^{2}\right]=\mathbb{E}\left[\left(\boldsymbol{v}_{l}^{\mathrm{H}} \boldsymbol{v}_{l}\right)^{2}\right]+2\left(\boldsymbol{q}_{l}^{\mathrm{H}} \boldsymbol{q}_{l}\right)\left(\varsigma_{l}^{\prime}\right)^{2} P M+2\left(\varsigma_{l}^{\prime}\right)^{2} \boldsymbol{q}_{l}^{\mathrm{H}} \boldsymbol{A}_{l} \boldsymbol{A}_{l}^{\mathrm{H}} \boldsymbol{q}_{l}+\left(\boldsymbol{q}_{l}^{\mathrm{H}} \boldsymbol{q}_{l} \boldsymbol{q}_{l}^{\mathrm{H}} \boldsymbol{q}_{l}\right)
$$

Note that the expectation in the last four terms of (8.14) is performed over $\boldsymbol{h}_{\mathrm{ul}, l}$. Now as $\mathbb{E}\left[\left(\boldsymbol{v}_{l}^{\mathrm{H}} \boldsymbol{v}_{l}\right)^{2}\right]=\mathbb{E}\left[\boldsymbol{v}_{l}^{\mathrm{H}} \boldsymbol{v}_{l} \boldsymbol{v}_{l}^{\mathrm{H}} \boldsymbol{v}_{l}\right]$, substituting the definition of $\boldsymbol{v}_{l}$ and extracting the relevant constants yields

$$
\mathbb{E}\left[\boldsymbol{v}_{l}^{\mathrm{H}} \boldsymbol{v}_{l} \boldsymbol{v}_{l}^{\mathrm{H}} \boldsymbol{v}_{l}\right]=\left(\varsigma_{l}^{\prime}\right)^{4} \mathbb{E}\left[\left(\boldsymbol{h}_{\mathrm{ul}, l}^{\mathrm{H}} \boldsymbol{\Theta} \boldsymbol{h}_{\mathrm{ul}, l}\right)^{2}\right]
$$

where $\boldsymbol{\Theta}=\boldsymbol{\Psi}^{\mathrm{H}} \boldsymbol{\Gamma} \boldsymbol{\Psi}$ is an eigenvalue decomposition of $\boldsymbol{A}_{l}^{\mathrm{H}} \boldsymbol{A}_{l}$. As a result,

$$
\begin{aligned}
\mathbb{E}\left[\left(\boldsymbol{v}_{l}^{\mathrm{H}} \boldsymbol{v}_{l}\right)^{2}\right] & =\left(\varsigma_{l}^{\prime}\right)^{4} \mathbb{E}\left[\left(\boldsymbol{h}_{\mathrm{ul}, l}^{\mathrm{H}} \boldsymbol{\Gamma} \boldsymbol{h}_{\mathrm{ul}, l}\right)^{2}\right] \\
& =\left(\varsigma_{l}^{\prime}\right)^{4} \mathbb{E}\left[\left(\sum_{p=1}^{P}[\boldsymbol{\Gamma}]_{p, p}\left|\left[\boldsymbol{h}_{\mathrm{ul}, l}\right]_{p}\right|^{2}\right)^{2}\right],
\end{aligned}
$$

where $\left[\boldsymbol{h}_{\mathrm{ul}, l}\right]_{p}$ denotes the $p$-th element of $\boldsymbol{h}_{\mathrm{ul}, l}$. Performing the expectation with respect to $\boldsymbol{h}_{\mathrm{ul}, l}$ and further simplifying yields

$$
\mathbb{E}\left[\boldsymbol{v}_{l}^{\mathrm{H}} \boldsymbol{v}_{l} \boldsymbol{v}_{l}^{\mathrm{H}} \boldsymbol{v}_{l}\right]=\left(\varsigma_{l}^{\prime}\right)^{4}\left\{(\operatorname{tr}[\boldsymbol{\Theta}])^{2}+\operatorname{tr}\left[\boldsymbol{\Theta}^{2}\right]\right\}
$$

This allows (8.19) to be written as

$$
\mathbb{E}\left[\boldsymbol{v}_{l}^{\mathrm{H}} \boldsymbol{v}_{l} \boldsymbol{v}_{l}^{\mathrm{H}} \boldsymbol{v}_{l}\right]=\left(\varsigma_{l}^{\prime}\right)^{4}\left\{\left(\operatorname{tr}\left[\boldsymbol{A}_{l}^{\mathrm{H}} \boldsymbol{A}_{l}\right]\right)^{2}+\operatorname{tr}\left[\boldsymbol{A}_{l}^{\mathrm{H}} \boldsymbol{A}_{l} \boldsymbol{A}_{l}^{\mathrm{H}} \boldsymbol{A}_{l}\right]\right\}
$$

From (8.15), recognizing that the $\operatorname{tr}\left[\boldsymbol{A}_{l}^{\mathrm{H}} \boldsymbol{A}_{l}\right]=P M$ allows one to write

$$
\mathbb{E}\left[\boldsymbol{v}_{l}^{\mathrm{H}} \boldsymbol{v}_{l} \boldsymbol{v}_{l}^{\mathrm{H}} \boldsymbol{v}_{l}\right]=\left(\varsigma_{l}^{\prime}\right)^{4}\left\{P^{2} M^{2}+\operatorname{tr}\left[\left(\boldsymbol{A}_{l}^{\mathrm{H}} \boldsymbol{A}_{l}\right)^{2}\right]\right\}
$$

Substituting back the definition of $\boldsymbol{q}_{l}$, recognizing that $\mathbb{E}\left[\overline{\boldsymbol{h}}_{\mathrm{ul}, l}^{\mathrm{H}} \overline{\boldsymbol{h}}_{\mathrm{ul}, l}\right]=M$, combining (8.21) with the remaining terms in (8.16) and extracting the relevant 
constants yields the desired result in (8.12).

Lemma 8.3.2. Under the same conditions as Lemma 8.3.1,

$$
\begin{aligned}
\iota_{l, k}=\mathbb{E}\left[\left|\boldsymbol{g}_{\mathrm{ul}, l}^{\mathrm{H}} \boldsymbol{g}_{\mathrm{ul}, k}\right|^{2}\right]= & \left(\varsigma_{l}^{\prime}\right)^{2}\left(\varsigma_{k}^{\prime}\right)^{2} \operatorname{tr}\left[\boldsymbol{A}_{k} \boldsymbol{A}_{k}^{\mathrm{H}} \boldsymbol{A}_{l} \boldsymbol{A}_{l}^{\mathrm{H}}\right]+\left(\varsigma_{l}^{\prime}\right)^{2}\left(\bar{\varsigma}_{k}\right)^{2} \operatorname{tr}\left[\overline{\boldsymbol{h}}_{\mathrm{ul}, k}^{\mathrm{H}} \boldsymbol{A}_{l} \boldsymbol{A}_{l}^{\mathrm{H}} \overline{\boldsymbol{h}}_{\mathrm{ul}, k}\right] \\
& +\left(\bar{\varsigma}_{l}\right)^{2}\left(\varsigma_{k}^{\prime}\right)^{2} \operatorname{tr}\left[\overline{\boldsymbol{h}}_{\mathrm{ul}, l}^{\mathrm{H}} \boldsymbol{A}_{k} \boldsymbol{A}_{k}^{\mathrm{H}} \overline{\boldsymbol{h}}_{\mathrm{ul}, l}\right]+\left(\bar{\varsigma}_{l}\right)^{2}\left(\bar{\varsigma}_{k}\right)^{2} \overline{\boldsymbol{h}}_{\mathrm{ul}, l}^{\mathrm{H}} \overline{\boldsymbol{h}}_{\mathrm{ul}, k} \overline{\boldsymbol{h}}_{\mathrm{ul}, k}^{\mathrm{H}} \overline{\boldsymbol{h}}_{\mathrm{ul}, l} .
\end{aligned}
$$

Proof of Lemma 8.3.2. From (8.3), applying the definition of $\boldsymbol{g}_{\mathrm{ul}, l}$ and $\boldsymbol{g}_{\mathrm{ul}, k}$ into $\mathbb{E}\left[\left|\boldsymbol{g}_{\mathrm{ul}, l}^{\mathrm{H}} \boldsymbol{g}_{\mathrm{ul}, k}\right|^{2}\right]$ and denoting $\boldsymbol{v}_{l}=\varsigma_{l}^{\prime} \boldsymbol{A}_{l} \boldsymbol{h}_{\mathrm{ul}, l}$ and $\boldsymbol{q}_{l}=\bar{\varsigma}_{l} \overline{\boldsymbol{h}}_{\mathrm{ul}, l}$ yields

$$
\iota_{l, k}=\mathbb{E}\left[\left|\boldsymbol{g}_{\mathrm{ul}, l}^{\mathrm{H}} \boldsymbol{g}_{\mathrm{ul}, k}\right|^{2}\right]=\mathbb{E}\left[\left|\left(\boldsymbol{v}_{l}^{\mathrm{H}}+\boldsymbol{q}_{l}^{\mathrm{H}}\right)\left(\boldsymbol{v}_{k}+\boldsymbol{q}_{k}\right)\right|^{2}\right]
$$

Expanding and simplifying (8.23) allows one to write

$\iota_{l, k}=\mathbb{E}\left[\left|\boldsymbol{g}_{\mathrm{ul}, l}^{\mathrm{H}} \boldsymbol{g}_{\mathrm{ul}, k}\right|^{2}\right]=\mathbb{E}\left[\left(\boldsymbol{v}_{l}^{\mathrm{H}} \boldsymbol{v}_{k}+\boldsymbol{v}_{l}^{\mathrm{H}} \boldsymbol{q}_{k}+\boldsymbol{q}_{l}^{\mathrm{H}} \boldsymbol{v}_{k}+\boldsymbol{q}_{l}^{\mathrm{H}} \boldsymbol{q}_{k}\right)\left(\boldsymbol{v}_{k}^{\mathrm{H}} \boldsymbol{v}_{l}+\boldsymbol{q}_{k}^{\mathrm{H}} \boldsymbol{v}_{l}+\boldsymbol{v}_{k}^{\mathrm{H}} \boldsymbol{q}_{l}+\boldsymbol{q}_{k}^{\mathrm{H}} \boldsymbol{q}_{l}\right)\right]$

Further expanding and simplifying yields

$\iota_{l, k}=\mathbb{E}\left[\left|\boldsymbol{g}_{\mathrm{ul}, l}^{\mathrm{H}} \boldsymbol{g}_{\mathrm{ul}, k}\right|^{2}\right]=\mathbb{E}\left[\boldsymbol{v}_{l}^{\mathrm{H}} \boldsymbol{v}_{k} \boldsymbol{v}_{k}^{\mathrm{H}} \boldsymbol{v}_{l}\right]+\mathbb{E}\left[\boldsymbol{v}_{l}^{\mathrm{H}} \boldsymbol{q}_{k} \boldsymbol{q}_{k}^{\mathrm{H}} \boldsymbol{v}_{l}\right]+\mathbb{E}\left[\boldsymbol{q}_{l}^{\mathrm{H}} \boldsymbol{v}_{k} \boldsymbol{v}_{k}^{\mathrm{H}} \boldsymbol{q}_{l}\right]+\mathbb{E}\left[\boldsymbol{q}_{l}^{\mathrm{H}} \boldsymbol{q}_{k} \boldsymbol{q}_{k}^{\mathrm{H}} \boldsymbol{q}_{l}\right]$

Upon noting that $\boldsymbol{v}_{l}$ and $\boldsymbol{v}_{k}$ are independent of each other, $\mathbb{E}\left[\boldsymbol{v}_{l} \boldsymbol{v}_{l}^{\mathrm{H}}\right]=\left(\varsigma_{l}^{\prime}\right)^{2} \operatorname{tr}\left[\boldsymbol{A}_{l}^{\mathrm{H}} \boldsymbol{A}_{l}\right]$.

Substituting the definitions of $\boldsymbol{v}_{l}$ and $\boldsymbol{q}_{l}$ into (8.25) and extracting the relevant constants yields

$$
\begin{array}{r}
\iota_{l, k}=\mathbb{E}\left[\left|\boldsymbol{g}_{\mathrm{ul}, l}^{\mathrm{H}} \boldsymbol{g}_{\mathrm{ul}, k}\right|^{2}\right]=\left(\varsigma_{l}^{\prime}\right)^{2}\left(\varsigma_{k}^{\prime}\right)^{2} \mathbb{E}\left[\boldsymbol{h}_{\mathrm{ul}, l}^{\mathrm{H}} \boldsymbol{A}_{l}^{\mathrm{H}} \boldsymbol{A}_{k} \boldsymbol{A}_{k}^{\mathrm{H}}\right]+\left(\varsigma_{l}^{\prime}\right)^{2}\left(\bar{\varsigma}_{k}\right)^{2}\left[\overline{\boldsymbol{h}}_{\mathrm{ul}, k}^{\mathrm{H}} \boldsymbol{A}_{l} \boldsymbol{A}_{l}^{\mathrm{H}} \overline{\boldsymbol{h}}_{\mathrm{ul}, k}\right]+ \\
\left(\bar{\varsigma}_{l}\right)^{2}\left(\varsigma_{k}^{\prime}\right)^{2}\left[\overline{\boldsymbol{h}}_{\mathrm{ul}, l}^{\mathrm{H}} \boldsymbol{A}_{k} \boldsymbol{A}_{k}^{\mathrm{H}} \overline{\boldsymbol{h}}_{\mathrm{ul}, l}\right]+\left(\bar{\varsigma}_{l}\right)^{2}\left(\bar{\varsigma}_{k}\right)^{2}\left[\left|\overline{\boldsymbol{h}}_{\mathrm{ul}, l}^{\mathrm{H}} \overline{\boldsymbol{h}}_{\mathrm{ul}, k}\right|^{2}\right] . \quad(8.26)
\end{array}
$$

Taking the trace of (8.26) and further simplifying yields the desired expression in $(8.22)$.

Lemma 8.3.3. Under the same conditions as Lemma 8.3.1, 


$$
\bar{\chi}_{l}=\mathbb{E}\left[\left\|\boldsymbol{g}_{\mathrm{ul}, l}\right\|^{2}\right]=M\left[P\left(\varsigma_{l}^{\prime}\right)^{2}+\left(\bar{\varsigma}_{l}\right)^{2}\right]
$$

Proof of Lemma 8.3.3. Substituting the definition of $\boldsymbol{g}_{\mathrm{ul}, l}$ into $\chi_{l}$ and expanding allows one to write

$$
\bar{\chi}_{l}=\mathbb{E}\left[\left\|\boldsymbol{g}_{\mathrm{ul}, l}\right\|^{2}\right]=\mathbb{E}\left[\left(\varsigma_{l}^{\prime}\right)^{2} \boldsymbol{h}_{\mathrm{ul}, l}^{\mathrm{H}} \boldsymbol{A}_{l}^{\mathrm{H}} \boldsymbol{A}_{l} \boldsymbol{h}_{\mathrm{ul}, l}\right]+\mathbb{E}\left[\left(\bar{\varsigma}_{l}\right)^{2} \overline{\boldsymbol{h}}_{\mathrm{ul}, l}^{\mathrm{H}} \overline{\boldsymbol{h}}_{\mathrm{ul}, l}\right]
$$

Performing the expectations with respect to $\boldsymbol{h}_{l}$ and extracting the relevant constants yields

$$
\bar{\chi}_{l}=\mathbb{E}\left[\left\|\boldsymbol{g}_{\mathrm{ul}, l}\right\|^{2}\right]=\left(\varsigma_{l}^{\prime}\right)^{2} \operatorname{tr}\left[\boldsymbol{A}_{l}^{\mathrm{H}} \boldsymbol{A}_{l}\right]+\left(\bar{\varsigma}_{l}\right)^{2} \mathbb{E}\left[\overline{\boldsymbol{h}}_{\mathrm{ul}, l}^{\mathrm{H}} \overline{\boldsymbol{h}}_{\mathrm{ul}, l}\right]
$$

Recognizing that $\operatorname{tr}\left[\boldsymbol{A}_{l}^{\mathrm{H}} \boldsymbol{A}_{l}\right]=P M$ and $\mathbb{E}\left[\overline{\boldsymbol{h}}_{\mathrm{ul}, l}^{\mathrm{H}} \overline{\boldsymbol{h}}_{\mathrm{ul}, l}\right]=M$ allows one to write

$$
\bar{\chi}_{l}=\mathbb{E}\left[\left\|\boldsymbol{g}_{\mathrm{ul}, l}\right\|^{2}\right]=M\left[P\left(\varsigma_{l}^{\prime}\right)^{2}+\left(\bar{\varsigma}_{l}\right)^{2}\right]
$$

concluding the proof.

Proposition 8.3.1. With MRC at the BS consisting of a space-constrained ULA, the expected uplink SINR of terminal $l$ in a spatially correlated Ricean fading channel can be approximated as

$$
\mathbb{E}\left[\mathrm{SINR}_{\mathrm{ul}, l}\right] \approx \frac{\rho_{\mathrm{ul}} \beta_{l} \delta_{l}}{\bar{\chi}_{l}+\rho_{\mathrm{ul}} \sum_{\substack{k=1 \\ k \neq l}}^{L} \beta_{k} \iota_{l, k}} .
$$

Method for Proposition 8.3.1. Substituting the results from Lemmas 
8.3.1, 8.3.2 and 8.3.3 for $\delta_{l}, \iota_{l, k}$ and $\bar{\chi}_{l}$ yields the desired expression in (8.31).

Remark 8.3.2. The result in (8.31) is extremely general and is a closed-form solution to a complex scenario, where in addition to fixed physical spacing and MRC at the BS, each terminal has a unique LoS direction, unique Rice factor, unique receive correlation matrix and a unique large scale link gain. It can be readily observed via inspection, that both the numerator and the denominator of (8.31) are influenced by each of the above factors. The result allows for a general evaluation of large MU-MIMO systems with space-constrained ULAs and lends itself to many useful special cases (as shown in Section 8.4). However, before doing this, some important insights from Proposition 8.3.1 are brought to light.

The result in Proposition 8.3.1 is expanded and further simplified on the following page. Due to space restriction, the expressions are presented in landscape style. 


$$
\begin{gathered}
\mathbb{E}\left[\mathrm{SINR}_{\mathrm{ul}, l}\right] \approx \\
\frac{\rho_{\mathrm{ul}} \beta_{l}\left\{\left(\varsigma_{l}^{\prime}\right)^{4}\left\{P^{2} M^{2}+\operatorname{tr}\left[\left(\boldsymbol{A}_{l}^{\mathrm{H}} \boldsymbol{A}_{l}\right)^{2}\right]\right\}+2 P M^{2}\left(\varsigma_{l}^{\prime}\right)^{2}\left(\bar{\varsigma}_{l}\right)^{2}+2\left(\bar{\varsigma}_{l}\right)^{2}\left(\varsigma_{l}^{\prime}\right)^{2} \overline{\boldsymbol{h}}_{\mathrm{ul}, l}^{\mathrm{H}} \boldsymbol{A}_{l} \boldsymbol{A}_{l}^{\mathrm{H}} \overline{\boldsymbol{h}}_{\mathrm{ul}, l}+\left(\bar{\varsigma}_{l}\right)^{4} M^{2}\right\}}{M P+\rho_{\mathrm{ul}} \sum_{\substack{k=1 \\
k \neq l}}^{L} \beta_{k}\left\{\left(\varsigma_{l}^{\prime}\right)^{2}\left(\varsigma_{k}^{\prime}\right)^{2} \operatorname{tr}\left[\boldsymbol{A}_{k} \boldsymbol{A}_{k}^{\mathrm{H}} \boldsymbol{A}_{l} \boldsymbol{A}_{l}^{\mathrm{H}}\right]+\left(\varsigma_{l}^{\prime}\right)^{2}\left(\bar{\varsigma}_{k}\right)^{2} \operatorname{tr}\left[\overline{\boldsymbol{h}}_{\mathrm{ul}, k}^{\mathrm{H}} \boldsymbol{A}_{l} \boldsymbol{A}_{l}^{\mathrm{H}} \overline{\boldsymbol{h}}_{\mathrm{ul}, k}\right]+\left(\bar{\varsigma}_{l}\right)^{2}\left(\varsigma_{k}^{\prime}\right)^{2}\left[\overline{\boldsymbol{h}}_{\mathrm{ul}, l}^{\mathrm{H}} \boldsymbol{A}_{k} \boldsymbol{A}_{k}^{\mathrm{H}} \overline{\boldsymbol{h}}_{\mathrm{ul}, l}\right]+\left(\bar{\varsigma}_{l}\right)^{2}\left(\bar{s}_{k}\right)^{2}\left[\left|\overline{\boldsymbol{h}}_{\mathrm{ul}, l}^{\mathrm{H}} \overline{\boldsymbol{h}}_{\mathrm{ul}, k}\right|^{2}\right]\right\}},
\end{gathered}
$$

when substituting the respective terms in the numerator and the denominator. Substituting the definition of $\varsigma_{l}^{\prime}$ and $\bar{\varsigma}_{l},(8.32)$ can $\stackrel{N}{V}$ be simplified further to give

$$
\begin{aligned}
& \mathbb{E}\left[\operatorname{SINR}_{\mathrm{ul}, l]}\right] \approx \frac{\rho_{\mathrm{ul}} \beta_{l}\left\{\frac{1}{P^{2}\left(1+K_{l}\right)^{2}}\left\{P^{2} M^{2}+\operatorname{tr}\left[\left(\boldsymbol{A}_{l}^{\mathrm{H}} \boldsymbol{A}_{l}\right)^{2}\right]\right\}+\frac{2 M^{2} K_{l}}{\left(K_{l}+1\right)^{2}}+\frac{2 K_{l}}{P\left(K_{l}+1\right)^{2}} \overline{\boldsymbol{h}}_{\mathrm{ul}, l}^{\mathrm{H}} \boldsymbol{A}_{l} \boldsymbol{A}_{l}^{\mathrm{H}} \overline{\boldsymbol{h}}_{\mathrm{ul}, l}+\frac{K_{l}^{2}}{\left(K_{l}+1\right)^{2}} M^{2}\right\}}{M P+\rho_{\mathrm{ul}} \sum_{\substack{k=1 \\
k \neq l}}^{L} \beta_{k}\left\{\frac{\operatorname{tr}\left[\boldsymbol{A}_{k} \boldsymbol{A}_{k}^{\mathrm{H}} \boldsymbol{A}_{l} \boldsymbol{A}_{l}^{\mathrm{H}}\right]}{P^{2}\left(K_{k}+1\right)\left(K_{l}+1\right)}+\frac{\left(\overline{\boldsymbol{h}}_{\mathrm{u}, k}^{\mathrm{H}}, \boldsymbol{A}_{l} \boldsymbol{A}_{l}^{\mathrm{H}} \overline{\boldsymbol{h}}_{\mathrm{ul}, k}\right) K_{k}}{P\left(K_{l}+1\right)\left(K_{k}+1\right)}+\frac{\left(\overline{\bar{u}}_{\mathrm{u}, l}^{\mathrm{H}} \boldsymbol{A}_{k} \boldsymbol{A}_{k}^{\mathrm{H}} \overline{\boldsymbol{h}}_{\mathrm{ul}, l}\right) K_{l}}{P\left(K_{l}+1\right)\left(K_{k}+1\right)}+\frac{K_{k} K_{l}}{\left(K_{l}+1\right)\left(K_{k}+1\right)}\left|\overline{\boldsymbol{h}}_{\mathrm{ul}, l}^{\mathrm{H}} \overline{\boldsymbol{h}}_{\mathrm{ul}, k}\right|^{2}\right\}} \\
& =\frac{\frac{\rho_{\mathrm{u} \mid \beta_{l}}}{P^{2}\left(1+K_{l}\right)^{2}}\left\{P^{2} M^{2}\left(1+2 K_{l}+K_{l}^{2}\right)+\operatorname{tr}\left[\left(\boldsymbol{A}_{l}^{\mathrm{H}} \boldsymbol{A}_{l}\right)^{2}\right]+\frac{2}{P} K_{l} \overline{\boldsymbol{h}}_{\mathrm{ul}, l}^{\mathrm{H}} \boldsymbol{A}_{l} \boldsymbol{A}_{l}^{\mathrm{H}} \overline{\boldsymbol{h}}_{\mathrm{ul}, l}\right\}}{M P+\rho_{\mathrm{ul}} \sum_{\substack{k=1 \\
k \neq l}}^{L} \frac{\beta_{k}}{P^{2}\left(K_{k}+1\right)\left(K_{l}+1\right)}\left\{\operatorname{tr}\left[\boldsymbol{A}_{k} \boldsymbol{A}_{k}^{\mathrm{H}} \boldsymbol{A}_{l} \boldsymbol{A}_{l}^{\mathrm{H}}\right]+K_{k} P\left(\overline{\boldsymbol{h}}_{\mathrm{ul}, k}^{\mathrm{H}} \boldsymbol{A}_{l} \boldsymbol{A}_{l}^{\mathrm{H}} \overline{\boldsymbol{h}}_{\mathrm{ul}, k}\right)+K_{l} P\left(\overline{\boldsymbol{h}}_{\mathrm{ul}, l}^{\mathrm{H}} \boldsymbol{A}_{k} \boldsymbol{A}_{k}^{\mathrm{H}} \overline{\boldsymbol{h}}_{\mathrm{ul}, l}\right)+K_{k} K_{l}\left|\overline{\boldsymbol{h}}_{\mathrm{ul}, l}^{\mathrm{H}} \overline{\boldsymbol{h}}_{\mathrm{ul}, k}\right|^{2}\right\}} .
\end{aligned}
$$


Remark 8.3.3. A number of important insights can be drawn from (8.33). It can be observed that for any particular system dimensions and link SNR, both the numerator and the denominator of (8.33) contain quadratic forms of the type $\overline{\boldsymbol{h}}_{\mathrm{ul}}^{\mathrm{H}} \boldsymbol{A} \boldsymbol{A}^{\mathrm{H}} \overline{\boldsymbol{h}}_{\mathrm{ul}}$. Via the Rayleigh quotient theorem (see e.g., [18]), such quadratic forms are maximized when $\overline{\boldsymbol{h}}_{\mathrm{ul}}$ is parallel (aligned) to the maximum eigenvector of $\boldsymbol{A}_{l} \boldsymbol{A}_{l}^{\mathrm{H}}$. From this, an interesting observation can be made: Alignment of $\overline{\boldsymbol{h}}_{\mathrm{ul}, l}$ with $\boldsymbol{A}_{l} \boldsymbol{A}_{l}^{\mathrm{H}}$ amplifies the expected signal power for terminal $l$, maximizing the quadratic form in the numerator of (8.33). In contrast to this, alignment of $\overline{\boldsymbol{h}}_{\mathrm{ul}, k}$ with $\boldsymbol{A}_{l} \boldsymbol{A}_{l}^{\mathrm{H}}, \overline{\boldsymbol{h}}_{\mathrm{ul}, l}$ with $\boldsymbol{A}_{k} \boldsymbol{A}_{k}^{\mathrm{H}}$ and $\overline{\boldsymbol{h}}_{\mathrm{ul}, l}^{\mathrm{H}}$ with $\overline{\boldsymbol{h}}_{\mathrm{ul}, k}$ amplifies the expected interference power for terminal $l$, leading to a lower expected SINR. Likewise, if $\boldsymbol{A}_{k} \boldsymbol{A}_{k}^{\mathrm{H}}$ and $\boldsymbol{A}_{l} \boldsymbol{A}_{l}^{\mathrm{H}}$ are aligned, i.e., alignment of the long-term preferential directions of propagation channel from terminals $l$ and $k$, then $\operatorname{tr}\left[\boldsymbol{A}_{k} \boldsymbol{A}_{k}^{\mathrm{H}} \boldsymbol{A}_{l} \boldsymbol{A}_{l}^{\mathrm{H}}\right]$ increases, further degrading the expected SINR. It is worth mentioning that this observation was made in Chapter 7, when analyzing the expected SINR performance of MF precoding with unequal spatial correlation matrices under Rayleigh fading. Therefore, the global phenomena is that the expected SINR reduces by virtue of channel similarities of various types (LoS and spatial correlation), and increases if the channels are more diverse.

It is worth noting that (8.31) (and in turn (8.33)) can be further used to approximate the ergodic sum spectral efficiency of the system by

$$
\mathbb{E}\left[\mathrm{R}_{\mathrm{sum}, \mathrm{ul}}\right] \approx \sum_{l=1}^{L} \log _{2}\left(1+\mathbb{E}\left[\mathrm{SINR}_{\mathrm{ul}, l}\right]\right)
$$

The accuracy of the derived closed-form approximations in (8.31) (and in turn in (8.33)) and (8.34) is demonstrated in Section 8.5. In the following section, three special cases of (8.33) are presented to demonstrate its generality.

\subsection{Special Cases}

Corollary 8.4.1. With MRC at the BS consisting of a SC ULA, the expected uplink SINR of terminal $l$ with no LoS, i.e, Rayleigh fading with unequal corre- 
lation matrices for each terminal, can be approximated as

$$
\mathbb{E}\left[\mathrm{SINR}_{\mathrm{ul}, l}\right] \approx \frac{\rho_{\mathrm{ul}} \frac{\beta_{l}}{P^{2}}\left\{P^{2} M^{2}+\operatorname{tr}\left[\left(\boldsymbol{A}_{l}^{\mathrm{H}} \boldsymbol{A}_{l}\right)^{2}\right]\right\}}{M P+\rho_{\mathrm{ul}} \sum_{\substack{k=1 \\ k \neq l}}^{L} \frac{\beta_{k}}{P^{2}}\left\{\operatorname{tr}\left[\boldsymbol{A}_{k} \boldsymbol{A}_{k}^{\mathrm{H}} \boldsymbol{A}_{l} \boldsymbol{A}_{l}^{\mathrm{H}}\right]\right\}} .
$$

Proof of Corollary 8.4.1. Substituting $K_{l}=K_{k}=0, \forall l, k=1, \ldots, L$ in (8.33) yields the desired expression in (8.35).

Corollary 8.4.2 (Proposition 1 in [93]). With MRC processing at the BS containing of a SC ULA, the expected uplink SINR for terminal $l$ with no LoS and equal correlation matrices, i.e., Rayleigh fading with a fixed correlation for each terminal, can be approximated as

$$
\mathbb{E}\left[\mathrm{SINR}_{\mathrm{ul}, l}\right] \approx \frac{\rho_{\mathrm{ul}} \frac{\beta_{l}}{P^{2}}\left\{P^{2} M^{2}+\operatorname{tr}\left[\left(\boldsymbol{A}_{l}^{\mathrm{H}} \boldsymbol{A}_{l}\right)^{2}\right]\right\}}{M P+\rho_{\mathrm{ul}} \sum_{\substack{k=1 \\ k \neq l}}^{L} \frac{\beta_{k}}{P^{2}}\left\{\operatorname{tr}\left[\left(\boldsymbol{A}_{l} \boldsymbol{A}_{l}^{\mathrm{H}}\right)^{2}\right]\right\}} .
$$

Proof of Corollary 8.4.2. Setting $\boldsymbol{A}_{l}=\boldsymbol{A}_{k}, \forall l, k=1, \ldots, L$ in (8.35) gives the desired result. Note that the result is consistent with [93].

Corollary 8.4.3. With MRC at the BS consisting of a SC ULA, the expected uplink SINR of terminal $l$ with LoS i.e., correlated Ricean fading, with equal correlation matrices for each terminal can be approximated as

$\mathbb{E}\left[\mathrm{SINR}_{\mathrm{ul}, l}\right] \approx$

$$
\frac{\frac{\rho_{\mathrm{ul}} \beta_{l}}{P^{2}\left(1+K_{l}\right)^{2}}\left\{P^{2} M^{2}\left(1+2 K_{l}+K_{l}^{2}\right)+\operatorname{tr}\left[\left(\boldsymbol{A}_{l}^{\mathrm{H}} \boldsymbol{A}_{l}\right)^{2}\right]+\frac{2}{P} K_{l} \overline{\boldsymbol{h}}_{\mathrm{ul}, l}^{\mathrm{H}} \boldsymbol{A}_{l} \boldsymbol{A}_{l}^{\mathrm{H}} \overline{\boldsymbol{h}}_{\mathrm{ul}, l}\right\}}{M P+\rho_{\mathrm{ul}} \sum_{\substack{k=1 \\ k \neq l}}^{L} \frac{\beta_{k}}{P^{2}\left(K_{k}+1\right)\left(K_{l}+1\right)}\left\{\operatorname{tr}\left[\left(\boldsymbol{A}_{l} \boldsymbol{A}_{l}^{\mathrm{H}}\right)^{2}\right]+K_{k} P\left(\overline{\boldsymbol{h}}_{\mathrm{ul}, k}^{\mathrm{H}} \boldsymbol{A}_{l} \boldsymbol{A}_{l}^{\mathrm{H}} \overline{\boldsymbol{h}}_{\mathrm{ul}, k}\right)+K_{l} P\left(\overline{\boldsymbol{h}}_{\mathrm{ul}, l}^{\mathrm{H}} \boldsymbol{A}_{l} \boldsymbol{A}_{l}^{\mathrm{H}} \overline{\boldsymbol{h}}_{\mathrm{ul}, l}\right)+K_{k} K_{l}\left|\overline{\boldsymbol{h}}_{\mathrm{ul}, l}^{\mathrm{H}} \overline{\boldsymbol{h}}_{\mathrm{ul}, k}\right|^{2}\right\}} .
$$

Proof of Corollary 8.4.3. Replacing $\boldsymbol{A}_{k}=\boldsymbol{A}_{l}$ in (8.33) yields the desired 
expression in (8.37), concluding the proof.

Remark 8.4.1. Corollaries 8.4.1 and 8.4.2 share a common trend in that both the numerators and denominators are governed by spatial correlation matrices in $\boldsymbol{A}_{l}$ and $\boldsymbol{A}_{k}$, respectively. In the case where correlation matrices are fixed for each terminal, the trace in their respective denominators can be readily seen to translate from $\operatorname{tr}\left[\boldsymbol{A}_{k} \boldsymbol{A}_{k}^{\mathrm{H}} \boldsymbol{A}_{l} \boldsymbol{A}_{l}^{\mathrm{H}}\right]$ to $\operatorname{tr}\left[\left(\boldsymbol{A}_{l}^{\mathrm{H}} \boldsymbol{A}_{l}\right)^{2}\right]$. Likewise, for Corollary 8.4.3, as the case of equal correlation matrices is considered for all terminals, $\boldsymbol{A}_{k}=\boldsymbol{A}_{l}$, $\forall l, k=1, \ldots, L$ in $(8.33)$ resulting in (8.37).

In the subsequent section, the convergence of the expected SINR and ergodic sum spectral efficiency is analyzed with MRC, as the number of BS antennas, $M$, grows without bound with a fixed number of user terminals, $L$.

\subsection{Limiting Expected SINR and Ergodic Sum Spectral Efficiency Analysis}

Proposition 8.3.1 presents an expected uplink SINR approximation for terminal $l$ which is suitable for any system size, as well as any link SNR, LoS level, spatial correlation level and physical array spacing. The asymptotic behavior of (8.31) (and in turn of (8.33)) and (8.34), as $M \rightarrow \infty$, with a fixed (finite) $L$ is now examined. Firstly, dividing the numerator and denominator of (8.31) (and in turn of (8.33)) by $M^{2}$, the limit can be observed as

$$
\mathbb{E}\left[\mathrm{SINR}_{\mathrm{ul}, l}^{\lim }\right]=\lim _{M \rightarrow \infty}\left\{\frac{\rho_{\mathrm{ul}} \frac{\beta_{l}}{M^{2}} \delta_{l}}{\frac{\bar{\chi}_{l}}{M^{2}}+\rho_{\mathrm{ul}} \sum_{\substack{k=1 \\ k \neq l}}^{L} \beta_{k}\left(\frac{\iota_{l, k}}{M^{2}}\right)}\right\}
$$

The numerator of (8.38) can now be written as

$$
\frac{\rho_{\mathrm{ul} \beta_{l}}}{M^{2}} \delta_{l}=\frac{\rho_{\mathrm{ul}} \beta_{l}}{M^{2}}\left(\delta_{l}^{1}+\delta_{l}^{2}+\delta_{l}^{3}\right),
$$


where $\delta_{l}^{1}, \delta_{l}^{2}$ and $\delta_{l}^{3}$ are the three quantities in the numerator of (8.31) (and in turn in (8.33)). Likewise, the denominator of (8.38) can be written as

$$
\frac{\bar{\chi}_{l}}{M^{2}}+\rho_{\mathrm{ul}} \sum_{\substack{k=1 \\ k \neq l}}^{L} \beta_{k}\left(\frac{\iota_{l, k}}{M^{2}}\right)=\frac{\bar{\chi}_{l}}{M^{2}}+\rho_{\mathrm{ul}} \sum_{\substack{l=1 \\ l \neq k}}^{L} \beta_{k}\left(\iota_{l, k}^{1}+\iota_{l, k}^{2}+\iota_{l, k}^{3}+\iota_{l, k}^{4}\right)
$$

where $\iota_{l, k}^{1}, \iota_{l, k}^{2}, \iota_{l, k}^{3}$ and $\iota_{l, k}^{4}$ are the four quantities in the denominator of (8.31) (and therefore in (8.33)).

Referring to the numerator of (8.38), it is interesting to observe that two terms in

$$
\delta_{l}^{1}=\left(\varsigma_{l}^{\prime}\right)^{4}\left\{\frac{\operatorname{tr}\left[\left(\boldsymbol{A}_{l}^{\mathrm{H}} \boldsymbol{A}_{l}\right)^{2}\right]}{M^{2}}\right\}
$$

and

$$
\delta_{l}^{2}=2\left(\varsigma_{l}^{\prime}\right)^{2}\left(\bar{\varsigma}_{l}\right)^{2}\left\{\frac{\overline{\boldsymbol{h}}_{\mathrm{ul}, l}^{\mathrm{H}} \boldsymbol{A}_{l} \boldsymbol{A}_{l}^{\mathrm{H}} \overline{\boldsymbol{h}}_{\mathrm{ul}, l}}{M^{2}}\right\},
$$

do not vanish from $\delta_{l}$ as $M$ grows without bound, while the denominator of (8.38) has four terms in $\iota_{l, k}$, which do not vanish as $M \rightarrow \infty$. These are,

$$
\begin{gathered}
\iota_{l, k}^{1}=\left(\varsigma_{l}^{\prime}\right)^{2}\left(\varsigma_{k}^{\prime}\right)^{2}\left\{\frac{\operatorname{tr}\left[\boldsymbol{A}_{k} \boldsymbol{A}_{k}^{\mathrm{H}} \boldsymbol{A}_{l} \boldsymbol{A}_{l}^{\mathrm{H}}\right]}{M^{2}}\right\}, \\
\iota_{l, k}^{2}=\left(\varsigma_{l}^{\prime}\right)^{2}\left(\bar{\varsigma}_{k}\right)^{2}\left\{\frac{\operatorname{tr}\left[\overline{\boldsymbol{h}}_{\mathrm{ul}, k}^{\mathrm{H}} \boldsymbol{A}_{l} \boldsymbol{A}_{l}^{\mathrm{H}} \overline{\boldsymbol{h}}_{\mathrm{ul}, k}\right]}{M^{2}}\right\}, \\
\iota_{l, k}^{3}=\left(\bar{\varsigma}_{l}\right)^{2}\left(\varsigma_{k}^{\prime}\right)^{2}\left\{\frac{\operatorname{tr}\left[\overline{\boldsymbol{h}}_{\mathrm{ul}, l}^{\mathrm{H}} \boldsymbol{A}_{k} \boldsymbol{A}_{k}^{\mathrm{H}} \overline{\boldsymbol{h}}_{\mathrm{ul}, l}\right]}{M^{2}}\right\},
\end{gathered}
$$

and

$$
\iota_{l, k}^{4}=\left(\bar{\varsigma}_{l}\right)^{2}\left(\bar{\varsigma}_{k}\right)^{2}\left\{\frac{\overline{\boldsymbol{h}}_{\mathrm{ul}, l}^{\mathrm{H}} \overline{\boldsymbol{h}}_{\mathrm{ul}, k} \overline{\boldsymbol{h}}_{\mathrm{ul}, k}^{\mathrm{H}} \overline{\boldsymbol{h}}_{\mathrm{ul}, l}}{M^{2}}\right\} .
$$

In the sequel, Lemmas 8.5.1, 8.5.2 and 8.5.3 derive the deterministic limits of (8.41) - (8.46), respectively. 
Lemma 8.5.1. $\lim _{M \rightarrow \infty} \iota_{l, k}^{4}$ is given by

$$
\begin{aligned}
& \bar{\iota}_{l, k}^{4}=\left(\bar{\varsigma}_{l}\right)^{2}\left(\bar{\varsigma}_{k}\right)^{2}\left\{\lim _{M \rightarrow \infty}\left\{\left|\frac{\overline{\boldsymbol{h}}_{\mathrm{ul}, l}^{\mathrm{H}} \overline{\boldsymbol{h}}_{\mathrm{ul}, k}}{M}\right|^{2}\right\}\right\} \\
&=\left(\bar{\varsigma}_{l}\right)^{2}\left(\bar{\varsigma}_{k}\right)^{2} \vartheta\left(\bar{\phi}_{l}, \bar{\phi}_{k}\right)^{2}
\end{aligned}
$$

where $\vartheta\left(\bar{\phi}_{l}, \bar{\phi}_{k}\right)=\left|\operatorname{sinc}\left(\pi \frac{d_{0}}{\lambda}\left(\sin \left(\bar{\phi}_{l}\right)-\sin \left(\bar{\phi}_{k}\right)\right)\right)\right|$. Here sinc $(\cdot)$ denotes the sinc function and $d_{0}$ is the total length of the ULA at the BS.

Proof of Lemma 8.5.1. One can take note of the fact that

$$
\begin{aligned}
\vartheta\left(\bar{\phi}_{l}, \bar{\phi}_{k}\right) & =\lim _{M \rightarrow \infty}\left\{\left|\frac{\overline{\boldsymbol{h}}_{\mathrm{ul}, l}^{\mathrm{H}} \overline{\boldsymbol{h}}_{\mathrm{ul}, k}}{M}\right|\right\} \\
& =\lim _{M \rightarrow \infty}\left\{\left|\frac{1}{M} \sum_{c=0}^{M-1} e^{j 2 \pi \frac{c}{\lambda} \frac{d_{0}}{M-1}\left(\sin \left(\bar{\phi}_{l}\right)-\sin \left(\bar{\phi}_{k}\right)\right)}\right|\right\} \\
& =\left|\int_{0}^{1} e^{j 2 \pi \frac{d_{0}}{\lambda}\left(\sin \left(\bar{\phi}_{l}\right)-\sin \left(\bar{\phi}_{k}\right)\right) f} d f\right| \\
& =\left|\operatorname{sinc}\left(\pi \frac{d_{0}}{\lambda}\left(\sin \left(\bar{\phi}_{l}\right)-\sin \left(\bar{\phi}_{k}\right)\right)\right)\right|
\end{aligned}
$$

using the limit of a Riemann sum to represent an integral. Taking the square of $\vartheta\left(\bar{\phi}_{l}, \bar{\phi}_{k}\right)$ yields the desired result in (8.47).

Remark 8.5.1. The expression in (8.48) is another closed-form solution and can be readily seen to be dependent on the respective LoS angles unique to terminals $l$ and $k$.

Lemma 8.5.2. $\lim _{M \rightarrow \infty} \iota_{l, k}^{3}$ is given by

$$
\bar{\iota}_{l, k}^{3}=\left(\bar{\varsigma}_{l}\right)^{2}\left(\varsigma_{k}^{\prime}\right)^{2}\left\{\lim _{M \rightarrow \infty}\left\{\frac{\operatorname{tr}\left[\overline{\boldsymbol{h}}_{\mathrm{ul}, l}^{\mathrm{H}} \boldsymbol{A}_{k} \boldsymbol{A}_{k}^{\mathrm{H}} \overline{\boldsymbol{h}}_{\mathrm{ul}, l}\right]}{M^{2}}\right\}\right\}=\left(\bar{\varsigma}_{l}\right)^{2}\left(\varsigma_{k}^{\prime}\right)^{2} \sum_{p=1}^{P} \vartheta\left(\bar{\phi}_{l}, \phi_{k, p}\right)^{2}
$$

Proof of Lemma 8.5.2. Expanding the trace in (8.49), one can state 


$$
\begin{aligned}
& \bar{\iota}_{l, k}^{3}=\left(\bar{\varsigma}_{l}\right)^{2}\left(\varsigma_{k}^{\prime}\right)^{2}\left\{\lim _{M \rightarrow \infty}\left\{\frac{\operatorname{tr}\left[\overline{\boldsymbol{h}}_{\mathrm{ul}, l}^{\mathrm{H}} \boldsymbol{A}_{k} \boldsymbol{A}_{k}^{\mathrm{H}} \overline{\boldsymbol{h}}_{\mathrm{ul}, l}\right]}{M^{2}}\right\}\right\} \\
& =\left(\bar{\varsigma}_{l}\right)^{2}\left(\varsigma_{k}^{\prime}\right)^{2}\left\{\lim _{M \rightarrow \infty}\left\{\frac{1}{M^{2}} \overline{\boldsymbol{h}}_{\mathrm{ul}, l}^{\mathrm{H}}\left\{\left[\begin{array}{llll}
\boldsymbol{a}\left(\phi_{k, 1}\right) & \boldsymbol{a}\left(\phi_{k, 2}\right) & \ldots & \boldsymbol{a}\left(\phi_{k, P}\right)
\end{array}\right]\left[\begin{array}{c}
\boldsymbol{a}^{\mathrm{H}}\left(\phi_{k, 1}\right) \\
\boldsymbol{a}^{\mathrm{H}}\left(\phi_{k, 2}\right) \\
\vdots \\
\boldsymbol{a}^{\mathrm{H}}\left(\phi_{k, P}\right)
\end{array}\right]\right\} \overline{\boldsymbol{h}}_{\mathrm{ul}, l}\right\}\right\} \\
& =\left(\bar{\varsigma}_{l}\right)^{2}\left(\varsigma_{k}^{\prime}\right)^{2}\left\{\lim _{M \rightarrow \infty}\left\{\frac{1}{M^{2}} \sum_{p=1}^{P}\left|\boldsymbol{a}\left(\bar{\phi}_{l}\right)^{\mathrm{H}} \boldsymbol{a}\left(\phi_{k, p}\right)\right|^{2}\right\}\right\} \\
& =\left(\bar{\varsigma}_{l}\right)^{2}\left(\varsigma_{k}^{\prime}\right)^{2} \sum_{p=1}^{P} \vartheta\left(\bar{\phi}_{l}, \phi_{k, p}\right)^{2} \text {. }
\end{aligned}
$$

This yields the desired result, concluding the proof.

Remark 8.5.2. Note that as $\iota_{l, k}^{2}$ and $\delta_{l}^{2}$ have a similar structure to $\iota_{l, k}^{3}$, the limiting values of $\iota_{l, k}^{2}$ and $\delta_{l}^{2}$ in $\bar{\iota}_{l, k}^{2}$ and $\bar{\delta}_{l}^{2}$ have the same form as (8.49), with the exception that the angles in $\vartheta(\cdot)$ are replaced with $\bar{\phi}_{k}, \phi_{l, r}$ for $\bar{\iota}_{l, k}^{2}$ and $\bar{\phi}_{l}, \phi_{l, r}$ for $\bar{\delta}_{l}^{2}$, respectively. Furthermore, both $\bar{\iota}_{l, k}^{2}$ and $\bar{\delta}_{l}^{2}$ will need to have the necessary scaling of $\left(\varsigma_{l}^{\prime} \bar{\varsigma}_{k}\right)^{2}$ and $2\left(\varsigma_{l}^{\prime}\right)^{2}\left(\bar{\varsigma}_{l}\right)^{2}$, as shown in (8.44) and (8.42), respectively.

Lemma 8.5.3. $\lim _{M \rightarrow \infty} \iota_{l, k}^{1}$ is given by

$$
\begin{aligned}
\bar{\iota}_{l, k}^{1} & =\left(\varsigma_{l}^{\prime}\right)^{2}\left(\varsigma_{k}^{\prime}\right)^{2}\left\{\lim _{M \rightarrow \infty}\left\{\frac{\operatorname{tr}\left[\boldsymbol{A}_{k} \boldsymbol{A}_{k}^{\mathrm{H}} \boldsymbol{A}_{l} \boldsymbol{A}_{l}^{\mathrm{H}}\right]}{M^{2}}\right\}\right\} \\
& =\left(\varsigma_{l}^{\prime}\right)^{2}\left(\varsigma_{k}^{\prime}\right)^{2} \sum_{r=1}^{P} \sum_{t=1}^{P} \vartheta\left(\phi_{k, r}, \phi_{l, t}\right)^{2} .
\end{aligned}
$$

Proof of Lemma 8.5.3. One can notice that

$$
\begin{aligned}
\bar{\iota}_{l, k}^{1} & =\left(\varsigma_{l}^{\prime}\right)^{2}\left(\varsigma_{k}^{\prime}\right)^{2}\left\{\lim _{M \rightarrow \infty}\left\{\frac{\operatorname{tr}\left[\boldsymbol{A}_{k} \boldsymbol{A}_{k}^{\mathrm{H}} \boldsymbol{A}_{l} \boldsymbol{A}_{l}^{\mathrm{H}}\right]}{M^{2}}\right\}\right\} \\
& =\left(\varsigma_{l}^{\prime}\right)^{2}\left(\varsigma_{k}^{\prime}\right)^{2}\left\{\lim _{M \rightarrow \infty}\left\{\frac{\operatorname{tr}\left[\boldsymbol{A}_{k}^{\mathrm{H}} \boldsymbol{A}_{l} \boldsymbol{A}_{l}^{\mathrm{H}} \boldsymbol{A}_{k}\right]}{M^{2}}\right\}\right\} .
\end{aligned}
$$


Then,

$$
\begin{aligned}
\bar{\iota}_{l, k}^{1} & =\left(\varsigma_{l}^{\prime}\right)^{2}\left(\varsigma_{k}^{\prime}\right)^{2}\left\{\lim _{M \rightarrow \infty}\left\{\frac{\operatorname{tr}\left[\boldsymbol{A}_{k}^{\mathrm{H}} \boldsymbol{A}_{l} \boldsymbol{A}_{l}^{\mathrm{H}} \boldsymbol{A}_{k}\right]}{M^{2}}\right\}\right\} \\
& =\left(\varsigma_{l}^{\prime}\right)^{2}\left(\varsigma_{k}^{\prime}\right)^{2}\left\{\lim _{M \rightarrow \infty}\left\{\frac{1}{M^{2}} \sum_{r=1}^{P} \boldsymbol{a}\left(\phi_{k, r}\right)^{\mathrm{H}} \boldsymbol{A}_{l} \boldsymbol{A}_{l}^{\mathrm{H}} \boldsymbol{a}\left(\phi_{k, r}\right)\right\}\right\} \\
& =\left(\varsigma_{l}^{\prime}\right)^{2}\left(\varsigma_{k}^{\prime}\right)^{2}\left\{\lim _{M \rightarrow \infty}\left\{\frac{1}{M^{2}} \sum_{r=1}^{P} \sum_{t=1}^{P}\left|\boldsymbol{a}\left(\phi_{k, r}\right)^{\mathrm{H}} \boldsymbol{a}\left(\phi_{l, t}\right)\right|^{2}\right\}\right\} \\
& =\left(\varsigma_{l}^{\prime}\right)^{2}\left(\varsigma_{k}^{\prime}\right)^{2} \frac{1}{M^{2}} \sum_{r=1}^{P} \sum_{t=1}^{P} \vartheta\left(\phi_{k, r} \phi_{l, t}\right)^{2},
\end{aligned}
$$

giving the desired result.

Remark 8.5.3. Note that $\delta_{l}^{1}$ has a similar form to $\iota_{l, k}^{1}$. Using the same methodology as in Lemma 8.5.3, one can obtain $\bar{\delta}_{l}^{1}$, the limiting value of $\delta_{1}^{1}$, where the angles in $\vartheta(\cdot)$ will be replaced by $\phi_{l, r}, \phi_{l, t}$ with $\left(\varsigma_{l}^{\prime}\right)^{4}$ providing the required scaling.

Theorem 8.5.1. The limiting uplink SINR for terminal $l$ with MRC and a SC ULA at the BS can be written as

$$
\mathbb{E}\left[\mathrm{SINR}_{\mathrm{ul}, l}^{\lim }\right]=\frac{\rho_{\mathrm{ul}} \beta_{l}\left(\bar{\delta}_{l}^{1}+\bar{\delta}_{l}^{2}\right)}{\rho_{\mathrm{ul}} \sum_{\substack{k=1 \\ k \neq l}}^{L} \beta_{k}\left(\bar{\iota}_{l, k}^{1}+\bar{\iota}_{l, k}^{2}+\bar{\iota}_{l, k}^{3}+\bar{\iota}_{l, k}^{4}\right)} .
$$

Proof of Theorem 8.5.1. Using the results from Lemmas 8.5.1, 8.5.2, 8.5.3 and keeping in mind Remarks 8.5.2 and 8.5.3 yields the desired expression.

As such the limiting ergodic sum spectral efficiency is given by

$$
\mathbb{E}\left[\mathrm{R}_{\text {sum }, \mathrm{ul}}^{\lim }\right]=\sum_{l=1}^{L} \log _{2}\left(1+\mathbb{E}\left[\operatorname{SINR}_{\mathrm{ul}, l}^{\lim }\right]\right)
$$

Remark 8.5.4. It is noteworthy that in the limit, the results presented in (8.54) and (8.55) are exact, as the variance of the denominator in (8.54) approaches zero with $M \rightarrow \infty$. A rigorous mathematical proof of this can be found in Appendix I of [68].

In the following section, the tightness of the closed-form approximations 


\begin{tabular}{ccc}
\hline \hline Parameter & \multicolumn{2}{c}{ Value } \\
\cline { 2 - 3 } & Microwave & mmWave \\
\hline Carrier frequency $[\mathrm{GHz}]$ & 2 & 28 \\
LoS attenuation exponent $[\alpha]$ & 2.2 & 2 \\
NLoS attenuation exponent & 3.67 & 2.92 \\
LoS shadow fading standard deviation $\left[\sigma_{\mathrm{sh}}\right]$ & 3 & 5.8 \\
NLoS shadow fading standard deviation & 4 & 8.7 \\
K-Factor mean [dB] & 9 & $12[129]$ \\
$K$-Factor standard deviation $[\mathrm{dB}]$ & 5 & $3[129]$ \\
\hline
\end{tabular}

Table 8.1: Parameters used for the generation of numerical results.

derived in Sections 8.4 and 8.5 is evaluated.

\subsection{Numerical Results and Discussion}

In this section, numerical results are presented to evaluate the analytical approximations in Sections 8.4 and 8.5 for a SC ULA with fixed physical space, $\bar{d}_{0}$. The ULA receives simultaneous uplink data streams from $L$ single-antenna user terminals in the same time-frequency resource. Unless otherwise specified, the parameters used for the numerical results are specified in Table 8.1 for an UMi scenario. For all numerical results, propagation parameters for the microwave and mmWave frequency bands were obtained from [136] and [137], respectively. A circular cell having a radius $100 \mathrm{~m}$ is considered with an exclusion radius of $d_{0}=10 \mathrm{~m}$. A uniform distribution of terminals is assumed in the cell coverage area and $10^{4}$ Monte-Carlo realizations for each result are considered. The parameter $v$ is chosen such that the instantaneous per-terminal SINR is $0 \mathrm{~dB}, 95 \%$ of the time, at link $\mathrm{SNR}\left(\rho_{\mathrm{ul}}\right)=0 \mathrm{~dB}$, for the system dimensions of $M=256$ and $L=32$.

In line with the numerical results presented in Chapter 5 of the thesis, given a link distance $r_{l}$, a statistical approach is employed in determining whether the terminal experiences LoS or NLoS conditions on the uplink to the BS. For the 
microwave case, the probability of terminal $l$ experiencing LoS is governed by

$$
P_{\mathrm{LoS}}\left(r_{l}\right)=\left(\min \left(18 / r_{l}, 1\right)\left(1-e^{-r_{l} / 36}\right)\right)+e^{-r_{l} / 36}
$$

Naturally, the probability of the terminal experiencing NLoS is then determined by

$$
P_{\mathrm{NLOS}}=1-P_{\mathrm{LoS}}
$$

Equivalently, for the mmWave case [137],

$$
P_{\mathrm{LoS}}\left(r_{l}\right)=\left(1-P_{\text {out }}\left(r_{l}\right)\right) e^{-\omega_{\mathrm{LoS}} r_{l}},
$$

where $1 / \omega_{\mathrm{LoS}}=67.1$ meters and $P_{\text {out }}$ is the outage probability, occurring when the combined geometric attenuation and shadow fading in either the LoS or NLoS states is sufficiently large. In this chapter, for simplicity, $P_{\text {out }}=0$ when determining the LoS and NLoS probabilities. Upon determining the link state of each terminal, the corresponding link parameters are selected to model the largescale propagation effects of geometric attenuation and shadow-fading. These are as specified in Table 8.1. A unique $K$-factor, $K_{l}$, is assigned to the wireless link from the $l$-th terminal to the BS from a log-normal distribution with the mean and standard deviation specified in Table 8.1. This is referred to in the subsequent numerical results as $K_{l} \sim \ln$ (mean, standard deviation).

The accuracy of the proposed expected per-terminal SINR in (8.29) is examined. Fig. 8.1 illustrates the expected SINR of a given terminal as a function of $\rho_{\mathrm{ul}}$ (link SNR) for a system with $M=256$ and $L=32, P=50$ and $\bar{d}_{0}=8 \lambda$. In addition to the microwave and mmWave cases, the correlated Rayleigh fading case is considered for comparison purposes. Also, the case where each terminal is assigned a fixed $K$-factor of $5 \mathrm{~dB}$ is considered. Three trends can be observed: Firstly, transitioning from large to small angular spread $\left(\Delta \sim \mathcal{U}\left[\frac{-\pi}{2}, \frac{\pi}{2}\right]\right.$ to $\left.\Delta \sim \mathcal{U}\left[\frac{-\pi}{16}, \frac{\pi}{16}\right]\right)$ tends to significantly reduce the expected SINR for all cases. This is despite the fact that the ULA contains very large numbers of antenna elements at the BS, and is due to the reduction in the spatial diversity (rank) 


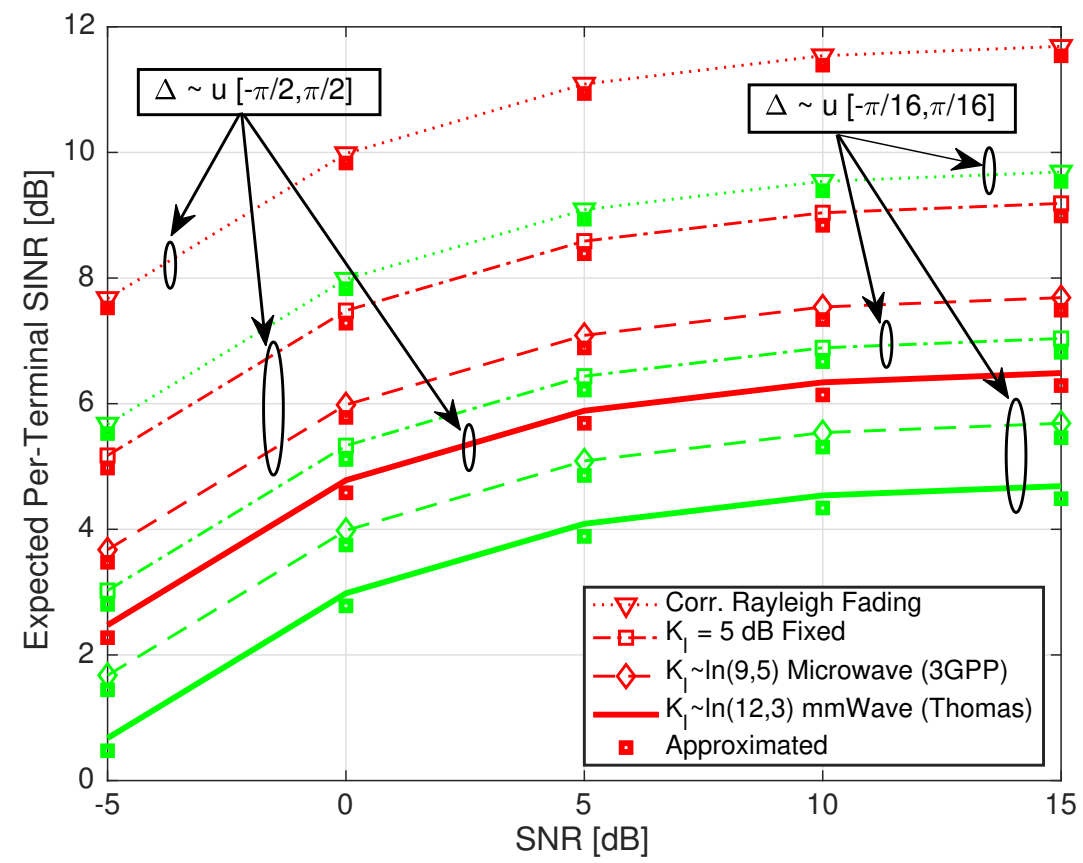

Figure 8.1: Expected per-terminal SINR vs. $\rho_{\mathrm{ul}}$ (link SNR) with $M=256, L=$ $32, P=50, \bar{d}_{0}=8 \lambda$.

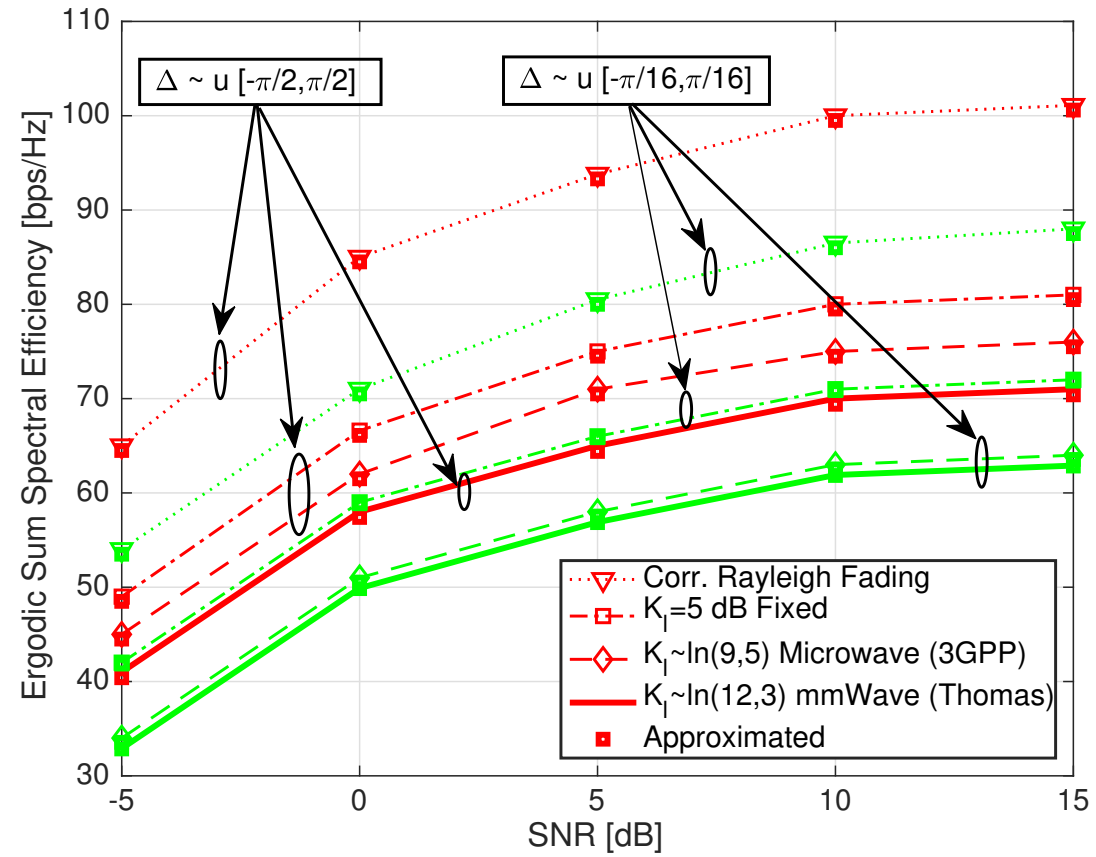

Figure 8.2: Ergodic sum spectral efficiency vs. $\rho_{\mathrm{ul}}$ (link SNR) with $M=$ $256, L=32, P=50, \bar{d}_{0}=8 \lambda$. 
of the uplink channel, allowing the BS array to only see a very narrow spread of incoming power. Secondly, increasing the mean of the $K$-factor has an adverse effect on the expected SINR. This is because a stronger specular component in the channel tends to reduce the multi-path diversity and in turn reduces the overall spatial selectivity of the channel. Equivalently, this can be interpreted as an increase in the overall level of correlation in the channel leading to an increase in the inter-terminal interference and lower per-terminal SINR. Third, our proposed approximations are seen to remain extremely tight for the entire link SNR range for all cases. The analytical expressions are also seen to remain tight for the special case presented in Corollary 8.4.1 (8.35), where each terminal undergoes Rayleigh fading with unequal correlation matrices. In addition to the above, the expected per-terminal SINR in each case is seen to saturate with growing link SNR, due to the inability of the MRC to mitigate the remaining inter-terminal interference. The expected per-terminal SINR is translated to an ergodic sum spectral efficiency via (8.34). This is depicted in Fig. 8.2, where similar conclusions to Fig. 8.1 can be drawn.

Considering the special cases in Corollary 8.4.2 (8.36) and Corollary 8.4.3 (8.37), the influence of LoS, as well as equal and unequal correlation matrices on the ergodic sum spectral efficiency is examined. This is demonstrated in Fig. 8.3. Using the same system parameters from Fig. 8.1 and Fig. 8.2, (listed in the figure captions) at $\rho_{\mathrm{ul}}(\operatorname{link} \mathrm{SNR})=10 \mathrm{~dB}$, the cumulative distribution functions (CDFs) of the derived ergodic sum spectral efficiency approximation in (8.34) is compared with its simulated counterparts. Note that the CDF is obtained by averaging over the fast-fading in the channel with each value representing the variations in the link gains and $K$-factors. Notice that irrespective of the underlaying propagation characteristics (Rayleigh or Ricean fading), unequal correlation matrices results in a higher ergodic sum spectral efficiency of the system allowing the ULA to leverage a larger amount of spatial diversity. Furthermore, it is again observed that a stronger specular component tends to decrease the ergodic sum spectral efficiency. The derived approximations are robust to the presence of equal and unequal correlation matrices, as well as 


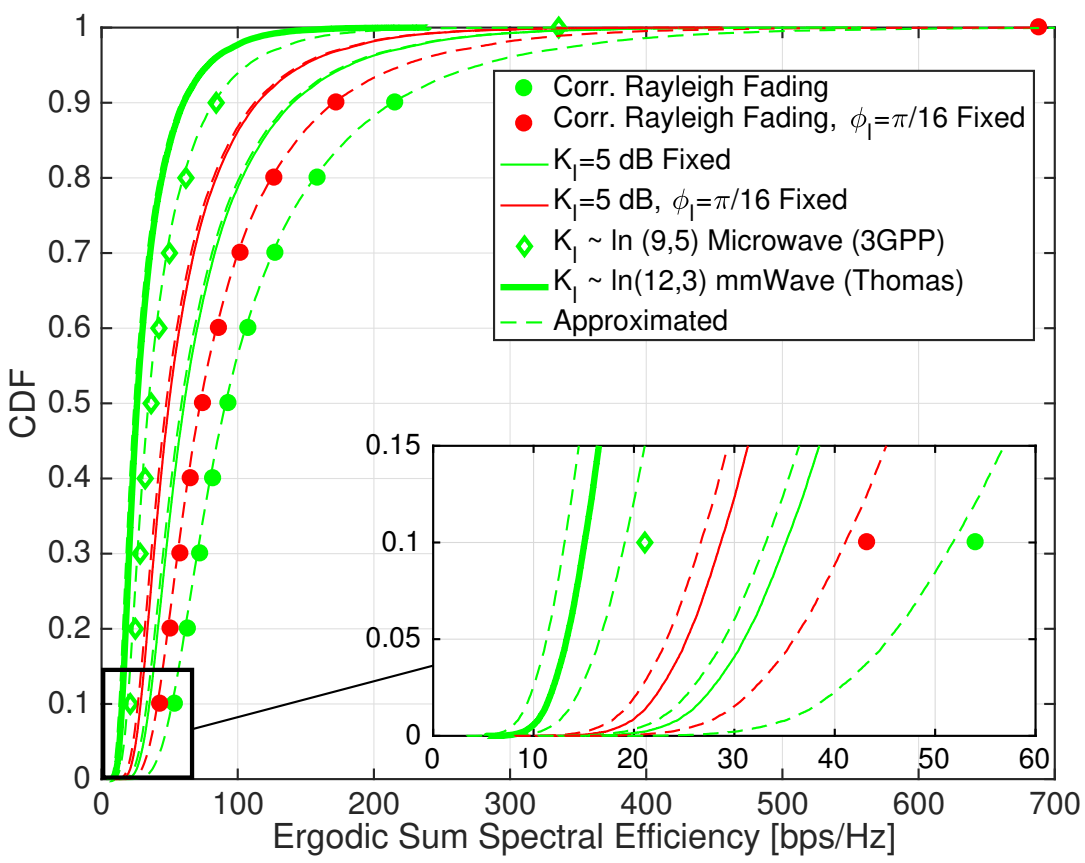

Figure 8.3: Ergodic sum spectral efficiency $\mathrm{CDF}$ with $M=256, L=32$, $\rho_{\mathrm{ul}}\left(\right.$ link SNR) $=10 \mathrm{~dB}, P=50, \bar{d}_{0}=8 \lambda$ and $\Delta \sim u\left[-\frac{\pi}{16}, \frac{\pi}{16}\right]$ (unless specified in the figure).

changes in the level of LoS.

Next, the accuracy of the limiting expected per-terminal SINR expression derived in (8.54) is inspected with growing numbers of BS antennas and a fixed number of terminals, where $L=32$. This is depicted in Fig. 8.4. Three trends can be observed from the result: After recognizing that increasing $M$ (the number of receive antennas at the ULA) increases the expected per-terminal SINR, for each case the expected SINR slowly saturates with growing $M$ and approaches its limiting value at approximately 500 antenna elements for each case, respectively. This is a result of channels from multiple terminals becoming asymptotically orthogonal. ${ }^{5}$ Secondly, decreasing the physical size of the array further reduces the inter-element spacing, reducing the spatial diversity which then translates into a reduction in the expected SINR for all cases respectively. Furthermore, one can observe that each case converges to the derived limiting value. The limiting expected per-terminal SINR was then translated into a

\footnotetext{
${ }^{5}$ In the large MU-MIMO literature, this effect has been famously coined as favorable propagation $[9,57]$.
} 


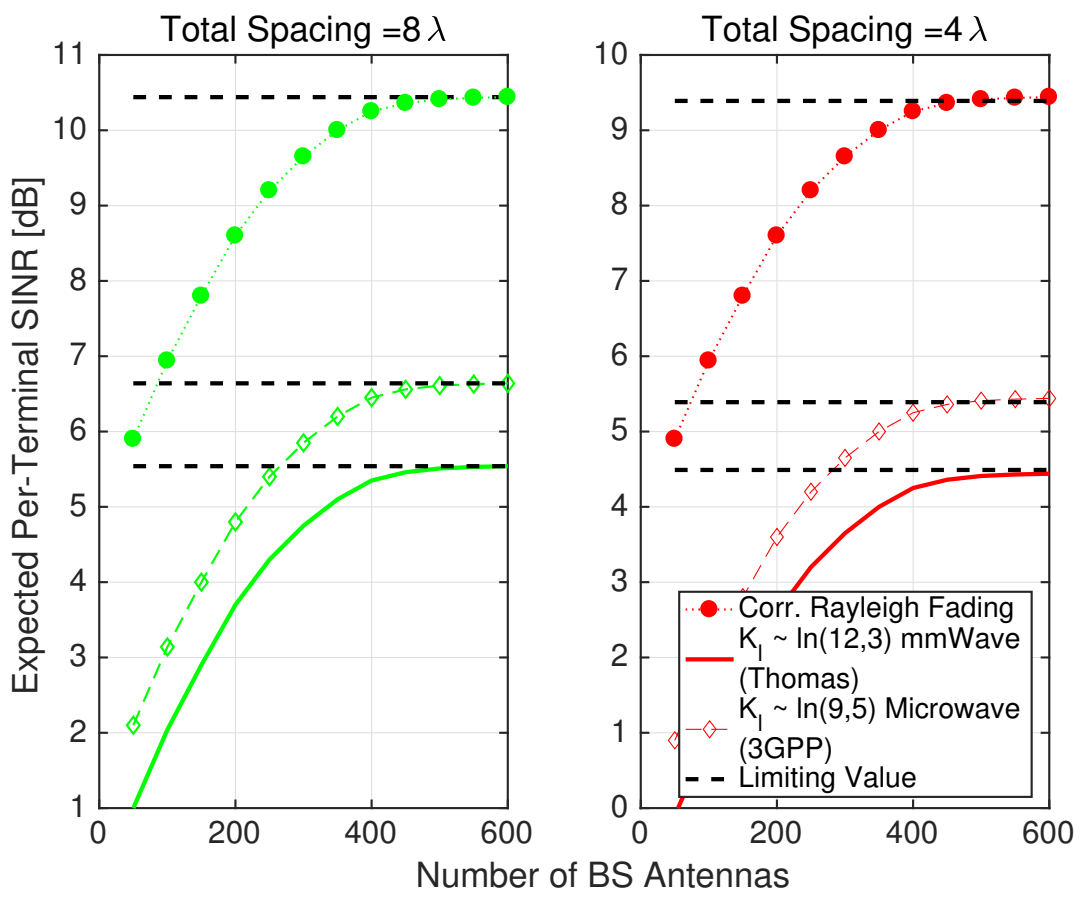

Figure 8.4: Expected per-terminal SINR vs. $M$ with $L=32$ at $\rho_{\mathrm{ul}}$ (link SNR) $=10 \mathrm{~dB}, P=50, \Delta \sim u\left[-\frac{\pi}{16}, \frac{\pi}{16}\right]$.

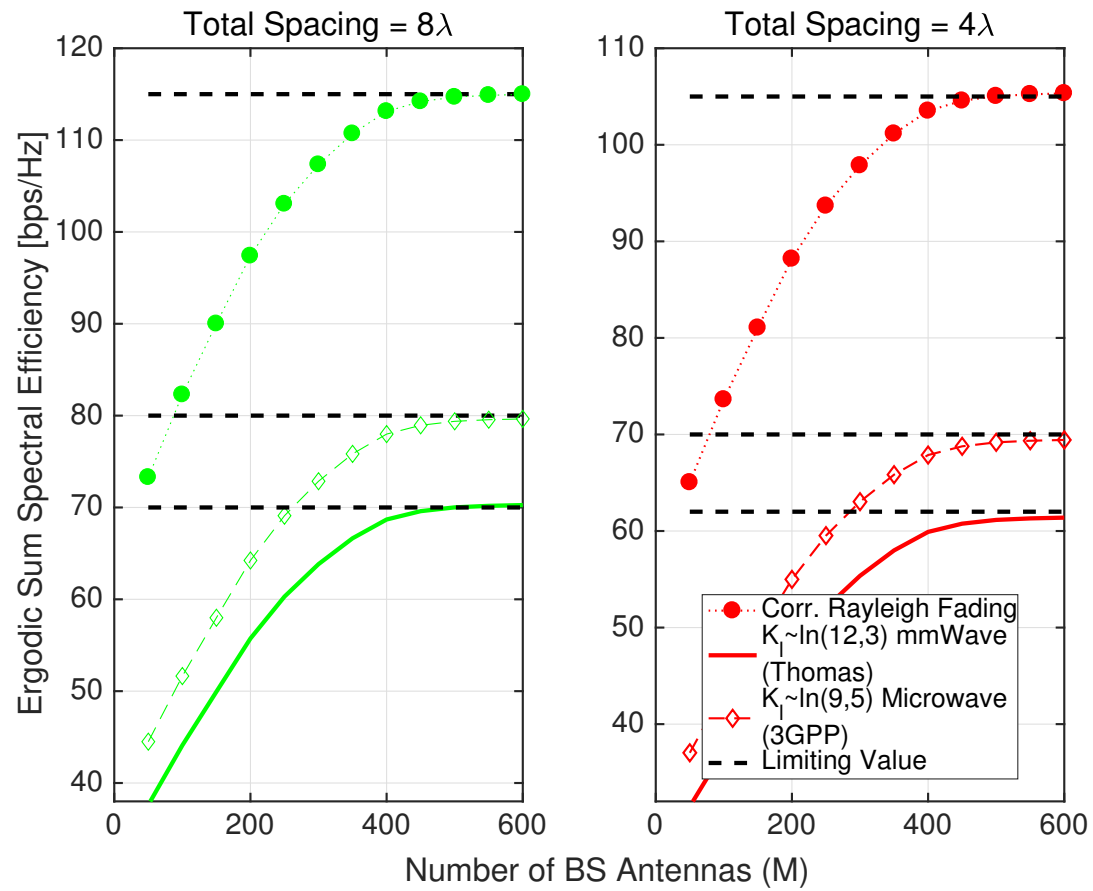

Figure 8.5: Ergodic sum spectral efficiency vs. $M$ with $L=32$ at $\rho_{\mathrm{ul}}$ (link SNR) $=10 \mathrm{~dB}, P=50, \Delta \sim u\left[-\frac{\pi}{16}, \frac{\pi}{16}\right]$. 
limiting ergodic sum spectral efficiency via (8.55), as shown in Fig. 8.5. Here similar trends to Fig. 8.4 can be found for both $\bar{d}_{0}=8 \lambda$ and $\bar{d}_{0}=4 \lambda$.

\subsection{Concluding Remarks}

In this chapter, the uplink performance of large MU-MIMO systems under spatially correlated Ricean fading is investigated with ULAs at the BS employed in a fixed physical space. Closed-form approximations to the expected per-terminal SINR and ergodic sum spectral efficiency are derived with MRC processing at the BS. In the limit of a large number of BS antennas, asymptotic expressions for the expected per-terminal SINR and ergodic sum spectral efficiency were derived. Our numerical results show that with constraints on the physical size of the ULA, the expected SINR saturates with increasing link SNR and BS antenna numbers. The analysis accommodates changes in system dimensions, link SNR, LoS levels, spatial correlation levels and variation in fixed physical spacings. Unequal correlation matrices at each terminal result in a performance increase, whilst LoS had an adverse impact on system performance. 


\section{Chapter 9}

\section{Conclusions and Future Work}

\subsection{Thesis Summary}

Analyzing the spectral efficiency performance of conventional and large-scale multiuser multiple-input multiple-output (MU-MIMO) systems in heterogeneous channel conditions holds the key to a more thorough understanding of the performance of current and future cellular systems. The increasing pressure to operate future wireless systems in commensurately smaller areas with large antenna arrays at both microwave and millimeter-wave (mmWave) frequency bands, is likely to raise the amount of heterogeneity present in propagation channels. Accordingly, this thesis analyzes and evaluates the performance of conventional and large MU-MIMO systems with linear transceiver architectures to theoretically characterize their performance in scenarios of current and future interest. In doing so, the thesis presents a variety of analytical techniques to tackle the complex statistics resulting from heterogeneous propagation channels. A number of practically useful insights on the performance of conventional and large MU-MIMO systems were obtained, leading to a deeper understanding of such systems in diverse propagation environments. 


\subsection{Chapter Summary and Concluding Remarks}

Following the Introduction chapter, the thesis presented a general overview of statistical channel models and random matrix theory results which are specific to MIMO communication systems. Along with known results, novel joint probability density functions for the complex correlated central and uncorrelated noncentral Wishart distributed channel correlation matrices were presented. An overview of conventional and large MU-MIMO systems was then presented with a particular emphasis on uplink and downlink multiuser digital signal processing techniques. A number of shortcomings in these technologies were identified, which further motivated the research presented in the contributions chapters of the thesis. A summary and concluding remarks for each contributions chapter of the thesis is presented below:

- Chapter 4 proposes a general analytical framework for characterizing the expected per-terminal signal-to-interference-plus-noise-ratio (SINR) and ergodic sum spectral efficiency of a coordinated two-tier small cellular system. Overall, the key contributions of the chapter are:

- With regularized zero-forcing (RZF) precoding, under i.i.d. and semicorrelated Rayleigh fading, with equal correlation matrices, the analytical expressions were averaged over the eigenvalue densities of the respective complex Wishart matrices derived in Chapter 2. In the high signal-to-noise-ratio (SNR) regime, ZF precoding was considered and closed-form expressions of the above performance metrics were derived with unequal spatial correlation matrices for each terminal.

- Base station coordination is central to interference limited smallcellular systems, directly influencing the gains in the expected SINR and ergodic spectral efficiencies. Both of these performance metrics were also influenced by small-cell locations and by variation in the degrees of BS coordination, as they directly impact the system's ability 
to suppress dominant interferers. With semi-correlated fading, the expected SINR decreased with increasing levels of spatial correlation due to a loss in the usable spatial degrees of freedom. Expected SINR and ergodic spectral efficiencies with unequal correlation matrices were observed to be greater than the case with equal correlation matrices, as predicted mathematically.

- The impact of dominant line-of-sight (LoS) directions on the terminal SINR and system spectral efficiency is investigated in Chapter 5, assuming a Ricean fading propagation channel and RZF precoding on the downlink. The key contributions of the chapter are as follows:

- Analytical expressions for the expected SINR and ergodic sum spectral efficiency are derived. The derived expressions are averaged with respect to the previously unknown arbitrary eigenvalue densities of the complex uncorrelated non-central Wishart matrix, as presented and derived in Chapter 2. Unlike previous studies, a unique Rice factor is modeled for each terminal, where the considered system model caters for terrain variations across multiple terminals, making it suitable for analysis of current and future systems. In the high SNR regime, a tight distributional approximation to the ZF per-terminal SNR is presented with a gamma distribution, whose parameters are derived.

- With both the microwave and mmWave propagation parameters, the presented numerical results suggest that increasing the specular component of the propagation channel has an adverse effect on the expected SINR and ergodic sum spectral efficiency. On the contrary, increasing the variability of Rice factor enhances the peak ergodic sum spectral efficiency, while reducing the median and cell-edge spectral efficiencies. The evaluation of the variability of Rice factors is novel and can help to identify the sensitivities in multiuser system performance. 
- Furthermore, both the developed approximations are insensitive to changes in the system dimensions, link SNRs and unequal levels of LoS, respectively. Previous approximations were unable to handle such heterogeneous channels.

- A simplified methodology to approximate the uplink and downlink MUMIMO system performance with a moderate number of BS antennas was presented in Chapter 6. A general class of linear transceivers was considered, where the main conclusions of the chapter were:

- Under i.i.d. Rayleigh fading, the instantaneous and expected perterminal SINR and SNR were approximated with maximum-ratio combining, ZF combining and minimum-mean-squared-error (MMSE) combining on the uplink, and matched-filter (MF), ZF and RZF precoding on the downlink. The approximation methodology is based on the identification of specific components in the above mentioned performance metrics which tend to stabilize for moderate numbers of BS antennas, and replacing only these specific components by their expected values.

- In general, the resulting expressions allows for clear insights to be drawn into the factors which most contribute to the above mentioned performance metrics. Numerical evaluation of the approximations demonstrate they are extremely accurate and stable for moderate numbers of BS antennas. The approximation methodology is are robust to the type of fading environment assumed, and easily extended to suit other statistical channel models, such as spatially correlated Ricean fading.

- Chapter 7 mathematically proves that the widely used, yet overly simplified spatial correlation models that result in common spatial correlation matrices for each terminal under estimate the expected SINR and ergodic sum spectral efficiency performance of the system. As a result performance with equal correlation structures acts as a useful lower bound. In 
contrast to this, the use of more complex and physically motivated remote scattering models results in unequal spatial correlation structures which lead to higher performance. The main contributions can be summarized as follows:

- Assuming semi-correlated Rayleigh fading, with MF and ZF precoding, closed-form approximations to the expected SINR, expected SNR and ergodic sum spectral efficiency of a MU-MIMO system are derived. In general, the derived expressions demonstrate that equal correlation structures tend to maximize the expected interference power and hence lead to a lower SINR. The results derived are the simplest form of results derived for such complex systems avoiding the previously proposed iterative solutions, such as fixed point algorithms. The derived approximations remain accurate for all non-physical and physically motivated spatial correlation models.

- Overall, the results from this chapter demonstrate the sensitivity of the achievable performance using different spatial correlation models. Such a sensitivity analysis is useful in providing a cautionary tale of its impact to potential analysts, who may not have access to expensive empirical channel measurements.

- Chapter 8 presents the final contributions chapter of the thesis, where the performance of uplink MRC MU-MIMO system is studied. Assuming a uniform linear array (ULA) at the BS with a fixed total physical spacing, a general framework for approximating the expected SINR and ergodic sum spectral efficiency is developed under spatially correlated Ricean fading channels. Unequal receive spatial correlation levels, unequal LoS levels, and unequal link gains are considered. The derived analysis leads to numerous insights regarding the impact of LoS and spatial correlation on the space-constrained system performance. In particular, the alignment of a desired spatial correlation matrix with the desired LoS direction amplifies the expected signal power, while the alignment of interfering correlation 
matrix with the desired LoS direction tends to increase the expected interference power. The global phenomena emerging from this is that the expected SINR reduces by virtue of channel similarities of various types (LoS and spatially correlated), and increases if the channels are more diverse. Other major conclusions of the chapter are as follows:

- The chapter also extends and generalizes a prior physical spaceconstrained channel model, by including the effects of unequal levels of receive spatial correlation, unequal levels of LoS and unequal link gains for each terminal.

- Deterministic limits of the SINR and sum spectral efficiency are analyzed when the number of service antennas are increased indefinitely with a fixed number of terminals.

- Numerical results demonstrate that a space-constrained ULA deployment with MRC processing causes saturation of the expected SINR and ergodic sum spectral efficiency with increasing link SNR and BS antennas. Several special cases of the derived expressions are also presented when only non LoS components are present with equal and unequal spatial correlation matrices, as well as when each terminal having LoS components has equal correlation matrices.

This concludes the major contributions of the thesis.

\subsection{Future Work}

The conclusions developed throughout the thesis motivate further investigations in unexplored research areas directly related to the contents of the thesis. These are identified and discussed in the following section. More specifically, the author wishes to propose the following specific research areas for further exploration. 


\subsubsection{MU-MIMO Analysis With Spatial Channel Models}

It is known that the propagation characteristics at microwave and mmWave frequency bands are vastly different, with experimental validation showing that mmWave systems experience much larger geometric attenuation, blockages and diffraction $[12,128,130,131,137,175]$. This requires large antenna arrays to close the link budget and to overcome the high electromagnetic losses $[11,13]$. In turn, this may lead the propagation channel to illuminate fewer dominant scatterers [137]. While the research community is still investigating and understanding the true nature of mmWave channels, it is often claimed that mmWave channels are sparser than microwave channels $[12,127,137,153,165,176]$. Due to this increased sparsity, the propagation channel can not be statistically modeled via a complex Gaussian distribution, an assumption made throughout the thesis to capture rich scattering propagation environments.

To accurately model mmWave channels and its physical artifacts, cluster based spatial channel models (also known as Saleh-Valenzuela type channel models) with a finite number of scatterers and paths are often considered [128, 131$133,136,137,164,177]$. Such models are heavily influenced by the random geometry of the propagation environment along with the transmit and receive far-field antenna array responses, making it extremely difficult to make any analytical progress in terms of theoretical predictions of the maximum achievable spectral efficiency with linear transmit and receive processing techniques. ${ }^{1}$ Naturally, one can numerically simulate such systems to gain an understanding of how they operate. However, for a more fundamental understanding of the dominant parameters that influence system performance, analytical progress has to be made with such models.

In order to solve the above mentioned problem, the thesis introduces several possible approaches, such as the simplified analysis methodology in Chapter 6, as well as the Neumann Series expansion in Chapters 4 and 7 to approximate a wide range of SINR and sum spectral efficiency performance metrics. Therefore,

\footnotetext{
${ }^{1}$ This is particularly an issue with higher dimensional antenna arrays, such as the uniform rectangular and uniform cylindrical arrays which utilize both the azimuth and zenith domains.
} 
an attempt to extend the current analysis in the thesis to cluster based spatial channel models should be made using the these analytical methods. If one is able to successfully make progress, such solutions will allow for further design and optimization of transceiver signal processing techniques, as well forming a basis for system evaluation and design of mmWave cellular systems.

\subsubsection{Terminals vs. Pilots for Large MU-MIMO Systems}

As the focus of the thesis was to get an analytical grasp on conventional and large MU-MIMO systems operating in heterogeneous channel conditions, idealistic assumptions such as availability of perfect channel state information (CSI) at the BS and absence of pilot contamination were made. Naturally, these assumptions are hard to justify in practice and therefore such imperfections have to be considered in the analysis of MU-MIMO systems in order to better understand their performance in heterogeneous environments. To this end, performance analysis of large MU-MIMO with such imperfections are of significant interest and importance. ${ }^{2}$ Here, it would be sensible to assume the time-division duplex (TDD) protocol to acquire the instantaneous CSI at the service array due to its scalability (see footnote 3), where the uplink and downlink transmission takes place in the same frequency resource but are separated in time. ${ }^{3}$ This means that the physical propagation channels are reciprocal, i.e., the channel responses are the equivalent in both the uplink and downlink directions with the necessary radio frequency $(\mathrm{RF})$ calibration on the respective paths. ${ }^{4}$

\footnotetext{
${ }^{2}$ Naturally, these results would also be interesting to observe for conventional MU-MIMO systems.

${ }^{3}$ This time separation is considered within one transmission time interval of a classical time-frequency grid with orthogonal frequency division multiplexing waveforms. Moreover, there are several good reasons for operating large MU-MIMO system in TDD mode. First, only the BS needs to know the channel responses to coherently process the received signal $[9$, $11,57,79]$. Second, the uplink estimation overhead is proportional to the number of terminals, but independent of the BS antennas (which is the case for frequency-division duplex systems), making the TDD protocol scalable with respect to the large number of service antennas. Furthermore, from basic estimation theory, it is known that the estimation quality (perantenna) cannot be reduced by adding more antennas at the BS. In fact, the estimation quality improves with the BS antennas if there is a known correlation structure between the channel responses over the array [79, 178].

${ }^{4}$ Calibration in the uplink and downlink is necessary since the transceiver hardware is generally not reciprocal $[11,57,79]$. Fortunately, the uplink and downlink mismatches only
} 
Until now, the majority of the research on large MU-MIMO systems has focused on establishing the fundamental physical layer (PHY) properties. In contrast to this, the communications fraternity has only briefly introduced the resource allocation problems, usually handled in the medium access control (MAC) layer, despite the fact that their true spectral efficiency performance can be better understood if the PHY and MAC layers are jointly optimized. For a multicellular system, the importance of resource allocation for large MUMIMO was described in [179], where initial guidelines were given. A main insight was that the limited number of orthogonal pilot sequences needs to be allocated intelligently among the terminals to reduce interference, which can be done by capitalizing on geometric attenuation differences $[180,181]$ and spatial correlation [178].

In this context, a related resource allocation question of how many terminals should be scheduled per-cell to maximize spectral efficiency is of interest. This question has only been partially answered by the authors in [181], who consider i.i.d. Rayleigh fading channels with unequal link gains for each terminals. To this date, the validity of the conclusions in [181] for more heterogeneous propagation channels, such as unequally correlated Ricean fading still remains an open problem. It is anticipated that factors such as the unequal correlation matrices, unequal levels of LoS, coherence block length, number of BS antennas and total number of terminals will determine the answer to the above question. Keeping this in mind, on the downlink, analytical expressions for the expected SINR and ergodic sum spectral should be derived with MF, ZF and RZF linear processing. Numerical results should focus on answering the key question of the number of terminals which can successfully be scheduled given a fixed number of service antennas, such that the spectral efficiency performance is maximized.

change by a few degrees over a one-hour period and can be mitigated by relatively simple calibration methods without extra reference transceivers, by relying on the coupling between the antennas in the service array [79]. 


\subsubsection{Large MU-MIMO With Double Scattering}

Large antenna arrays complement the operation of future MU-MIMO systems in the millimetric frequency bands $[11-13,127]$. At such frequencies, the high electromagnetic losses rapidly degrade the quality of the received power at a given terminal. For this reason, such systems aim to serve a commensurately smaller area in comparison to contemporaneous cellular architectures. In order to minimize the propagation losses at mmWave frequency bands, research has proposed a reduction in the small-cell BS height (see for e.g., [182] and references therein). As an artifact of this, scattering near the BS is also likely to influence the channel impulse responses to multiple terminals. Naturally, if the terminals are also in cluttered environments, the differences in their geographical location leads to variations in their angular spreads. As explored in Chapter 7 of the thesis, in spatially correlated propagation environments, this leads to differences in the correlation patterns over the terrain of multiple terminals. Furthermore, a difference in the angular spread around the BS array is now also to be expected, since lowering the BS height allows the service array to see far more scatterers than it would otherwise. The work presented in Chapter 7 only considers the impact of the remote scattering models on the terminal side of the link, which captures the differences in the correlation patterns by assuming that the BS is elevated and is free from local obstructions within its vicinity.

To extend this, the performance of large MU-MIMO systems should be explored under double scattering channel models, which utilizes the geometry of the propagation environment to model spatial fading correlation. Thanks to its flexibility, this model has gained a vast amount of interest in multiple antenna communication $[183,184]$. However, these works do not consider large multicellular MU-MIMO systems with linear processing and pilot contamination under heterogeneous fading channels. Analytical progress on such models is also slow due to the complexity involved in modeling the overall propagation channel. As a result, an attempt to derive the expected terminal SINR and ergodic sum spectral efficiency should be made with double scattering models considering 
the above level system imperfections.

This concludes the future work component of the chapter.

\subsection{Final Remarks}

Overall, this thesis has presented a variety of analytical techniques and approaches to analyze the performance of conventional and large MU-MIMO systems with linear transceiver architectures. The author hopes that the analytical results and evaluation presented in the thesis contribute to a more fundamental understanding of MU-MIMO systems and their operation in heterogeneous environments, forming a basis for further research in this area. The work presented in this thesis has led to 8 peer-reviewed publications in highly regarded journals and conferences. 


\section{Bibliography}

[1] FCC, FCC auctions advanced wireless services (AWS-3), FCC, Mar. 2015.

[2] Cisco, Cisco visual networking index: Global mobile data traffic forecast update 2013-2018, Cisco, Mar. 2015.

[3] ITU-R, Technical report on the minimum requirements related to the performance of IMT-2020 radio interface(s), ITU, Oct. 2016.

[4] C. E. Shannon, "A mathematical theory of communication," Bell Systems Tech. Journal, vol. 27, pp. 379-423, Oct. 1948.

[5] G. J. Foschini, K. Karakayali, and R. A. Valenzuela, "Network coordination for spectrally efficient communications in cellular systems," IEEE Wireless Commun., vol. 13, no. 4, pp. 56-61, Aug. 2006.

[6] E. Teletar, "Capacity of multi-antenna Gaussian channels," European Trans. Telecommun., vol. 10, no. 6, pp. 585-595, Dec. 1999.

[7] J. H. Winters, J. Salz, and R. D. Gitlin, "The impact of antenna diversity on the capacity of wireless communication systems," IEEE Trans. Commun., vol. 42, no. 4, pp. 1740-1751, Feb. 1994.

[8] S. Vishawanath, N. Jindal, and A. J. Goldsmith, "Duality, achievable rates, and sum-rate capacity of Gaussian MIMO broadcast channels," IEEE Trans. Info. Theory, vol. 49, no. 10, pp. 2658-2668, Oct. 2003.

[9] T. L. Marzetta, "Noncooperative cellular wireless with unlimited numbers of base station antennas," IEEE Trans. Wireless Commun., vol. 9, no. 11, pp. 3590-3600, Nov. 2010. 
[10] D. Gesbert, M. Kountouris, R. W. Heath Jr., C.-B. Chae, and T. Salzer, "Shifting the MIMO paradigm," IEEE Signal Process. Mag., vol. 24, no. 5, pp. 36-46, Sept. 2007.

[11] E. G. Larsson, O. Edfors, F. Tufvesson, and T. L. Marzetta, "Massive MIMO for next generation wireless systems," IEEE Commun. Mag., vol. 52, no. 2, pp. 186-195, Feb. 2014.

[12] T. S. Rappaport, S. Sun, R. Mayzus, H. Zhao, Y. Azar, K. Wang, G. N. Wong, J. K. Schulz, M. K. Samimi, and F. Gutierrez, "Millimeter wave mobile communications for $5 \mathrm{G}$ cellular: It will work!," IEEE Access, vol. 1, pp. 335-349, Apr. 2013.

[13] A. L. Swindlehurst, E. Ayanoglu, P. Heydari, and F. Capolino, "Millimeter-wave massive MIMO: The next wireless revolution?," IEEE Commun. Mag., vol. 52, no. 9, pp. 56-62, Sep. 2014.

[14] A. F. Molisch, Wireless Communications, Wiley Press, 2011.

[15] A. J. Goldsmith, Wireless Communications, vol. 2, Cambridge Press, 2005.

[16] J. G. Proakis, Digital Communications, McGraw-Hill, 2001.

[17] S. Verdú, Multiuser Detection, Cambridge University Press, 1998.

[18] R. A. Horn and C. A. Johnson, Matrix Analysis, Cambridge University Press, 1985.

[19] R. J. Muirhead, Aspect of Multivariate Statistical Theory, John Wiley, 1982.

[20] N. R. Goodman, "Statistical analysis based on certain multivariate complex distribution," Annals of Mathematical Sciences, vol. 34, no. 1, pp. 152-177, Apr. 1963. 
[21] D. S. Shiu, G. J. Foschini, M. J. Gans, and J. M. Kahn, "Fading correlation and its effect on the capacity of multielement antenna systems," IEEE Trans. Commun., vol. 48, no. 3, pp. 502-513, Mar. 2000.

[22] P. J. Smith, L. M. Garth, and S. L. Loyka, "Exact capacity distributions for MIMO systems with small numbers of antennas," IEEE Commun. Lett., vol. 7, no. 10, pp. 481-483, Oct. 2003.

[23] P. J. Smith and L. M. Garth, "Exact capacity distribution for dual MIMO systems in Ricean fading," IEEE Commun. Lett., vol. 8, no. 1, pp. 18-20, Jan. 2004.

[24] P. F. Driessen and G. J. Foschini, "On the capacity formula for multiple input-multiple output wireless channels: A geometric interpretation," in Proc. Int. Conf. on Commun. (ICC), Jun. 2009, pp. 1603-1607.

[25] S. Catreux, P. F. Driessen, and L. J. Greenstein, "Attainable throughput of an interference-limited multiple-input multiple-output (MIMO) cellular system," IEEE Trans. Commun., vol. 49, no. 8, pp. 1307-1311, Aug. 2001.

[26] H. Dai, A. F. Molisch, and H. V. Poor, "Downlink capacity of interferencelimited MIMO systems with joint detection," IEEE Trans. Wireless Commun., vol. 3, no. 2, pp. 442-453, Mar. 2004.

[27] B. Skalar, "Rayleigh fading channels in mobile digital communication systems part I: Charaterization," IEEE Commun. Mag., vol. 35, no. 9, pp. 136-146, Jul. 1997.

[28] A. A. M. Saleh and R. A. Valenzuela, "A statistical model for indoor multipath propagation," IEEE J. Sel. Topics in Commun., vol. 5, no. 2, pp. 128-137, Feb. 1987.

[29] P. A. Bello, "Characterization of randomly time-variant linear channels," IEEE Trans. Commun., vol. 11, pp. 360-393, Dec. 1963. 
[30] T. S. Rappaport, Wireless Communications: Principles and Practice, Prentice-Hall, 2001.

[31] C. Oestges and B. Clerckx, MIMO Wireless Communications: From RealWorld Propagation to Space-Time Code Design, Academic Press, 2007.

[32] M. Steinbauer, A. F. Molisch, and E. Bonek, "The double-directional radio channel," IEEE Antennas and Propag. Mag., vol. 43, no. 4, pp. 51-63, Aug. 2001.

[33] J. P. Kermoal, L. Schumacher, K. Pedersen, P. E. Mogensen, and F. Frederiksen, "A stochastic MIMO radio channel model with experimental validation," IEEE J. Sel. Areas Commun., vol. 20, no. 6, pp. 1211-1226, Aug. 2002.

[34] P. J. Smith and M. Shafi, "The impact of complexity in MIMO channel models," in Proc. IEEE Int. Conf. on Commun. (ICC), Jun. 2004, pp. 2924-2928.

[35] K. Haneda et al., "Indoor 5G 3GPP-like channel models for office and shopping mall environments," in Proc. IEEE Int. Conf. on Commun. (ICC) Workshops, May 2016, pp. 1-6.

[36] D. N. C. Tse and P. Viswanath, Fundamentals of Wireless Communication, Cambridge University Press, 2005.

[37] A. Adhikary, A. Ashikhmin, and T. L. Marzetta, "Uplink interference reduction in large scale antenna systems," IEEE Trans. Commun., vol. PP, no. 99, pp. 1-16, Jan. 2017.

[38] L. Zheng and D. N. C. Tse, "Diversity and multiplexing: A fundamental tradeoff in multiple-antenna channels," IEEE Trans. Info. Theory, vol. 49, no. 5, pp. 1073-1096, May 2003.

[39] J. D. Parsons, The mobile radio propagation channel, Wiley Publishers, 2000. 
[40] J. Fuhl, A. F. Molisch, and E. Bonek, "Unified channel model for mobile radio systems with smart antennas," in Proc. IEE Radar and Sonar Navigation, vol. 145, pp. 32-41, 1998.

[41] J. Nam, G. Caire, and J. Ha, "On the role of transmit correlation diversity in multiuser MIMO systems," IEEE Trans. Info. Theory, vol. 63, no. 1, pp. 336-354, Jan. 2017.

[42] J. Nam, J. Y. Ahn, A. Adhikary, and G. Caire, "Joint spatial division and multiplexing: Realizing massive MIMO gains with limited channel state information," in Proc. Conf. Inf. Sci. Systems (CISS), Mar. 2012, pp. 1-6.

[43] A. Adhikary, J. Nam, J.-Y. Ahn, and G. Caire, "Joint spatial division and multiplexing - The large-scale array regime," IEEE Trans. Info. Theory, vol. 59, no. 10, pp. 6441-6463, Oct. 2013.

[44] A. Adhikary, E. Al. Safadi, M. K. Samimi, R. Wang, G. Caire, T. S. Rappaport, and A. F. Molisch, "Joint spatial division and multiplexing for mm-Wave channels," IEEE J. Sel. Areas Commun., vol. 32, no. 6, pp. 1239-1255, Jun. 2014.

[45] D. Chizhik, F. Farrokhi, J. Ling, and A. Lozano, "Effect of antenna separation on the capacity of BLAST in correlated channels," IEEE Commun. Lett., vol. 4, no. 12, pp. 337-339, Nov. 2000.

[46] S. L. Loyka, "Channel capacity of MIMO architecture using the exponential correlation matrix," IEEE Commun. Lett., vol. 5, no. 9, pp. 369-371, Sep. 2001.

[47] H. Shin and J. H. Lee, "Capacity of multiple-antenna fading channels: Spatial fading correlation, double scattering, and keyhole," IEEE Trans. Info. Theory, vol. 49, no. 10, pp. 2636-2647, Oct. 2003.

[48] W. C. Jakes, Microwave Mobile Communications, IEEE, Jan. 1974. 
[49] F. Farrokhi, G. J. Foschini, A. Lozano, and R. A. Valenzuela, "Linkoptimal space-time processing with multiple transmit and receive antennas," IEEE Commun. Lett., vol. 5, no. 3, pp. 85-87, Mar. 2001.

[50] A. Zanella, M. Chiani, and M. Z. Win, "On the marginal distribution of the eigenvalues of Wishart matrices," IEEE Trans. Commun., vol. 57, no. 4, pp. 1050-1060, Apr. 2009.

[51] M. Matthaiou, M. R. McKay, P. J. Smith, and J. A. Nossek, "On the condition number distribution of complex Wishart matrices," IEEE Trans. Commun., vol. 58, no. 6, pp. 1705-1717, Jun. 2010.

[52] J. Wishart, "The generalised product moment distribution in samples from a normal multivariate population," Biometrika, vol. 20, pp. 32-52, 1928.

[53] J. Wishart, "Proofs of the distribution law of the second order moment statistics," Biometrika, vol. 35, pp. 475-501, 1948.

[54] A. T. James, "Distributions of matrix variates and latent roots derived from normal samples," Annals of Mathematics and Statistics, vol. 35, pp. 475-501, 1964.

[55] P. J. Smith and M. Shafi, "An approximate capacity distribution for MIMO systems," IEEE Trans. Commun., vol. 52, no. 6, pp. 887-890, Jun. 2004.

[56] H. Shin, M. Z. Win, J. H. Lee, and M. Chiani, "On the capacity of doubly correlated MIMO channels," IEEE Trans. Wireless Commun., vol. 5, no. 8, pp. 2253-2265, Aug. 2006.

[57] F. Rusek, D. Persson, B. Lau, E. G. Larsson, T. L. Marzetta, O. Edfors, and F. Tufvesson, "Scaling up MIMO: Opportunities and challenges with very large arrays," IEEE Signal Process. Mag., vol. 30, no. 1, pp. 40-60, Jan. 2013. 
[58] P. J. Smith, C. T. Neil, M. Shafi, and P. A. Dmochowski, "On the convergence of massive MIMO systems," in IEEE Int. Conf. on Commun. (ICC), Jun. 2014, pp. 5191-5196.

[59] H. Q. Ngo, E. G. Larsson, and T. L. Marzetta, "The multicell multiuser MIMO uplink with very large antenna arrays and a finite-dimensional channel," IEEE Trans. Commun., vol. 6, no. 61, pp. 2350-2361, Jun. 2013.

[60] J. Hoydis, S. ten Brink, and M. Debbah, "Massive MIMO in the UL/DL of cellular networks: How many antennas do we need?," IEEE J. on Sel. Areas Commun., vol. 31, no. 2, pp. 160-171, Feb. 2013.

[61] J. Hoydis, S. ten Brink, and M. Debbah, "Comparison of linear precoding schemes for downlink massive MIMO," in Proc. IEEE Int. Conf. on Commun. (ICC), Jun. 2012, pp. 2135-2139.

[62] A. M. Tulino and S. Verdú, "Random matrix theory and wireless communications," Foundations Trends Commun. Info. Theory, vol. 1, no. 1, pp. 1-182, Jun. 2004.

[63] I. Gradshteyn and I. Ryzhik, Table of Integrals, Series, and Products, Academic Press, 2007.

[64] G. Alfano, A. M. Tulino, A. Lozano, and S. Verdú, "Capacity of MIMO channels with one-sided correlation," in IEEE Eight Int. Symp. on Spread Spectrum Tech. and App. (ISSSTA), Aug. 2004, pp. 515-519.

[65] P. J. Smith, S. Roy, and M. Shafi, "Capacity of MIMO systems with semicorrelated flat fading," IEEE Trans. Info. Theory., vol. 49, no. 10, pp. 2781-2788, Oct. 2003.

[66] G. Alfano, A. Lozano, A. M. Tulino, and S. Verdú, "Mutual information and eigenvalue distribution of MIMO Ricean channels," in Int. Symposium on Info. Theory and Applications (ISITA), Oct. 2004, pp. 1-6. 
[67] C. Kong, C. Zhong, M. Matthaiou, and Z. Zhang, "Performance of downlink massive MIMO in Ricean fading channels with ZF precoder," in IEEE Int. Conf. on Commun. (ICC), Jun. 2015, pp. 1776-1782.

[68] Q. Zhang, S. Jin, K.-K. Wong, H. Zhu, and M. Matthaiou, "Power scaling of uplink massive MIMO systems with arbitrary-rank channel means," IEEE J. Sel. Topics Signal Process., vol. 8, no. 5, pp. 966-981, Oct. 2014.

[69] F. Boccardi, R. W. Heath Jr., A. Lozano, T. L. Marzetta, and P. Popovski, "Five disruptive technology directions for 5G," IEEE Commun. Mag., vol. 52, no. 2, pp. 74-80, Feb. 2014.

[70] J. G. Andrews, S. Buzzi, W. Choi, S. V. Hanly, A. Lozano, A. C. K. Soong, and J. C. Zhang, "What will 5G be?," IEEE J. Sel. Areas in Commun., vol. 32, no. 6, pp. 1065-1082, Mar. 2014.

[71] T. L. Marzetta and B. M. Hochwald, "Capacity of a mobile multipleantenna communication link in Rayleigh flat fading," IEEE Trans. Info. Thoery, vol. 45, no. 1, pp. 139-157, Jan. 1999.

[72] A. J. Goldsmith, S. A. Jafar, N. Jindal, and S. Vishawanath, "Capacity limits of MIMO channels," IEEE J. Sel. Areas Commun., vol. 21, no. 5, pp. 684-702, Jun. 2003.

[73] T. L. Marzetta, "Massive MIMO: An introduction," Bell Systems Tech. Journal, vol. 20, no. 1, pp. 11-22, Mar. 2015.

[74] X. Gao, O. Edfors, F. Rusek, and F. Tufvesson, "Linear pre-coding performance in measured very-large MIMO channels," in Proc. IEEE Veh. Technol. Conf. (VTC-Fall), Sept. 2011, pp. 1-5.

[75] S. L. H. Nguyen, K. Haneda, J. Jarvelainen, A. Karttunen, and J. Putkonen, "On the mutual orthogonality of millimeter-wave massive MIMO channels," in IEEE Veh. Technol. Conf. (VTC-Spring, May 2015, pp. $1-5$. 
[76] K. A. Alnajjar, Receiver Design for Massive MIMO, Ph.D. Thesis, University of Canterbury, New Zealand, 2015.

[77] A. Kammoun, A. Muller, E. Björnson, and M. Debbah, "Linear precoding based on polynomial expansion: Large-scale multi-cell MIMO systems," IEEE Journal on Sel. Topics in Signal Process., vol. 8, no. 5, pp. 861-875, Oct. 2014.

[78] D. Zhu, B. Li, and P. Liang, "On the matrix inversion approximation based on Neumann series in massive MIMO systems," in IEEE Int. Conf. on Commun. (ICC), Nov. 2008, pp. 1-5.

[79] E. Björnson, E. G. Larsson, and T. L. Marzetta, "Massive MIMO: Ten myths and one critical question," IEEE Commun. Mag., vol. 54, no. 2, pp. 114-123, Feb. 2016.

[80] H. Tataria, P. J. Smith, P. A. Dmochowski, and M. Shafi, "General analysis of multiuser MIMO systems with regularized zero-forcing precoding under spatially correlated Rayleigh fading channels," in Proc. of IEEE Int. Conf. on Commun. (ICC), May 2016, pp. 2582-2588.

[81] H. Tataria, P. J. Smith, M. Matthaiou, and P. A. Dmochowski, "Uplink analysis of large MU-MIMO systems with space-constrained arrays in Ricean fading," in Proc. of IEEE Int. Conf. on Commun. (ICC), May 2017, pp. 1-7.

[82] H. Tataria, P. J. Smith, L. J. Greenstein, and P. A. Dmochowski, "Zeroforcing precoding performance in multiuser MIMO systems with heterogeneous Ricean fading," IEEE Wireless Commun. Lett., vol. 6, no. 1, pp. 74-77, 2017.

[83] H. Tataria, M. Shafi, P. J. Smith, and P. A. Dmochowski, "Coordinated two-tier heterogeneous cellular networks with leakage based beamforming," CoRR. vol.abs/1503.01566, Mar. 2015. 
[84] H. Tataria, P. J. Smith, L. J. Greenstein, P. A. Dmochowski, and M. Shafi, "Performance and analysis of downlink multiuser MIMO systems with regularized zero-forcing precoding in Ricean fading channels," in Proc. of IEEE Int. Conf. on Commun. (ICC), May 2016, pp. 1185-1192.

[85] H. Tataria, P. J. Smith, and P. A. Dmochowski, "On the general analysis of coordinated regularized zero-forcing precoding: An application to twotier small-cell networks," Under Revisions for Publication in IEEE Trans. Commun., pp. 1-15, 2016.

[86] D. Basnayaka, P. J. Smith, and P. A. Martin, "Performance analysis of macrodiversity MIMO systems with MMSE and ZF receivers in flat Rayleigh fading," IEEE Trans. Wireless Commun., vol. 12, no. 5, pp. 2240-2251, May 2013.

[87] D. Basnayaka, P. J. Smith, and P. A. Martin, "The effect of macrodiversity on the performance of maximal ratio combining in flat Rayleigh fading," IEEE Trans. Commun., vol. 61, no. 4, pp. 1384-1392, Apr. 2013.

[88] O. Lieberman, "A Laplace approximation to the moments of a ratio of quadratic forms," Biometrika, vol. 81, no. 4, pp. 681-690, Dec. 1994.

[89] C. Masouros, M. Sellathurai, and T. Ratnarajah, "Large-scale MIMO transmitters in fixed physical spaces: The effect of transmit correlation and mutual coupling," IEEE Trans. Commun., vol. 61, no. 7, pp. 27942804, Jul. 2013.

[90] C. Masouros and M. Matthaiou, "Space-constrained massive MIMO: Hitting the wall of favorable propagation," IEEE Commun. Lett., vol. 19, no. 5, pp. 771-774, May 2015.

[91] A. Garcia-Rodriguez and C. Masouros, "Exploiting the increasing correlation of space constrained massive MIMO for CSI relaxation," IEEE Trans. Commun., vol. 4, no. 64, pp. 1572-1587, Apr. 2016. 
[92] S. Biswas, C. Masouros, and T. Ratnarajah, "Performance analysis of large multi-user MIMO systems with space-constrained 2D antenna arrays," IEEE Trans. Wireless Commun., vol. 15, no. 5, pp. 3492-3505, May 2016.

[93] J. Zhang, L. Dai, M. Matthaiou, C. Masouros, and S. Jin, "On the spectral efficiency of space-constrained massive MIMO with linear receivers," in Proc. of IEEE Int. Conf. on Commun. (ICC), May 2016, pp. 1-6.

[94] A. Li and C. Masouros, "Exploiting constructive mutual coupling in P2P MIMO by analog-digital phase alignment," IEEE Trans. Wireless Commun., vol. 16, no. 3, pp. 1948-1962, Mar. 2017.

[95] A. Lozano, R. W. Heath Jr., and J. G. Andrews, "Fundamental limits of cooperation," IEEE Trans. Info. Theory, vol. 59, no. 9, pp. 5213-5226, Sep. 2013.

[96] J. Zhang, R. Chen, J. G. Andrews, A. Ghosh, and R. W. Heath Jr., "Networked MIMO with clustered linear precoding," IEEE Trans. Wireless Commun., vol. 8, no. 4, pp. 1910-1921, Apr. 2009.

[97] C.-B. Chae, S.-H. Kim, and R. W. Heath Jr., "Network coordinated beamforming for cell-boundary users: Linear and nonlinear approaches," IEEE J. Sel. Topics Signal Process., vol. 3, no. 6, pp. 1094-1105, Dec. 2009.

[98] D. Gesbert, S. V. Hanly, H. Huang, S. Shamai, S. Osvaldo, and Y. Wei, "Multi-cell MIMO cooperative networks: A new look at interference," IEEE J. Sel. Areas Commun., vol. 28, no. 9, pp. 1380-1408, Dec. 2010.

[99] B. Soret, K. Pedersen, N. Jrgensen, and V. Fernández-Loṕez, "Interference coordination for dense wireless networks," IEEE Commun. Mag., vol. 53, no. 1, pp. 102-109, Jan. 2015. 
[100] G. Nigam, P. Minero, and M. Haenggi, "Coordinated multipoint joint transmission in heterogeneous networks," IEEE Trans. Wireless Commun., vol. 62, no. 11, pp. 4134-4146, Jun. 2014.

[101] H. Kim, H. Yu, and Y. Lee, "Limited feedback for multicell zero-forcing coordinated beamforming in time-varying channels," IEEE Trans. Veh. Technol., vol. 64, no. 6, pp. 2349-2359, Jun. 2015.

[102] S. Lakshminarayana, M. Assad, and M. Debbah, "Coordinated multicell beamforming for massive MIMO: A random matrix approach," IEEE Trans. Info. Theory, vol. 61, no. 6, pp. 3387-3412, Jun. 2015.

[103] H. Huang, M. Trivellato, A. Hottinen, M. Shafi, P. J. Smith, and R. A. Valenzuela, "Increasing downlink cellular throughput with limited network MIMO coordination," IEEE Trans. Wireless Commun., vol. 8, no. 6, pp. 2983-2989, Jun. 2009.

[104] R. Muharar, R. Zakhour, and J. Evans, "Base station cooperation with feedback optimization: A large system analysis," IEEE Trans. Info. Theory, vol. 60, no. 6, pp. 3620-3644, Jun. 2014.

[105] O. Simeone, O. Somekh, H. V. Poor, and S. Shamai, "Local base station cooperation via finite-capacity links for the uplink of linear cellular networks," IEEE Trans. Info. Theory, vol. 55, no. 1, pp. 190-204, Jan. 2009.

[106] R. Irmer, H. Droste, P. Marsch, M. Grieger, G. Fettweis, S. Brueck, H.-P Mayer, L. Thiele, and V. Jungnickel, "Coordinated multipoint: Concepts, performance, and field trails," IEEE Commun. Mag., vol. 49, no. 2, pp. 102-111, Feb. 2011.

[107] D. Lee, H. Seo, B. Clerckx, E. Hardouin, E. Mazzarese, S. Nagata, and K. Sayana, "Coordinated multipoint transmission and reception in LTEAdvanced: Deployment scenarios and operational challenges," IEEE Commun. Mag., vol. 50, no. 2, pp. 148-155, Feb. 2012. 
[108] 3GPP TR 36.847 v.12.0.0, Study on LTE Time Division Duplex (TDD)Frequency Division Duplex (FDD) joint operation including Carrier Aggregation (CA), 3GPP, Dec. 2013.

[109] S. Sun, T. S. Rappaport, R. W. Heath Jr., A. R. Nix, and S. Rangan, "MIMO for millimeter-wave wireless communications: Beamforming, spatial multiplexing, or both?," IEEE Commun. Mag., vol. 52, no. 12, pp. 110-121, Dec. 2014.

[110] J. Fan, Z. Xu, and G. Li, "Performance analysis of MU-MIMO in downlink cellular networks," IEEE Commun. Lett., vol. 19, no. 2, pp. 223-226, Feb. 2015.

[111] H. Falconet, L. Sanguinetti, A. Kammoun, and M. Debbah, "Asymptotic analysis of downlink MISO systems over Rician fading channels," in IEEE Int. Conf. on Acoustics, Speech and Signal Process. (ICASSP), Mar. 2016, pp. 3926-3930.

[112] J. Choi, "Distributed beamforming for macro diversity and power control with large arrays in spatial correlated channels," IEEE Trans. Wireless Commun., vol. 14, no. 4, pp. 1817-1881, Apr. 2015.

[113] H. S. Dhillon, R. K. Ganti, F. Baccelli, and J. G. Andrews, "Modeling and analysis of K-tier downlink heterogeneous cellular networks," IEEE J. Sel. Areas Commun., vol. 30, no. 3, pp. 550-560, Mar. 2012.

[114] H. S. Dhillon, R. K. Ganti, and J. G. Andrews, "Load-aware modeling and analysis of heterogeneous cellular networks," IEEE Trans. Wireless Commun., vol. 12, no. 4, pp. 1666-1677, Apr. 2013.

[115] H. S. Dhillon, M. Kountouris, and J. G. Andrews, "Downlink MIMO hetnets: Modeling, ordering results and performance analysis," IEEE Trans. Wireless Commun., vol. 12, no. 10, pp. 5208-5222, Oct. 2013.

[116] S. Wagner, R. Couillet, M. Debbah, and D. T. M. Slock, "Large system analysis of linear precoding in correlated MISO broadcast channels under 
limited feedback," IEEE Trans. Info. Theory, vol. 58, no. 7, pp. 45094537, Dec. 2012.

[117] Z. Jiang, A. F.Molisch, G. Caire, and Z. Niu, "Achievable rates of FDD massive MIMO systems with spatial channel correlation," IEEE Trans. Wireless Commun., vol. 14, no. 5, pp. 2868-2882, May 2015.

[118] C. B. Peel, B. M. Hochwald, and A. L. Swindlehurst, "A vector-perturbation technique for near-capacity multiantenna multiuser communication-part I: Channel inversion and regularization," IEEE Trans. Commun., vol. 53, no. 1, pp. 195-202, Jan. 2005.

[119] D. Hwang, B. Clerckx, and G. Kim, "Regularized channel inversion with quantized feedback in down-link multiuser channels," IEEE Trans. Wireless Commun., vol. 8, no. 12, pp. 5785-5789, Dec. 2009.

[120] B. Clerckx, G. Kim, and S. Kim, "Correlated fading in broadcast MIMO channels: Curse or blessing?," in Proc. IEEE Global Commun. Conf. (GLOBECOM), Nov. 2008, pp. 1-5.

[121] B. Clerckx, G. Kim, and S. Kim, "MU-MIMO with channel statistics based codebooks in spatially correlated channels," in Proc. of IEEE Global Commun. Conf. (GLOBECOM), Nov. 2008, pp. 1-5.

[122] D. N Nguyen and T. Le-Ngoc, "MMSE precoding for multiuser MISO downlink transmission with non-homogeneous user SNR conditions," EURASIP Journal on Adv. Signal Process., vol. 85, no. 1, pp. 1-12, Jun. 2014.

[123] T. M. Cover and J. A. Thomas, Elements of Information Theory, vol. 3, John Wiley Sons, 1991.

[124] H. Gao, P. J. Smith, and M. V. Clark, "Theoretical reliability of MMSE linear diversity combining in Rayleigh-fading additive interference channels," IEEE Trans. Commun., vol. 46, no. 5, pp. 666-672, May 1998. 
[125] 3GPP TR 36.814 v.9.0.0, Further advancements for E-UTRA physical layer aspects, 3GPP, Jun. 2010.

[126] T. S. Rappaport, F. Gutierrez, E. Ben-Dor, J. N. Murdock, Y. Qaio, and J. I. Tamir, "Broadband millimeter-wave propagation measurements and models using adaptive-beam antennas for outdoor urban cellular communications," IEEE Trans. Antennas and Propag., vol. 61, no. 12, pp. 1850-1859, Dec. 2012.

[127] T. S. Rappaport, G. R. MacCartney, M. K. Samimi, and S. Sun, "Wideband millimeter-wave propagation measurements and channel models for future wireless communications system design," IEEE Trans. Commun., vol. 63, no. 5, pp. 3029-3056, May 2015.

[128] M. K. Samimi and T. S. Rappaport, "3-D millimeter-wave statistical channel model for 5G wireless system design," IEEE Trans. Microwave Theory and Tech., vol. 64, no. 7, pp. 2207-2225, Jul. 2016.

[129] T. Thomas, H. C. Nugyen, G. R. MacCartney, and T. S. Rappaport, “3D mmWave channel model proposal," in Proc. IEEE Veh. Technol. Conf. (VTC-Fall), Sep. 2014, pp. 1-6.

[130] S. Hur, S. Baek, B. Kim, Y. Chang, A. F. Molisch, T. S. Rappaport, K. Haneda, and J. Park, "Proposal on millimeter-wave channel modeling for 5G cellular system," IEEE J. Sel. Topics Signal Process., vol. 10, no. 3, pp. 454-469, Apr. 2016.

[131] M. K. Samimi and T. S. Rappaport, "3-D statistical channel model for millimeter-wave outdoor mobile broadband communications," in IEEE Int. Conf. on Commun. (ICC), Jun. 2015, pp. 2430-2436.

[132] M. K. Samimi and T. S. Rappaport, "3-D millimeter-wave statistical channel model for 5G wireless system design," IEEE Trans. Microwave Theory Techn., vol. 64, no. 7, pp. 2207-2225, Jul. 2016. 
[133] M. K. Samimi and T. S. Rappaport, "Statistical channel model with multifrequency and arbitrary antenna beamwidth for millimeter-wave outdoor communications," in IEEE Global Conf. on Commun. (GLOBECOM), Dec. 2015, pp. 1-7.

[134] W. Tan, S. Jin, J. Wang, and M. Matthaiou, "Achievable sum-rate of multiuser massive MIMO downlink in Ricean fading channels," in IEEE Int. Conf. on Commun. (ICC), Jun. 2015, pp. 1453-1458.

[135] S. Jin, W. Tan, M. Matthaiou, J. Wang, and K.-K. Wong, "Statistical eigenmode transmission for the MU-MIMO downlink in Rician fading," IEEE Trans. Wireless Commun., vol. 14, no. 12, pp. 6650-6663, Dec. 2015 .

[136] 3GPP TR 36.873 v.12.2.0, Study on 3D channel models for LTE, 3GPP, Jun. 2015.

[137] M. R. Akdeniz, Y. Liu, M. K. Samimi, S. Sun, S. Rangan, T. S. Rappaport, and E. Erkip, "Millimeter wave channel modeling and cellular capacity evaluation," IEEE J. Sel. Areas Commun., vol. 32, no. 6, pp. 1164-1179, Jun. 2014.

[138] J. Zhang, L. Dai, S. Sun, and Z. Wang, "On the spectral efficiency of massive MIMO systems with low-resolution ADCs," IEEE Commun. Lett., vol. 20, no. 5, pp. 842-845, May 2016.

[139] J. Mirza, P. J. Smith, and P. A. Dmochowski, "Coordinated regularized zero-forcing precoding for multicell MISO systems with limited feedback," IEEE Trans. Veh. Technol., vol. 66, no. 1, pp. 335-343, Jan. 2017.

[140] D. Gore, R. W. Heath Jr., and A. J. Paulraj, "Transmit selection in spatial multiplexing systems," IEEE Commun. Lett., vol. 6, no. 11, pp. 491-493, Nov. 2002. 
[141] P. Li, D. Paul, R. Narasimhan, and J. Cioffi, "On the distribution of SINR for the MMSE MIMO receiver and performance analysis," IEEE Trans. Info. Theory, vol. 52, no. 1, pp. 271-286, Jan. 2006.

[142] R. H. Y. Louie, M. R. McKay, and I. B. Collings, "New performance results for multiuser optimum combining in the presence of Rician fading," IEEE Trans. Commun., vol. 57, no. 8, pp. 2348-2358, Aug. 2009.

[143] Z. Lin, T. Sorensen, and P. E. Mogensen, "Downlink SINR distribution of linearly precoded multiuser MIMO systems," IEEE Commun. Lett., vol. 11, no. 11, pp. 850-853, Nov. 2007.

[144] H. Q. Ngo, M. Matthaiou, T. Q. Duong, and E. G. Larsson, "Uplink performance analysis of multicell MU-SIMO systems with ZF receivers," IEEE Trans. Veh. Technol., vol. 62, no. 9, pp. 4471-4483, Nov. 2013.

[145] H. Q. Ngo, T. Q. Duong, and E. G. Larsson, "Uplink performance analysis of multicell MU-MIMO with zero-forcing receivers and perfect CSI," in IEEE Swedish Communication Technologies Workshop (Swe-CTW), Oct. 2011, pp. 40-45.

[146] H. Yang and T. L. Marzetta, "Performance of conjugate and zero-forcing beamforming in large-scale antenna systems," IEEE J. Sel. Areas Commun., vol. 31, no. 2, pp. 172-179, Feb. 2013.

[147] N. Johnson and S. Kotz, Continuous Univariate Distributions, vol. 2, Wiley Publishers, 1994.

[148] A. Mehana and A. Nosratinia, "Diversity of MIMO linear precoding," IEEE Trans. Info. Theory, vol. 60, no. 2, pp. 1019-1038, Feb. 2014.

[149] R. Senanayake, P. J. Smith, P. L. Yeoh, and J. Evans, "An SNR approximation for distributed massive MIMO with zero forcing," IEEE Commun. Lett., vol. 19, no. 11, pp. 1885-1888, Nov. 2015. 
[150] K. A. Alnajjar, P. J. Smith, G. K. Woodward, and D. A. Basnayaka, "Design and analysis of a reduced complexity MRC V-BLAST receiver for massive MIMO," in IEEE Int. Workshop Signal Process. Advanced Wireless Commun. (SPAWC), July 2016, pp. 1-6.

[151] B. Wang, Y. Chang, and D. Yang, "On the SINR in massive MIMO networks with MMSE receivers," IEEE Commun. Lett., vol. 18, no. 11, pp. 1979-1982, Nov. 2014.

[152] K. Biyari and W. Lindsey, "Statistical distributions of Hermitian quadratic forms in complex Gaussian variables," IEEE Trans. Info. Theory, vol. 39, no. 3, pp. 1076-1082, May 1993.

[153] O. El Ayach, S. Rajagopal, S. Abu-Surra, and R. W. Heath Jr., "Spatially sparse precoding in millimeter wave MIMO systems," IEEE Trans. Wireless Commun., vol. 13, no. 3, pp. 1499-1513, Mar. 2014.

[154] C. N. Chuah, D. N. C. Tse, J. M. Kahn, and R. A. Valenzuela, "Capacity scaling in MIMO wireless systems under correlated fading," IEEE Trans. Info. Theory, vol. 48, no. 3, pp. 637-650, Mar. 2002.

[155] V. V. Veeravalli, Y. Liang, and A. M. Sayeed, "Correlated MIMO wireless channels: Capacity, optimal signaling and asymptotics," IEEE Trans. Info. Theory, vol. 51, no. 6, pp. 2058-2072, Jun. 2005.

[156] A. M. Tulino, A. Lozano, and S. Verdú, "Impact of antenna correlation on the capacity of multiantenna channels," IEEE Trans. Info. Theory, vol. 51, no. 7, pp. 2491-2509, 2005.

[157] S. A. Jafar and A. J. Goldsmith, "Multiple-antenna capacity in correlated Rayleigh fading with channel covariance information," IEEE Trans. Wireless Commun., vol. 4, no. 3, pp. 990-997, May 2005.

[158] P. T. Samarasinghe, Mobile to Mobile Channel Modelling for Wireless Communications, Ph.D. Thesis, Australian National University, 2013. 
[159] X. Ge, R. Zi, H. Wang, J. Zhang, and M. Jo, "Multi-user massive MIMO communication systems based on irregular antenna arrays," IEEE Trans. Wireless Commun., vol. 15, no. 8, pp. 5287-5301, Aug. 2016.

[160] D. Hammarwall, M. Bengtsson, and B. Ottersten, "Utilizing the spatial information provided by channel norm feedback in SDMA system," IEEE Trans. Signal Process., vol. 56, no. 7, pp. 3278-3293, Jul. 2008.

[161] F. Boccardi M. Trivellato and H. Huang, "On transceiver design and channel quantization for downlink multiuser MIMO systems with limited feedback," IEEE Journal on Sel. Areas in Commun., vol. 26, no. 8, pp. 1494-1504, Oct. 2008.

[162] J. Y. Ko J. W. Lee and Y. H. Lee, "Effect of transmit correlation on the sum-rate capacity of two-user broadcast channels," IEEE Trans. Commun., vol. 57, no. 9, pp. 2597-2599, Sep. 2009.

[163] J. Nam and J. Y. Ahn, "Joint spatial division and multiplexing-Benefits of antenna correlation in multi-user MIMO," in Proc. IEEE Int. Symp. Info. Theory (ISIT), Jul. 2013, pp. 619-623.

[164] C. Gustafson, K. Haneda, S. Wyne, and F. Tufvesson, "On mm-wave multipath clustering and channel modeling," IEEE Trans. Antennas and Prop., vol. 62, no. 3, pp. 1445-1455, Mar. 2014.

[165] A. F. Molisch, Signal Processing for 5G: Algorithms and Implementations - 3D Propagation Channels: Modeling and Measurements, Wiley Publishers, 2016.

[166] E. Björnson, J. Hoydis, and L. Sanguinetti, "Pilot contamination is not a fundamental asymptotic limitation in massive MIMO," CoRR. vol.abs/1611.09152, Nov. 2016.

[167] A. Ashikhmin and T. L. Marzetta, "Pilot contamination precoding in multi-cell large scale antenna systems," in IEEE Int. Symp. on Info. Theory (ISIT), Jul. 2012, pp. 1137-1141. 
[168] M. Matthaiou E. Björnson and M. Debbah, "Massive MIMO with nonideal arbitrary arrays: Hardware scaling laws and circuit-aware design," IEEE Trans. Wireless Commun., vol. 14, no. 8, pp. 4353-4368, Apr. 2015.

[169] A. Forenza, D. J. Love, and R. W. Heath, "Simplified spatial correlation models for clustered MIMO channels with different array configurations," IEEE Trans. Veh. Technol., vol. 56, no. 4, pp. 1924-1934, Jul. 2007.

[170] G. Y. Li, Z. Xu, C. Yang, S. Zhang Y. Chen, and S. Xu, "Energy-efficient wireless communications: Tutorial, survey, and open issues," IEEE Wireless Commun., vol. 18, no. 6, pp. 28-35, Dec. 2011.

[171] V. Raghavan and A. M. Sayeed, "Sublinear capacity scaling laws for sparse MIMO channels," IEEE Trans. Info. Theory, vol. 57, no. 1, pp. 345-364, 2011.

[172] H. Wei, H. Zhu, J. Wang, S. Sun, and X. You, "Mutual coupling calibration for multiuser massive MIMO systems," IEEE Trans. Wireless Commun., vol. 15, no. 1, pp. 606-619, Jan. 2016.

[173] B. Clerckx, C. Craeye, D. Vanhoenacker-Janvier, and C. Oestges, "Impact of antenna coupling on 2x2 MIMO communications," IEEE Trans. Veh. Technol., vol. 56, no. 3, pp. 1009-1018, May 2007.

[174] K. Warnick and M. Jensen, "Optimal noise matching for mutually coupled arrays," IEEE Trans. Antennas Propag., vol. 55, no. 6, pp. 1726-1731, Jun. 2007.

[175] A. Karttunen, A. F. Molisch, S. Hur, J. Zhang, and J. Park, "Distance dependence of path loss models with weighted fitting," in Proc. IEEE Int. Conf. on Commun. (ICC), Jul. 2016, pp. 1-6.

[176] A. F. Molisch and F. Tufvesson, "Propagation channel models for nextgeneration wireless communication systems," IEICE Trans. Commun., vol. E97-B, no. 10, pp. 2022-2034, Oct. 2014. 
[177] 3GPP TR 38.900 v.14.2.0, Study on channel model for frequency spectrum above $6 \mathrm{GHz}$, 3GPP, Jan. 2017.

[178] H. Yin, D. Gesbert, M. Filippou, and Y. Liu, "A coordinated approach to channel estimation in large-scale multiple-antenna systems," IEEE Journal on Sel. Areas Commun., vol. 31, no. 2, pp. 264-273, Feb. 2013.

[179] H. Huh, G. Caire, H. Papadopoulos, and S. Ramprashad, "Achieving "massive MIMO" spectral efficiency with a not-so-large-number of antennas," IEEE Trans. Wireless Commun., vol. 11, no. 9, pp. 3226-3239, Sep. 2012.

[180] M. Li, Y. H. Nam, B. Ng, and J. Zhang, "A non-asymptotic throughput for massive MIMO cellular uplink with pilot reuse," in IEEE Int. Conf. on Global Commun. (GLOBECOM), Dec. 2012, pp. 4500-4504.

[181] E. Björnson, E. G. Larsson, and M. Debbah, "Massive MIMO for maximal spectral efficiency: How many users and pilots should be allocated?," IEEE Trans. Wireless Commun., vol. 15, no. 2, pp. 1293-1308, Feb. 2016.

[182] M. Ding and D. Lopez Perez, "Please lower small cell antenna heights in 5G," in IEEE Int. Conf. on Global Commun. (GLOBECOM), Dec. 2016, pp. 1-6.

[183] X. Li, S. Zhou, E. Björnson, and J. Wang, "Capacity analysis for spatially non-wide stationary uplink massive MIMO systems," IEEE Trans. Wireless Commun., vol. 14, no. 12, pp. 7044-7056, Dec. 2015.

[184] T. Van Chien, E. Björnson, and E. G. Larsson, "Multi-cell massive MIMO performance with double scattering channels," in IEEE Int. Workshop on Computer Aided Modeling and Design of Commun. Links and Networks (CAMAD), Oct. 2016, pp. 231-236. 